\title{
The Economics and Utilization of Thorium in Nuclear Reactors
}

\section{Technical Annexes 1 and 2}

\author{
Prepared by \\ Resource Planning Associates, Inc. \\ 44 Brattle Street \\ Cambridge, Massachusetts 02138 \\ Subcontract No. 7233
}

\section{OAK RIDGE NATIONAL LABORATORY} OPERATED BY UNION CARBIDE CORPORATION - FOR THE DEPARTMENT OF ENERGY 


\section{DISCLAIMER}

This report was prepared as an account of work sponsored by an agency of the United States Government. Neither the United States Government nor any agency Thereof, nor any of their employees, makes any warranty, express or implied, or assumes any legal liability or responsibility for the accuracy, completeness, or usefulness of any information, apparatus, product, or process disclosed, or represents that its use would not infringe privately owned rights. Reference herein to any specific commercial product, process, or service by trade name, trademark, manufacturer, or otherwise does not necessarily constitute or imply its endorsement, recommendation, or favoring by the United States Government or any agency thereof. The views and opinions of authors expressed herein do not necessarily state or reflect those of the United States Government or any agency thereof. 


\section{DISCLAIMER}

Portions of this document may be illegible in electronic image products. Images are produced from the best available original document. 


\section{Printed in the United States of America. Available frum National Technical Information Service \\ U.S. Department of Commerce 5285 Port Royal Road, Springfield, Virginia 22161 \\ Price: Printed Copy $\$ 9.00$; Microfiche $\$ 3.00$}

This report was prepared as an account of work sponsored by an agency of the United States Government. Neither the United States Government nor any agency thereof, nor any of their employees, contractors, subcontractors, or their employees, makes any warranty, express or implied, nor assumes any legal liability or responsibility for any third party's use or the results of such use of any information, apparatus, product or process disclosed in this report, nor represents that its use by such third party would not infringe privately owned rights. 
Contract No. W-7405-eng-26

subcontract No. 7233

THE ECONOMICS AND UTILIZATION

OF THORIUM IN NUCLEAR REACTORS

TECHNICAL ANNEXES 1 AND 2

Prepared by

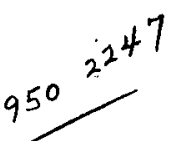

Resource Planning Associates, Inc.

44 Brattle Street

Cambridge, Massachusetts 02138

for

Department of Energy

Washington, D. C. 20545

Date Published; May 1978

This Notice

This report was prepared as an account of work

sponsored by the United States Government. Neither the

United States nor the United States Department of

Energy, nor any of their employees, nor any of their

contractors, subcontractors, or their employees, makes

any warranty, express or implied, or assumes any legal

liability or responsibility for the accuracy, completeness

or usefulutiso of ariy information, apparatus, producr or

process disclosed, or represents that its use would not

infringe privately owned rights.

OAK RIDǴ NATIÓNAL LABORATORY

Oak Rìge, Tennessee 37830

operated by

UNION "CARBIIDE CORPORATION

for the

DEPARTMENT, OF ENERGY 


\section{THIS PAGE}

\section{WAS INTENTIONALLY LEFT BLANK}


CONTENTS

$\underline{\text { Page }}$

ANNEX 1. THORIUM ASSESSMENT PROGRAM SYSTEMS STUDIES

A. INTRODUCTION $\ldots \ldots \ldots \ldots \ldots \ldots \ldots \ldots \ldots \ldots \ldots \ldots \ldots \ldots \ldots \ldots \ldots \ldots \ldots$

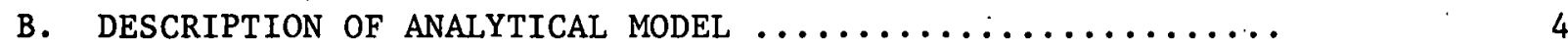

C. DESCRIPTION OF REACTOR DATA ................... 8

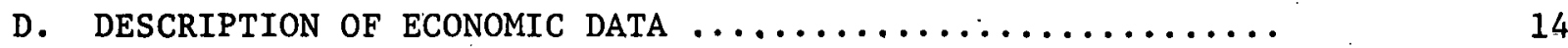

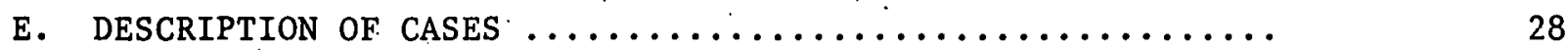

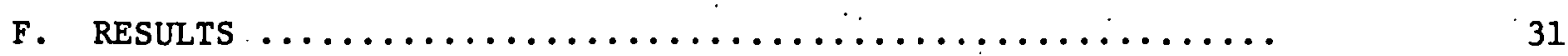

$\begin{aligned} \text { APPENDIX A. } & \text { MATERIAL FLOWS AND NUCLEAR POWER GROWTH } \ldots \ldots \ldots \ldots \ldots \ldots \ldots \ldots \ldots \ldots \ldots \ldots \ldots \ldots \ldots \ldots\end{aligned}$

ANNEX 2. TECHNICAL ASPECTS OF THORIUM UTILIZATION

2.1. INTRODUCTION .......................... 113

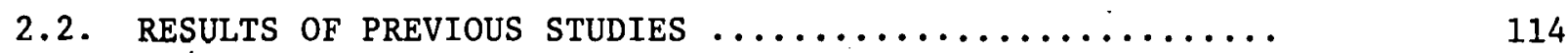

2.3. THE NEED FOR A REVISED ASSESSMENT OF THE THORIUM

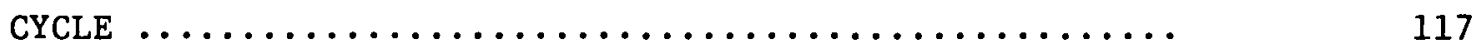

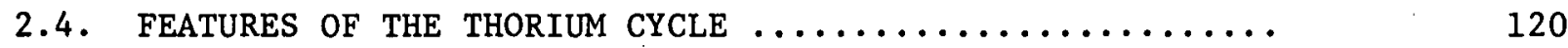

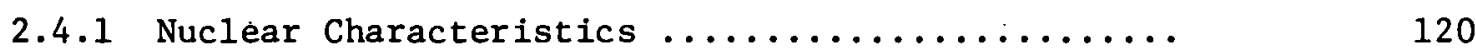

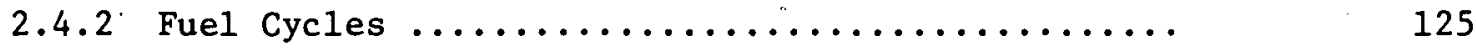

2.4.3 Non-Proliferation Considerations .............

2.4.4 Thorium Supply and Demand ................ 133

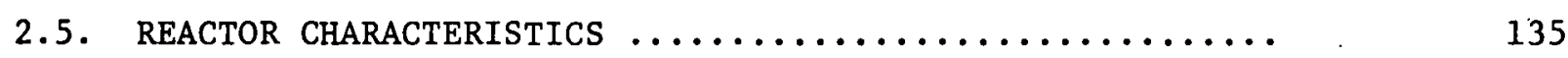

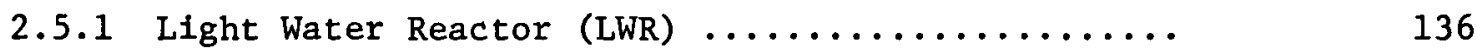

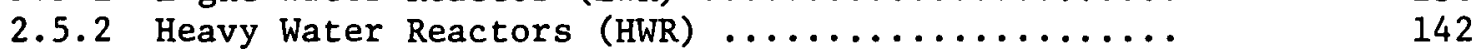

2.5.3 High Temperature Gas-Cooled Reactor (HTGR) ....... 144

2.5.4 Liquid Metal Fast Breeder Reactor (LMFBR) ........ 146

2.5.5 Gas-Cooled Breeder Reactor (GCFR) ............ 148

2.6. FUEL UTILIZATION $\ldots \ldots \ldots \ldots \ldots \ldots \ldots \ldots \ldots \ldots \ldots \ldots \ldots \ldots \ldots \ldots$

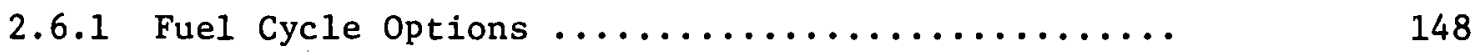

2.6.2 Fuel Cycle Characteristics ................ . 151

2.6.3 High-Temperature Gas-Cooled Reactor ........... 161

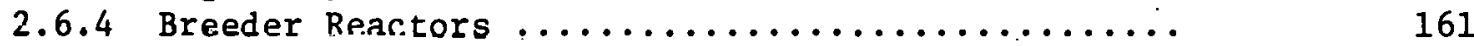

2.6.5 Fuel Cycle Characteristics Utilized in
Thorium Assessment Program .................. 164

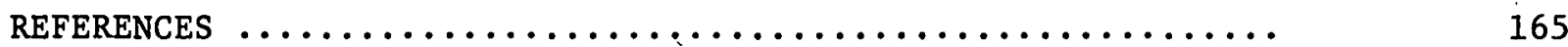




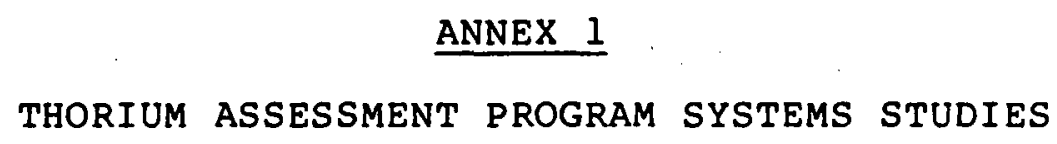

D. R. Haffner, W. E. Black, W. G. Jolly, T. M. Helm, R. W. Hardie, and R. P. Omberg

February 27,1978

HANFORD ENGINEERING DEVELOPMENT LABORATORY 
THIS PAGE

\section{WAS INTENTIONALLY LEFT BLANK}


THORILM ASSESSMENT PROGRAM SYSTEMS STUDIES

D. R. Haffner, W. E. Black, W. G. Jolly, T. M. Helm, R. W. Hardie, and R. P. Omberg

\section{A. INTRODUCTION}

An assessment of the impact of utilizing the U-233/thorium fuel cycle in the U.S. nuclear economy is strongly dependent upon several decisions involving nuclear energy policy. These decisions include: (1) to recycle or not recycle fissile material; (2) if fissile material is recycled, to recycle plutonium, U-233, or both; and (3) to deploy or not to deploy advanced reactor designs such as Fast Breeder Reactors (FBR's), High Temperature Gas Reactors (HTGR's), and Canadian Deuterium Uraniun Reactors (CANDU's), This report examines the role of thorium in the context of the above policy decisions while focusing special attention on economics and resource utilization.

The analysis was performed at the Hanford Engineering Development Laboratory (HEDL) using the ALPS mathematical model of the electrical energy system. The scenarios selected for investigation were developed in a series of meetings attended by Combustion Engineering (CE), Resource Planning Associates (RPA), Oak Ridge National Laboratory (ORNL), Nuclear Research and Applications (NRA/ERDA), and HEDL. The input data, including both reactor design characteristics and nuclear energy system economic parameters, were developed as a result of a cooperative effort by QRNL, United Engineers and Constructors (UE \& C), Argonne National Laboratory (ANL), General Atomic (GA), Uranium Resources and Evaluation (URE/ERDA), CE, RPA, NRA/ERDA and HEDL. 
B. DESCRIPTION OF ANALYTICAL MODEL

The basic components of the ALPS mathematical model (1)' of the electrical energy system are shown in Figure B.1. The model is based on the linear programming technique and evaluates the effect of nuclear policy decisions by computing nuclear and fossil power growth patterns. Given a nuclear energy policy, the model determines the nuclear and fossil power growth pattem which minimizes the total cost of generating electricity over the planning horiznn. In addition to minimizing the electrical generation cost, the following set of constraints are also satisfied.

\section{Total Energy Demand}

The total number of nuclear plus fossil plants built must be sufficient to meet the total energy demand.

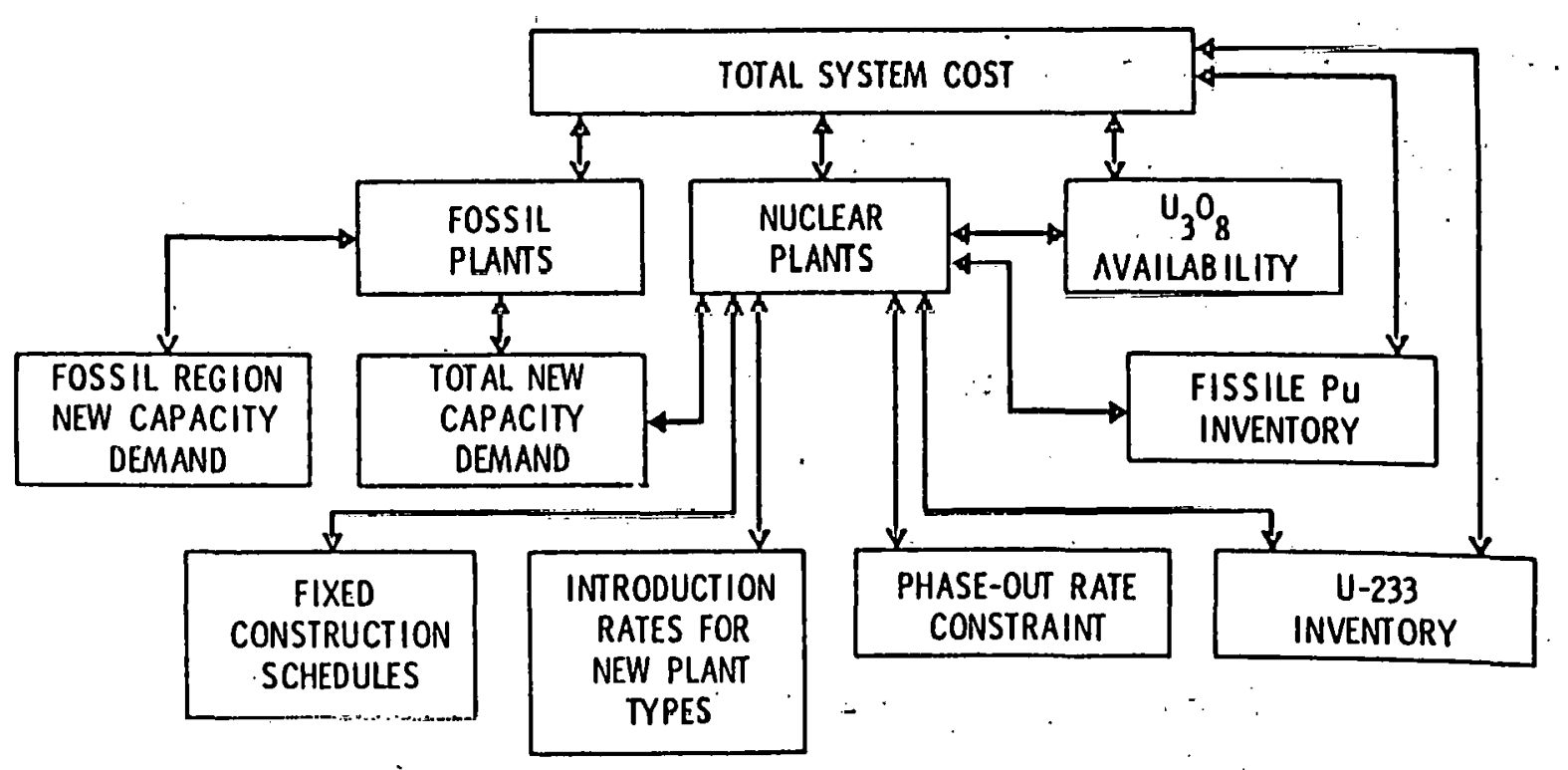

HEDL 7703-067

FICARE B.1: TIL: N.PS ELLLCIRICAL POWER ECONON SUTPLY MODEL 
Reaional Energy Demand and Eossil Fuel Costs

The U.S. is divided into nine regions, each with a unique fossil fuel cost. Nuclear units are thereby forced to compete directly . . . . against localized fossil energy sources.

Uranizon Ore Supply

The long-run marginal cost of urarium ore is computed as a piecewiseconstant function of the cumulative amount of ore mined. This accounts for the increasing cost of ore as the lower grade ore is consumed.

Fissile Euel Supply

A nuclear unit cannot be built unless an adequate supply of fissile fuel (fissile Pu and U233) is available throughout the lifetime of the unit.

\section{Introduction Constraint for a New Technoloay}

A nuclear plant design which differs from established technology can only be introduced at a limited rate. A typical maximam introduction rate is 1 plant during thie first biennium, 2 plants during the second biennium, 4 during the third, 8 during the fourth, etc:

Market Loss Constraint for on Established Technology

If the manufacturing capability to produce a particular reactor type has been established, the rate at which this reactor type will lose its share of the market is limited to a specified percent/year. A typical maximm phase-out rate is $10 \% /$ year. This reflects the fact that some utilities will. continue to purchase plants of an established technology, even though a new technology may be more economical,

A schematic diagram of the nuclear fuel cycle model contained in ALPS is shown in Figure B.2. Charge and discharge data are required for 


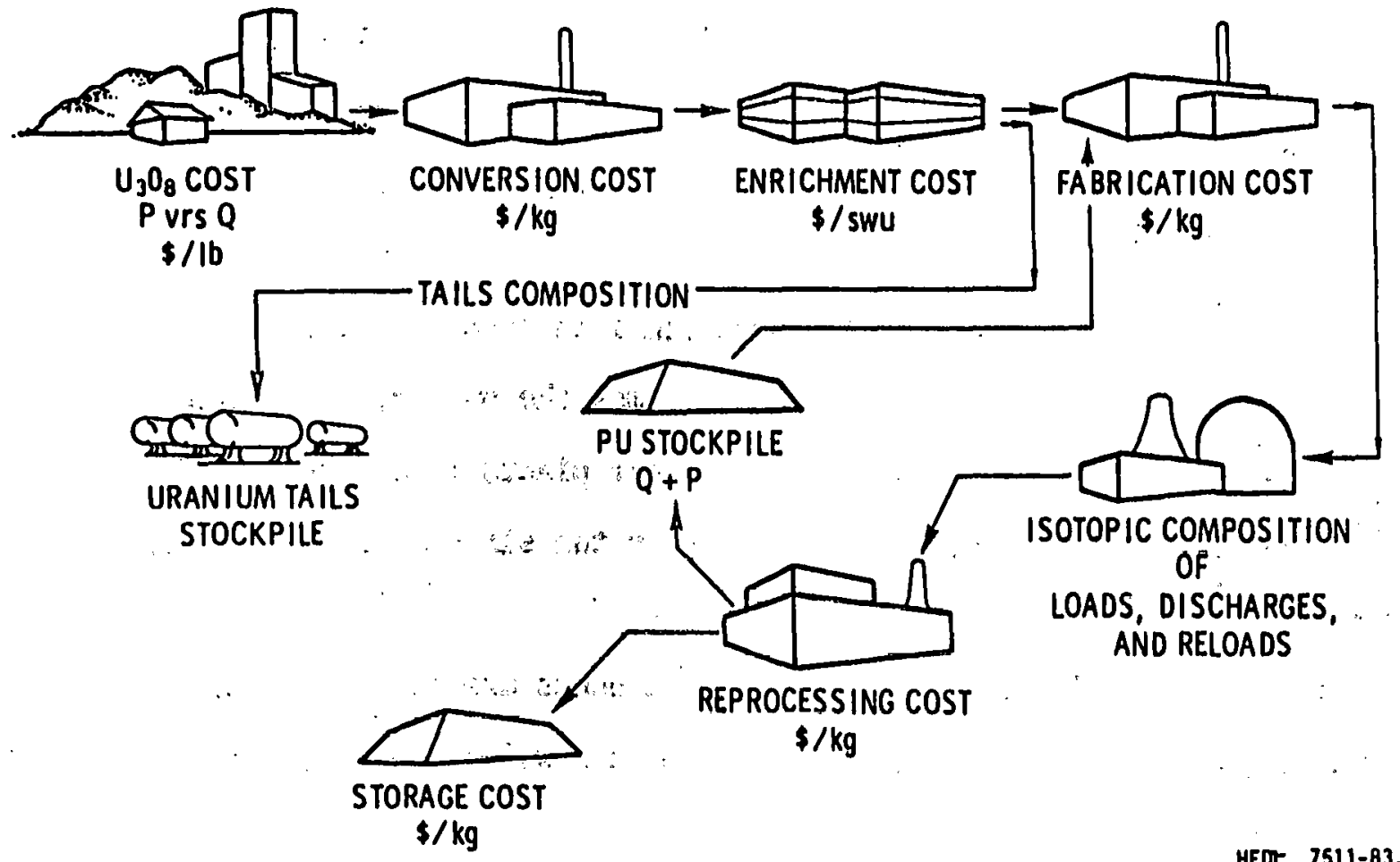

FIGARE В.2: NUCLLAR FULL CYCLE ADDCL

each reactor in each year of operation for the following six nuclear fuel items :

- Th232

- U233

-U235

•U238

-Fissile Pu

-Total Heavy Metal.

The demand for uranium ore $\left(\mathrm{U}_{3} \mathrm{O}_{8}\right)$ and separative work (SWU) is computed from the U-235 and U-238 charge information. Likewise, the $U_{3} \mathrm{O}_{8}$ and SWU spent fuel credits are computed from the discharge information. Fabrication and reprocesssing costs are calculated utilizing the total heavy metal entries 
and the unit fabrication and reprocessing costs in the input data. U-233 and fissile plutonium charge requirements deplete the $U-23 j$ and fissile plutonium stockpiles, while U-233 and fissile plutonium discharges increase the stockpiles.

For fossil plants, the fuel cycle model consists simply of the product of the amount of coal purchased per year times the unit price of coal. The capacity factor of the fossil and nuclear plants is assumed to vary over the lifetime of the plants, and consequently the six nuclear fuel cycle items and the coal usage per year vill also vary.

The total levelized power cost and its components are calculated by ALPS for each power plant available.' The components which are calculated are:

Capital

The capital cost component includes both depreciating and non-depreciating capital costs and also takes salvage value, if any, into account.

\section{Fixed Charaes}

Capital replacements, property insurance, and property taxes are contained in the fixed charge component.

\section{Operation and Maintenance}

The operation and maintenance component includes both fixed and variable $O$ \& $M$ charges, as well as nuclear liability insurance for nuclear plants.

Gross Revenue Tax

The gross revenue tax is simply the gross revenue tax rate times the total revenue.

\section{Income Tas}

The income tax component is obtained by multiplying the income tax rate by the non-deductible expenses. 


\section{Euel}

For nuclear plants, the fuel costs are further divided into $\mathrm{ThO}_{2}, \mathrm{U}_{3} \mathrm{O}_{8}$, U-233, fissile plutonium, enrichment, fabrication, and reprocessing components. The fuel cost for fossil units is limited to the cost of purchasing coal.

\section{DESCRIPTION OF REACTOR DATA}

The reactor characteristics for this study were supplied by $C E$, GA, ANL, and HEDL. Four general types of reactors were included-LWR's, represented by Pressurized Water Reactors (PWR's); HWR's, represented by Canadian Deuterium Uranium Reactors (CANDU:'s); High Temperature Gas Reactors (HTGR's); and Fast Breeder Reactors (FBR's). CE and HEDL provided the data for the PWR's. ANL supplied the data for the CANDU's, GA supplied the data for the HTGR's, and HEDL supplied the data for the FBR's. A list of the reactors included in this study, the power level, and the organization providing the reactor data is shown in Table C.I. Reference 2 contains detailed design data for most of the reactors in this study. The report also includes fuel inventory data used by the ALPS model.

The PWR designs included low-enriched and denatured U-235 fueled reactors, denatured U-233 fueled reactors, and Pu fueled reactors. The diluent consisted of either U-238 or Th, or both for the denatured designs.

In addition to the above PWR designs, three spectral-shift control PWR designs (SSCR's) were included. These were a low-enriched U-235 fueled reactor, a denatured U-233 fueled reactor, and a Pu/Th fueled reactor. 
Table C.1

DESCRIPTION OF REACTORS IN TIIORIIN ASSESSMENT PROGRAM

\begin{tabular}{|c|c|c|c|c|c|}
\hline Name & $\begin{array}{l}\text { Power Level } \\
\text { (Mwe) }\end{array}$ & $\begin{array}{c}\text { Int roduct ion } \\
\text { Date }\end{array}$ & RDC* Labe1 & Fuel Cycle Description & $\begin{array}{l}\text { ganization } \\
\text { Providing } \\
\text { Data }\end{array}$ \\
\hline LWR-US (LE)/U & 1150 & 1969 & PWR-U5 (LE)/U & Low-enriched U-235/U-238 fuel & HEDL \\
\hline -U5 (LE)/U-T & 1150 & 1981 & PWR-U5(LE)/U-T & $\begin{array}{l}\text { Low-enriched U-235/U-238 fuel; } \\
\text { optimized for throwaway }\end{array}$ & IEDL \\
\hline -US (DE)/U/Th & 1150 & 1987 & PHR-U5 (DE)/U/Th-20\% & $\begin{array}{l}\text { Denatured } U-235 \text { fuel }(20 \%) \text { in } \\
\text { U-238 with thorium feed }\end{array}$ & HEDL \\
\hline$-U 3(\mathrm{DE}) / \mathrm{U} / \mathrm{Th}$ & 1150 & 1991 & PWR-U3(DE)/N/Th-12\% & $\begin{array}{l}\text { Denatured.U-233 fuel }(12 \%) \text { in } \\
U-238 \text { with thoriun feed }\end{array}$ & IIEDL \\
\hline$\therefore$ Pu/U & 1150 & 1991 & PWR-Pu/U & $\begin{array}{l}\text { Recycled plutonius fuel in } \\
\text { natural uranitim diluent }\end{array}$ & IIEDL \\
\hline -Pu/'Ih & 1300 & 1991 & PIVR-Pu/Th & $\begin{array}{l}\text { Recycled plutonium fuel in } \\
\text { thorium diluent }\end{array}$ & $\mathrm{CE}$ \\
\hline $\operatorname{SSCR}-115(\mathrm{HE}) / \mathrm{U}$ & 1300 & 1991 & PHR-U5 (LF)/U-SS & $\begin{array}{l}\text { Low-enriched U-235/U-238 fuel } \\
\text { with Spectral Shift Control } \\
\text { Reactor. }\end{array}$ & CE. \\
\hline$-(13(\mathrm{DF}) / \mathrm{J} / \mathrm{Th}$ & 1300 & 1991 & $\begin{array}{l}\text { PWR-U3(DE)/U/Th-SS, } \\
12 \%+\end{array}$ & $\begin{array}{l}\text { Denatured U-233/U-238 (12\%) plus } \\
\text { Mh-232 feed, Spectral Shift Con- } \\
\text { trol Reactor }\end{array}$ & CE \\
\hline$-P u / T h$ & 1300 & 1991 & PIVR-Pu/Th-SS ${ }^{\dagger}$ & $\begin{array}{l}\text { Pu/Thi-232 feed, Spectral Shift } \\
\text { Control Reactor }\end{array}$ & CE \\
\hline HIVR-U5(NAT)/U & 1000 & 1995 & CANDU-US (NAT)/U & Natural uraniun fuel & ANL \\
\hline$-U 5(S B: U) / U$ & 1000 & 1995 & CANDU-U5 (SE)/U & $\begin{array}{l}\text { Slightly enriched uranium fuel } \\
(-1 \% \mathrm{U}-235)\end{array}$ & ANL \\
\hline$-\mathrm{U} 5(\mathrm{Dl}:) / \mathrm{U} / \mathrm{Th}$ & 1000 & 1995 & CANDU-US (DE)/U/Th-20\% & $\begin{array}{l}\text { Denatured U-235/U-238 fuel }(20 \%) \\
\text { plus thorium }\end{array}$ & ANL \\
\hline$-1 J 3(D E) / U / 71 b$ & 1000 & $1995^{\circ}$ & CANDU-U3(DE):/U/Th-128 & $\begin{array}{l}\text { Denatured } U-233 \text { fuel }(128) \text { in } \\
U-238 \text { plus thoriun with } U-233 \\
\text { self-generated recycle }\end{array}$ & ANL \\
\hline$-P M / I I$ & 1000 & 1995 & $\mathrm{CANDU}-\mathrm{Pu} / \mathrm{U}$ & Plutonium fuel in natural uranium & ANL \\
\hline$\therefore \mathrm{Pu} / \mathrm{in}$ & 1000 & 1995 & CANDJ-Pu/Th & Plutonium fuel in thorium & ANL \\
\hline
\end{tabular}


Table C.1

DESCRIYYION OI: RI:ACTORS IN TIORILM ASSESSMENT PROCRAM

(CONT'D.)

\begin{tabular}{|c|c|c|c|}
\hline Name & $\begin{array}{c}\begin{array}{c}\text { Power Level } \\
\text { (Anlei }\end{array} \\
\end{array}$ & $\begin{array}{c}\text { Introduction } \\
\text { Date }\end{array}$ & RDC* Label \\
\hline ITTGR-US(LE)/U-T & 1344 & 1995 & HTGR-US(LE)/U-T \\
\hline -US (IE)/U & 1344 & 1995 & HTGR-U5 (LE)/U \\
\hline -U5 (DГ:)/U/רh & 1344 & 1995 & IITGR-U5(DE)/U/Th-208 \\
\hline$-\mathrm{US}(\mathrm{HIE}) / \mathrm{Th}$ & 1344 & 1995 & ITTrR-US (IE)/Th \\
\hline$-U 3(I D E) / U / T h$ & 1344 & 1995 & ITTGR-U3(DE)/N/Th-158 \\
\hline- -U3/Th & 1344 & 1995 & HTGR-U3/Th \\
\hline -Purth & 1344 & 1995: & HTGR-Pu/Th \\
\hline FBR-Pu-U/U & 1200 & 2001 & LMFBR-Pu/U/U/U-LT \\
\hline . & & & \\
\hline$-\mid M u-I I / T h$. & 1200 & 2001 & LAFBR-Pu/U/Th/Th-LT \\
\hline
\end{tabular}

Fuel Cycle Description

Low-enriched U-235/U-238 fuel; optimized for throwaway

Low-enriched U-235/U-238 fuel; optimized for recycle

Denatured U-235 fuel (208) in U-238 plus thorium; optimized for recycle

lligh-enriched U-235 fuel plus thoriun

Denatured U-233 fuel (15\%): in U-238 plus thorium; optimized for recycle

U-233 and thorium fuel with U-233 self-generated recycle

Plutonium plus thorium fuel

Pu/U-238 core feed and U-238 blanket feed; low temperature design, oxide fuel

$\mathrm{Pu} / \mathrm{U}-238$ core feed and Th-232. blanket feed; low temperature design, oxide fuel
Organization

Providing Data

GA

GA

GA

GA

GA

GA

IEDL

HIEDL.

*Reactor design documented in "Reactor Design Characteristics and Fuel Inventory Data", IEDL-TC-971, (Oct. 1977)

${ }^{\dagger}$ Reactor data not received in time for inclusion in original RDC document 
The CANDU designs were represented by three U-235 fueled reactors (natural, slightly-enriched, and denatured), a denatured U-233 fueled reactor, a Pu/U-238 reactor, and a $\mathrm{Pu} / \mathrm{Th}$ reactor.

HTGR designs consisted of low-enriched, denatured, and high-enriched U-235 fueled reactors, denatured and high-enriched U-233 fueled reactors, and a Pu/Th reactor.

The FBR designs included two plutonium fueled versions; one having a uranium blanket and the other a thorium blanket.

Introduction dates for each reactor type are also given in Table C.I. A slight modification to an existing PWR fuel design, such as a thicker fuel pin cladding to increase discharge exposure, was introduced in 1981. A more extensive modification; such as a denatured U-235 PWR fuel pin, was delayed until 1987. The remaining PWR designs, including the spectral-shifts, were introduced in 1991. The HWR's and HTGR's were all introduced in 1995 while the FBR's were not introduced until 2001:

Finally, Table C.2 presents the key nuclear characteristics for each reactor design included in the study. In particular, lifetime $\mathrm{U}_{3} \mathrm{O}_{8}$ and separative work requirements and equilibrium fabrication requirements, discharge exposures, conversion ratios, fissile plutonium flows, and fissile U-233. flows are given. The table shows that, for the throwaway cycle, low-enriched HTGR's offer significant (almost 20\%) uranium ore savings compared to low-enriched PWR's. . Similarly, slightly-enriched CANDU's reduce uranium ore requirements by an additional $25 \%$ over HTGR's. and more than $35 \%$ over PWR's. Although low-enrlched PWR's and HITR's, have roughly the same enrict. Ant requirements, the slightly enriched CANDU's require 5-6 times less enrichment. Core discharge exposures for 
Table C. 2

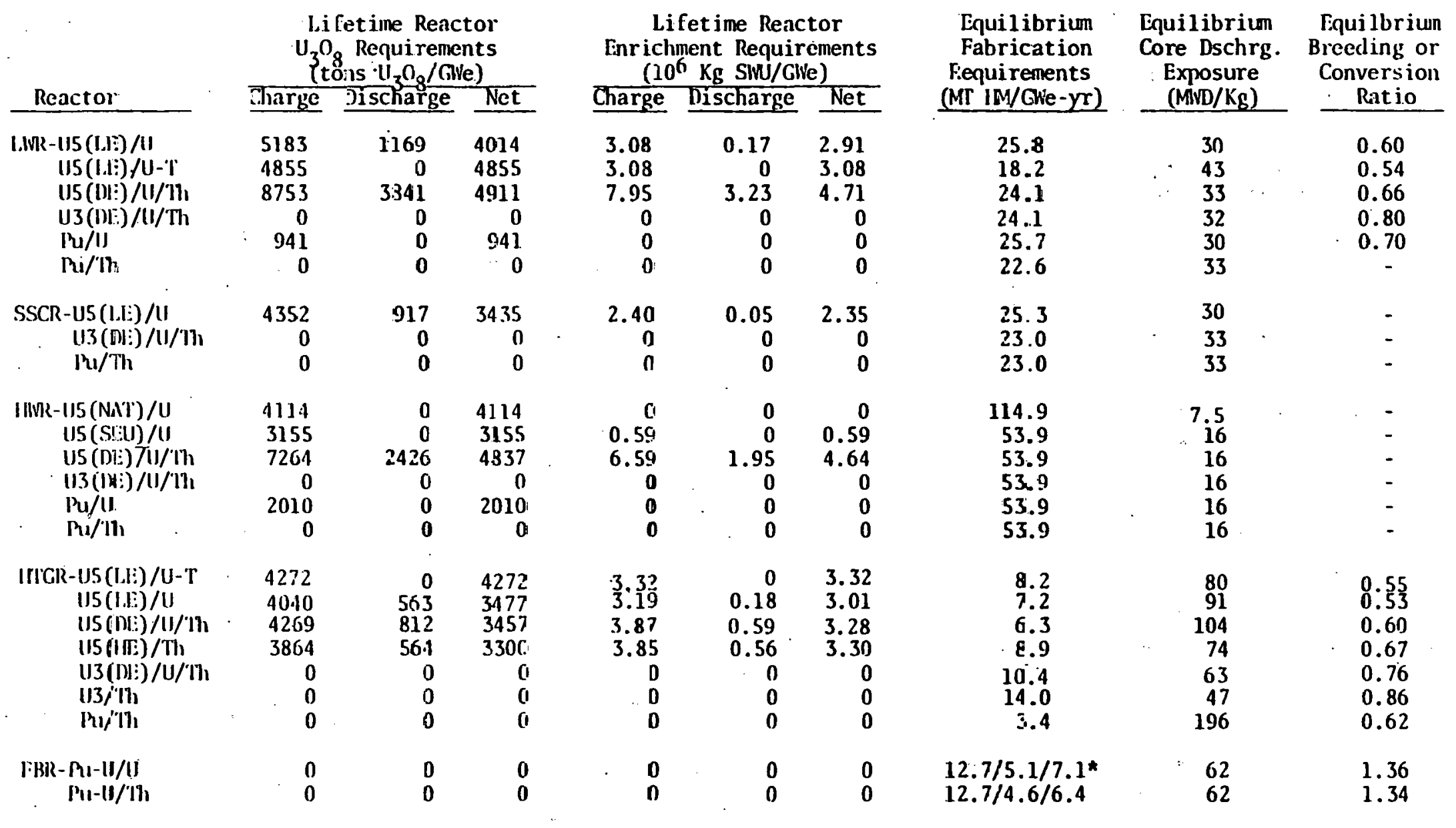

*Core/Rad. Bl kt./Ax. B1kt. 
Table C. 2

(CONT' 1 )

REACTOR CIURACTI:RISTICS

\begin{tabular}{|c|c|c|c|}
\hline \multirow[b]{2}{*}{ Reactor } & \multicolumn{3}{|c|}{$\begin{array}{c}\text { Average* Pissile Plutonitum } \\
\text { Mass Flows } \\
(\text { Kg/GWe-yr })\end{array}$} \\
\hline & đlarge & Discharge & Net \\
\hline $\operatorname{l.MR}-1 / 5(\mathrm{I} . \mathrm{I}) / \mathrm{U}$ & $\mathbf{0}$ & 146.8 & 146.8 \\
\hline U5(LE) $/ U-\mathrm{T}$ & $\mathbf{0}$ & 0 & 0 \\
\hline US (I)I:)/UV/Th & 0 & 77.8 & 77.8 \\
\hline U3(b[) / / J/Ih & 0 & 88.2 & 88.2 \\
\hline $\mathrm{Pu} / \mathrm{u}$ & 700.6 & 472.2 & -228.4 \\
\hline $\mathrm{Mi} / \mathrm{Th}$ & 1294.1 & 620.2 & -67.3 .9 \\
\hline SSCR:-U5 (I.H)/L & 0 & .185 .0 & 185.0 \\
\hline $\mathrm{H} 3(\mathrm{Dl}:) / \mathrm{U} / \mathrm{Ih}$ & 0 & 72.9 & 72.9 \\
\hline $\mathrm{Tu} / \mathrm{Th}$ & 1202.3 & 556.4 & -645.9 \\
\hline InVRL-U5 (NAT)/U & 0 & 290.4 & 290.4 \\
\hline US (SLU) $/ \mathrm{II}$ & 0 & $159: 8$ & 159.8 \\
\hline U5(Dl:)/U/Th & 0 & 22.5 & 22.5 \\
\hline U3(I): $) /(1 / T h$ & 0 & 26.9 & 26.9 \\
\hline$P u / U$ & 156.6 & 177.7 & 21.1 \\
\hline Pu/Th & 895.5 & 234.4 & -661.1 \\
\hline $1516 \pi-115(1.18) / 11-1$ & 0 & 0 & 0 \\
\hline U5(1.1:)/11 & 0 & 52.4 & 52.4 \\
\hline $115(111:) / 1 / / T h$ & 0 & 31.2 & 31.2 \\
\hline $105(111) / 1 \%$ & 0 & 1.0 & 1.0 \\
\hline $113(111:) / 11 / 1 \mathrm{Th}$ & 0 & 33.1 & 33.1 \\
\hline $113 / 1 \mathrm{lh}$ & 0 & $\mathbf{0}$ & 0 \\
\hline P'u/l'th & 637.0 & 126.7 & -510.3 \\
\hline FBBR-PU-IU/U & 1253 & 1526 & 273.3 \\
\hline$|u-1| / 2 h_{1}$ & 1261 & 1283 & 21.9 \\
\hline
\end{tabular}

\begin{tabular}{|c|c|c|}
\hline & $\begin{array}{c}\text { Average* U-233 } \\
\text { Mass Flows } \\
\text { (Kg/Gie-yr) }\end{array}$ & \\
\hline Charge & Discharge & Net \\
\hline 0 & 0 & 0 \\
\hline 0 & 0 & 0 \\
\hline 0 & 256.2 & 256.2 \\
\hline 807.0 & 530.4 & -276.6 \\
\hline 0 & 0 & 0 \\
\hline 0 & 239.0 & 239.0 \\
\hline 0 & $\mathbf{0}$ & 0 \\
\hline 619.9 & 426.2 & -193.7 \\
\hline 0 & 281.2 & 281.2 \\
\hline 0 & 0 & 0 \\
\hline 0. & 0 & 0 \\
\hline 0 & 418.2 & 418.2 \\
\hline 765.8 & 664.7 & -101.1 \\
\hline 0 & 0 & 0 \\
\hline 0 & 391.9 & 391.9 \\
\hline 0 & 0 & 0 \\
\hline 0 & 0 & 0 \\
\hline 0 & 104.6 & 104.6 \\
\hline 0 & 186.9 & 186.9 \\
\hline 489.5 & 264.9 & -224.6 \\
\hline 501.5 & 389.0 & -112.5 \\
\hline 0 & 94.1 & 94.1 \\
\hline $\begin{array}{l}0 \\
0\end{array}$ & $\begin{array}{r}0 \\
237.5\end{array}$ & $\begin{array}{r}0 \\
237.5\end{array}$ \\
\hline & & \\
\hline
\end{tabular}

*l.i fetinic averige with $1 \%$ fabrication ind $1 \%$ reprocessing losses. 
FBR's are approximately twice the exposures of PWR's while CANDU's exposures are about half the PWR's exposures. HTGR discharge exposures are extremely large--nearly $200 \mathrm{MWd} / \mathrm{Kg}$ for the Pu/Th fuel design.

\section{DESCRIPTION OF ECONOMIC DATA}

The economic data base used in the thorium assessment program was jointly developed by CE, ORNL, UE \& C, ANL, RPA, HEDL, URE/ERDA, and NRA/ERDA. The data base includes capital costs, operation and main: tenance costs, fabrication and reprocessing costs, capacity factors, money costs, estinates of projected electrical generation, regional coal data, and construction cash flow data.

The deflated and present-valued capital costs excluding interest during construction for LWR's, SSCR's; HTGR's, CANDU's, and FBR's are shown in Table D.1. The same capital costs including interest during construction are shown in Table D.2. In either case, the stream of

Table D.1

CAPITAL COSTS

$(7 / 1 / 76 \$ /$ kive not including IDC)

Power P1ant Type

LWR

LWR-spectral shift

HTGR

CANDU

FBR

$\frac{\text { Capital Costs }}{500(1)}$
$520+38$ (heavy water) $=558^{(2)}$
560 to $580^{(1)}$
$605+156$ (heavy water) $=761^{(1)}$
625 to $650^{(1)}$

(1) Supplied by United Engineers and Constructors

(2) Supplied by Combustion Engineering 
Table D.2

CAPITAL COSTS

$(1 / 1 / 77 \$ / \mathrm{kWe}$ including IDC)

Power Plant Type

LWR

LWR-spectral shift

HTGR

CANDU

FBR

Coal

with scrubber

without scrubber
Capital Cost

625

$650+40$ (heavy water) $=690$

715

$755+160$ (heavy water) $=915$

800

550

475

expenses incurred during the construction of the plant is discounted to the date of start-up and is measured in dollars of constant purchasing power. The capital cost of the SSCR--including the initial heavy water inventory--was assumed to be $10 \%$ greater than that of the standard LiWR. The capital cost of the dry CANDU--i.e., excluding the initial heavy water inventory--was assumed to be $21 \%$ greater than that of the standard LWR; and the capital cost of the wet CANDU was assumed to be $46 \%$ greater than that of the standard IWR. The capital cost of the FBR was assumed to be $25 \%$ greater than that of the standard LWR. . The capital cost of a coal unit without scrubbers was assumed to be $24 \%$ less than that of the standard LWR, while the capital cost of a coal unit with scrubbers was assuned to be $12 \%$ less than that of the standard LiNR. The cost of scrubbers was assumed to be $75 \$ /$ kwe and $5 \%$ of the power generated by the plant was assumed to be consumed in their operation. 
The operation and maintenance costs for the same power plants are shown in Table D.3. The operation and maintenance costs for the SSCR and the CANDU are higher than the standard LWR because of the heavy water replacement requirement and because of the necessity to perform some maintenance in atmospheres containing tritium.

The fabrication costs for the PWR, SSCR, HTGR, CANDU, and FBR are shown in Table D.4. The fabrication costs for the PWR and the SSCR were based on the standard $17 \times 17$ pin assembly with a fuel pin of $374 \mathrm{mils}$ in outer diameter. The fabrication costs for the HTGR were based on the standard carbon-coated uranium carbide fissile microsphere subsequently formed into cylindrical rods and located in a hexagonal graphite block. The fabrication costs for the CANDU were based on a 37 pin assembly approximately 20 inches long with a fuel pin of 531 mils in outer diameter. The fabrication costs for the FBR were based on a 217 pin assembly in a

Table D.3

OPERATION AND MAINTENANCE COSTS

$(1 / 1 / 77 \$ /$ kWe-year $)$

\begin{tabular}{lcc} 
Power Plant -Type & Fixed & Variable* \\
\cline { 2 - 2 } & 3.6 & 1.9 \\
HTGR & 3.6 & 1.9 \\
CANDU & 7.2 & 3.8 \\
FBR & 4.1 & 2.3 \\
& & \\
Coal & & \\
with scrubber & 4.2 & 11.7 \\
without scrubber & 4.0 & 4.8 \\
\hline
\end{tabular}

* Must be multiplied by the capacity. factor for given year 
Table D. 4

Fabrication Costs

(1/1/77 \$/kg HM)

Reactor Type

Cost (year) Schedule

PIV

LE U5-U8

DE US-U8-Th

Pu-U

Pu-Th

DE U3-U8-Th

$\operatorname{SSCR}$

LE U5-U8

DE US-UB-Th

PL:-U

$\mathrm{Pr}-\mathrm{Th}$

DE U3-U8-Th

HTGR

HE US-U8-Th

$\mathrm{C} / \mathrm{Th}+\mathrm{U}=150$

$\mathrm{C} / \mathrm{Th}+\mathrm{U}=238$

$C / T h+U=335$

$\mathrm{C} / \mathrm{Th}+\mathrm{U}=400$

$\mathrm{C} / \mathrm{TT}_{2}+\mathrm{U}=650$

DE US-U8-Th

$\mathrm{C} / \mathrm{Th}+\mathrm{U}=150$

$\mathrm{C} / \mathrm{Th}+\mathrm{U}=238$

$\mathrm{C} / \mathrm{Th}+\mathrm{U}=335$

$C / T h+U=400$

$\mathrm{C} / \mathrm{Th}+\mathrm{U}=650$

HE U3-U8-Th

$\mathrm{C} / \mathrm{Th}+\mathrm{U}=150$

$C / T h+U=238$

$\mathrm{C} / \mathrm{Th}+\mathrm{U}=335$

$C / T h+U=400$

$C / T h+U=650$

DE U3-U8-Th

$\mathrm{C} / \mathrm{Th}+U=150$

$\mathrm{C} / \mathrm{Th}+U=238$

$\mathrm{C} / \mathrm{Th}+\mathrm{U}=335$

$\mathrm{C} / \mathrm{Th}+\mathrm{U}=400$

$\mathrm{C} / \mathrm{th}+\mathrm{U}=650$

$\mathrm{Pu}-\mathrm{Th}$

$\mathrm{C} /$ Th $=238$

CANDU

NAT U5-U8

SE U5-U8

HE US-U8-Th

DE US-U8-Th

Pu-U

Pu-Th

DE U3-U8-Th

FBR

Pu-U8 core (oxide)

Pu-Th core (oxide)

U3-U8 core (oxide)

U8 axial blanket (oxide)

Us radial blanket (oxide)

Th axial blanket (oxide)

Th radial blanker (oxtde)

$100(1969+2089)$

$230(1987)+140(1997)$

$550(1991)+340(2001)$

$550(1991)+340(2001)$

$880(1991)+550(2001)$

$100(1991 \rightarrow 2089)$

230 (1991) - 140 (2001)

$550(1991) \rightarrow 340^{\circ}(2001)$

$550(1991)-340(2001)$

$880(1991)+550(2001)$

$$
\begin{aligned}
340(1995) & +210(2005) \\
500(1995) & +300(2005) \\
660(1995) & +400(2005) \\
760(1995) & +470(2005) \\
1220(1995) & +770(2005) \\
& \\
340(1995) & +210(2005) \\
500(1995) & +300(2005) \\
660(1995) & +400(2005) \\
760(1995) & +470(2005) \\
1220(1995) & +770(2005)
\end{aligned}
$$

860 (1995) + $470(2005)$

$1220(1995)+670$ (2055)

1640 (1995) - 900 (2005)

2000 (1995) - $1100(2005)$

$3200(1995)+1750(2005)$

$860(1995) \rightarrow 470(2005)$
$1220(1995)+670(2005)$
$1640(1995) \rightarrow 900(2005)$
$2000(1995)=1100(2005)$
$3200(1995)=1750(2005)$

$1220(1995)+670(2005)$

$60(1995+2089)$

$60(1995+2089)$

140 (1995) - 85 (2005)

$140(1995)+85(2005)$

$320(1995)+200(2005)$

$320(1995)+200(2005)$

$560(1995)+350(2005)$

$1750(2001)+950(2011)$

$1750(2001)+950(2011)$

$3000(2001)+1650(2011)$

$35(2001)+25(2011)$

$250(2001)+150(2011)$

35: $(2001)+25(2011)$

$250(2001) \cdot 150$ (2011) 
hexagonal duct with a fuel pin of 310 mils in outer diameter. The fabrication costs are shown for both the standard and alternate fuel designs, with a lowenriched fuel denoted by LE, a high-enriched fuel denoted by $\mathrm{HE}$, and a denatured fuel denoted by DE. The costs are shown as a function of time beginning with the expected introduction date for a particular reactor and fuel design. If a particular reactor and fuel design should prove successful, fabrication costs should decrease as larger plants with higher. throughput rates are constructed. The decrease in fabrication costs over the first decade after introduction as shown in Table D.4 is simply indicative of a transition from small fabrication plants with high unit costs to larger fabrication plants with lower unit costs. The fabrication costs in Table D.4 are a strong function of the fissile isotope and a weak function of the fertile isotope. The sensitivity to the fissile isotope is caused by either the spontaneous fission associated with high-exposure fissile plutonium or the gamma activity associated with high-exposure U-233. The costs are based on the assumption that fuels containing U-235 are fabricated on a Line with contact operation and contact maintenance, while fuels containing fissile plutonium are fabricated on a line with remote operation and contact maintenance, and fuels containing U-233 are fabricated on a line with remote operation and remote maintenance.

The expected reprocessing costs are shown in Table D.5. These costs were obtained by estimating the capital and operating costs associated with each of five stages of the reprocessing process. The stages were: headend, solvent extraction, product conversion, offgas treatment, and waste treatment. The costs are shown as a function of time reflecting the transition from a new industry consisting of small plants with high unit costs to a mature industry consisting of 
Table D.5

\section{Reprocessing Costs*}

$(\$ / \mathrm{kg} \mathrm{HM})$

Reactor Type
LWR
SSCR
HTGR
CANDU
FBR

$$
\begin{aligned}
& \text { Cost (year) Schedule } \\
& 225(1991)+150(2001) \\
& 225(1991)+150(2001) \\
& 800(1995)+400(2005) \\
& 225(1995)+150(2005) \\
& 500(2001)+200(2011)
\end{aligned}
$$

*Does not include spent fuel shipping costs, waste shipping costs, or waste storage costs.

larger plants with lower unit costs. The expected cost for spent fuel shipping, waste shipping, and waste storage is shown in Table D.6. The sum of the reprocessing cost, the spent fuel shipping cost, the waste shipping cost, and the waste storage was defined as the total reprocessing cost, and this is shown in Table $D .7$. The cost of throwaway without reprocessing is also shown in Table $D .7$.

The long-run marginal cost of $\mathrm{U}_{3} \mathrm{O}_{8}$ as a function of the cumulative amount mined is shown in Table D.8. Two projections are shown in the table-viz, a small supply and a large supply. The small supply is based on the assumption that 2.3 million tons of $U_{3} O_{8}$ will be available from conventional uraniun ore resources, while the large supply is based on the assumption that 4.2 million tons of $\mathrm{U}_{3} \mathrm{O}_{8}$ will be available from conventional resources. In either case, the shales are assumed to be mined after the conventional resources are depleted. The cost of extracting 
Table D.6

SHIPPING AND WASTE STORAGE COSTS

$(\$ / \mathrm{kg} \mathrm{HM})$

\begin{tabular}{|c|c|c|c|}
\hline Reactor Type & $\begin{array}{c}\text { Spent Fue1 } \\
\text { Shipping Costs* }\end{array}$ & $\begin{array}{l}\text { Waste Shipping } \\
\text { Costs } \\
\end{array}$ & $\begin{array}{c}\text { Waste Storage } \\
\text { Costs } \\
\end{array}$ \\
\hline LWR & 15 & 10 & 40 \\
\hline HTGR & 70 & 10 & 40 \\
\hline CANDU & 15 & $=10$ & 40 \\
\hline FBR & 45 & 10. & 40 \\
\hline
\end{tabular}

*Throwaway includes shipping plus $\$ 100 / \mathrm{kg}$ HM permanent spent fuel storage

Table D.7

TOTAL REPROCESSING COSTS*

$(\$ / \mathrm{kg} \mathrm{HM})$

$\begin{array}{llc}\begin{array}{l}\text { Reactor } \\ \text { Type }\end{array} & \text { Cost (year) Schedule } & \begin{array}{c}\text { Throwaway } \\ \text { Cost Schedule }\end{array} \\ \text { LWR } & 290(2001)+215(2011) & 125 \ldots \\ \text { HTGR }^{\prime} & 920(2001)+520(2011) & 180 \\ \text { CANDU } & 290(2001)+215(2011) & 125 \\ \text { FBR } & 545(2001)+295(2011) & 155\end{array}$

Total reprocessing costs include spent fuel shipping costs, reprocessing costs ( $\$ 100 / \mathrm{kg}$ HM for throwaway cycle), waste shipping costs, and waste storage costs. 
Table D.8

$\mathrm{U}_{3} \mathrm{O}_{8}$ Assumptions

Large $\mathrm{U}_{3}{ }_{-}-$Supply $_{-}$

\begin{tabular}{cc}
$\begin{array}{c}\text { Quantity of } U_{3}{ }^{0} 8 \\
10^{6} \text { tons }\end{array}$ & $\begin{array}{c}\text { Long-Rum } \\
\text { Marginal Cost } \\
\$ / 1 b\end{array}$ \\
\hline $0.0-0.25$ & 14 \\
$0.25-0.75$ & 23 \\
$0.75-1.25$ & 33 \\
$1.25-1.75$ & 44 \\
$1.75-2.5$ & 53 \\
$2.5-3.5$ & 61 \\
$3.5-4.25$ & 80 \\
$4.25-4.75$ & 107 \\
$4.75-5.25$ & 128 \\
$5.25-5.75$ & 143 \\
$5.75-8.5$ & 165 \\
above 8.5 & 180
\end{tabular}

Small U ${ }_{3}{ }^{\circ}$. Supply Long-Run $\begin{array}{r}\begin{array}{r}\text { Ruantity of } \mathrm{U}_{3} \mathrm{O}_{8} \\ 10^{6} \text { tons }\end{array} \\ \hline\end{array}$ Marginal Cost $\$ / 1 b$ 14 24 35

$0.75-1.25$ 54

$1.25-1.75$ 84

$1.75-2.25$ 128

$2.25-2.75$ 158

$2.75-3.25$

$3.25-3.75$ 173

$3.75-4.25 \quad 180$

$4.25-4.75$ 180

$4.75-6.5$ 210 above 6.5

the shales increases from $125 \$ / 1 b$ to $240 \$ / 1 b$ for the small supply case, while the cost of extracting the shales increases from $100 \$ / 1 \mathrm{~b}$ up to 180 $\$ / 1$ b for the large supply case.

The enrichment cost and tails composition assuming a continuation of the gaseous diffusion technology is shown in Table D.9. If the gaseous diffusion technology is continued, it was assumed that the tails composition will be stabilized at 0.0020 and that the cost of 
enrichment will increase to $80 \$ /$ SWJ in 1987 and remain constant thereafter.

Additional data pertaining to fabrication, reprocessing, and minor reactor costs are shown in Table D.10. The first reprocessing

Table D.9

Enrichment Cost and Tails Composition

\begin{tabular}{|c|c|c|c|c|}
\hline Year & & Composition & & Cost \\
\hline 1969 to 1976 & & 0.0020 & & $50 \$ /$ SWU \\
\hline 1977 to 1986 & $\cdots$ & 0.0020 & $\therefore$ & $75 \$ / S W U$ \\
\hline 1987 to 2089. & & 0.0020 & $\therefore$ & $80 \$ / S W U$ \\
\hline
\end{tabular}

Table D.10

Additional Data

Fabrication and Reprocessing.

Reprocessing Introduction Data

1991

Out-of-Reactor Time (fabrication

lead time + reprocessing lag tine)

2 years with reprocessing, and extended to after 1991 prior to reprocessing

Tigsile Storage Charges

After Reprocessing

$2 \$ / y=y r$ for $233_{y}$ and fissile plutonium

Cost of Converting $\mathrm{U}_{3} \mathrm{O}_{8}$

$\$ 3.50 / \mathrm{kg}$ of U

Minor Reactor Costs

Property Insurance Rate $\quad 0.0025$

Capital Replacement Rate 0.0035

Nuclear Liability $580 \times 10^{3} \$ / \mathrm{yr}$ 
plant is assumed to be in operation in 1991--provided a policy decision has been made to reprocess spent fuel. All fuel discharged from the reactor prior to reprocessing is assumed to be stored, and the spent fuel stockpile is reduced in an orderly manner after the advent of reprocessing. The out-of-reactor time required for reprocessing and refabrication is assumed to be two years after the spent fuel stockpile has been recuced to zero.

The capacity factor of a plant as a function of time throughout its thirty year life is shown. in Table D.11. The capacity factor increases from 608 to 728 during the first three years of operation, and remains at 728 during the subsequent fourteen years. The capacity factor then decreases continuously as the forced outage rate increases and as the plant is shifted from a base-load unit to an intermediateload unit.

The long-term real cost of money to the electric utility industry is shown in Table D.12. These costs were developed by analyzing the deflated cost of debt and equity to the industry over the past thirty years. The long-term deflated cost of debt has been $2.5 \% / y r$ and the long-term deflated cost of equity has been $7.0 \% / y r$. Assuming the industry to be funded at approximately $55 \%$ debt and $45 \%$ equity, the long-term real money cost is approximately $4.5 \% / \mathrm{yr}$.

The projected electrical generation from 1975 to 2030 including both nuclear and fossil is shown in Table D.13. The energy requirement is based on the assumption of a growth rate of $5.6 \% / y r$ between 1975 and 1980 continuously decreasing thereafter to $2.5 \% / y$ between 2020 and 2030. This energy projection assumes $5 \%$ of the energy is produced by peaking generation plants. 
Table D.11

Plant Capacity Factors

\begin{tabular}{cc} 
Year & Capacity Factor, 8 \\
\hline 1 & 60.0 \\
2 & 66.0 \\
3 & 72.0 \\
4 & 72.0 \\
. & $\cdot$ \\
. & $\cdot$ \\
. &. \\
15 & 72.0 \\
16 & 72.0 \\
17 & 70.4 \\
18 & 68.9 \\
19 & 67.3 \\
20 & 55.7 \\
21 & 64.1 \\
22 & 62.6 \\
23 & 61.0 \\
24 & 59.4 \\
25 & 57.9 \\
26 & 56.3 \\
27 & 54.7 \\
28 & 53.1 \\
29 & 51.6 \\
30 & 50.0
\end{tabular}

Table D.12

Money Costs

Debt Interest

$2.5 \%$

Equity Interest

$7.0 \%$

Percent Debt

0.55

Percent Equity

0.45

Effective Interest Rate

$4.525 \%$ 
Table D.15

Projected Total Electrical Generation

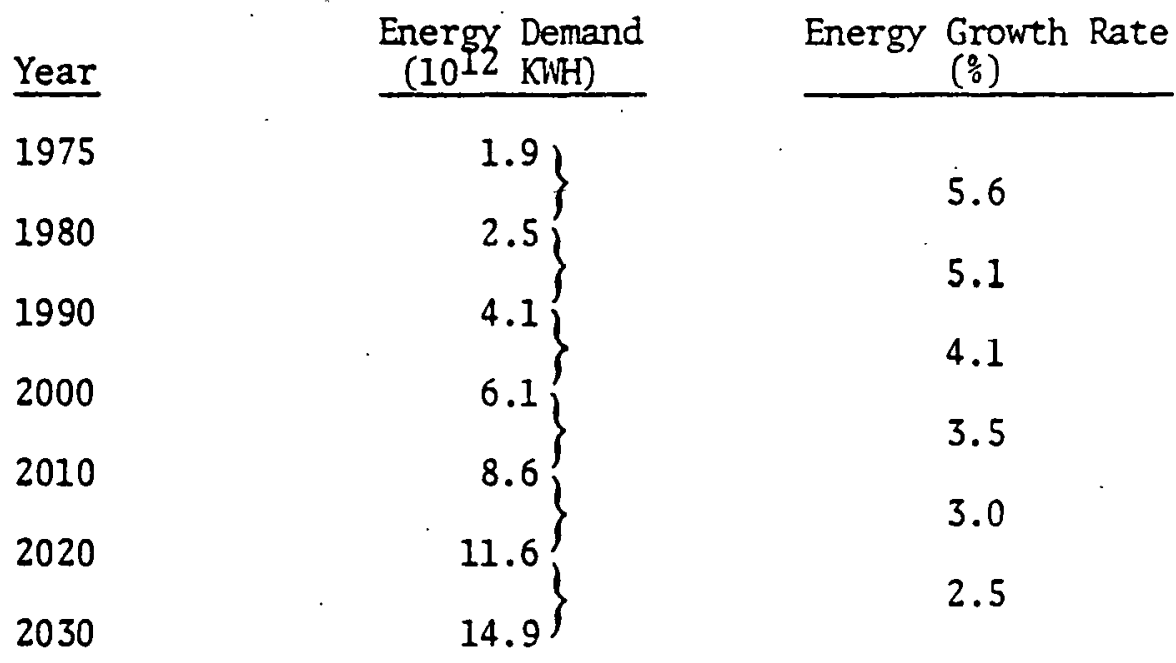

The relative amount of electric energy consumed in the U.S. by geographical region is shown in Table D.14. This information, along with the average cost and heat content of the coal utilized in the same regions, is used to characterize the principal competitor to nuclear energy. The average cost and heat content of the coal utilized by region as a function of time are shown in Table 0.15. The cost of coal ranges from a low value of $6.04 \$ /$ ton at $6,600 \mathrm{Btu} / 1 \mathrm{~b}$ in the West South Central Region to a high value of $33.89 \$ /$ ton at 13,500 Btu/lb in the Northeast Region. The real cost of coal was assumed to be constant as a function of time.

Tables D.16 - D.18 are used for capital requirements analysis. Capital cost cash flows representing the stream of paymențt during the construction period are shown in Table D.16. Capital costs for fabrication, reprocessing and enrichment facilities and for uranium and thorium mines are given in Tables D.17. and D.18. 
Table D.14

Percent of Total U.S. Electrical Sales

by FPC Consuming Region

$\begin{array}{lrr} & \underline{1975} & \frac{1974}{3.9} \\ \text { New England (NE) } & 13.1 & 3.9 \\ \text { Middle Atlantic (MA) } & 19.5 & 13.5 \\ \text { East North Central (ENC) } & 6.6 & 19.7 \\ \text { West North Central (WNC) } & 16.6 & 6.4 \\ \text { Sourth Atlantic (SA) } & 9.6 & 16.5 \\ \text { Fast Solth Central (ESC) } & 12.2 & 9.6 \\ \text { West South Central (WSC) } & 4.9 & 12.0 \\ \text { Mountain (MT) } & 13.5 & 4.9 \\ \text { Pacific (PA) } & & 13.4\end{array}$

Table D.15

Projected Coal Costs and Heat Content by FPC Consuming Region

Region

New England (NE)

Middle Atlantic (MA)

East North Central (ENC)

West North Central (WNC)

South Atlantic (SA)

East South Central (ESC)

West South Central (WSC)

Mountain $(M T)$

Pacific :(PA)
Coal Pricen

(1977\$/ton)

33.89

24.11

19.37

12.76

24.36

19.83

6.04

7.15

12.35

*Assumed to be constant as a function of time.

\section{Heat Cointent}

(BTU/1D)

13,500

11,783

10,711

9,408

11,855

11,006

6,583

9,637

8,101 
Table D.16

CAPITAL COST CASH FLONS

(Fraction of Capital Spent per Ycar)

\begin{tabular}{|c|c|c|c|c|c|}
\hline \multirow[b]{2}{*}{$\begin{array}{l}\text { Years Before } \\
\text { Start-up Date } \\
\end{array}$} & \multicolumn{5}{|c|}{ Industry } \\
\hline & $\begin{array}{l}\text { Fossis } \\
\text { Plants }\end{array}$ & $\begin{array}{l}\text { Muclear } \\
\text { Plants } \\
\end{array}$ & $\begin{array}{c}\text { Fabrication \& } \\
\text { Reprocessing } \\
\text { Plants } \\
\end{array}$ & $\begin{array}{l}\text { Enrichment } \\
\text { Plants } \\
\end{array}$ & $\begin{array}{c}\mathrm{ThO}_{2} \& \\
\mathrm{U}_{3} \mathrm{O}_{8} \text { Mines }\end{array}$ \\
\hline 1 & .080 & .050 & .10 & .10 & .10 \\
\hline 2 & .230 & .090 & .45 & .20 & .20 \\
\hline 3 & .372 & .200 . & .30 & .25 & .25 \\
\hline 4 & .208 & .240 & .10 & .20 & .20 \\
\hline 5 & .085 & .187 & .05 & .15 & .15 \\
\hline 6 & .025 & .128 & .0 & .10 & .10 \\
\hline 7 & .0 & .062 & .0 & .0 & .0 \\
\hline 8 & .0 & .029 & .0 & 0 & $.0 \cdots$ \\
\hline 9 & .0 & .014 & .0 & .0 & .0 \\
\hline 10 & .0 & .0 & .0 & .0 & .0 \\
\hline
\end{tabular}

Table D.17

CAPITAL COSTS FOR FARRICATION AND REPROCESSING FACILITIES

$(\$ / \mathrm{Kg} / \mathrm{Yr} \text { throughput })^{*}$

Reactor

I,WR-US(LLE)/U

US (LF) /U-T

$\mathrm{u}$ (In:) $/ \mathrm{W} / \mathrm{Th}$

U3(IH:) $/ \mathrm{U} / \mathrm{Th}$

$\mathrm{P}_{1} / \mathrm{U}$

$\mathrm{Pu} / \mathrm{Th}$.

SSC.R-US (LF:)/U

$\mathrm{U3}(\mathrm{DBl}) / \mathrm{U} / \mathrm{Th}$ $\mathrm{Pu} / \mathrm{Th}$

INWR-US (NAT)/U

U5 (SEU) $/ U$

US (DF: $/ U / \mathrm{Th}$

U3(DE) $/ U /$ Th

$\mathrm{Pu} / \mathrm{U}$

- $\mathrm{Pu} / \mathrm{71}$

ITTGR-IIS (LI:)/UI-T

U5 (I.I.) $/ U$

$\mathrm{US}(\mathrm{DT}:) / \mathrm{d} / \mathrm{Th}$

U5(III) $/ 77_{1}$

133(118:) $/ 11 / \mathrm{TT}$

U3/Th

$\mathrm{Pu} / \mathrm{Th}$

FBR-PU-U/IJ

Pu-IJ/FI

\begin{tabular}{|c|c|c|}
\hline $\begin{array}{l}\text { Fabrication Plants } \\
\text { (511 M/Year) }\end{array}$ & & $\begin{array}{l}\text { Reprocessing Plants } \\
\text { (1500 MT/Year) }\end{array}$ \\
\hline $\begin{array}{l}127 \\
127 \\
157 \\
886 \\
507 \\
507\end{array}$ & & $\begin{array}{l}533 \\
533 \\
537 \\
537 \\
533 \\
563\end{array}$ \\
\hline $\begin{array}{l}127 \\
886 \\
507\end{array}$ & . & $\begin{array}{l}533 \\
537 \\
563\end{array}$ \\
\hline $\begin{array}{l}127 \\
127 \\
157 \\
886 \\
507 \\
507\end{array}$ & ، & $\begin{array}{l}508 \\
508 \\
512 \\
512 \\
508 \\
538\end{array}$ \\
\hline $\begin{array}{r}235 \\
235 \\
264 \\
264 \\
1237 \\
1237 \\
1237\end{array}$ & . & $\begin{array}{r}1053 \\
1053 \\
1053 \\
1053 \\
1053 \\
1053 \\
1053\end{array}$ \\
\hline $\begin{array}{l}1047 / 262 \\
1047 / 262\end{array}$ & & $\begin{array}{r}733 \\
\cdot \quad 733\end{array}$ \\
\hline
\end{tabular}

*Data provided by ORNI,; costs do not include interest during construction." 
Table D.18

Capital Costs for Enrichment Facilities,

Uranium and Thorium Mines*

Facility Type

Enrichment

Uranium Mines

Thorium Mines

\section{Capital Cost}

$300 \$ / \mathrm{Kg}$ separative work unit per year

$25 \$ / 1 b \mathrm{U}_{3} \mathrm{O}_{8}$ per year

$25 \$ / 1 \mathrm{~b} \mathrm{ThO}$, per year

$(62.6 \$ / \mathrm{Kg}$ th per year)

*Data provided by Resource Planning Associates; costs do not include interest during construction.

\section{E. DESCRIPTION OF CASES}

Four sets of scenarios, each corresponding to a different fuel cycle option, were investigated in this study. The fuel cycle options are summarized below.

\section{Once-Through (Throwoway) Cycle}

The once-through cycle stores the spent fuel without reprocessing. Reactors optimized for a throwaway cycle include an extended exposure PWR and a low-enriched HTGR design.

\section{$U-233$ and $U-235$ Recycle}

The uranium isotopes are assumed to be recycled in this option and the plutonium is assimed to be discarded. "All reactors are either natural uranium, low-enriched, or denatured designs. 
Pu-U Recycle Restricted to Secure Centers (S) and Dispersed (D) Reactors Restricted to "Denatured" Reactors

Nuclear non-proliferation considerations were addressed in this option. The plutonium fueled reactors were restricted to secure energy centers. All other reactors were either low-enriched or denatured, and therefore available to be built in dispersed areas.

\section{Eull Recycle}

The full recycle option allows both fissile plutonium and U-233 recycle, with fissile plutonium being the dominant recycle material. All reactor types included in this scenario, with the exception of the HTGR's, are operating on the plutonium/uranium cycle. Nuclear non-proliferation is not an issue in this scenario. That is, denatured reactors are not available.

For each of the four fuel cycle options, five ALPS calculations, representing five different reactor strategies, were performed. Table E.1 presents the reactors available in each of these twenty cases. The first set of cases, denoted with an $L$, assumed that the only reactor type available, in addition to the FBR's mentioned above, are PWR's. The set of cases denoted with an $S$ assumed that the throwaway PWR, the denatured U-233 fueled PWR, and the Pu/Th fueled PWR in set $\mathrm{L}$ are replaced with SSCR's on the same fuel cycle. The third set of cases, denoted with an $H$, assumed the availability of the CANDU's in addition to the standard LWR and the FBR's. The fourth set of cases, denoted: with a $G$, assumed the availability of HTGR's, in addition to the standard LWR and the FBR's. Finally, the set of cases denoted with an $A$ had all of the reactors that were included in sets $\mathrm{L}, \mathrm{S}, \mathrm{H}$, and $\mathrm{G}$ available. Thus, 
Table E.1

THORIUM ASSI:SSMENT PROGRAM REACTOR CASES

$S$ = Secure Energy Center, $D=$ Dispersed Area

\begin{tabular}{|c|c|c|c|c|c|c|c|c|c|c|c|c|c|c|c|c|c|c|c|c|}
\hline $\begin{array}{lll}\cdot & \ddots & \ddots\end{array}$ & & & yc & & & & & & & & & & & & & & Ful & & $y c 1$ & \\
\hline Reactor & $\underline{\mathbf{L}}$ & $\underline{\mathbf{S}}$ & T & $\underline{G}$ & $\underline{A}$ & $\underline{\mathrm{L}}$ & $\underline{s}$ & f & $\underline{\mathbf{G}}$ & $\underline{\mathbf{A}}$ & $\underline{L}$ & $\underline{S}$ & T & $\underline{G}$ & $\mathrm{~A}$ & $\underline{D}$ & $\underline{s}$ & 1 & $\underline{\mathbf{B}}$ & $\underline{A}$ \\
\hline I.WR - U 5 (I.F) /U & D & D & D & D & D & D & D & I & D & n & D & D & D & D & D & D & D & 1 & D & D \\
\hline I.WR-US (I.F) /U-T & D & - & - & - & D & - & - & .1 & - & - & - & - & - & - & - & - & - & . & - & - \\
\hline L.WR - US (DF: ]/U/Th & - & - & - & - & - & D & D & - & - & D & $\mathbf{D}$ & D & - & - & $\mathbf{D}$ & - & $\therefore$ & . & - & - \\
\hline L.WR-U3 (DE] /U/'Th & - & - & - & - & - & D & - & - & - & D & D & $\because$ & - & - & D & - & - & . & - & - \\
\hline 1.WR $-\mathrm{Pu} / \mathrm{U}$ & - & - & - & - & - & - & - & - & - & - & - & - & - & - & $\therefore-$ & $\mathbf{S}$ & $\mathbf{S}$ & . & - & $\mathbf{S}$ \\
\hline LWR-Pu/Th & - & - & - & - & - & - & - & - & - & - & $\mathbf{S}$ & $=$ & - & - & $\mathbf{S}$ & - & - & . & - & - \\
\hline SSCR-US (LE) /U & - & D & - & - & D & - & D & - & - & D & - & $\mathrm{D}$ & - & - & D & - & D & . & & D \\
\hline SSCR-U3(DE)/U/Th & - & - & - & - & & - & D & - & - & D & - & D & - & - & D & - & - & 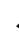 & - & - \\
\hline SSCR-Pu/Th & - & - & - & - & - & - & - & - & - & - & $\because$ & $\mathbf{s}$ & - & - & $\mathbf{S}$ & - & - & . & - & - \\
\hline IIWR - U 5 (NAT)/U & - & - - & D & - & D & - & - & D & - & D & - & - & D & - & D & - & - & 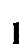 & - & D \\
\hline IIWR - U5 (SEU) /U & - & - & $\bar{D}$ & - & D & 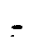 & $=$ & I & - & D & - & - & D & - & D & - & - & 1 & - & D \\
\hline IIWR - US (DE)/U/Th & - & - & - & - & - & - & - & D & - & D & -. & - & D & - & D & - & - & 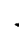 & - & - \\
\hline IIIVR- 113 (DI: ) $/ U / \mathrm{Th}$ & . & - & - & - & - & - & - & I & - & D & - & - & D & - & D & - & - & 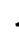 & - & - \\
\hline HWR - Pú/U & - & - & - & - & - & - & - & . & - & - & $=$ & - & - & - & - & - & - & $s$ & - & $\mathbf{S}$ \\
\hline lillR - Pu/Th & - & - & - & - & - & - & - & . & - & - & - & - & S & - & $s$ & - & - & 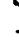 & - & - \\
\hline IITGR-U.5 (I.E) /U-T & - & .- & - & D & D & - & - & . & - & - & - & :- & - & - & - & - & - & . & - & - \\
\hline ITGR-US (J.E)/U & - & - & - & $=$ & - & 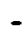 & - & & D & D & - & - & - & D & D & - & - & 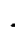 & D & D \\
\hline IITGR-US (DE)/U/Th & - & - & - & - & - & - & $\cdot$ & . & D & D & - & - & $=$ & D & D & - & - & . & - & - \\
\hline IITGR-US(HI) /Th & - & - & - & - & - & - & - & & - & - & 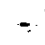 & - & $\therefore$ & - & - & - & - & 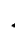 & $\mathbf{s}$ & $\mathbf{S}$ \\
\hline HTGR-H3 (J) $) / U / T h$ & - & - & - & & - & - & - & . & D & D & - & - & - & D & D & - & - & . & - & - \\
\hline IITGR-U3/Th & - & - & - & - & & & & & & 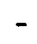 & - & - & - & - & - & $=$ & - & . & $\mathbf{S}$ & $\mathbf{S}$ \\
\hline IITGR-Pu/Th & - & - & - & & & $\because$ & - & 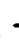 & - & - & $=$ & - & - & $\mathbf{S}$ & $\mathbf{S}$ & - & - & r & $\mathbf{S}$ & $\mathbf{S}$ \\
\hline$P B R-P u-U / U$ & - & - & - & & - & & 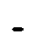 & & - & & - & - & - & - & - & $\mathbf{s}$ & $\mathbf{S}$ & 8 & $\mathbf{S}$ & $\mathbf{S}$ \\
\hline FBR-PU-U/Th & - & - & - & - & - & - & - & & - & - & $\mathbf{s}$ & $\mathrm{s}$ & s & $\mathbf{S}$ & $s$ & - & & & - & - \\
\hline
\end{tabular}


in this case, LWR's, SSCR's, CANDU's, HTGR's, and FBR's competed directly against each other (and against fossil plants) for a share of the market.

F. RESULTS

The results of this study are shown for the following key variables-total system costs, levelized system power costs, total capacity built, coal consumption, uranium ore $\left(\mathrm{U}_{3} \mathrm{O}_{8}\right)$ consumption, maximm enrichnent requirements and $\mathrm{U}_{3} \mathrm{O}_{8}$ and enrichment utilization. Also reported are the installed nuclear capacity in year 2026 and levelized power costs in year 2025 for each reactor type available. Capital requirements for the various segments of the nuclear industry and the fossil generation plants are shown in the form of cash flow tables. Reactor and system material requirements are presented graphically. in Appendix A to show the uranium ore, enrichment, fabrication, reprocessing, and fissile material requirements for the year 2035 per gigawatt of installed nuclear capacity. Because uranium ore availability and cost projections may vary, the above-mentioned results are presented for both small and large $\mathrm{U}_{3} \mathrm{O}_{8}$ supply assumptions.

Total Systems Costs

The first key variable shown in Tables F.1 through F.5 is the presentworthed total system costs of electrical generation from 1977 through 2050. This variable which the ALPS model minimizes is reported for three discount rates-- $-41 / 2,71 / 2$ and $10 \%$ per year. Comparison of all four fuel cycle options for the five different reactor strategies shows the same general trends. Note the slight decrease in systems cost at $41 / 2 \%$ discount rate (0-17 billion dollars) in choosing the U-233 and U-235 recycle option over the once-through (throwaway) option. Also, one can see that choosing either the restricted or the full recycle option over the throwaway option results 
Table F.1

SUMMARY OF THORILM ASSESSMENT PROGRAM STUDY RESULTS

SMALL $\mathrm{U}_{3} \mathrm{O}_{8}$ SUPPLY

Fuel Cycle Options

\begin{tabular}{|c|c|c|c|}
\hline $\begin{array}{l}\text { Once-Through } \\
\text { (Throwaway) } \\
\text { Cycle } \\
\text { (l) }\end{array}$ & $\begin{array}{c}\text { U-233 \& } \\
\text { U-235 } \\
\text { Recycle } \\
\end{array}$ & $\begin{array}{c}\text { Restricted } \\
\text { Recycle }\end{array}$ & $\begin{array}{c}\text { Full } \\
\text { Recycle }\end{array}$ \\
\hline & & & \\
\hline $\begin{array}{r}1804 \\
787 \\
479\end{array}$ & $\begin{array}{r}1806 \\
791 \\
483\end{array}$ & $\begin{array}{r}1703 \\
760 \\
469\end{array}$ & $\begin{array}{r}1701 \\
758 \\
468\end{array}$ \\
\hline
\end{tabular}

System Costs ( $\$ B$ )

1977 through 2050

Discounted at

$4 \frac{1}{7} 1 / 2 \%$
$10 \%$

479

18.8

18.9

18.2

18.3

Levelized System

2025 (mills/kwhr)

Cumulative Capacity

Built (GWe) through

2050

Nuclear.

Fossil

$\begin{array}{rrrr}705(13 \%) & 933(18 \%) & 2597(49 \%) & .2663(50 \%) \\ 4611(87 \%) & 4382(82 \%) & 2719(51 \%) & 2653(50 \%)\end{array}$

Annual Coal Consunmp-

tion in 2025 ( $10^{6}$ tons)

5220

4790

2910

.3150

Cumulative $\mathrm{U}_{3} \mathrm{O}_{8} \mathrm{Con}$ -

sumption (105 tons)

through

2026

.2050

2.92

3.42

2.88

3.13

3.68

4.33

3.56

4.60

Maximum Annual Enrich-

ment Requirement

through 2050 (106 Siv/

yr)

54

72

80

73

$\mathrm{U}_{3} \mathrm{O}_{8}$ Utilization $(1)$

(ton $\mathrm{U}_{3} \mathrm{O}_{8} / \mathrm{GWe}$ )

5033

3403

1684

1899

Enrichment Utilization ( 2 )

(10 SWU/GWe)

3.00

3.13

1.20

1.08

(1) Cumlative $\mathrm{U}_{3} \mathrm{O}_{8}$ consumed through 2050 (including forward cumittment) per cumulative nuclear capacity built through 2050

(2) Cimulative enrichment requirements through 2050 per cumulative nuclear capacity built through 2050 
Table F.2

SUMMARY OF THORILM ASSESSIENT PROGRAM STUDY RESULTS SMALL $\mathrm{U}_{3} \mathrm{O}_{8}$ SUPPLY

System Costs $(\$ B)$

\begin{tabular}{cccc}
\multicolumn{4}{c}{ Fuel Cycle Options } \\
$\begin{array}{c}\text { Once-Through } \\
\text { (Throwaway) } \\
\text { Cycle }\end{array}$ & $\begin{array}{c}\text { U-235 } \\
\text { Recycle }\end{array}$ & $\begin{array}{c}\text { Restricted } \\
\text { Recycle }\end{array}$ & $\begin{array}{c}\text { Ful1 } \\
\text { Recycle }\end{array}$ \\
\cline { 1 - 1 } & $\frac{(S)}{(S)}$ & $\frac{(S)}{(S)}$ & \\
1807 & 1797 & 1681 & 1688 \\
789 & 790 & 754 & 755 \\
480 & 483 & 467 & 468
\end{tabular}

Levelized System

Power costs in

2025 (mills/kwhr)

18.9

18.9

17.8

18.0

Cumulative Capacity

Built (Giv) through

2050

Nuclear

Fossil

$745(14 \%)$

$1143(22 \%)$

$4173(78 \%) \quad 2148(408)$

$3037(57 \%)$

$4571(86 \%)$

5170

4330

2340

2680

Annual Coal Consynty-
tion in 2025 (10 tons)

Cumulative $\mathrm{U}_{3} \mathrm{O}_{8}$ Con-

sumption (106 tons)

through

2026

2.81

3.40

3.01

3.27

3.52

4.34

3.50

2050

Maximm Annual Enrich-

ment Requirement

through 2050 ( $10^{6}$ ST:U/

yr)

47

78

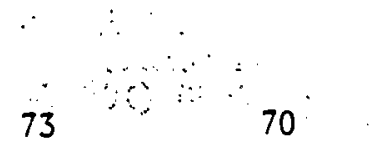

$\mathrm{U}_{3} \mathrm{O}_{8}$ Utilization $(1)$

(ton $\mathrm{U}_{3} \mathrm{O}_{8} / \mathrm{G}$ e)

4892

2896

1392

1744

Enrichment Utilization (2)

(106 SWU/GWe)

2.65

2.39

0.96

0.94

(1) Cumulative $\mathrm{U}_{3} \mathrm{O}_{8}$ consumed through 2050 (including forward committment) per cimulative nuclear eapacity built through 2050

(2) Cumulative enrichment requirements through 2050 per cumlative nuclear. capacity built through 2050 
Table F.3

SUMMARY OF THORILM ASSESSUENT PROGRAM STUDY RESULTS SMALL $\mathrm{U}_{3} \mathrm{O}_{8}$ SUPPLY

System Costs ( $\$ B$ )

\begin{tabular}{|c|c|c|c|}
\hline \multicolumn{4}{|c|}{ Fuel Cycle Options } \\
\hline $\begin{array}{l}\text { Once-Through } \\
\text { (Throwaway) } \\
\text { Cycle }\end{array}$ & $\begin{array}{c}\text { U-233 \& } \\
\text { U-235 } \\
\text { Recycle }\end{array}$ & $\begin{array}{l}\text { Restricted } \\
\text { Recycle }\end{array}$ & $\begin{array}{l}\text { Full } \\
\text { Recycle }\end{array}$ \\
\hline
\end{tabular}

1977 through 2050

Discounted at

$41 / 2 \%$

$71 / 29$

1811

790

$10 \%$

480

1810

789

481

1732
769

1716

766

473

Levelized System

Power Costs in

2025 (mills/kwhr)

18.8

18.9

18.2

18.0

Cumulative Capacity

Built (GWe) through

2050

Nuclear

Fossil

$\begin{array}{rrrr}699(13 \%) & 830(16 \%) & 3108(58 \%) & 3598(68 \%) \\ 4617(87 \%) & 4486(84 \%) & 2208(42 \%) & 1718(32 \%)\end{array}$

Annual Coal Consunm-

tion in 2025 ( $10^{6}$ tons)

5350

5030

3020

3090

Cumulative $\mathrm{U}_{3} \mathrm{O}_{8}$ Con-

surmtion (106 tons)

through

2026

2050

2.79

2.76

3.23

3.24

3.25

3.25

3.60

3.62

Maximm Annual Enrich-

ment Requirement

through 2050 ( $10^{6} \mathrm{sWU} /$

yis)

49

58

70

70

$\mathrm{U}_{3} \mathrm{O}_{8}$ Utilization $(1)$

(ton $\mathrm{U}_{3} \mathrm{O}_{8} /$ Give)

4942

4078

1164

1013

Enrichment Utilization (2)

(10 $\mathrm{F}$ SiU/Give)

2.81

0.83

0.72

(1) Cumslative $\mathrm{U}_{3} \mathrm{O}_{8}$ consumed through 2050 (including forvard committment) per cumulative nuclear capacity built through 2050

(2) Cumulative enrichment requirements through 2050 per cumulative nuclear capacity built through 2050 
Table F.4

SUMMARY OF THORIUM ASSESSTENT PROGRAM STUDY RESULTS

SMALL U $\mathrm{U}_{3} \mathrm{O}_{8}$ SIIPPLY

System Costs ( $\$ B$ )

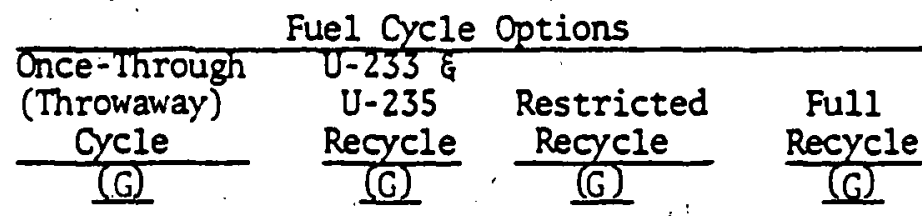

1977 through 2050

Discounted at

$41 / 2 \%$

$71 / 2 \%$

$10 \%$

$\begin{array}{rrrr}1810 & 1793 & 1697 & 1685 \\ 790 & 787 & 760 & 757 \\ 481 & 481 & 470 & .769\end{array}$

Levelized System

Power Costs in

2025 (mills/kwhr)

18.9

18.7

18.0

17.7

Cumulative Capacity

Built (GVe) through

2050

Nuclear

Fossil

$\begin{array}{rlll}701(13 \%) & 1301(24 \%) & 2791(53 \%) & 3212(60 \%) \\ 4615(87 \%) & 4015(76 \%) & 2525(47 \%) & 2104(40 \%)\end{array}$

Annual Coal Consunty-

tion in 2025 (106 tons)

5330

4090

2560

2330

Cumulative $\mathrm{U}_{3} \mathrm{O}_{8}$ Con-

sumption (106 tons)

through

2026

2050

2.74

3.01

3.63

4.22

3.41

3.25

3.93

4.75

Maximm Annual Enrich-

ment Requirement

through 2050 (106 siv/

yr)

49

76

80

79

$\mathrm{U}_{3} \mathrm{O}_{8}$ Utilization $(1)$

(ton $\mathrm{U}_{3} \mathrm{O}_{8} / \mathrm{GWe}$ )

5005

3076

1524

1550

Enrichment Utilization (2)

(10 5 SW/GWe)

2.89

2.50

1.09

1.24

(1) Cumulative $U_{3} O_{8}$ consumed through 2050 (including forvard committinent) per cumulative nưclear capacity built through 2050

(2) Cumulative enrichment requirements through 2050 per cumulative nuclear capacity built through 2050 
Table F.5

SUAMARY OF THORILM ASSESSIENT PROGRAM STUDY RESULTS SMALL $U_{3} 0_{8}$ SUPPLY

System Costs ( $\$ B)$

1977 through 2050

Discounted at

$41 / 2 \%$

$71 / 2 \%$

108

\begin{tabular}{|c|c|c|c|}
\hline \multicolumn{4}{|c|}{ Fuel Cycle Options } \\
\hline $\begin{array}{l}\text { Once-Through } \\
\text { (Throwaway) } \\
\text { Cycle } \\
\text { (A) }\end{array}$ & $\begin{array}{l}\text { U-233 } \\
\text { U-235 } \\
\text { Recycle } \\
\text { (A) }\end{array}$ & $\begin{array}{l}\text { Restricted } \\
\text { Recycle }\end{array}$ & $\begin{array}{l}\text { Full } \\
\text { Recycle } \\
\text { (A) }\end{array}$ \\
\hline $\begin{array}{r}1802 \\
786 \\
479\end{array}$ & $\begin{array}{r}1789 \\
788 \\
482\end{array}$ & $\begin{array}{r}1676 \\
-752 \\
466\end{array}$ & $\begin{array}{r}1676 \\
752 \\
466\end{array}$ \\
\hline
\end{tabular}

Levelized System

Power Costs in

2025 (mills/kwhr)

18.9

18.8

17.8

17.8

Cumulative Capacity Built (GVe) through 2050

\section{Nuclear \\ Fossil}

Annual Coal Consump-

tion in 2025 ( $10^{6}$ tons)

Cumulative $\mathrm{U}_{3} 0_{8}$ Con-

surmption (106 tons)

through

$$
2026
$$$$
2050
$$

Maximum Annual Enrichment Requirement through 2050 ( $10^{6}$ SWU/ yr)

$\mathrm{U}_{3} \mathrm{O}_{8}$ Utilization $(1)$

(ton $\mathrm{U}_{3} \mathrm{O}_{8} /$ GWe)

Enrichment Utilization (2)

(10 $\left.10^{6} \mathrm{SWU} / \mathrm{GWe}\right)$
2.94

3.52

3.05

3.83

3.58

4.71

3.49

4.79

$\begin{array}{rll}773(15 \%) & 1342(25 \%) & 3181(60 \%) \\ 4543(85 \%) & 3974(75 \%) & 2135(40 \%)\end{array}: \begin{aligned} & 3159(59 \%) \\ & 2157(41 \%)\end{aligned}$

$5160 \quad 3980 \quad 2250 \quad 2230$

52

1863

2902

1524

1571

2.71

2.44

1.03

1.21

(1) Cumlative $\mathrm{U}_{3} \mathrm{O}_{8}$ consumed through 2050 (including forward committment) per cumlative nuclear capacity built through 2050

(2) Cumulative enrichment requirements through 2050 per cumulative nuclear capacity built through 2050 
in a much larger decrease in system costs of 100-125 billion dollars. Total system costs assuming a large $\mathrm{U}_{3} \mathrm{O}_{8}$ supply (Tables F.15 through F.19) exhibit the same relative differences, however, the less expensive uranium ore reduces all total systems costs by 60-70 billion dollars. In comparing five different reactor strategies-L(LWR's only), S(LWR's and SSCR's), H(LWR's and CANDU's), G(LWR's and HTGR's) and A(all reactors), in addition to FBR's in the appropriate fuel cycle options, the economic benefits of deploying specific reactor types under the four different fuel cycle options become apparent. For the throwaway fuel cycle option, the LWR optimized for a throwaway cycle with extended exposure has a slight economic edge ( $\leq 7$ billion dollars) over all other reactor strategies combined with the standard low-enriched LWR. For both the U-233 and U-235 recycle option and the full recycle option (includes FBR's), the HTGR reactor strategy provides slightly larger benefits ( 15 billion dollars) than the LWR reactor strategy. The SSCR reactor strategy does, however, result in nearly the same benefits in the full recycle fuel option. For the restricted recycle option (includes FBR's), the SSCR reactor strategy provides the largest benefit of 22 billion dollars relative to the LWR reactor strategy. The CANDU reactors (reactor strategy $H$ ), although their. fuel cycle costs are lower, are penalized by the higher capital costs associated with a heavy water inventory--resulting in higher total system. costs than the LWR reactor strategy for all four fuel cycle options. As one might expect, the reactor strategy A employing all four reactor types (LWR, SSCR, CANDU, and HTGR), the total system costs are slightly less than any strategy employing only one reactor type. 


\section{Levelized System Power Costs}

The levelized system power costs shown in the tables represent the average power costs in mills/kwhr for all nuclear and fossil power plants operating in the year 2025. Relative to the throwaway fuel cycle option, recycle of $U-23 j$ and $U-235$ without FBR's reduces power costs very slightly (0.1 mills/kwhr) if any at al1. Results shown indicate that only through utilization of either restricted or full recycle with FBR's will the average power cost be reduced appreciably (1-2 mills/kwhr).

\section{Cumulative Capacity Built}

The split between nuclear and fossil cumulative capacity built through 2050. is also reported both in gigawatt of electrical capacity (GWe) and the percentage of the total generation market. The reduction in total system costs and average system power costs correlates very well with the changes in market penetration attained by nuclear power plants. Nuclear market penetration for small $\mathrm{U}_{3} \mathrm{O}_{8}$ supply increases from $15 \%$ to $25 \%$ if U-233 and U-235 recycle is employed instead of the throwaway cycle, and likewise from $20 \%$ to $40 \%$ for large $\mathrm{U}_{3} \mathrm{O}_{8}$ supply. If restricted or full recycle is employed nuclear market penetration increases for small $U_{3} O_{8}$ supply to $50 \%$ - $60 \%$ and for large $U_{3} \mathrm{O}_{8}$ supply, as large as $60 \%$ - $70 \%$ nuclear market penetration results.

\section{$U_{3} \underline{O}_{8}$ Consumption and Utilization-Enmichment Utilization}

Cumulative $\mathrm{U}_{3} \mathrm{O}_{8}$ consumption results reported in the tables show that for the throwaway and $U-233$ and $U-235$ recycle options $3-4$ million tons of $\mathrm{U}_{3} \mathrm{O}_{8}$ are consumed. For restricted and full recycle options slightly more (4-5 million tons) $U_{5} 0_{8}$ is consumed for production of fissile material to be used in FBR's. An important point to note is the behavior of $\mathrm{U}_{3} \mathrm{O}_{8}$ 
utilization which is a measure of the quantity of $\mathrm{U}_{3} \mathrm{O}_{8}$ required to support one gigawatt of electrical generating capacity. Although more $\mathrm{U}_{3} \mathrm{O}_{8}$ is consumed for both recycle options with breeders, the $\mathrm{U}_{3} \mathrm{O}_{8}$ utilization is reduced from $3000-5000$ tons $U_{j} 0_{8}$ per Give to $1400-1900$ tons $U_{j} 0_{8}$ per GWe.

Enfichment utilization--the quantity of enrichment required per gigawatt--behaves similarly to $\mathrm{U}_{5} \mathrm{O}_{8}$ utilization. Enrichment requirements per gigawatt increases by a factor of 3-4 for fuel cycle options without FBR's.

Installed Nuclear Caracity - Levelized Power Costs i::

A "srapshot in time" of instalied capacity (GWe) of each reactor in the year 2026 and the levelized power costs (milis/kwhr) for each reactor if startupi occurs in year 2025 are presented in Tables F.6 through F.10. for small $U_{3} O_{8}$ supply and Tables F.20 through $F .24$ for large $U_{3} O_{8}$ supply. Looking at the. four fuel cycle options with the LWR reactor strategy (Table F.6), note that the LWR optimized for throwaway does take over the nuclear market but is still limited by high cost $\mathrm{U}_{3}{ }^{0} 8$ in the later years. Allowing introduction of U-233 and U-235 recycle in LWR's nearly doubles nuclear capacity. Much larger changes (4-5 times as much nuclear capacity) result when either restricted or full recycle of Pu is allowed. The large increase in installed capacity of the standard low-enriched LiWR provides adequate fissile Pu to be recycled in FBR's. About '15\% of the generating capacity is also provided by LWR's recycling U-2jJ in the restricted recyele option. In the full recycle option about $17 \%$ of the muclear capacity is provided by LWR's burning fissile Pu not required by FBR's.

If the SSCR reactor strategy is pursued, results shown in Table F.7 are obtained. The SSCR's because of their better fuel utilization increase 
Table F.6

THORILIN ASSESSMIENT PROGRAM RESULTS

Small $\mathrm{U}_{3} \mathrm{O}_{8}$ Supply

INSTALLLD CAPACITY (GWe) in YEAR 2026/LEVELIZED POWER COSTS (Mills/Kwhr) in YEAR 2025

Once-Through

(Throwaway)

Cycle

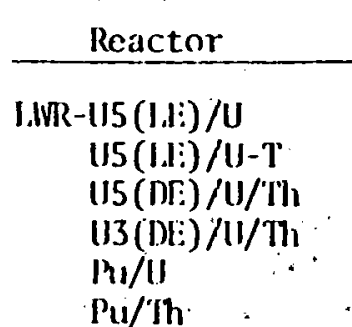

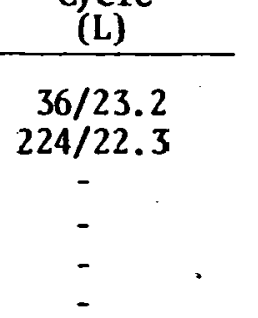

SSCR-U5(LI:)/II

(13(1)I:)/U/'Ih

$\mathrm{m} / \mathrm{I} / \mathrm{h}$

I IIVR-UL(NAT)/II

U5 (Sl:U) $/ U$

IIS(I) $\mathrm{T}) / \mathrm{U} / \mathrm{Ih}$

U3(II) $/ \mathrm{H} / \mathrm{Ih}$

$\mathrm{Mu} / \mathrm{U}$

$\mathrm{Iu} / \mathrm{Th}$

IITCR-UL(LE)/U-T

IIS(LI:) $/ U$

U5(D): $/ \mathrm{H} / \mathrm{Th}$

US(IIE)/:Ih

II3(I) $) / U / T h$

. U3/Th

$\mathrm{Pu} / \mathrm{Ih}$

IBBR-PUI-II/II

In- 1 /'Ih

lossil

1934

U-233 \& U-235

Recycle

(L)

$113 / 21.6$

$189 / 22.5$

$157 / 20.0$

$-$

$-$

$-$

Nuclcar
261
Restricted :

Recycle

(L)

$594 / 20.7$

$0 / 23.2$

$190 / 19.6$

$52 / 22.1$

Full Recycle

(L)

$513 / 20.8$

$-$

$196 / 19.5$

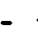

$-$

-

$-$

-
$444 / 18.4$

$408 / 19.4^{5}$

951

1244
1042

1153 
Table F.7

THORIUM ASSESSMENT PROGRAM RESULTS

Small $\mathrm{U}_{3} 0_{8}$ Supply

INSTALITD CAPACITY (GWe) in YEAR 2026/IEVELIZED POIVER COSTS (Mills/Kwhr) in YFAR 2025

\begin{tabular}{|c|c|c|c|c|}
\hline Reactor & $\begin{array}{c}\text { Once-Through } \\
\text { (Throwaway) } \\
\text { Cycle } \\
\text { (S) }\end{array}$ & $\begin{array}{c}\text { U-233 \& U-235 } \\
\text { Recycle } \\
\text { (S) } \\
\end{array}$ & $\begin{array}{c}\text { Restricted } \\
\text { Recycle } \\
\text { (S) } \\
\end{array}$ & $\begin{array}{l}\text { Full Recycle } \\
\text { (S) }\end{array}$ \\
\hline LIVR-US (LE)/U & $95 / 23.1$ & $82 / 22.1$ & $170 / 20.9$ & $161 / 21.6$ \\
\hline US (ILE)/U-T & - & - & - & - \\
\hline IIS(DE)/U/Th & - & $273 / 22.7$ & $2 / 23.2$ & - \\
\hline $113(\mathrm{DE}) / \mathrm{U} / \mathrm{Th}$ & - & - & - & - \\
\hline$P_{1} / / \mathrm{J}$ & - & - & - & $207 / 19.6$ \\
\hline $\mathrm{Pu} / \mathrm{Th}$ & - & - & - & - \\
\hline SSCR-UJ (LF)/U & $197 / 21.8$ & $0 / 21.3$ & $495 / 19.5$ & $454 / 20.0$ \\
\hline II3(DE)/U/Th & - & $306 / 20.5$ & $294 / 19.7$ & - \\
\hline $\mathrm{I}$ 'u/ $\mathrm{Th}$ & - & - & $72 / 20.8$ & - \\
\hline InNR-U5(NAI)/U & - & - & - & - \\
\hline US(SIEU) $/ U$ & - & - & - & - \\
\hline $115(\mathrm{DI}) / \mathrm{U} / \mathrm{Th}$ & - & - & - & - \\
\hline U13(DI:)/U/Th & - & - & - & - \\
\hline $\mathrm{Pu} / \mathrm{U}$ & - & - & - & - \\
\hline $\mathrm{Ii} / \mathrm{Th}$ & - & - & - & - \\
\hline IfICR-U5 (LE)/U-T & - & - & $\sin$ & $\therefore-$ \\
\hline U5(LE)/U & - & - & $\because=$ & औन \\
\hline US (Dr:) $/ \mathrm{UJ} / \mathrm{Th}$ & - & - & $y^{\prime}$ & - \\
\hline U5 (In:)/Ih & - & - & $\because \quad \therefore$ & - \\
\hline U3(DF:)/U/Th & - & - & - & - \\
\hline U3/Th & - & - & - & $\because$ \\
\hline $\mathrm{ML} / \mathrm{Ih}$ & - & - & - - & $\cdot-$ \\
\hline $\mathrm{I} B \mathrm{BR}-\mathrm{Pu}-\mathrm{U} / \mathrm{U}$ & - & - & - & $511 / 18.5$ \\
\hline $121-1 I / 1 h$ & $\because "-$ & - & $420 / 19.3$ & \\
\hline llossill & 1903 & 1534 & 742 & 862 \\
\hline Nuclear & 292 & 661 & 1453 & 1333 \\
\hline
\end{tabular}


Table F.8

THORIUM ASSESSMENT PROGRAM RESULTS

Snall U $\mathrm{U}_{3}$ Supply

INSTAILLD CAPACITY (GWe) in YEAR 2026/LEVELIZED POIVR COSTS (Niils/Kwhr) in YEAR 2025

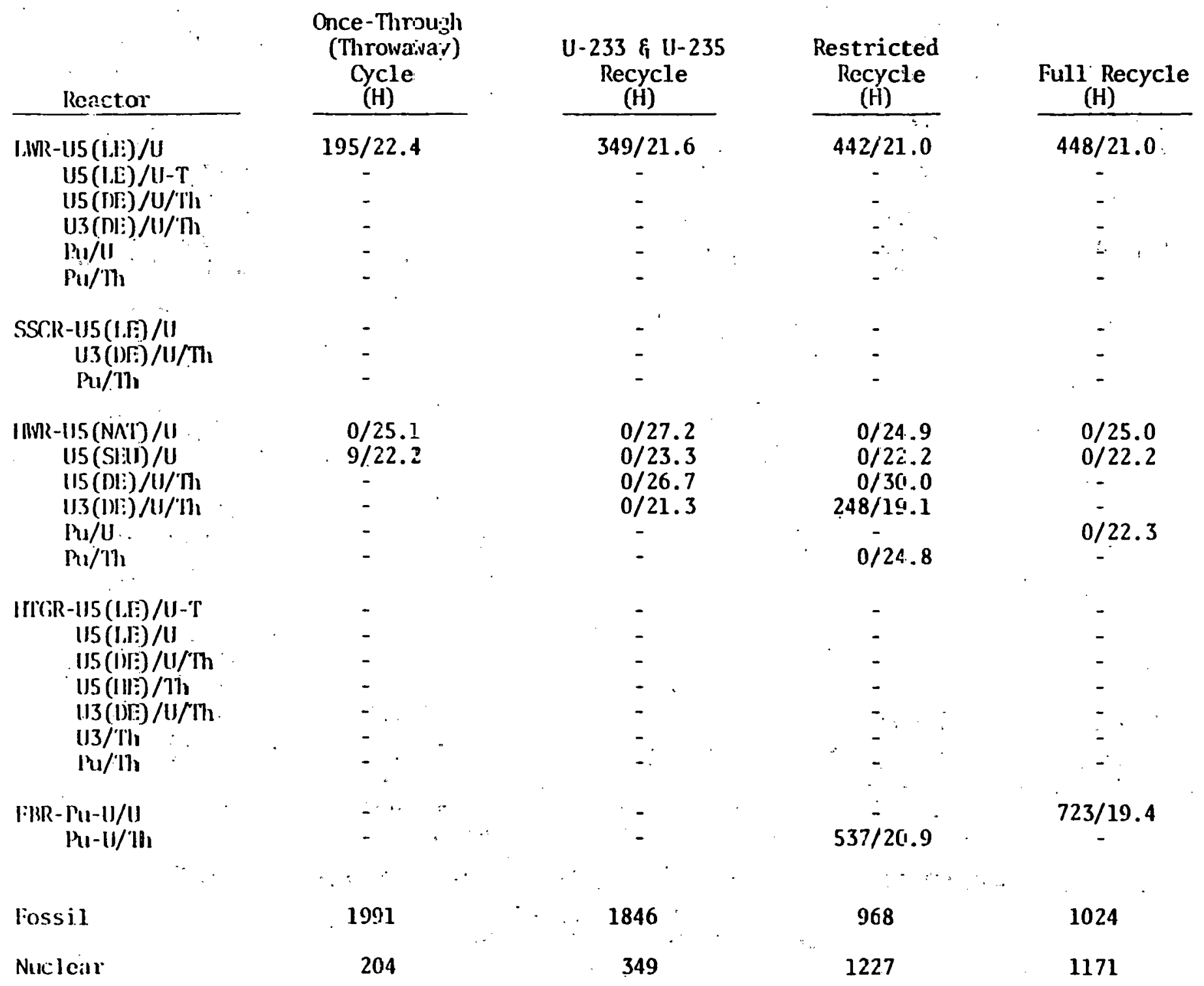


Table F.9

\section{TIORILM ASSESSAENT PROGRAM RESULTS}

$$
\text { Small } \mathrm{U}_{3} \mathrm{O}_{8} \text { Supply }
$$

INSTALLED CAPACITY (GNe) in YEAR 2026/LIVELIZIED POIVIR COSTS (Mi1ls/Kwhr) in YEAR 2025

\begin{tabular}{|c|c|c|c|c|}
\hline $\begin{array}{c}\cdots \\
\cdots \text { Reactor }\end{array}$ & $\begin{array}{c}\text { Once-Through } \\
\text { (Throwanay) } \\
\text { Cycle } \\
\text { (G) }\end{array}$ & $\begin{array}{c}\text { U-233 \& U-235 } \\
\text { Recycle } \\
\text { (G) }\end{array}$ & $\begin{array}{c}\text { Restricted } \\
\text { Recycle } \\
(G)\end{array}$ & $\begin{array}{l}\text { Full Recycle } \\
\text { (G) }\end{array}$ \\
\hline $\begin{array}{l}\text { UMR-U5(LE)/U } \\
\text { U5(LF)/U-T } \\
\text { US (D:) /U/Th } \\
\text { U3 (DE)/U/Tו } \\
\text { Pu/U } \\
\text { Pu/Th }\end{array}$ & $\begin{array}{c}141 / 22.5 \\
- \\
- \\
- \\
- \\
-\end{array}$ & $\begin{array}{c}125 / 22.7 \\
- \\
- \\
- \\
- \\
-\end{array}$ & $\begin{array}{c}566 / 20.6 \\
- \\
- \\
- \\
- \\
-\end{array}$ & $\begin{array}{c}230 / 21.2 \\
- \\
- \\
- \\
- \\
-\end{array}$ \\
\hline $\begin{array}{c}\text { SSCR-U5(LE) } / U \\
\text { U3(DE)/U/Th } \\
\mathrm{MU} / \mathrm{Th}\end{array}$ & $\begin{array}{l}- \\
-\end{array}$ & $\begin{array}{l}- \\
-\end{array}$ & - & - \\
\hline $\begin{array}{l}\text { IIIR-U5(NAT)/U } \\
\text { U5 (SLU)/U } \\
\text { II5(DE)/U/Th } \\
\text { U3 (DF)/U/Th } \\
\text { Pu/U } \\
\text { Pu/Th }\end{array}$ & $\begin{array}{l}- \\
- \\
- \\
- \\
-\end{array}$ & $\begin{array}{l}- \\
- \\
- \\
-\end{array}$ & $\begin{array}{l}- \\
- \\
- \\
-\end{array}$ & $\begin{array}{r}: \\
: \\
- \\
- \\
- \\
- \\
-\end{array}$ \\
\hline $\begin{array}{c}\text { IITGR-IJS(LI:)/U-T } \\
\text { U5(LE)/UI } \\
\text { U5(DE)/U/Th } \\
\text { US(IIE)/Th } \\
\text { U3(UE) } / U / T h \\
\text { U3/Th } \\
\text { Pu/Th }\end{array}$ & $\begin{array}{c}75 / 21.7 \\
- \\
- \\
- \\
- \\
- \\
-\end{array}$ & $\begin{array}{c}- \\
0 / 21.7 \\
487 / 20.9 \\
- \\
136 / 20.9 \\
- \\
-\end{array}$ & $\begin{array}{c}0 / 21.1 \\
0 / 20.7 \\
- \\
273 / 19.6 \\
45 / 23.4\end{array}$ & $\begin{array}{c}\overline{0} / 21.5 \\
- \\
427 / 18.8 \\
- \\
291 / 19.1 \\
100 / 19.1\end{array}$ \\
\hline $\begin{array}{r}\mathrm{FBR}-\mathrm{Pu}-\mathrm{U} / \mathrm{U} \\
\mathrm{Pu}_{\mathrm{I}}-\mathrm{U} / \mathrm{Th}_{\mathrm{L}}\end{array}$ & - & - & $493 / 21.1$ & $360 / 18.0$ \\
\hline lossil & 1979 & 1447 & 818 & 742 \\
\hline Nuclear & 216 & 748 & 1377 & 1453 \\
\hline
\end{tabular}


Table F.10

TIORILM ASSI:SSMENT PROGRAM RESULTS

Sinal1 $\mathrm{U}_{\mathbf{3}} \mathbf{0}_{8}$ Supply

INSTAI.LED CAPACITY (GIV) in YEAR 2026/LEVELIZED POIVER COSTS (Mills'/KWhr) in YEAR 2025

\begin{tabular}{|c|c|c|c|}
\hline $\begin{array}{c}\text { (Throwawa): } \\
\text { Cycle } \\
\text { (A) }\end{array}$ & $\begin{array}{c}\text { U-233\& \&-235 } \\
\text { Recycle } \\
\text { (A) } \\
\end{array}$ & $\begin{array}{c}\text { Restricted } \\
\text { Fiecycle } \\
(\mathrm{A}) \\
\end{array}$ & $\begin{array}{l}\text { Full Recycle } \\
\text { (A) }\end{array}$ \\
\hline $\begin{array}{l}36 / 23.2 \\
82 / 22.3\end{array}$ & $73 / 22.6$ & $\begin{array}{c}105 / 20.7 \\
-\end{array}$ & $\begin{array}{c}150 / 21.6 \\
-\end{array}$ \\
\hline- & $\begin{array}{r}116 / 23.0 \\
0 / 23.2\end{array}$ & $\begin{array}{l}2 / 22.8 \\
0 / 20.8\end{array}$ & - \\
\hline - & - & - & $122 / 19.9$ \\
\hline - & 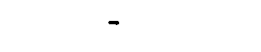 & $41 / 21.2$ & - \\
\hline $140 / 21.9$ & $0 / 21.8$ & $579 / 19: 3$ & $148 / 20.0$ \\
\hline- & 0 & $21 / 21.0$ & - \\
\hline $0 / 25.7$ & $0 / 28.1$ & $0 / 23.8$ & $0 / 24.7$ \\
\hline $0 / 22.6$ & $0 / 24.0$ & $0 / 21.7$ & $0 / 22.4$ \\
\hline - & 0 & $0 / 24.4$ & - \\
\hline - & - & & $0 / 22.8$ \\
\hline - & - & $0 / 22.8$ & - \\
\hline $0 / 22.3$ & - & - & . \\
\hline - & $0 / 21.7$ & $0 / 21.1$ & $0 / 21.8$ \\
\hline - & $372 / 20.8$ & $0 / 20.8$ & - \\
\hline - & - & - & $444 / 19.1$ \\
\hline$-\cdot$ & $244 / 20.7$ & $56 / 19.3$ & - \\
\hline- & - & - & $263 / 19.6$ \\
\hline- & - & b/20.4 & $30 / 19.8$ \\
\hline - & - & - & $353 / 18.3$ \\
\hline - & 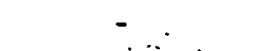 & $402 / 18.6$ & - \\
\hline 1937 & 1390 & 756 & 685 \\
\hline 258 & 805 & 1439 & 1510 \\
\hline
\end{tabular}

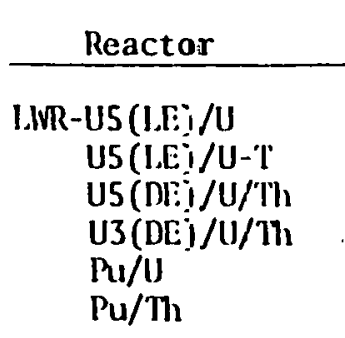

SSCR-U5 (LF) $/ \mathrm{U}$ U3(I)E)/U/Th $\mathrm{Pu} / \mathrm{Ts}$

INIR-US(NAT)/U US(SFill) $/ \mathrm{U}$

IIS(DE:/U/Th $\mathrm{U} 3(\mathrm{DE}): \mathrm{U} / \mathrm{T} \mathrm{T}_{1}$ $\mathrm{Pu} / \mathrm{U}$

$\mathrm{Pu} / \mathrm{Th}$

ITTR-U5 (IIE)/U-'T U5 (I.E) $/ U$ US (DE) $/ \mathrm{UI} / \mathrm{Th}$ U5 (IIE)/Th U3(UF) $/ U /$ 'Th U3/Ths

- Mu/lits

FBR-Pu-U/U

$\mid \mathrm{nu}-\mathrm{l} / \mathrm{Tl}$

Fossil

Nuclcar
1439 
Table F.11

CAPITAL CONSTRUCTION CASII FLOWS FOR

ONCl:-TIROU(A) (Throwaway)

NLL REACIOR CASE--SMALL URANIUM SUPPLY

(Millions of $\$ /$ Year)

\begin{tabular}{|c|c|c|c|c|c|c|c|c|c|}
\hline \multirow[b]{2}{*}{ Year } & \multirow[b]{2}{*}{$\begin{array}{l}\text { Fossil } \\
\text { Plants }\end{array}$} & \multirow[b]{2}{*}{$\begin{array}{r}\text { Nuclear } \\
\text { Plants } \\
\end{array}$} & \multicolumn{4}{|c|}{ 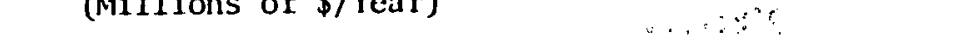 } & & \multirow[b]{2}{*}{$\begin{array}{r}U_{3}{ }^{O_{8}} \\
\text { Mines } \\
\end{array}$} & \multirow[b]{2}{*}{$\begin{array}{r}\mathrm{InO}_{2} \\
\text { Mines } \\
\end{array}$} \\
\hline & & & $\begin{array}{c}\text { Fabrication } \\
\text { Plants } \\
\end{array}$ & Rep & $\begin{array}{l}\text { rocessing* } \\
\text { Plants } \\
\end{array}$ & & $\begin{array}{l}\text { Enrichinent } \\
\text { Plants } \\
\end{array}$ & & \\
\hline 1970 & 5,380 & 3,450 & 27 & & - & & 133 & 213 & - \\
\hline 1975 & 8,860 & 5,210 & 29 & .. & - & & 190 & 296 & - \\
\hline 1980 & 7,270 & 9,000 & 72 & & - & & 354 & 554 & - \\
\hline 1985 & 9,330 & 9,290 & 45 & & - & & 346 & 511 & - \\
\hline 1990 & 7,330 & 11,520 & 65 & & 240 & & 420 & 612 & - \\
\hline 1995 & 14,470 & 10,440 & 27 & & 616 & & 282 & 421 & - \\
\hline 2000 & 19,910 & 8,780 & 48 & & 269 & & 447 & 370 & - \\
\hline 2005 & 27,720 & 5,210 & 19 & & 213 & & 109 & 201 & - \\
\hline 2010 & 36,820 & 3,060 & 43 & & 141 & & 106 & 194 & $-\cdot$ \\
\hline 2015 & 39,910 & 1,980 & 14 & & 48 & & 62 & 100 & - \\
\hline 2020 & 44,910 & 2,040 & 15 & $\therefore$ & 24 & & 97 & 133 & - \\
\hline 2025 & 49,850 & 2,420 & 10 & & 177 & & 50 & 60 & - \\
\hline 2030 & 56,960 & 2,640 & 13 & & 120 & & 69 & 104 & - \\
\hline 2035 & 61,230 & 2,880 & 12 & . & 72 & & 43 & $89^{\prime}$ & $\therefore$ \\
\hline 2040 & 70,290 & 2,460 . & 20 & & 72 & & 85 & 172 & - \\
\hline
\end{tabular}

Reprocessing Plants in the throwaway options represent the facilities required for disposal of all spent fuel discharged. 
Table F.12

CAPITAL. CONSTRUCTION CASII FLCIWS

IOR CASI: U-233 iNI) U-235 RF:CYCLF- - ALL REACTOR

CASER --SMALI. UIRANIUM SUPPLY

iMillions of $\$ /$ Year)

\begin{tabular}{|c|c|c|c|c|c|c|c|}
\hline$\underline{\text { Year }}$ & $\begin{array}{l}\text { I:ossil } \\
\text { Plants } \\
\end{array}$ & $\begin{array}{r}\text { Nuclear } \\
\text { Plants } \\
\end{array}$ & $\begin{array}{c}\text { Fabrication } \\
\text { plants } \\
\end{array}$ & $\begin{array}{l}\text { Reprocessing } \\
\text { Plants } \\
\end{array}$ & $\begin{array}{c}\text { Enriclunent } \\
\text { Plants } \\
\end{array}$ & $\begin{array}{r}\mathrm{U}_{3} \mathrm{O}_{8} \\
\text { Mines } \\
\end{array}$ & $\begin{array}{c}\mathrm{ThO}_{2} \\
\text { Mines } \\
\end{array}$ \\
\hline 1970 & 5,380 & 3,450 & 27 & : & 133 & 213 & - \\
\hline 1975 & 8,870 & 5,130 & 29 & $\therefore$ & 189 & 292 & - \\
\hline 1980 & 7,820 & 8,640 & 66 & - & 321 & 502 & - \\
\hline 1985 & 7,440 & 9,430 & 92 & - & 384 & 552 & 2 \\
\hline 1990 & 10,960 & 9,070 & 61 & 525 & 467 & 233 & 8 \\
\hline 1995 & 13,190 & 11,580 & 75 & 139 & 453 & 636 & 12 \\
\hline 2000 & 14,800 & 18,180 & 175 & 218 & 555 & 455 & 14 \\
\hline 2005 & $13,76 \mathrm{~J}$ & $\approx 0,170$ & 338 & 336 & 431 & 472 & 10 \\
\hline 2010 & 26,451 & 15,680 & 167 & 211 & 305 & 326 & 7 \\
\hline 2015 & 27,860 & 15,570 & 169 & 195 & 327 & 418 & 8. \\
\hline 2020 & 36,620 & 10,870 & 132 & 387 & 189 & 21 & 6 \\
\hline 2025 & 44,690 & 7,530 & 72 & 1.91 & 0 & 21 & 4 \\
\hline 2030 & 53,900 & 6,170 & 132 & 185 & 67 & 0 & 7 \\
\hline 2035 & 59,530 & 4,330 & 179 & 227 & 0 & 44 & 1 \\
\hline 2040 & 70,290 & 2,550 & 17 & 5 & 39 & 117 & 0 \\
\hline
\end{tabular}


Table F.13

CAPITAL CONSTRUCTION CASH FLOWS

FOR RESTRICTIS RECYCLE - - ALL REACTOR CASE - -

$$
\text { SMALL URANIUM SUPPLY }
$$

(Millions of $\$ /$ Year)

\begin{tabular}{|c|c|c|c|c|c|c|c|}
\hline Year & $\begin{array}{l}\text { Possil } \\
\text { Píants } \\
\end{array}$ & $\begin{array}{r}\text { Nuclear } \\
\text { Plants } \\
\end{array}$ & $\begin{array}{c}\text { Fabrication } \\
\text { Plants } \\
\end{array}$ & $\begin{array}{c}\text { Reprocessing } \\
\text { Plants } \\
\end{array}$ & $\begin{array}{l}\text { Enrichnent } \\
\text { Plants } \\
\end{array}$ & $\begin{array}{r}U_{3}{ }{ }_{8} \\
\text { Mines } \\
\end{array}$ & $\begin{array}{r}\mathrm{MhO}_{2} \\
\text { Mines } \\
\end{array}$ \\
\hline 1970 & 5,380 & 3,450 & 27 & - & 133 & 213 & - \\
\hline 1975 & 8,370 & 5,130 & 29 & - & 189 & 292 & - \\
\hline 1980 & 7,820 & 9,020 & $66^{\circ}$ & - & 321 & 502 & - \\
\hline 1985 & 3,943 & 13,870 & 134 & - & 531 & 781 & 2 \\
\hline 1990 & 4,600 & 15,140 & 135 & 642 & 424 & 359 & 6 \\
\hline 1995 & $B, 730$ & 17,760 & 159 & 514 & 341 & 541 & 8 \\
\hline 2000 & 8,200 & 24,570 & 252 & 525 & 511 & 742 & 7 \\
\hline 2005 & $.7,950$ & 30,000 & 453 & 592 & 439 & 608 & 16 \\
\hline 2010 & 9,300 & 37,110 & 813 & 714 & 292 & 348 & 30 \\
\hline 2015 & 15,110 & 32,530 & 622 & 788 & 277 & 354 & 23 \\
\hline 2020 & 17,410 & 35,490 & 750 & 834 & 233 & $134^{\circ}$ & 27 \\
\hline 2025 & 21,690 & 37,030 & 798 & 787 & 93 & 146 & 24 \\
\hline 2030 & 27,110 & 36,720 & 516 & $644:$ & $1: 17$ & 133 & 14 \\
\hline 2035 & 36,110 & 31,750 & 531 & 630 & 48 & 114 & 16 \\
\hline 2040 & 48,620 & 26,870 & 575 & 498 & 26 & 57 & 18 \\
\hline
\end{tabular}


Table F.14

CAPITAL CONSTRUCTION CASH FLOWS

FOR FULL RECYCLE--ALL REACTOR CASE - SMALL URANIUM SUPPLY (Millions of $\$ /$ Year)

\begin{tabular}{|c|c|c|c|c|c|c|c|c|c|}
\hline Year & $\begin{array}{l}\text { Fossil } \\
\text { Plants } \\
\end{array}$ & $\begin{array}{r}\text { Nuclear } \\
\text { Plants } \\
\end{array}$ & & $\begin{array}{c}\text { Fabricaticn } \\
\text { Plants } \\
\end{array}$ & $\begin{array}{c}\text { Reprocessing } \\
\text { Plants } \\
\end{array}$ & & $\begin{array}{l}\text { Enrichinent } \\
\text { Plants } \\
\end{array}$ & $\begin{array}{r}u_{3} O_{8} \\
\text { Mines } \\
\end{array}$ & $\begin{array}{r}\mathrm{ThO}_{2} \\
\text { Mines } \\
\end{array}$ \\
\hline 1970 & 5,380 & 3,450 & & 27 & - & & 133 & 213 & - \\
\hline 1975 & 8,870 & 5,130 & & 29 & - & & 189 & 292 & - \\
\hline 1980 & 7,820 & 9,000 & & 66 & - & & 321 & 502 & - \\
\hline 1985 & 4,090 & $1.3,700$ & & 128 & - & & 514 & 763 & - \\
\hline 1990 & 5,970 & 13,500 & & 106 & 640 & & 395 & 318 & 1 \\
\hline 1995 & 8,470 & 18,570 & . & 174 & 416 & & 372 & 59.5 & 2 \\
\hline 2000 & 8,640 & 24,310 & & 216 & 507. & & 619 & 653 & 11 \\
\hline 2005 & 7,890 & 30,920 & & 467 & 518 & & 471 & 483 & 15 \\
\hline 2010 & 8,870 & 37,300 & & 8.20 & 665 & & 304 & 319 & 15 \\
\hline 2015 & 15,140 & 32,850 & & 489 & 768 & & 490. & 511 & 17 \\
\hline 2020 & $17,4.10$ & $3.5,580$ & & 654 & 822 & & 388 & 203 & 23 \\
\hline 2025 & 21,560 & 33,200 & & 531 & 697 & & 190 & 279 & 21 \\
\hline 2030 & 27,900 & 35,160 & $\therefore \quad 3$ & 551 & 734 & & 218 & 168 & 24 \\
\hline 2035 & 37,750 & 29,760 & & 786 & $734 \therefore$ & 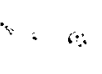 & 0 & 4 & 23 \\
\hline 2040 & 49,930 & 25,130 & & 670 & 615 & & 0 & 45 & 16 \\
\hline
\end{tabular}


Table F.15

SUMMARY OF THORILM ASSESSUENT PROGRAM. STUDY RESULTS LARG̣E $\mathrm{U}_{3} \mathrm{O}_{8}$ SUPPLY

System Costs ( $\$ B)$ 1977 through 2050 Discounted at

$$
\begin{aligned}
& 41 / 2 \% \\
& 71 / 2 \% \\
& 10 \%
\end{aligned}
$$

Fuel Cycle Options

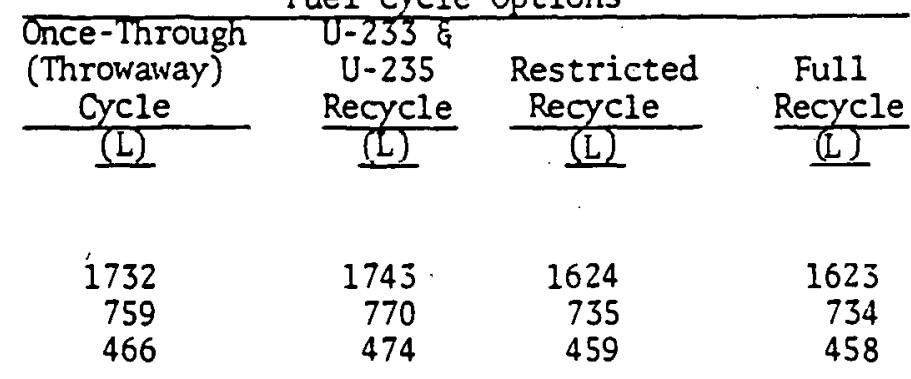

Levelized System

Power Costs in 2025 (mills/kwhr)

18.8

18.5

17.2

17.3

Cumulative Capacity Built (Give) through 2050

Nuclear

$1257(24 \%)$

$1815(34 \%) 3296(62 \%)$

$3415(64 \%)$

Fossil

Annual Coal Consump-

tion in 2025 ( $10^{6}$ tons)

4130

3410

1820

1920

Cumulative $\mathrm{U}_{3} \mathrm{O}_{8}$ Con-

sumption ( 106 tons)

through

$$
2026
$$

2050

4.75
6.10

4.41

5.75

4.48

4.43

5.75

6.29

Maximum Annual Enrich-

ment Requirement

through 2050 (106 siv)

yr)

93

119

101

93

$\mathrm{U}_{3} \mathrm{O}_{8}$ Utilization $(1)$

(ton $\mathrm{U}_{3} \mathrm{O}_{8} / \mathrm{Gie}$ )

4994

3271

1782

2022

Enrichment Utilization (2)

(10b Siv/Gie)

3.02

2.63

1.25

1.16

(1) Qmulative $U_{3} O_{8}$ consumed through 2050 (including forvard committment) per cumlative nuclear capacity built through 2050

(2) Cumulative enrichment requirements through 2050 per cumulative nuclear capacity built through 2050 
Table F.16

SUMMARY OF THORIUM ASSESSIENT PROGRAM STUDY RESULTS LARGE $\mathrm{U}_{5} \mathrm{O}_{8}$ SUPPLY

System Costs ( $\$ B$ )

\begin{tabular}{|c|c|c|c|}
\hline \multicolumn{4}{|c|}{0} \\
\hline $\begin{array}{c}\text { Once-Ihrough } \\
\text { (Throwaway) } \\
\text { Cycle } \\
\end{array}$ & $\begin{array}{l}\text { U-25J } \\
\text { Recycle }\end{array}$ & $\begin{array}{l}\text { Restricted } \\
\text { Recycle }\end{array}$ & $\begin{array}{c}\text { Full } \\
\text { Recycle }\end{array}$ \\
\hline (S) & (S) & (S) & . \\
\hline $\begin{array}{r}1738 \\
762 \\
467\end{array}$ & $\begin{array}{r}1730 \\
768 \\
473\end{array}$ & $\begin{array}{r}1608 \\
731 \\
457\end{array}$ & $\begin{array}{l}1612 \\
731 \\
458\end{array}$ \\
\hline
\end{tabular}

Levelized System

Power Costs in

2025 (mills/kwhr)

18.8

18.3

16.7

16.9

Cumulative Capacity

Built (Give) through

2050

Nuclear

$1335(25 \%)$

$2136(40 \%) \quad 3844(72 \%) \quad . \quad 3580(67 \%)$

Fossil

$3981(75 \%)$

$3180(60 \%) \quad 1472(28 \%) \quad: 1736(33 \%)$

Annual Coal Consump-

tion in 2025 (106 tons)

4050

3050

1490

1660

Cumulative $\mathrm{U}_{3} 0_{8}$ Cons"

sumption ( 106 tons)

through

$$
2026
$$

2050

4.61
0.12

4.41

4.15

4.20

5.75

5.81

6.34

Maximm Annual Enrich-

ment Requirement

through 2050 (106 siu/

$y$ )

81

121

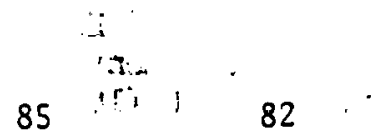

$\mathrm{U}_{3} \mathrm{C}_{3}$ Utilization $(1)$

(t: $n U^{3} \mathrm{O}_{8} /$ GWe)

4801

2856

1572

1964

Enrichment Utilization (2)

(106 SW/Give)

2.64

2.24

i. 05

1.07

(1) Cumulative $\mathrm{U}_{7} \mathrm{O}_{8}$ consuned through 2050 (including forward committment) per cumulative nưclear capacity built through 2050

(2) Cumulative enrichment requirements through 2050 per cumulative nuclear capacity built through 2050 
Table F.17

SUMMARY OF THORIUM ASSESSMENT PROGRAM STUDY RESULTS LARTE $\mathrm{U}_{3} \mathrm{O}_{8}$ SUPPLY

System Costs (\$B)

1977 through 2050

Discounted at

$$
\begin{aligned}
& 41 / 2 \% \\
& 71 \% \\
& 10 \%
\end{aligned}
$$

Levelized System

Power Costs in

2025 (mills/kwhr)

Cumulative Capacity

Built (GNe) through

2050

\section{Nuclear \\ Fossil}

Annual Coal Consump:

tion in 2025 ( $10^{6}$ tons)

Cumulative $\mathrm{U}_{3} \mathrm{O}_{8}$ Con-

sumption (106 tons)

through

$$
2026
$$

2050

Once-Through
$\begin{aligned} & \text { (Throwaway) } \\ & \text { Cycle }\end{aligned}$
(H)

Fuel Cycle Options

U-233 \&

U-235

Recycle Recycle

(H) (H)

Full

Recycle

(H)

$$
\begin{array}{r}
1747 \\
765 \\
468
\end{array}
$$

$$
\begin{array}{r}
1749 \\
766 \\
470
\end{array}
$$

$$
\begin{array}{r}
1662 \\
746 \\
463
\end{array}
$$

18.8

$1058(20 \%)$

$4258(80 \%)$

4420

$1444(27 \%)$
$3872(73 \%)$

$4238(80 \%)$

$1078(20 \%)$

3780

1830

1740

Maximm Annual Enrichment Requirement through 2050 (106 SWU/ yT)

$\mathrm{U}^{-} \mathrm{O}_{8}$ Utilization ${ }^{(1)}$.

(Zon $\mathrm{U}_{3} \mathrm{O}_{8} / \mathrm{GWe}$ )

Enrichment Utilization (2)

(10? 5 Wu/Gie)
4.59

5.75

84

97

5652

\subsection{0}

4.35

.5 .75

4.43

5.25

4.45

5.29

(1) Cumilative $\mathrm{U}_{3} \mathrm{O}_{8}$ consumed through 2050 (including forward committment) per cumulative nuclear capacity built through 2050

(2) Cumulative enrichnent requirements through 2050 per cumulative nuclear capacity built through 2050 
Table F.18

SURMARY OF THORILM ASSESSTENT PROGRAM STUDY RESULTS LARGE $\mathrm{U}_{5} 0_{8}$ SUPPLY

System Costs ( $\$ B)$ 1977 through 2050

Discounted at

$41 / 2 \%$

$71 / 2 \%$

$10 \%$

Levelized System

Power Costs in

2025 (mills/kwhr)

Cumulative Capacity

Built (Give) through

2050

Nuclear

Fossil

Annual Coal Consuntp-

tion in 2025 (106 tons)

Cumulative $\mathrm{U}_{3} \mathrm{O}_{8}$ Con-

sumption (106 tons)

through

2026

2050

Maximm Annual Enrich-

ment Requirement

through $2050\left(10^{6}\right.$ sitU/

$y r$ )

$\mathrm{U}_{3} \mathrm{O}_{8}$ Utilization $(1)$

(ton $\mathrm{U}_{3} \mathrm{O}_{8} / \mathrm{Give}$ )

Enrichment Utilization (2)

(10 $0^{6} \mathrm{Sin} / \mathrm{GWe}$ )
Fuel Cycle Options

\begin{tabular}{|c|c|c|c|}
\hline $\begin{array}{l}\text { Once-Through } \\
\text { (Throwaway) } \\
\text { Cycle }\end{array}$ & $\begin{array}{c}U-233 \& \\
U-235 \\
\text { Recycle }\end{array}$ & $\begin{array}{l}\text { Restricted } \\
\text { Recycle }\end{array}$ & $\begin{array}{l}\text { Full } \\
\text { Recycle }\end{array}$ \\
\hline (G) & $\frac{(G)}{.}$ & (G) & \\
\hline $\begin{array}{r}1745 \\
764 \\
468\end{array}$ & $\begin{array}{r}1724 \\
762 \\
469\end{array}$ & $\begin{array}{r}1619 \\
735 \\
460\end{array}$ & $\begin{array}{r}1611 \\
\because 4733 \\
458\end{array}$ \\
\hline
\end{tabular}

18.8

18.3

16.7

16.8

$1211(23 \%)$

$2143(40 \%) \quad 3517(66 \%)$

$3811(72 \%)$

$4105(77 \%) \quad 3173(60 \%) \quad 1799(34 \%) \div 1505(28 \%)$

4320

2880

1520

1480

$\begin{array}{llll}4.56 & 4.43 & 4.46 & 4.21 \\ 5.81 & 6.39 & 5.75 & 6.17\end{array}$

6.17

4.56
5.81

86

123

101

101

5006

3099

$1715^{i}$

1721

3.02

2.46

1.17

1.33

(1) Cumlative $\mathrm{U}_{3} \mathrm{O}_{8}$ consumed through 2050 (including forward committment) per cumlative nứclear capacity built through 2050

(2) Cumulative enrichment requirements through 2050 per cumulative nuclear capacity built through 2050 
Table F.19

SUMMARY OF THORILM ASSESSMENT PROGRAM STUDY RESULTS LARGE $\mathrm{U}_{3} \mathrm{O}_{8}$ SUPPLY

System Costs ( $\$ B)$

1977 through 2050

Discounted at

$4 \cdot 1 / 2 \%$

$71 / 28$

$10 \%$

\begin{tabular}{|c|c|c|c|}
\hline $\begin{array}{l}\text { Once-Through } \\
\text { (Throwaway) } \\
\text { Cycle } \\
\text { (A) }\end{array}$ & $\begin{array}{c}\mathrm{U}-253 \text { \& } \\
\mathrm{U}-235 \\
\text { Recycle } \\
\frac{\text { (A) }}{}\end{array}$ & $\begin{array}{c}\begin{array}{c}\text { Restricted } \\
\text { Recycle }\end{array} \\
\text { (A) }\end{array}$ & $\begin{array}{c}\text { Full } \\
\text { Recycle }\end{array}$ \\
\hline $\begin{array}{r}1731 \\
759 . \\
465\end{array}$ & $\begin{array}{r}1717 \\
763 \\
471\end{array}$ & $\begin{array}{r}1602 \\
728 \\
456\end{array}$ & $\begin{array}{r}1602 \\
729 \\
456\end{array}$ \\
\hline
\end{tabular}

Levelized System

Power Costs in

2025 (mills/kwhr)

Cumulative Capacity

Built (GNe) through

2050

Nuclear

Fossil

1.5 .0

18.2

16.6

16.7

Annual Coal Consump-

tion in 2025 ( $10^{6}$ tons)

$1339(25 \%)$

$3977(75 \%)$

$2263(43 \%)$

$3053(57 \%)$

$3680(69 \%)$

$1636(31 \%)$

$3725(70 \%)$

4030

2610

1490

1460

Cumulative $\mathrm{U}_{3} \mathrm{O}_{8}$ Con-

sumption (106 tons)

through

2026
2050

\subsection{4}

6.23

4.43

4.18
5.92

4.07

6.18

5.92

6.22

Maximm Annual Enrich-

ment Requirement

through 2050 ( $10^{6}$ SIIU/

yr)

$\mathrm{U}_{3} \mathrm{O}_{8}$ Utilization $(1)$

( $\tan \mathrm{U}_{3} \mathrm{O}_{8} / \mathrm{GNe}$ )

1)

Enrichment Utilization (2)

(10 5 SiU/GWe)

89

4864

2.82
125

2838

1666

2.37

1.12

1.36

(1) Cumulative $\mathrm{U}_{3} \mathrm{O}_{8}$ consumed through 2050 (including forward committment) per cumulative nưclear capacity built through 2050

(2) Cumulative enrichment requirements through 2050 per cumulative nuclear capacity built through 2050 
Table F.20

TIORIIN ASSI:SSAIINT PROCRAM RESULTS

l.arge $\mathrm{U}_{3} \mathrm{OS}$ Supply

INSTAAIIED CAPACITY (GWe) in YEAR 2026/LLVI:LIZI:D POIVER COSTS IMIIls;Kwhr) in YEAR 2025

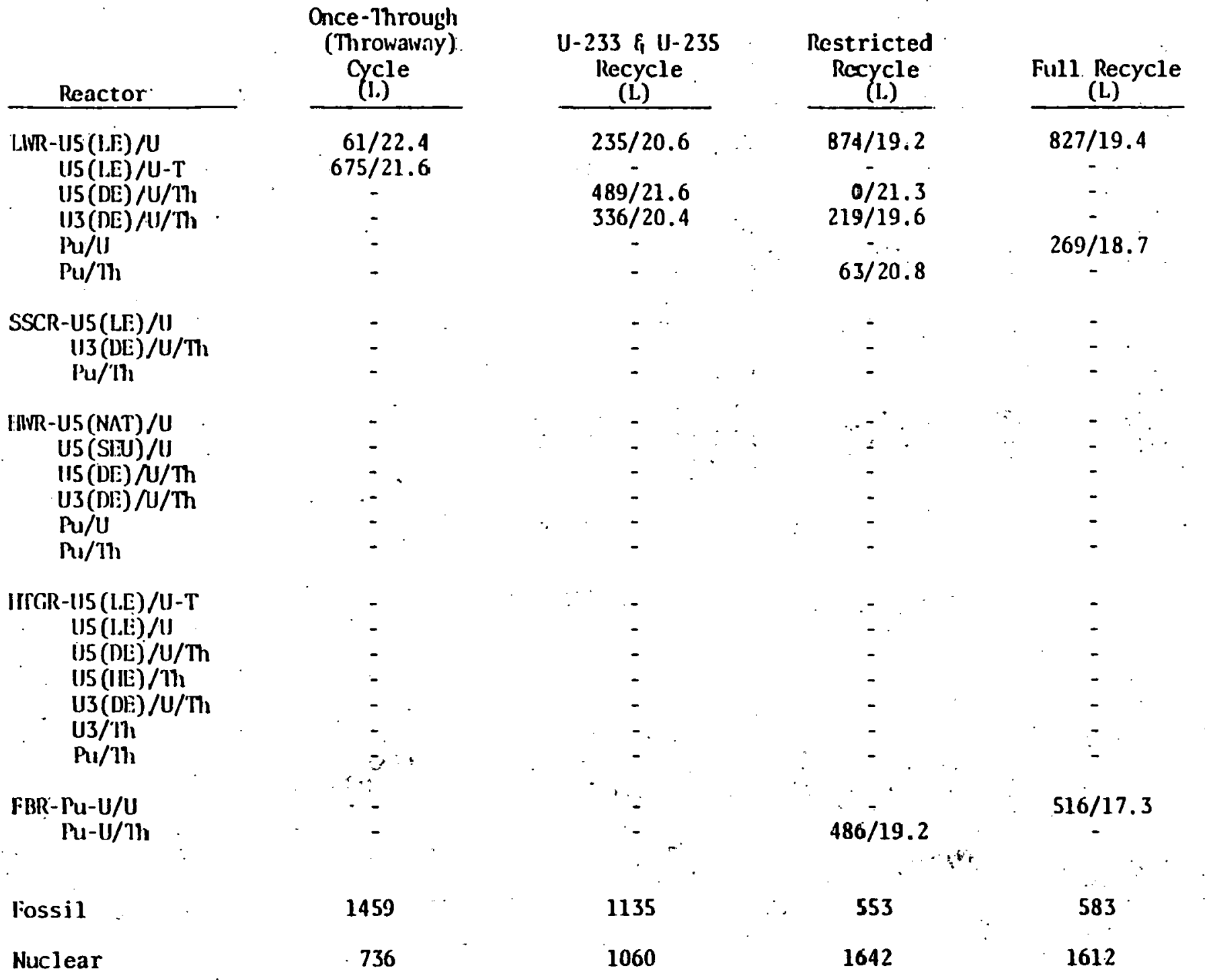


Table F. 21

TIORIIM ASST:SSMENT PROGIRM RESULTS

Large $\mathrm{U}_{3} \mathrm{O}_{8}$ Supply

INSTALIED CAPACITY (GVe) in YTAR 2026/LEVELIZED POWT:R COSTS (Mills/Kwhr) in YTEAR 2025

\begin{tabular}{|c|c|c|c|c|}
\hline Reactor & $\begin{array}{c}\text { Once-Through } \\
\text { (Throwaway) } \\
\text { Cycle } \\
(\mathrm{S}) \\
\end{array}$ & $\begin{array}{c}\text { U-233 \& U-235 } \\
\text { Recycle } \\
\text { (S) }\end{array}$ & $\begin{array}{c}\text { Restricted } \\
\text { Recycle } \\
\text { (S) }\end{array}$ & $\begin{array}{l}\text { Full Recycle } \\
\text { (S) }\end{array}$ \\
\hline $\begin{array}{l}\text { LIVR-US (LE)/U: } \\
\text { US (LE)/U-T. }\end{array}$ & $205 / 22.3$ & $128 / 21.0$ & $298 / 19.2$ & $283 / 19.5$ \\
\hline U5 (DF) $/ U / 77$ & - & - & - & - \\
\hline $\mathrm{U} 3(\mathrm{DE}) / \mathrm{U} / \mathrm{Th}$ & - & - & - & - \\
\hline$R_{1} / \mathbf{U}$ & - & $525 / 21.4$ & $0 / 21.1$ & $344 / 18.1$ \\
\hline $\mathrm{Pu} / \mathrm{Th}$ & - & - & - & - \\
\hline SSCR-US(LE) /U & $568 / 21.2$ & $\begin{array}{r}29 / 20.4 \\
506 / 20.1\end{array}$ & $650 / 18.2$ & $603 / 18.4$ \\
\hline $\begin{array}{l}. . \mathrm{U}(\mathrm{Dr}) / \mathrm{U} / \mathrm{Th} \\
\mathrm{Pu} / \mathrm{Th}\end{array}$ & - & $\frac{500 / 20.1}{-}$ & $114 / 19.1$ & - \\
\hline IIIVR-U5(NAT)/U & - & - & - & - \\
\hline U5 (SLU)/U & - & - & - & - \\
\hline $115(\mathrm{DI}) / \mathrm{U} / \mathrm{Th}$ & - & - & - & - \\
\hline . $\quad 1 / 3(\mathrm{Dr}:) / \mathrm{II} / \mathrm{Th}$ & - & - & - & - \\
\hline $\mathrm{Mu} / \mathrm{U}$ & - & - & $=$ & - \\
\hline $\mathrm{Pu} / \mathrm{Th}$ & - & - & - & - \\
\hline HIGR-U5 (LE)/U-T & - & - & - & - \\
\hline U5(LE)/U & - & - & - & - \\
\hline $\mathrm{US}$ (DE)/U/Th & $-\therefore$ & - & - & - \\
\hline U5 (IIE)/1h & - & - & - & $\cdot$ \\
\hline $\mathrm{U} 3(\mathrm{DE}) / \mathrm{U} / \mathrm{Th}$ & - & - & - & - \\
\hline - U3/Th & $\therefore \therefore-$ & - & - & - \\
\hline$M_{1} / 11$ & 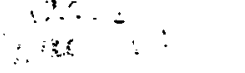 & - & : & - \\
\hline $\mathrm{FBR}-\mathrm{Pu}-\mathrm{U} / \mathrm{U}$ & $\therefore-\cdots$ & - & 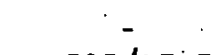 & $475 / 17.5$ \\
\hline $\mathrm{Pu}-\mathrm{U} / \mathrm{Th}$ & $: \quad-$ & - & $385 / 17.7$ & - \\
\hline lossil & 1422 & $1007^{\circ}$ & 442 & 490 \\
\hline Nuclear & 773 & 1188 & 1753 & 1705 \\
\hline
\end{tabular}


Table F. 22

TTDRIUM ASSESSAENT PROGRAM RESULTS

$$
\text { Large } \mathrm{U}_{3} \mathbf{0}_{8} \text { Supply }
$$

INSTALLEI) CAPACITY (GWe) in YTAR 2026/LEVELLI2ED POIER COSTS (Mi11s/Kwhr) in YEAR 2025

\begin{tabular}{|c|c|c|c|c|}
\hline Reactor & $\begin{array}{l}\text { Once-Throigh } \\
\text { (Throwawey) } \\
\text { Cycle } \\
\text { (II) } \\
\end{array}$ & $\begin{array}{c}\mathrm{U}-233 \text { \& U U-235 } \\
\text { Recycle } \\
(\mathrm{H}) \\
\end{array}$ & $\begin{array}{c}\text { Restricted } \\
\text { Recycle } \\
\text { (Ii) }\end{array}$ & $\begin{array}{l}\text { Full Recycle } \\
(\mathrm{H})\end{array}$ \\
\hline LIVR-US (I.E)/U & $617 / 21.8$ & $883 / 21.0$ & $785 / 19.2$ & $792 / 19.3$ \\
\hline U5 (LI:)/U-T & - & - & - & - \\
\hline U5 (DF) $/ U / T h$ & - & - & - & .- \\
\hline U3 (DE) $/ U / \mathrm{Th}$ & - & - & - & - \\
\hline $\mathrm{Ru} / \mathrm{U}$ & - & - & - & - \\
\hline $\mathrm{Pu} / \mathrm{Th}$ & - & - & - & - \\
\hline SSCR-U5 (IJ)/U & - & - & - & - \\
\hline 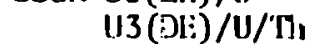 & - & - & - & - \\
\hline $\mathrm{Pu} / \mathrm{Th}$ & - & - & - & - \\
\hline InUR-U5(NAT)/U & $0 / 24 . \epsilon$ & $0 / 26.8$ & $0 / 23.8$ & $0 / 23.8$ \\
\hline US (SEU) $/ U$ & $0 / 21 . \varepsilon$ & $0 / 22.9$ & $0 / 21.1$ & $0 / 21.1$ \\
\hline 115 (DE) $/ U / \mathrm{Th}$ & - & $0 / 26.8$ & $0 / 28.8$ & - \\
\hline U3 (DA:)/U/ $/ \prod_{1}$ & - & $0 / 20.6$ & $158 / 17.8$ & - \\
\hline $\mathrm{Pu} / \mathrm{U}$ & - & - & - & $0 / 21.1$ \\
\hline $\mathrm{Pu} / \mathrm{Th}$ & - & - & $0 / 22.2$ & - \\
\hline IrTCR-US(LE)/U-T & - & - & - & - \\
\hline US (LE)/U & - & - & - & - \\
\hline $\mathrm{U} 5(\mathrm{DE}) / \mathrm{U} / \mathrm{Th}$ & - & - & - & - \\
\hline U5 (IIE)/Th & - & - & - & - \\
\hline U3 (DE: $/ U / \Gamma h$ & - & - & - & - \\
\hline U3/Th & - & - & - & - \\
\hline $\mathrm{Pu} / \mathrm{Th}$ & - & - & - & - \\
\hline $\mathrm{IFBR}-\mathrm{Pu}-\mathrm{U} / \mathrm{U}$ & - & $\therefore$ & $\therefore$ & $886 / 17.6$ \\
\hline $\mid m a-L / / I h$ & - & - & $7.34 / 18.2$ & - \\
\hline & & & & \\
\hline liossil & 1578 & 1312 & 548 & 517 \\
\hline Nuclear & 617 & 883 & 1647 & 1678 \\
\hline
\end{tabular}


Table F.23

TIORIUM ASSESSAENT PBOGRAM RESUITS

Large $\mathrm{U}_{3} \mathbf{0}_{8}$ Supply

INSTALLED CAPACITY (GWe) in YTAR 2026/LEVELIZED POINER COSTS (Mli1ls/Kwhr) in YEAR 2025

$$
\text { Once-Through }
$$

\begin{tabular}{l} 
Reactor \\
\hline LIR-US(LE) $/ U$ \\
US(LE) $/ U-T$ \\
U5(DE) $/ \mathrm{U} / \mathrm{Th}$ \\
U3(DE) $/ \mathrm{U} / \mathrm{Th}$ \\
$\mathrm{Pu} / \mathrm{U}$ \\
$\mathrm{Pu} / \mathrm{7h}$
\end{tabular}

(Throwalvay)

$\operatorname{SSCR}-\mathrm{U} 5(\mathrm{LF}) / \mathrm{U}$

$\mathrm{U} 3(\mathrm{DF}) / \mathrm{U} / \mathrm{Th}$

$\mathrm{Pu} / \mathrm{Th}$

I IVR-U5(NAT) /U

U5 (SEU) $/ U$

IIS (DF:) $/ \mathrm{U} / \mathrm{Th}$

$\mathrm{U} 3(\mathrm{DE}) / \mathrm{W} / \mathrm{Th}$

$\mathrm{Pu} / \mathrm{U}$

$\mathrm{Pu} / \mathrm{Th}$

IIRTR-US(LE)/U-T

U5 (LE)/U

US (DF) $/ U / \mathrm{Th}$

US (IIE)/Th

U3(DF) $/ U / T h$

U3/Th

Pu/'Ih
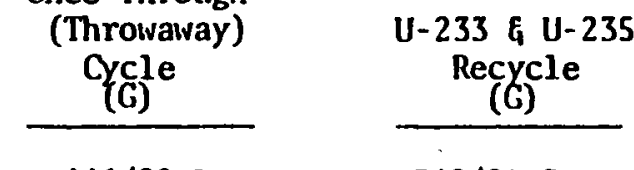

$446 / 22.1$

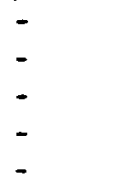

$342 / 21.5$

$-$

$-P u-U / U$

$\mathrm{Pu}-\mathrm{U} / \mathrm{Th}$

$218 / 21.4$

$0 / 20.8$

$723 / 19.9$

$179 / 19.7$

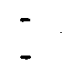

$-$

Fossil

1531

951

664

1244

$0 / 19.3$

$0 / 18.9$

Restricted (G)

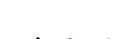

$-$

-

Full Recycle

(G)

Nuclear

$474 / 19.5$

-

$-$

-
$27 / 18.2$

\section{$275 / 17.7$}

$275 / 17.7$

$168 / 17.7$

$363 / 17.1$

$554 / 19.1$

478

435

1760 
nuclear market penetration by about 200 Give (mostly with converter reactors) in the year 2025 over the LiVR strategy except in the throwaway option where the SSCR available was not optinized for throwaway.

The CANDU reactors made available in the $H$ reactor strategy (Table F.8) did very poorly in competing with LWR's with and without FBR's. The only fuel cycle option in which CANDU's were built was the restricted recycle option where the only U-233 burner was a CANDU design.

The installed capacity of HIGR's shown in Table F.9 indicates. their ability to compete when operating on the $\mathrm{T} / \mathrm{U}-233$ fuel cycle: The HTGR optinized for throwaway and fueled with low-enriched U-235 did not compete nearly as well as the LWR optinized for throwaway. However, with the U-233 and U-235 recycle option, the denatured U-235 and U-233 burning HTGR's comprised $83 \%$ of the nuclear market and in the full recycle option the highly enriched HTGR's comprised $59 \%$ of the nuclear market. Note that in the restricted recycle option fissile Pu produced by primarily LWR's was recycled in FBR's whereas the U-233 was restricted to recycle in HTGR's.

If all reactors strategies are allowed to compete directly, the results are as shown in Table F.10. The SSCR and HTGR advanced converters as expected, win out over the LWR's with the CANOU's losing out completely due to high costs of heavy water inventories. The SSCR out-competes the LWR slightly in the throwaway option and dominates in the restricted cycle option. The HTGR, however, is the predominant converter design chosen in the U-233 and U-235 option and the full recycle option.

Similar results shown in Tables F. 20 through $F .28$ are obtained if a large $\mathrm{U}_{3} \mathrm{O}_{8}$ supply is assured. In general, all installed nuclear capacities are larger due to increased supply of low cost uranium ore. 
Table F.24

TIORIUM ASSESSHINT PROCRAM RESULTS

l.arge $\mathrm{U}_{3} \mathrm{O}_{8}$ Supply

INSTALLED CAPACITY (GWe) in YEAR 2026/LEVELIZED POIVER COSTS (Mills/Kwhr) in YEAR 2025

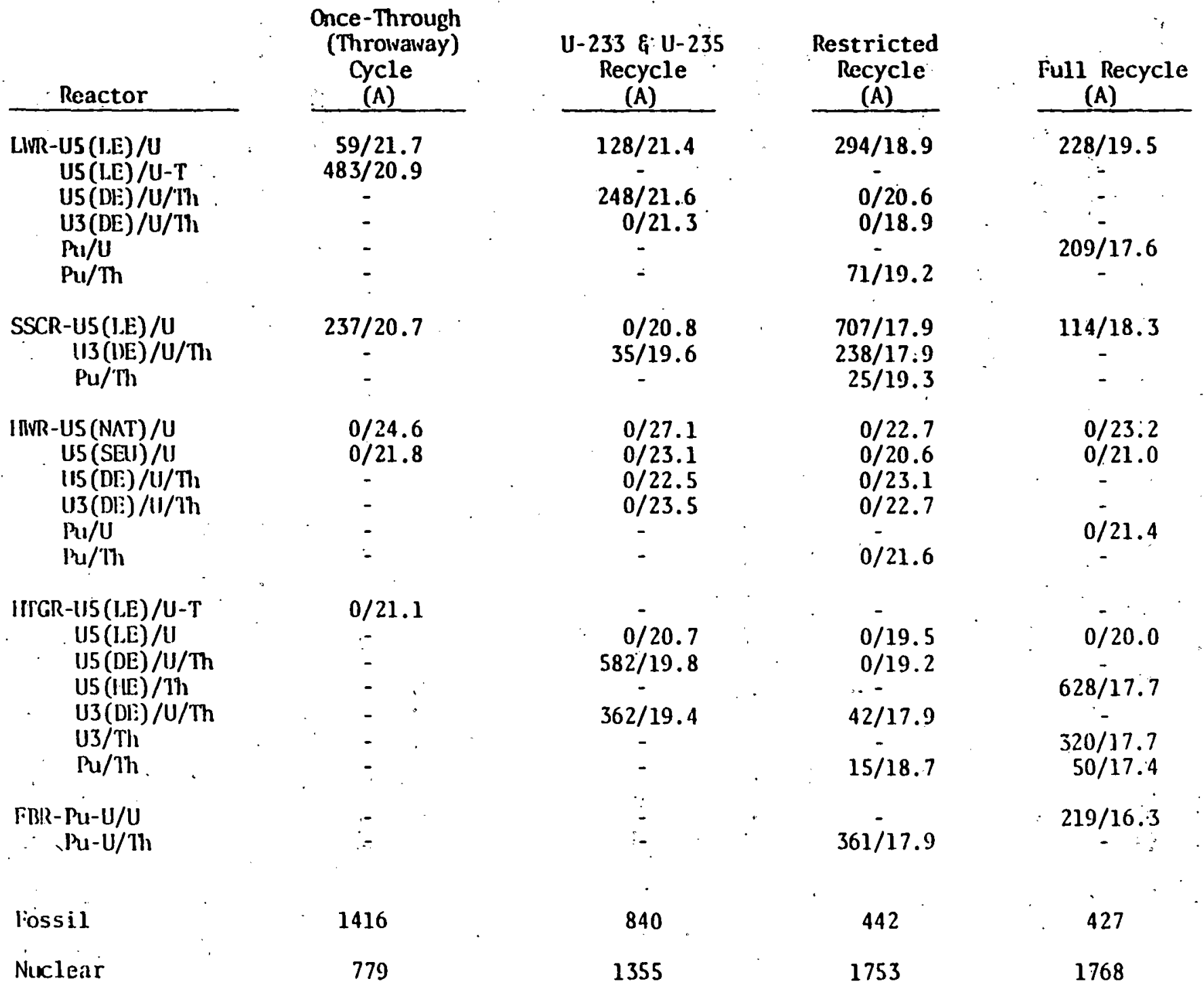


Table F.25

CAPITAL CONSTRUCTION CASH FLOIS

FOR ONCF:-TYMROIGI ('Throwaway) --ALL REACTOR CASE

large U Supply (Millions of $\$ /$ Year)

\begin{tabular}{|c|c|c|c|c|c|c|c|}
\hline Year. & $\begin{array}{l}\text { Fossil } \\
\text { plants } \\
\end{array}$ & $\begin{array}{l}\text { Nuclear } \\
\text { Plants } \\
\end{array}$ & $\begin{array}{l}\text { Fabrication } \\
\text { Plants } \\
\end{array}$ & $\begin{array}{l}\text { Reprocessing* } \\
\text { Plants } \\
\end{array}$ & $\begin{array}{c}\text { En:ichment } \\
\text { Plants } \\
\end{array}$ & $\begin{array}{r}\mathrm{U}_{3} \mathrm{O}_{8} \\
\text { Mines } \\
\end{array}$ & $\begin{array}{r}\mathrm{MnO}_{2} . \\
\text { Mines } \\
\end{array}$ \\
\hline 1970 & 5,380 & 3,450 & 27 & - & $\cdot 133$ & 213 & - \\
\hline 1975 & 8,860 & 5,210 & 29 & - & 190 & 296 & - \\
\hline 1980 & 7,270 & $\exists, 35]$ & $\pi 2$ & - & 534 & 554 & - \\
\hline 1985 & 6,470 & 12,170 & 80 & : & 469 & 712 & - \\
\hline 1990 & 4,360 & 15,280 & .7 & 632 & 560 & 824 & - \\
\hline 1995 & 7,370 & 18,330 & $? 6$ & 323 & ธَ70 & 956 & - \\
\hline 2000 & 10,810 & 18,750 & 90 & 328 & 525 & 894 & - \\
\hline 2005 & 17,960 & 15,070 & 50 & 362 & 100 & 608 & - \\
\hline 2010 & 26,600 & 14,130 & .98 & 168 & 375 & 630 & - \\
\hline 2015 & 31,600 & $9,8 \mathrm{G}_{1} 0$ & 48 & .256 & 253 & 458 & - \\
\hline 2020 & 40,750 & 5,810 & 39 & 402 & 151 & 244 & - \\
\hline 2025 & 48,310 & $3,46,0$ & 15 & 232 & 152 & 213 & $\therefore$ \\
\hline 2030 & 56,940 & 2,590 & 11 & 93 & 96 & 102 & - \\
\hline 2035 & 61,230 & 2,870 & 4 & 75 & 21 & 30 & - \\
\hline 2040 & 69,970 & $2,8 \subseteq 0$ & 31 & .96 & 79 & 168 & - \\
\hline
\end{tabular}

* Reprocessing plants in the throwalvay options represent the facilities required for disposal of all spent fuel discharged. 
Table F.26

CAPITAL CONSTRUICTION CASH FLOWS

FOR U-233 NND U-235 RI:CYCLF--ALL RI:ACTOR CASI:

Large U Supply

(Millions of $\$ /$ Year)

\begin{tabular}{|c|c|c|c|c|c|c|c|}
\hline Year & $\begin{array}{l}\text { Fossil } \\
\text { Plants }\end{array}$ & $\begin{array}{l}\text { Nuclear } \\
\text { Plants }\end{array}$ & $\begin{array}{l}\text { Fabrication } \\
\text { Plants } \\
\end{array}$ & $\begin{array}{c}\text { Reprocessing } \\
\text { Plants } \\
\end{array}$ & $\begin{array}{l}\text { Enrichment } \\
\text { Plants } \\
\end{array}$ & $\begin{array}{r}\mathrm{U}_{3} \mathrm{O}_{8} \\
\text { Mines } \\
\end{array}$ & $\begin{array}{c}\mathrm{ThO}_{2} \\
\text { Mines } \\
\end{array}$ \\
\hline 1970 & 5,380 & 3,450 & 27 & - & 133 & 213 & - \\
\hline 1975 & 8,870 & 5,130 & 29 & - & 187 & 292 & - \\
\hline 1980 & 7,820 & 9,020 & 66 & - & 321 & 502 & $\because$ \\
\hline 198.5 & 3,960 & 13,520 & 135 & - & 555 & 790 & 2 \\
\hline 1990 & 6,630 & 13,240 & 107 & 632 & 617 & 481 & 8 \\
\hline 1995 & $7,140 \div$ & 19,040 & 157 & 340 & 103 & 1,244 & 25 \\
\hline 2000 & $8,-420$ & 24,160 & 277 & 459 & 747 & 604 & 21 \\
\hline 2005 & 8,000 & 28,690 & 469 & 380 & 512 & 527 & 20 \\
\hline 2010 & 14,890 & 29,090 & 264 & 383 & 687 & 716 & 15 \\
\hline 2015 & 15,690 & 29,860 & 286 & 330 & 737 & 802 & 16 \\
\hline 2020 & 24,730 & 23,470 & 246 & 506 & 407 & 205 & 11 \\
\hline 2025 & 34,450 & 20,380 & 307 & 381 & 315 & 383 & 16 \\
\hline 2030 & 45,890 & 14,250 & 238 & 394 & 118 & 37 & 3 \\
\hline$: 2035$ & $-53,100$ & 11,070 & 409 & 295 & 0 & 18 & 5 \\
\hline 2.040 & 66,350 & 6,640 & 15 & 163 & 14 & 82 & 1 \\
\hline
\end{tabular}


Table F. 27

CAPITAL CONSTRUCTION CASI FLOWS

IOR RISTRICTTD RICYCIS:--ALL REACTOR CASE

Large U Supply

(Millions of $\$ /$ Year)

\begin{tabular}{|c|c|c|c|c|c|c|c|}
\hline Year. & $\begin{array}{l}\text { lossil } \\
\text { Plants }\end{array}$ & $\begin{array}{l}\text { Nuclear } \\
\text { Plants } \\
\end{array}$ & $\begin{array}{c}\text { Fabrication } \\
\text { Plants } \\
\end{array}$ & $\begin{array}{c}\text { Reprocessing } \\
\text { Plants } \\
\end{array}$ & $\begin{array}{l}\text { Enrichnent } \\
\text { Plants } \\
\end{array}$ & $\begin{array}{r}U_{3}{ }^{0} 8 \\
\text { Mines } \\
\end{array}$ & $\begin{array}{l}\mathrm{ThO}_{2} \\
\text { Mines } \\
\end{array}$ \\
\hline 1970 & 5,383 & 34,510 & 27 & - & 133 & 213 & - \\
\hline 1975 & 8,870 & $51 ; 270$ & 29 & - & 189. & 292 & - \\
\hline 1980 & $7,819^{\circ}$ & 90,390 & 66 &.- & 321 & 502 & - \\
\hline 1985 & 3,564 & 14,680 & 131 & - & 545 & 810 & 1 . \\
\hline 1990 & 3,272 & $16 ; 850$ & 144 & 674 & 480 & 447 & 6 \\
\hline $1995^{\circ}$ & 4,663 & 23,180 & 204 & 597 & 507 & 794 & 11 \\
\hline 2000 & 4,970 & 26,970 & 267 & 635. & 574 & 766 & 9 \\
\hline 2005 & $5 ; 675$ & 32,910 & 500 & 546 & 481 & 641 & 19 \\
\hline 2010 & $6 ; 847$ & 39,400 & 726 & 733 & 821 & 567 & 25 \\
\hline 2015 & 8,054 & 39,390 & 621 & 838 & 524 & 721 & 21 \\
\hline 2020 & $11 ; 400$ & 41,560 & 838 & 1,031 & 372 & 266 & 28 \\
\hline 2025 & 16,790 & 43,550 . & 922 & 89.2 & 208 & 285 & 29. \\
\hline 2030 & 22,060 & 44,350 & 611 & 760 & 152 & 106 & 19 \\
\hline 2035 & 29,610 & 39,270 & 529 & 706 & 38 & 70 & 19 \\
\hline 2040 & 45,840 & 29,730 & 531 & 564 & 28 & 59 & 16 \\
\hline
\end{tabular}


Table F. 28

CAPITAL CONSTRUCTION CASH FLOWS

FOR IULL RLECYCLE--ALL REACTOR CASE

Large U Supply

(Millions of \$/Year)

\begin{tabular}{|c|c|c|c|c|c|c|c|}
\hline Year & $\begin{array}{l}\text { Fossil } \\
\text { plants }\end{array}$ & $\begin{array}{l}\text { Nuclear } \\
\text { Plants } \\
\end{array}$ & $\begin{array}{c}\text { Fabrication } \\
\text { Plants } \\
\end{array}$ & $\begin{array}{c}\text { Reprocessing } \\
\text { Plants } \\
\end{array}$ & $\begin{array}{l}\text { Enrichnent } \\
\text { Plants } \\
\end{array}$ & $\begin{array}{r}U_{3}{ }^{0} 8 \\
\text { Mines } \\
\end{array}$ & $\begin{array}{c}\mathrm{MnO}_{2} \\
\text { Mines }\end{array}$ \\
\hline 1970 & 5,380 & 3,450 & 27 & $\therefore$ & 133 & 213 & - \\
\hline 197.5 & 8,870 & $. .5,130$ & 29 & - & 189 & 292 & - \\
\hline 1980 & 7,820 & 9,040 & 66 & - & 321 & 502 & - \\
\hline 1985 & 3,560 & 14,670 & 131 & - & 548 & 810 & $: \quad-$ \\
\hline 1990 & 3,270 & 16,760 & 126 & 677 & 517 & 525 & 1 \\
\hline 1995 & 5,130 & $22 ; 470$ & 189 & .565 & 478 & 752 & 2 \\
\hline 2000 & 4,970 & 26,950 & 329 & 640 & 532 & .567 & 7 \\
\hline 2005 & 5,680 & 33,260 & 4.71 & 550 & 526 & 540 & 16 \\
\hline 2010 & 6,850 & 39,420 & 622 & 696 & 612. & 625 & 23 \\
\hline 2015 & 7,240 & 41,070 & 643 & 730 & 791 & 802 & 31 \\
\hline 2020 & 11,090 & 42,680 & 713 & 912 & 649 & 448 & 33 \\
\hline 2025 & 13,420 & 46,660 & 7.65 & 851 & 505 & 637 & 35 \\
\hline$\therefore 2030$ & 21,020 & 44,140 & 740 & 927 & 316 & 242 & $\therefore 34$ \\
\hline 2035 & 31,170 & 36,430 & 691 & 965 & 29 & 48 & $\therefore 30$ \\
\hline 2040 & 46,930 & 28,820 & 760 & 782 & 22 & 71 & 26 \\
\hline
\end{tabular}


Finally, yearly capital construction cash flows were calculated. The cash flows required for constructing fossil plants, nuclear plants, fabrication plants, reprocessing plants, enrichment plants, $\mathrm{U}_{3} \mathrm{O}_{8}$ mines and $\mathrm{ThO}_{2}$ mines are presented in Tables F.11 through F.14 for small uranium supply and Tables F.25 through F.28 for large uranium supply.

\section{Nuclean Plant Levelized Power Costs}

The components of the levelized power cost for a plant starting up in 2007 is given in Table F.29. In addition to the standard components.capital, fixed charges, $O \& M$, income tax, and fuel--the fuel cycle components are further subdivided into $U-233$, fissile plutonium, $U_{3} 0_{8}$, enrichment, fabrication, and reprocessing. These components were obtained from a single ALPS calculation in which all reactors were allowed to compete. The total levelized costs vary from $17.2 \mathrm{mills} / \mathrm{kwhr}$ for an FBR with a plutonium/thorium core and a thorium blanket to $24.0 \mathrm{mills} / \mathrm{kwhr}$ for a U-233 denatured CANDU. Although the CANDU's have a $3.6 \mathrm{mills} / \mathrm{kwhr}$ disadvantage in capital charge plus income tax with respect to the FWK, their fuel cycle was often significantly lower. Most reactors which rely entirely on uranium ore as a source of fissile fuel show a very high $\mathrm{U}_{3} \mathrm{O}_{8}$ cost--over $9 \mathrm{mills} / \mathrm{kwhr}$ for most plants. The slightly-enriched CANDU had the lowest fuel cycle cost $(6.7 \mathrm{mills} / \mathrm{kwhr})$, followed by the high-enriched HTGR ( $7.2 \mathrm{mills} / \mathrm{kwhr})$ and the low-enriched spectrum-shift PIR (7.7 mills/kwhr). The naturally-enriched CANDU had a fuel cycle cost of $8.8 \mathrm{mills} / \mathrm{kwhr}$, while the standard low-enriched PVR had a fuel cycle cost of $9.9 \mathrm{mills} / \mathrm{kwhr}$.

The denatured reactors in general had higher fuel cycle costs than the comparable conventional design. This is in part due to a higher 
Table F. 29

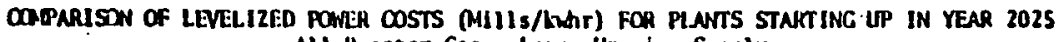
Al) Heactor Case--Large Uranilum Supply

Total Cost Coumonents

\begin{tabular}{|c|c|c|c|}
\hline Reactor & Total & Capital & $\begin{array}{l}\text { Fixed } \\
\text { giarges }\end{array}$ \\
\hline 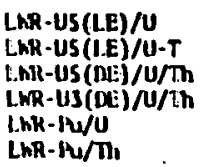 & $\begin{array}{l}19.3 \\
2.2 \\
20.7 \\
20.8 \\
19.2= \\
19.6\end{array}$ & $\begin{array}{l}6.4 \\
6.4 \\
6.4 \\
6.4 \\
6.4 \\
6.4\end{array}$ & $\begin{array}{l}.6 \\
.0 \\
.6 \\
.6\end{array}$ \\
\hline
\end{tabular}

SSOR-US(LE)/U SSTH-US(UE)/U/T

Imil-us(mat) N

ImR-US(SI:U) $/ N$

$\operatorname{limR-US(nE)/U/m}$

ImR-RuJu

InR-Pu/Th

inai-us (LE)/U-T

ITMR-US (IE) $)$

inca-us(ui:)/T

IIICK-U3 (UI:) $/ \mathrm{U} / \mathrm{T}$

ITCR-U3/7h

IIISG-PU/Th.

FER- $\boldsymbol{R}-10 / \mathrm{U}$

I:BR-AL-U/M,

18.27.

$19.3 \quad 7.1$

$22.8 \quad 9.3$

22.8
20.8
22.5
24.0

21.8
21.6

21.3
19.9

19.4

19.3

17.9

18.9

9.3
9.3
9.3

9.3

9.3

7.3

7.3

7.3

8.2
8.2

\section{queration}

Income
Tax

.9
.9
.9
.9
.9
.8

.9
.9
.9
.9
.9
.8

Tax Fuel

$\therefore$

1.1

.9

.

Funl Cost Cormonents

\begin{tabular}{|c|c|c|c|c|c|}
\hline$\underline{4233}$ & $\begin{array}{l}\text { Fissile } \\
\text { Pu } \\
\end{array}$ & $\mathrm{U}_{3} \mathrm{O}_{8}$ & Enriclument & Fabrication & Reprocessing \\
\hline $\begin{array}{r}0.0 \\
0.0 \\
-3.8 \\
9.0 \\
0.0 \\
-3.7\end{array}$ & $\begin{array}{r}-1.5 \\
0.0 \\
-0.8 \\
-0.9 \\
5.6 \\
12.0\end{array}$ & $\begin{array}{r}8.6 \\
9.6 \\
11.7 \\
0.0 \\
1.9 \\
0.0\end{array}$ & $\begin{array}{l}1.5 \\
1.6 \\
2.8 \\
0.0 \\
.0 \\
0.0\end{array}$ & $\begin{array}{l}.5 \\
.1 \\
.7 \\
2.6 \\
1.6 \\
1.5\end{array}$ & $\begin{array}{l}.8 \\
.3 \\
.8 \\
.8 \\
.8 \\
.7\end{array}$ \\
\hline $\begin{array}{r}0.0 \\
6.1 \\
-1.1\end{array}$ & $\begin{array}{l}-1.9 \\
-0.7 \\
11.4\end{array}$ & $\begin{array}{l}7.2 \\
0.0 \\
.0 .0\end{array}$ & $\begin{array}{l}1.2 \\
0.0 \\
0.0\end{array}$ & $\begin{array}{l}.5 \\
2.4 \\
1.5\end{array}$ & $\begin{array}{l}.8 \\
.7 \\
.7\end{array}$ \\
\hline $\begin{array}{r}0.0 \\
0.0 \\
-6.8 \\
5.4 \\
0.0 \\
-6.4\end{array}$ & $\begin{array}{r}-3.1 \\
-1.7 \\
-0.2 \\
-0.3 \\
0.5 \\
10.6\end{array}$ & $\begin{array}{r}7.8 \\
6.1 \\
10.7 \\
0.0 \\
3.9 \\
0.0\end{array}$ & $\begin{array}{r}.0 \\
.3 \\
2.6 \\
0.0 \\
.0 \\
0.0\end{array}$ & $\begin{array}{r}1.1 \\
.6 \\
.8 \\
3.1 \\
1.9 \\
1.9\end{array}$ & $\begin{array}{l}3.0 \\
1.4 \\
1.4 \\
1.4 \\
1.4 \\
1.4\end{array}$ \\
\hline $\begin{array}{r}0.0 \\
0.0 \\
-1.5 \\
-2.7 \\
6.6 \\
1.8 \\
-1.1\end{array}$ & $\begin{array}{r}0.0 \\
-0.5 \\
-.3 \\
-.0 \\
-0.3 \\
-.0 \\
0.3\end{array}$ & $\begin{array}{l}8.2 \\
7.1 \\
7.7 \\
6.8 \\
0.0 \\
0.0 \\
0.0\end{array}$ & $\begin{array}{l}1.7 \\
1.5 \\
1.9 \\
1.8 \\
0.0 \\
0.0 \\
0.0\end{array}$ & $\begin{array}{l}.6 \\
.7 \\
.6 \\
.5 \\
1.7 \\
1.4 \\
1.1\end{array}$ & $\begin{array}{r}.2 \\
.6 \\
.5 \\
.7 \\
0.9 \\
1.2 \\
.2\end{array}$ \\
\hline $\begin{array}{r}0.0 \\
-3.5\end{array}$ & $\begin{array}{l}3.1 \\
5.5\end{array}$ & $\begin{array}{l}0.0 \\
0.0\end{array}$ & $\begin{array}{l}0.0 \\
0.0\end{array}$ & $\begin{array}{l}2.4 \\
2.4\end{array}$ & $\begin{array}{l}1.6 \\
1.5\end{array}$ \\
\hline
\end{tabular}


enrichment component of the fuel cycle cost. The denatured U-235 fueled PIV's have the highest enrichment component--2.8 milis/kwhr.

The cost penalty for fabricating fuels containing U-23J is reflected in significantly higher fabrication cost components for reactors loading this fuel. On the other hand, the reprocessing cost component was in general about $1 \mathrm{mill} / \mathrm{kwhr}$ for most reactors. The exception to this was the CAVDJ, where the lower discharge exposure results in a higher reprocessing cost component.

The fuel cycle componet which varied the most from reactor to reactor was the fissile fuel component. The U-233 component varied from a cost of $12.6 \mathrm{mills} / \mathrm{kwhr}$ to a credit of $11.3 \mathrm{mills} / \mathrm{kwhr}$. The fissile plutonium component varied from 13.1 to $-5.0 \mathrm{mills} / \mathrm{kwhr}$. 
Appendix A

THORIUM ASSESSIENT PROGRAM SYSTEM STUDIES

(Material Flows and Nuclear Power Growth Patterns) 


\section{THIS PAGE \\ WAS INTENTIONALLY \\ LEFT BLANK}


MATERIAL FLOWS AND GROWTH PATTERNS

Material flows diagrams for all cases of the Thorium Assessment Program Systems Study are presented in the following figures to show the impact of various fuel cycle policy decisions and reactor strategy options on uranium ore requirements and enrichment requirements. Fissile isotopes, thorium and heavy metal flows and relative market penetration by reactor type are also presented. The summation of heavy metal flows into reactors indicates fabrication plant requirement and out of reactors the reprocessing (or waste disposal for throwaway cycle) plant requirements. The year 2035 was chosen as a time when the system appeared to be in quasi-equilibrium--that is, U-233 and fissile plutoniun stockpiles are near zero. All the above-mentioned material flows have been normalized to a total system capacity of 1 Give.

The installed nuclear capacity table following each material flow diagram presents nuclear capacity information for four specific years-$2000,2030,2035$ and 2040--to show the trends of 1) total installed nuclear capacity (GWe), 2) the percent of that capacity in dispersed areas outside secure energy centers, and 3) the market penetration by each reactor type available in each particular case. 


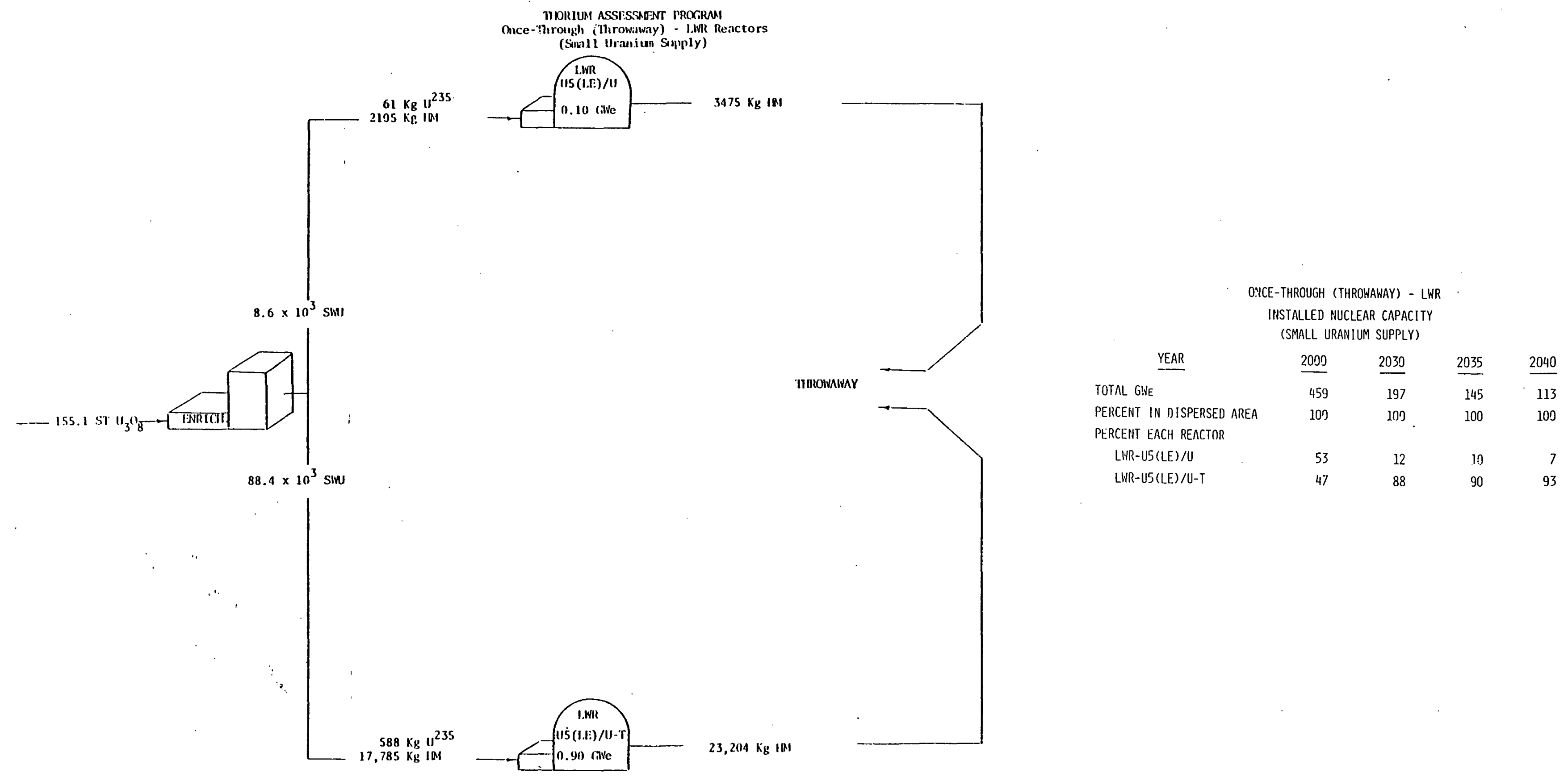




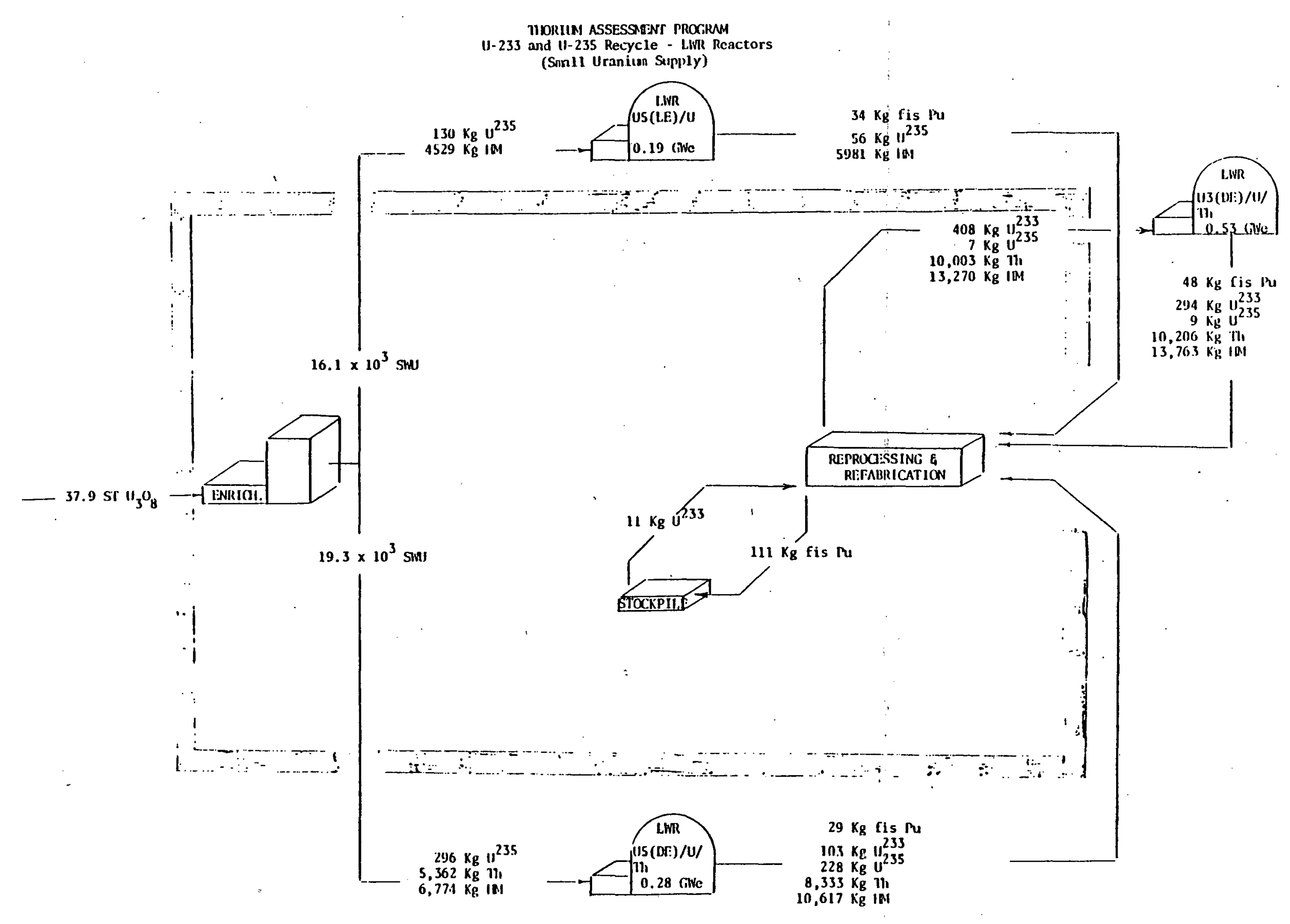

\begin{tabular}{|c|c|c|c|c|}
\hline \multicolumn{5}{|c|}{$\begin{array}{l}\text { U-233 AND U-235 RECYCLE - LHR } \\
\text { INSTALLED NUCLEAR CAPACITY } \\
\text { (SMALL URANIUM SUPPLY) }\end{array}$} \\
\hline YEAR & 2000 & 2030 & 2035 & 2040 \\
\hline TOTAL GWE & 490 & 369 & 280 & 202 \\
\hline PERCENT IN dISPERSED aREA & 100 & 109 & 100 & 100 \\
\hline PERCENT EACH REACTOR & & & & \\
\hline LHR-U5(LE)/U & 79 & 20 & 19 & 29 \\
\hline LWR-UL(DE)/U/TH & 20 & 36 & 28 & 23 \\
\hline LWR-U3(DE)/U/TH & 1 & 44 & 53 & 57 \\
\hline
\end{tabular}




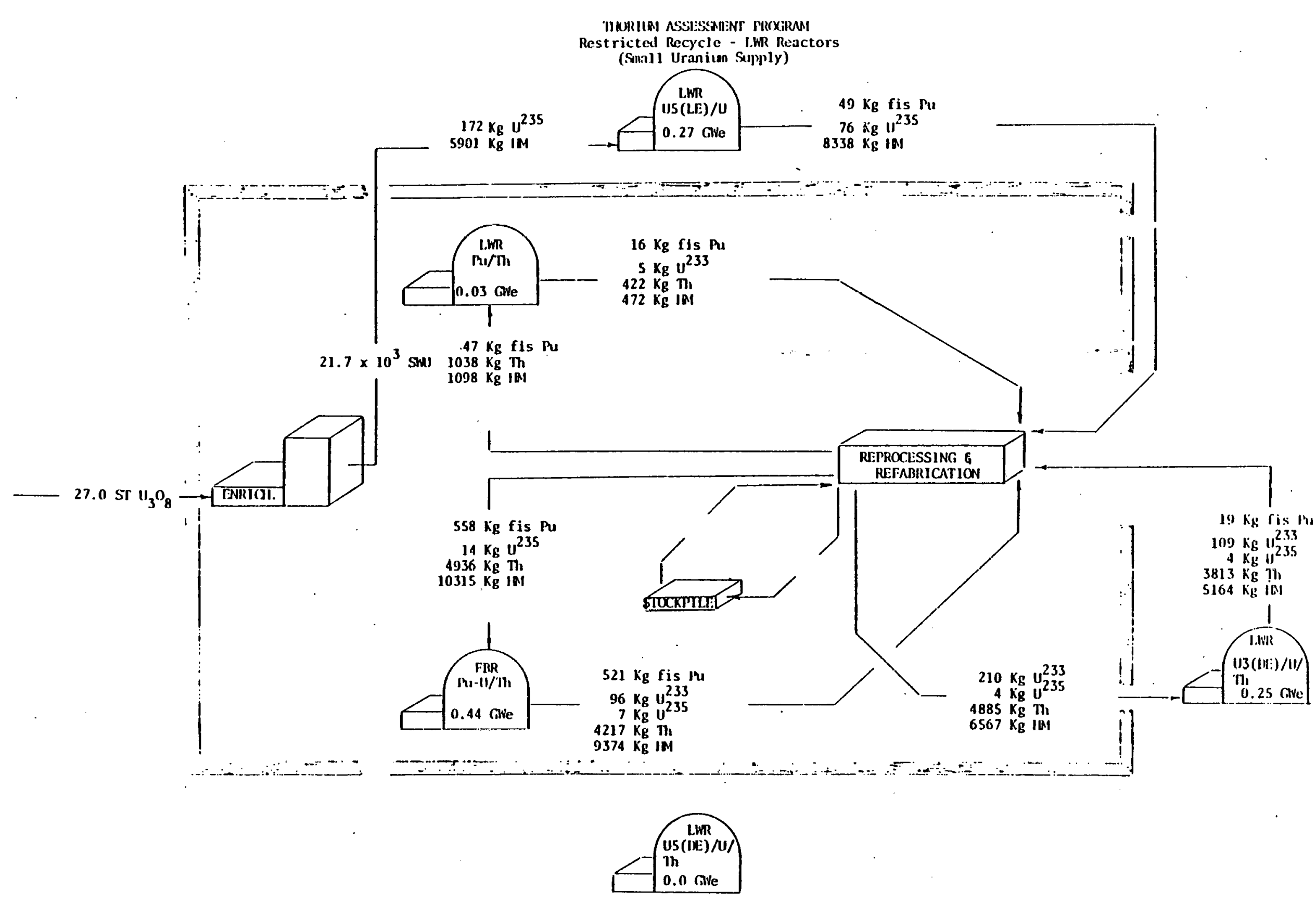

\begin{tabular}{|c|c|c|c|c|}
\hline \multicolumn{5}{|c|}{$\begin{array}{l}\text { RESTRICTED RECYCLE - LUR } \\
\text { INSTALLED. IUUCLEAR CAPACITY } \\
\text { (SMALL URNIIU'I SUPPLY) }\end{array}$} \\
\hline YEAR & 2000 & 2030 & 2035 & 2040 \\
\hline TOTAL GWE & 584 & 1249 & 1255 & 1243 \\
\hline $\begin{array}{l}\text { PERCENT IN DISPERSED AREA } \\
\text { PERCENT EACH REACTOR }\end{array}$ & 95 & 59 & 52 & 46 \\
\hline. LWR-US(LE)/U & 95 & 39 & 27 & 16 \\
\hline LIRR-UZ(DE)/U/TH & - & 20 & 25 & 30 \\
\hline I.WR-Pu/TH & 5 & 3 & 3 & 7 \\
\hline FBR-PU $-U / / T_{H}$ & - & 38 & 44 & 46 \\
\hline
\end{tabular}




\begin{tabular}{|c|c|c|c|c|}
\hline \multicolumn{5}{|c|}{$\begin{array}{l}\text { FULL MECYYLLE - LHR } \\
\text { INSTALLED NUCLEAR CAPACITY } \\
\text { (SMALL URANIUM SUPPLY) }\end{array}$} \\
\hline YEAR & 2000 & 2030 & 2035 & 2040 \\
\hline TOTAL GWE & 589 & 1201 & 1280 & 1295 \\
\hline PERCENT IN DISPERSED AREA & 94 & 34 & 24 & 14 \\
\hline \multicolumn{5}{|l|}{ PERCENT EACH REACTOR } \\
\hline LWR-U5(LE)/U & 94 & 34 & 24 & 14 \\
\hline LWR-Pu/U & 6 & 23 & 33 & 43 \\
\hline FBR-PU-U/U & - & 42 & 43 & 42 \\
\hline
\end{tabular}




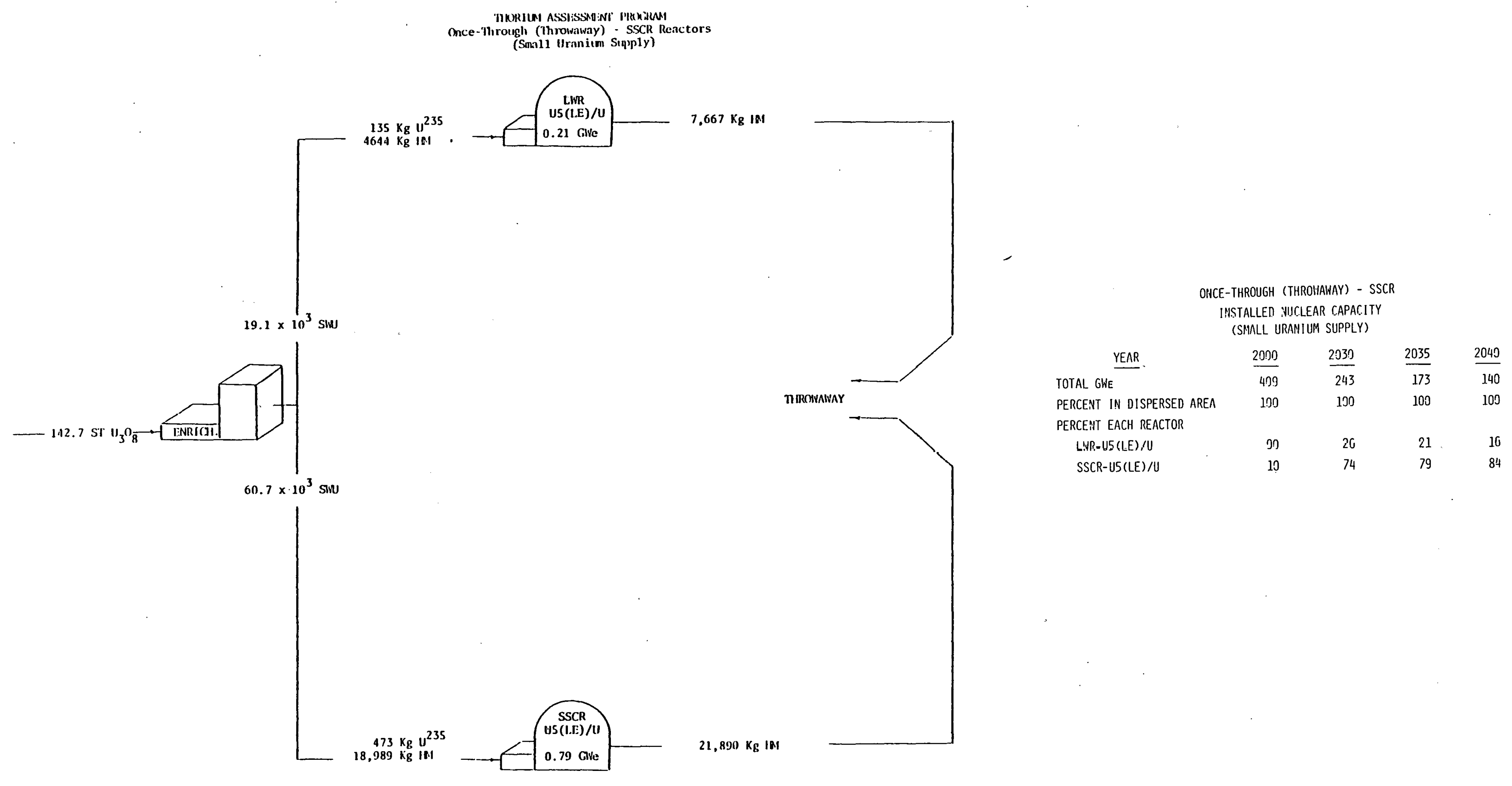




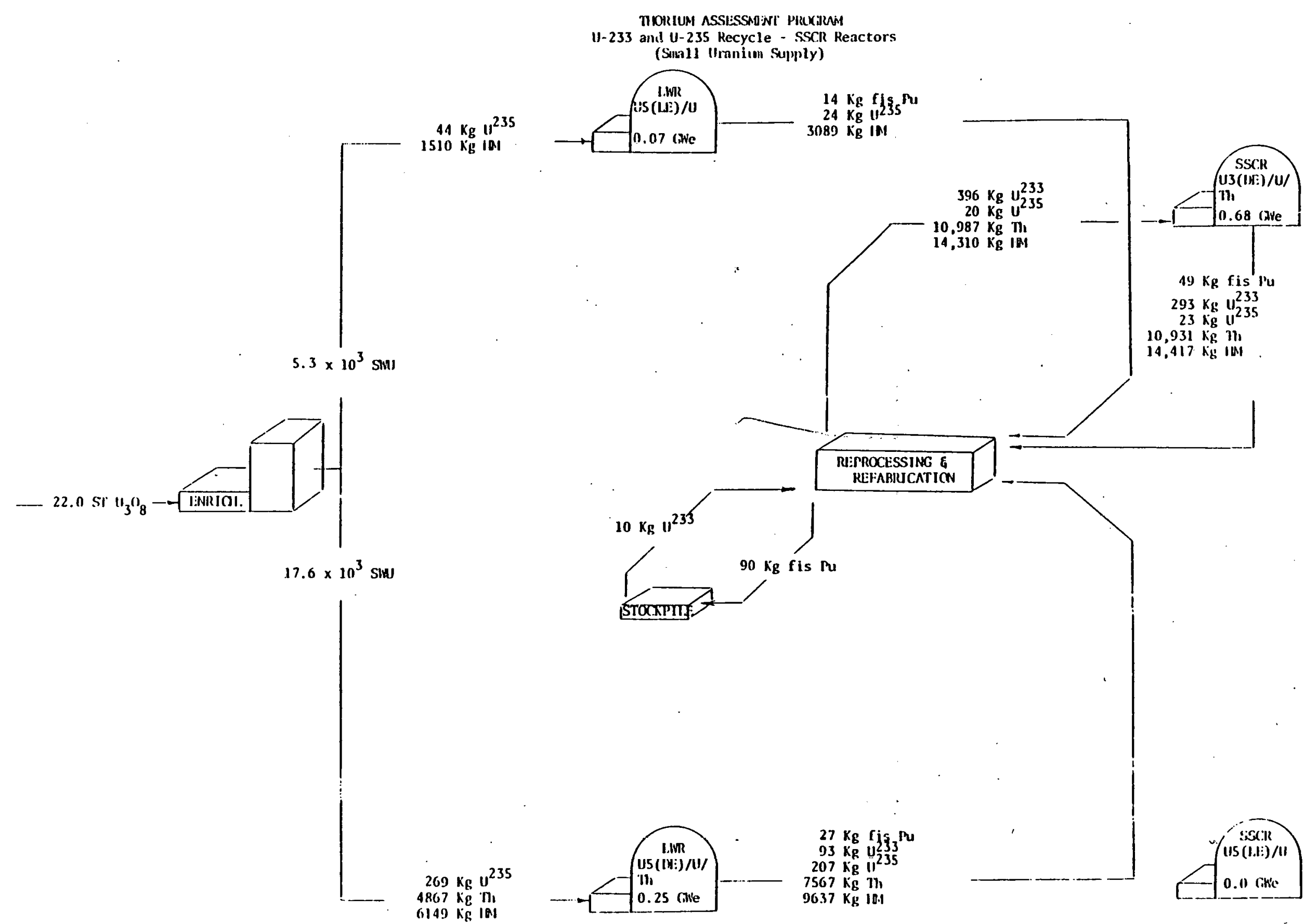

\begin{tabular}{|c|c|c|c|c|}
\hline \multirow[b]{2}{*}{ YEAR } & \multicolumn{3}{|c|}{$\begin{array}{l}\text { U-233 NAID U-235 RECYCLE - SSCR } \\
\text { INSTALLED NUCLEAR CAPACITY } \\
\text { (SMALL URANIUM SUPPLY) }\end{array}$} & $\cdots$ \\
\hline & 2000 & 2030 & 2035 & 2040 \\
\hline TOTAL GWE & 471 & 575 & 457 & 347 \\
\hline $\begin{array}{l}\text { PERCENT IN DISPERSED AREA } \\
\text { PFRCFNI ACH PEACTR }\end{array}$ & 100 & 103 & 100 & 100 \\
\hline $\begin{array}{l}\text { PERCENT EACH REACTOR } \\
\text { LHR-U5(LE)/U }\end{array}$ & 73 & 9 & 7 & \\
\hline LHR-U5(DE)/U/TH & 25 & 34 & 25 & 20 \\
\hline $\operatorname{SSCR}-U 3(\mathrm{DE}) / \mathrm{U} / \mathrm{TH}_{\mathrm{H}}$ & 2 & 57 & 68 & 75 \\
\hline
\end{tabular}




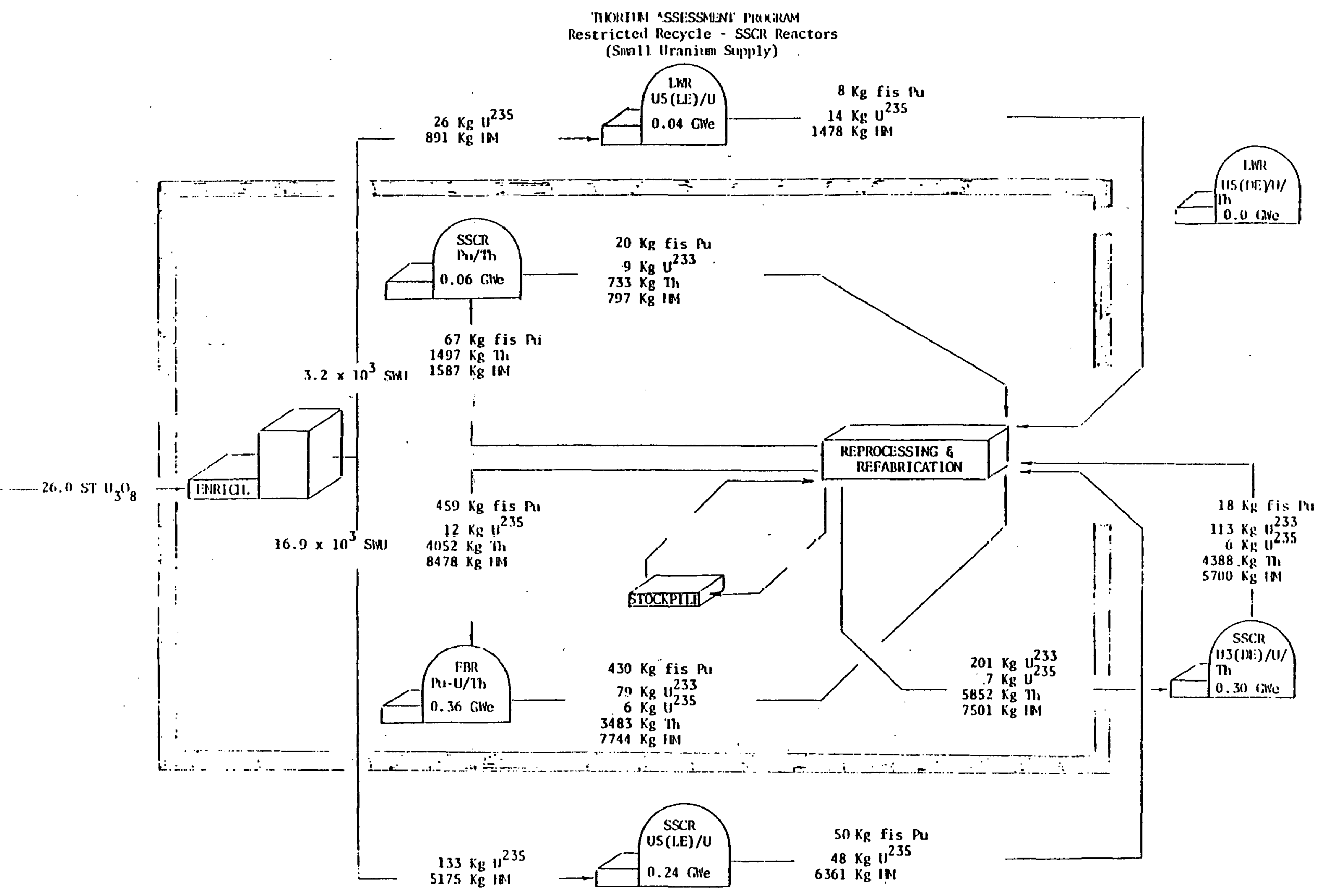

\begin{tabular}{|c|c|c|c|c|}
\hline \multicolumn{5}{|c|}{$\begin{array}{l}\text { RESTRICTED RECYCLE - SSCR } \\
\text { I.ISTALLED !UUCLEAR CAPACITY } \\
\text { (SMMLLL URANIUM SUPPLY) }\end{array}$} \\
\hline YEAR & 2099 & 2030 & 2035 & 2040 \\
\hline TOTAL GHE & 558 & 1536 & 1605 & 1626 \\
\hline PERCENT IN DISPERSED AREA & 94 & 64 & 58 & 54 \\
\hline PERCENT EACH REACTOR & & & & \\
\hline LWR-US (LE)/U & 85 & 7 & 4 & 2 \\
\hline LWR-U5(DE)/U/IH & 1 & - & - & - \\
\hline SSCR-U5(LE)/U & 7 & 31 & 24 & 15 \\
\hline$S S C R-U 3(D E) / U / T_{H}$ & 1 & 25 & 30 & 37 \\
\hline SSCR-PU/TH & 6 & 3 & 6 & 9 \\
\hline FER-PU-U/TH & - & 33 & 36 & 37 \\
\hline
\end{tabular}




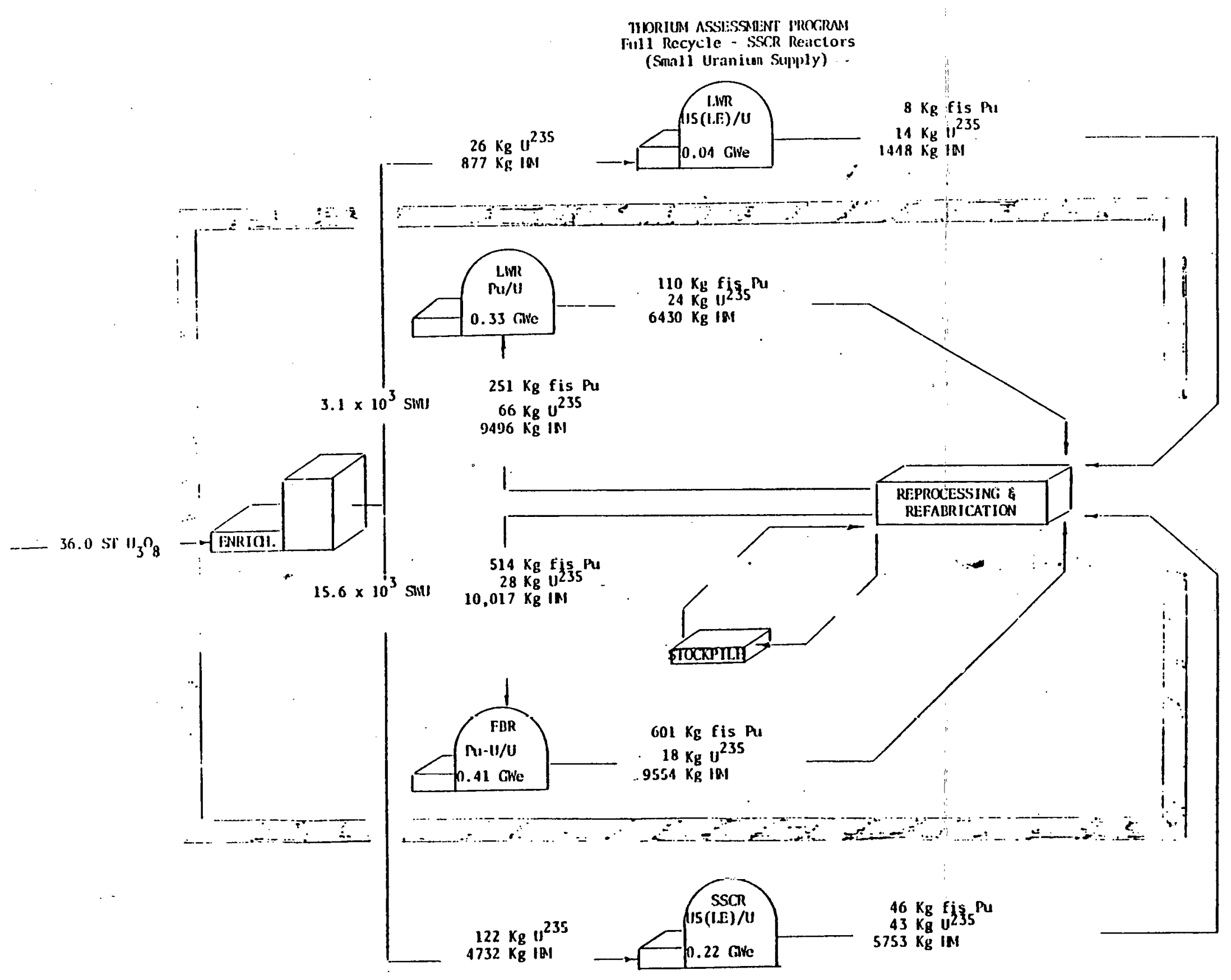

\begin{tabular}{|c|c|c|c|c|}
\hline \multicolumn{5}{|c|}{$\begin{array}{l}\text { FULL RECYCLE - SSCR } \\
\text { INSTALLED NUCLEAR CAPACITY } \\
\text { (SMALL URANIUM SUPPLY) }\end{array}$} \\
\hline YEAR & 2000 & 2030 & 2035 & 2040 \\
\hline TOTAL GWE & 543 & 1453 & 1552 & 1556 \\
\hline PERCENT IN DISPERSED AREA & 93 & 37 & 26 & 16 \\
\hline PERCENT EACH REACTOR & & & & \\
\hline LWR-U5(LE)/U & 86 & 7 & 4 & 2 \\
\hline L:WRR-Pu/U & 7 & 22 & 33 & 43 \\
\hline SSCR-US(LE)/U & 7 & 30 & 22 & 13 \\
\hline FBR-Pu-U/U & - & $40^{-}$ & 41 & 41 \\
\hline
\end{tabular}


78
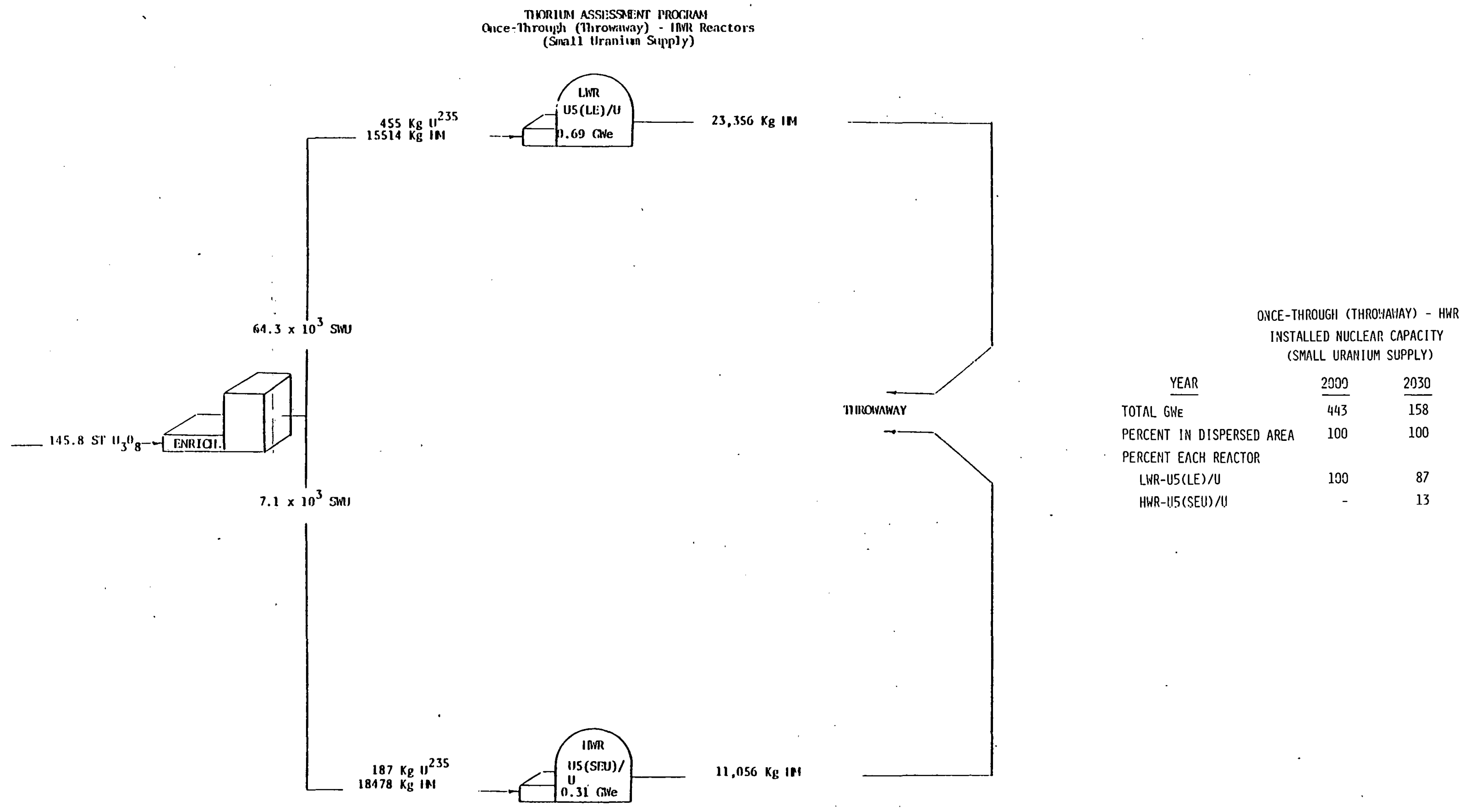

$\begin{array}{cc}\frac{2035}{129} & \frac{2040}{121} \\ 100 & .100 \\ 69 & 49 \\ 31 & 51\end{array}$




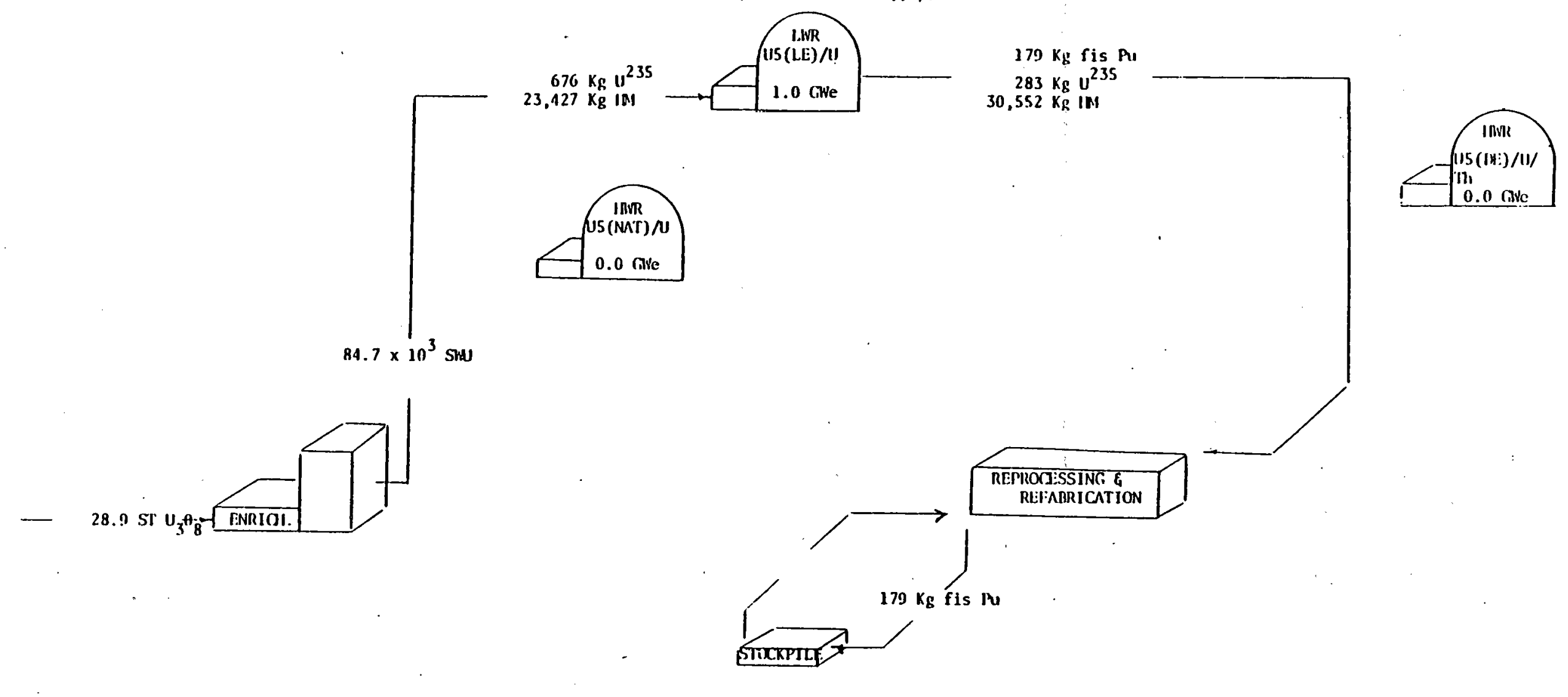

\begin{tabular}{|c|c|c|c|c|}
\hline \multirow[t]{2}{*}{ U- } & \multicolumn{2}{|c|}{$\begin{array}{l}\text { U-233 AiND U-235 RECYCLE - HW } \\
\text { INSTALLED IIUCLEAR CAPACIYY } \\
\text { (SMALL URANIUM SUPPLY) }\end{array}$} & \multirow[b]{2}{*}{2035} & \multirow[b]{2}{*}{204} \\
\hline & 2000 & 2030 & & \\
\hline TOTAL GNE & 484 & 273 & 199 & $\overline{150}$ \\
\hline $\begin{array}{l}\text { PERCENT IN DISPERSED AREA } \\
\text { PERCENT EACH REACTOR. }\end{array}$ & 100 & 100 & 100 & 100 \\
\hline LWR-U5(LE)/U & 100 & 100 & 100 & 100 \\
\hline
\end{tabular}

다료



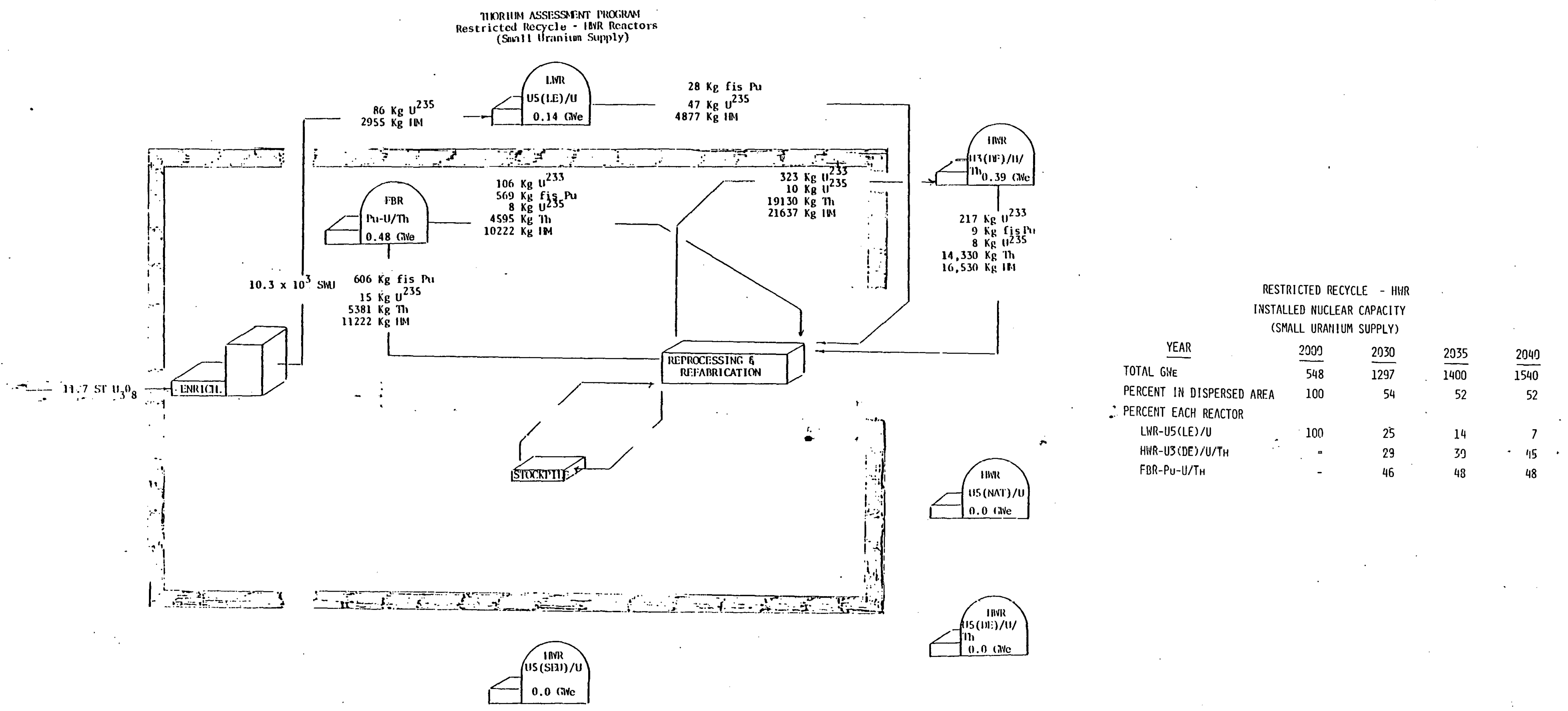


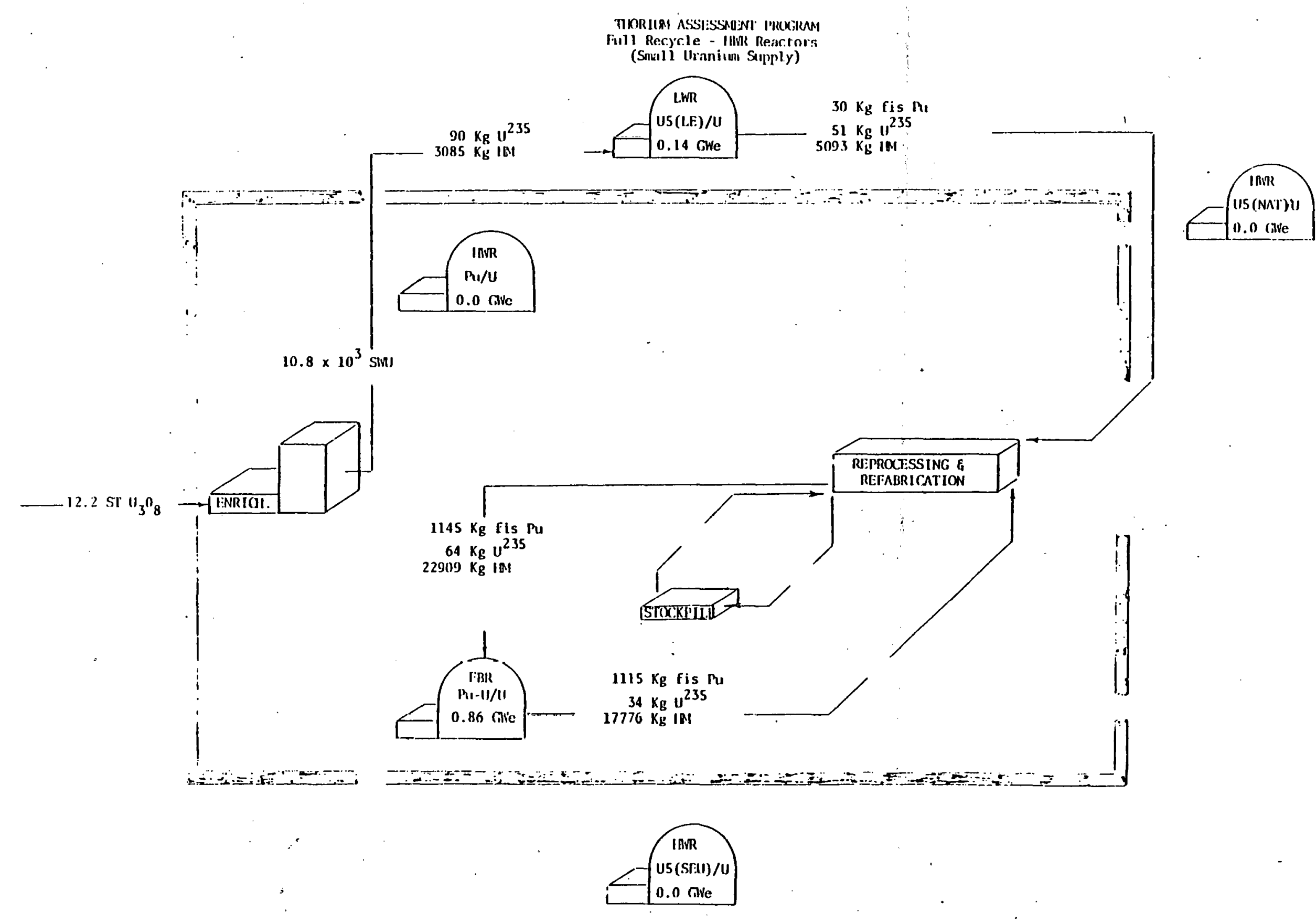

FULL RECYCLE - HH:R IHSTALLED :UUCLEAR CAPACITY

\begin{tabular}{|c|c|c|c|c|}
\hline YEAR & 2000 & 2030 & 2035 & 2040 \\
\hline TOTAL GWE & 548 & 1230 & 1367 & 1603 \\
\hline PERCENT IN DISPERSED AREA & 100 & 27 & 14 & 7 \\
\hline PERCENT EACH REACTOR & & & & \\
\hline LWR-U5(LE)/U & 100 & 27 & 14 & \\
\hline FBR-Pu-U/U & “- & 73 & 86 & \\
\hline
\end{tabular}


82
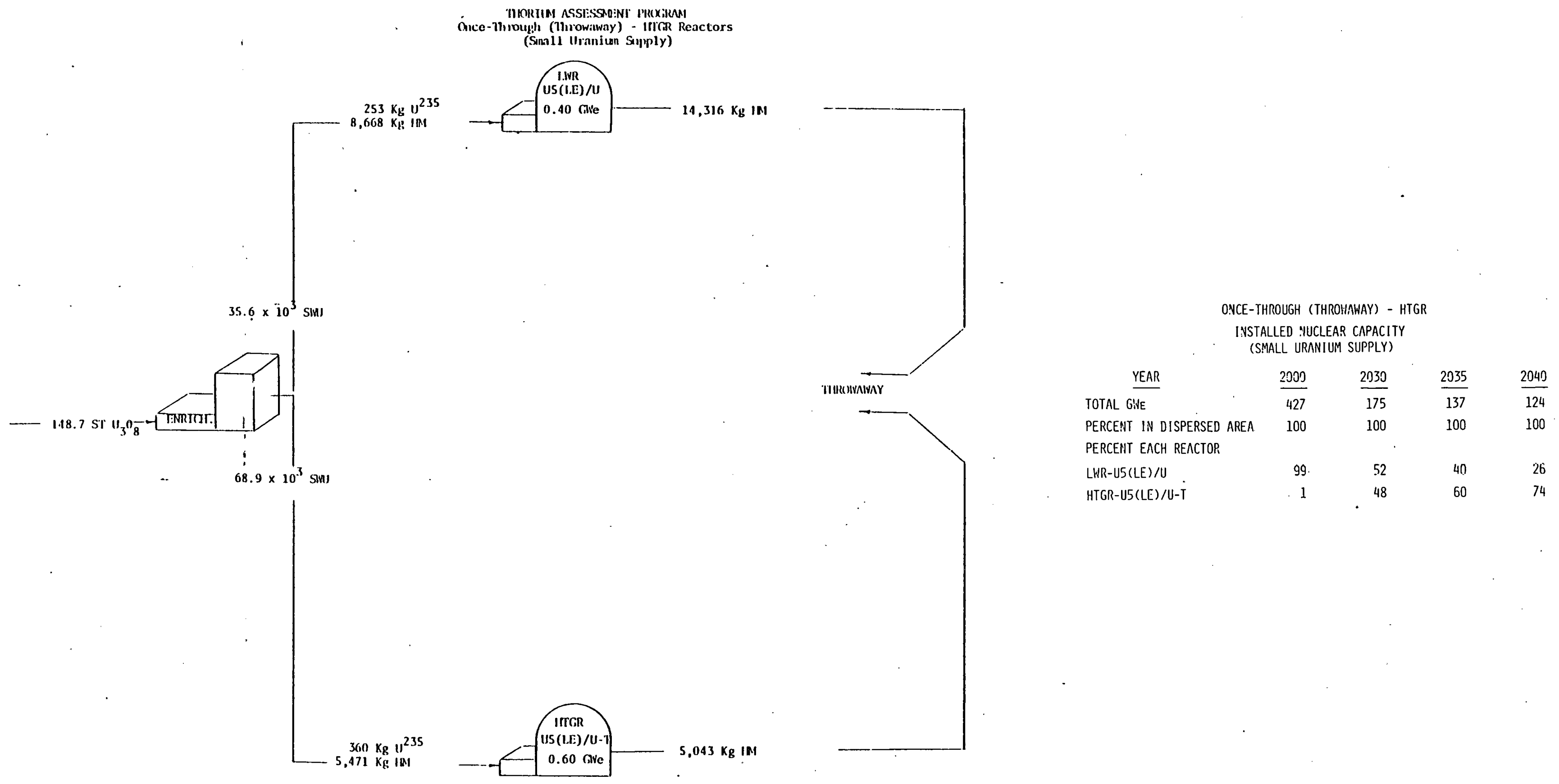


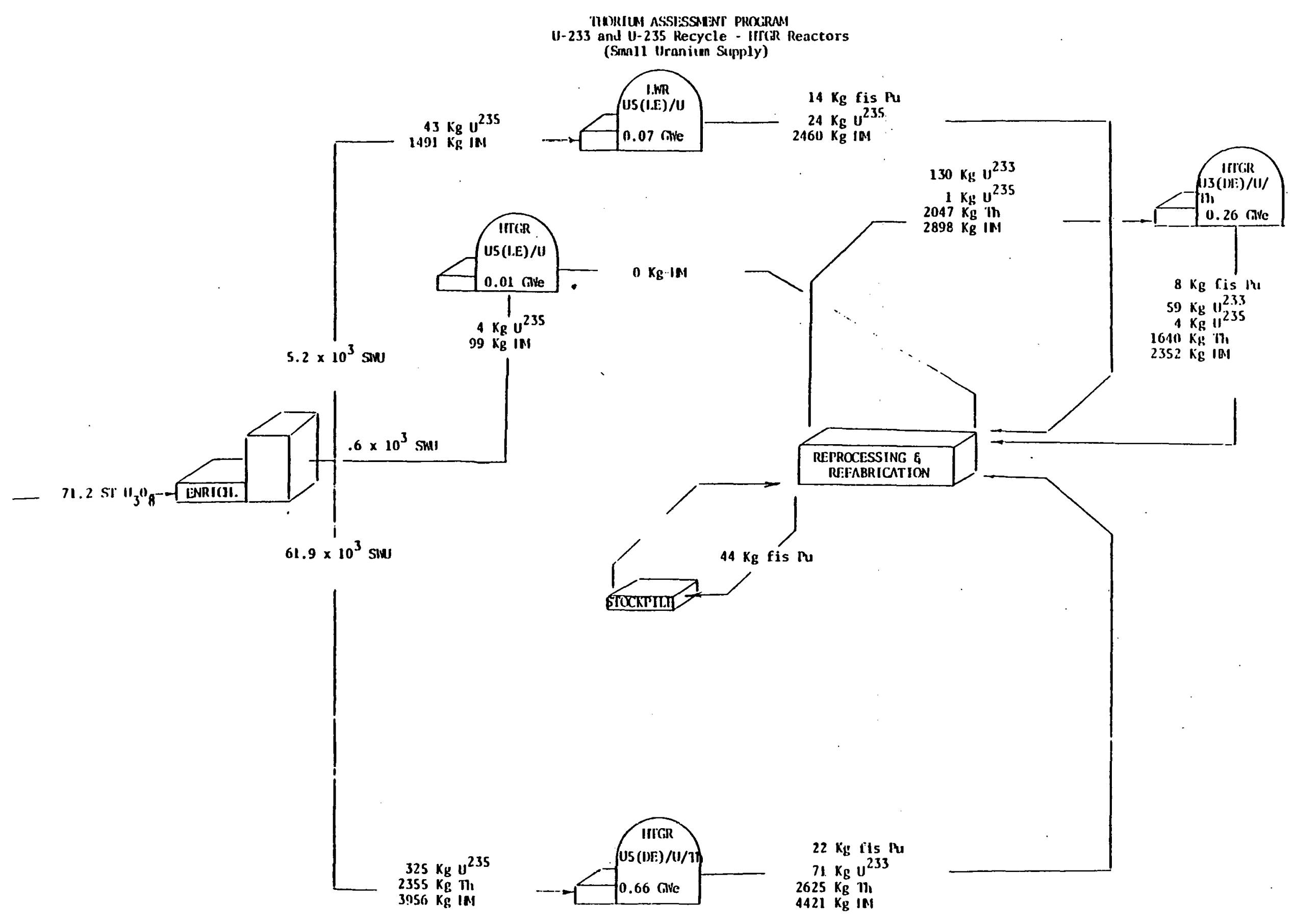

\begin{tabular}{|c|c|c|c|c|}
\hline \multicolumn{5}{|c|}{$\begin{array}{l}\text { U-233 A:ID U-235 RECYCLE - HTGR } \\
\text { INSTALLED NUCLEAR CAPACITY } \\
\text { (SMALL URANIUM SUPPLY) }\end{array}$} \\
\hline YEAR & 2000 & 2030 & 2035 & 2040 \\
\hline TOTAL GNE & 417 & 749 & 708 & $\overline{560}$ \\
\hline PERCENT IN DISPERSED AREA & 100 & 100 & 100 & 100 \\
\hline PERCENT EACH REACTOR & & & & \\
\hline LWR-US (LE)/U & 98 & 11 & 7 & 5 \\
\hline HTGR-US(LE)/U & . & - & 1 & 3 \\
\hline HTGR-U5(DE)/U/TH & 2 & 68 & 66 & 57. \\
\hline HTGR-U3(DE)/U/TH & & 21 & 26 & 36 \\
\hline
\end{tabular}




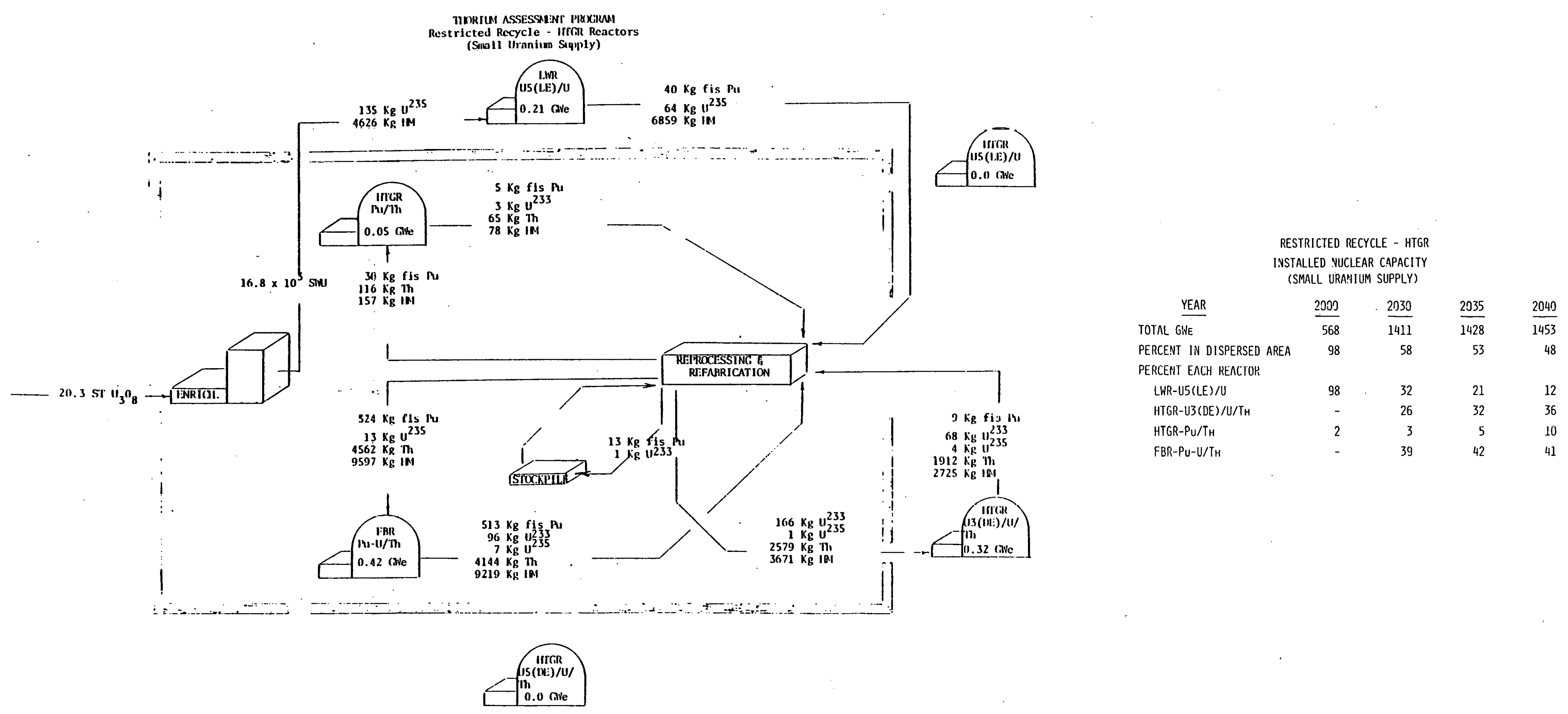




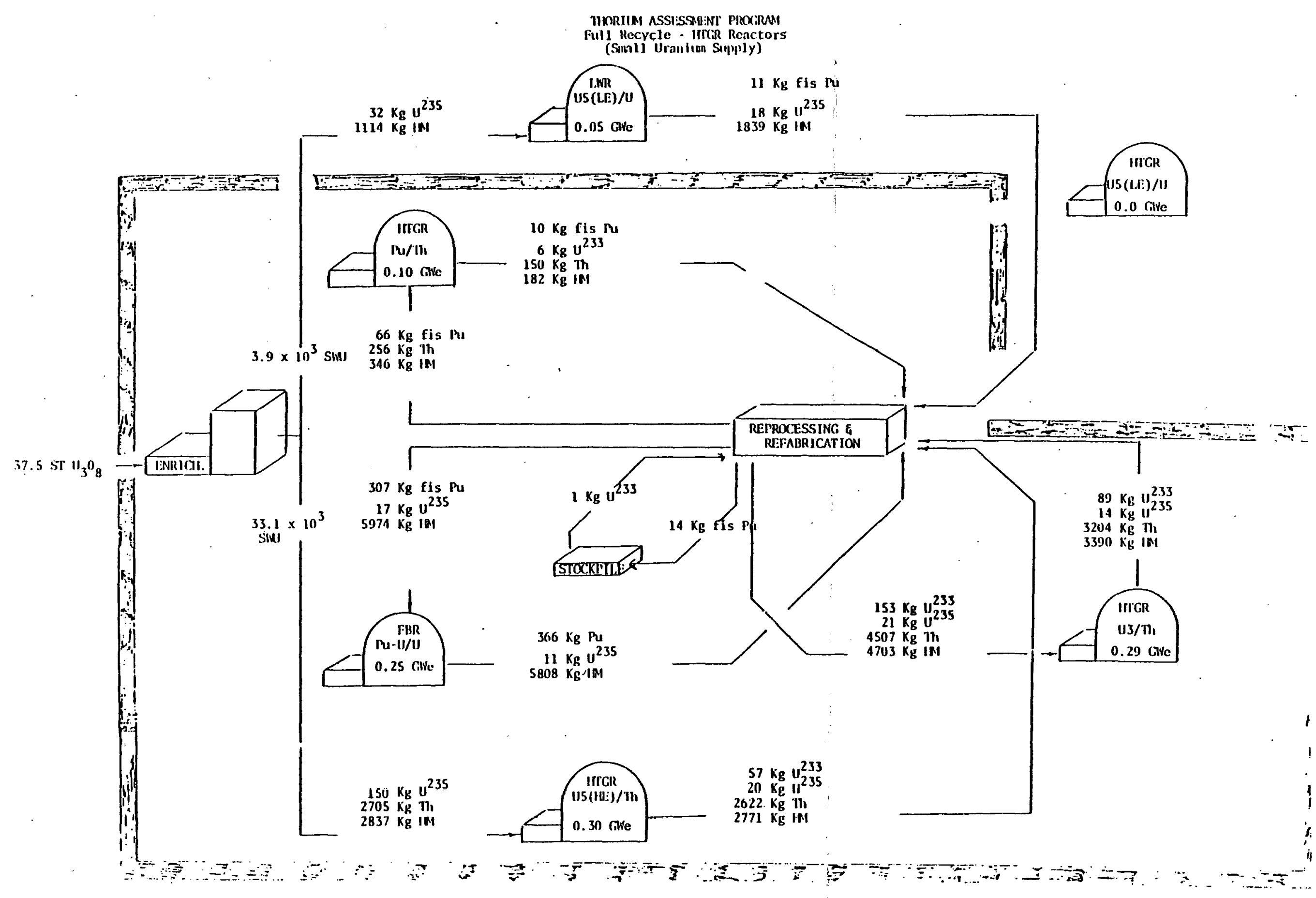

\begin{tabular}{|c|c|c|c|c|}
\hline \multicolumn{5}{|c|}{$\begin{array}{l}\text { FULL RECYCLE - HTGR } \\
\text { I:STALLED IUCLEAR CAPACITY } \\
\text { (SMALL URANIUM SUPPLY) }\end{array}$} \\
\hline YEAR & 2000 & 2030 & 2035 & 2040 \\
\hline TOTAL GWE & 539 & 1592 & 1744 & 1807 \\
\hline PERCENT IN DISPERSED AREA & 97 & 9 & 5 & \\
\hline PERCENT EACH REACTOR & & & & \\
\hline LWR-U5(LE)/U & 97 & 9 & 5 & \\
\hline HTGR-US(HE)/TH & 2 & 33 & 30 & 25 \\
\hline HTGR-U3/TH & $=$ & 24 & 29 & 34 \\
\hline HTGR-PU/TH & 2 & 7 & 10 & 15 \\
\hline FBR-Pu-U/U & - & 26 & 25 & 24 \\
\hline
\end{tabular}




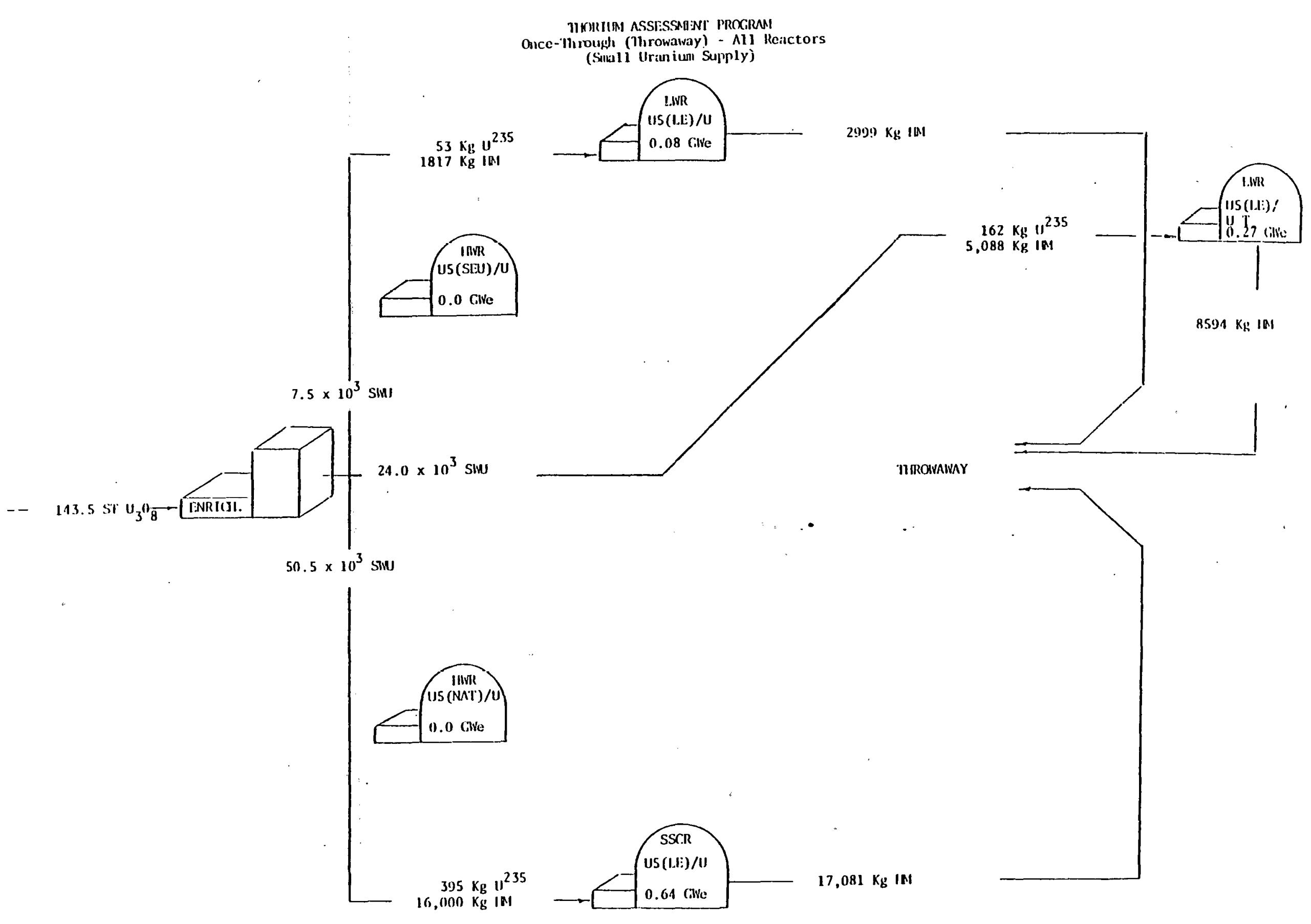




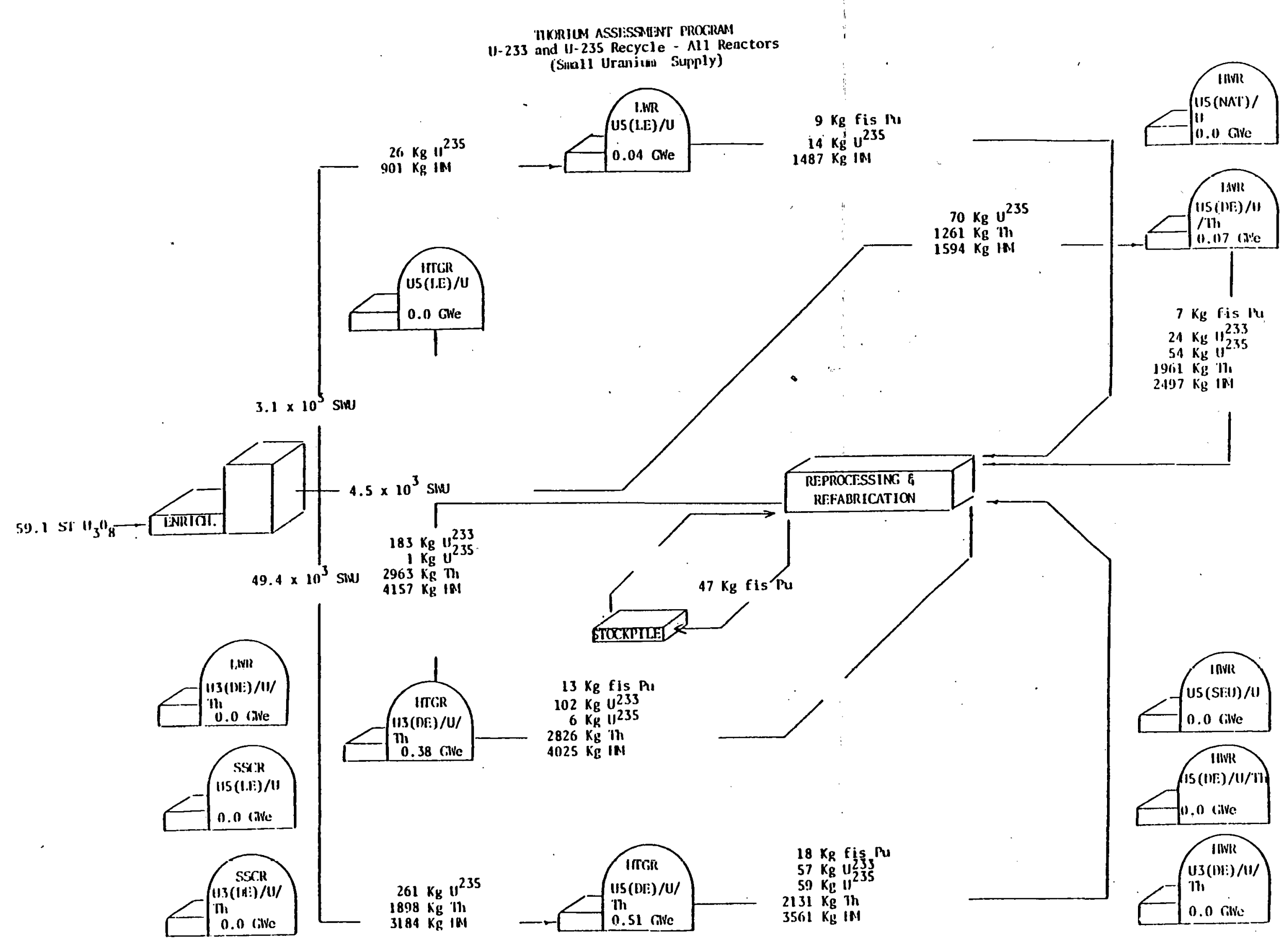

\begin{tabular}{|c|c|c|c|c|}
\hline $\begin{array}{l}3 \text { A } \\
\text { INS }\end{array}$ & \multicolumn{3}{|c|}{$\begin{array}{l}\text { WD U-235 RECYCLE - ALL REA } \\
\text { ISTALLED NUCLEAR CAPACITY } \\
\text { (SMALL URANIUM SUPPLY) }\end{array}$} & \multirow[b]{2}{*}{2040} \\
\hline YEAR & 2000 & 2030 & 2035 & \\
\hline TOTAL GHE & 426 & 777 & 688 & 542 \\
\hline PERCENT liv DISPERSED AREA & 100 & 100 & 100 & 100 \\
\hline \multicolumn{5}{|l|}{ PERCENT EACH REACTOR } \\
\hline LHR-U5(LE)/U & 78 & 6 & 4 & 3 \\
\hline LIIR-US(DE)/U/TH & 13 & 10 & 7 & 5 \\
\hline HTGR-U5(LE)/U & - & - & - & 2 \\
\hline $\mathrm{H}[G R-U 5(\mathrm{DE}) / \mathrm{U} / \mathrm{HH}$ & 2 & 50 & 51 & 48 \\
\hline HTGR-U3(DE)/U/TH & 2 & 34 & 38 & 43 \\
\hline
\end{tabular}




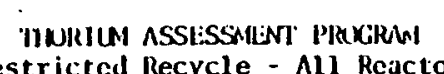

(Small Uraniun Simply)
(I)

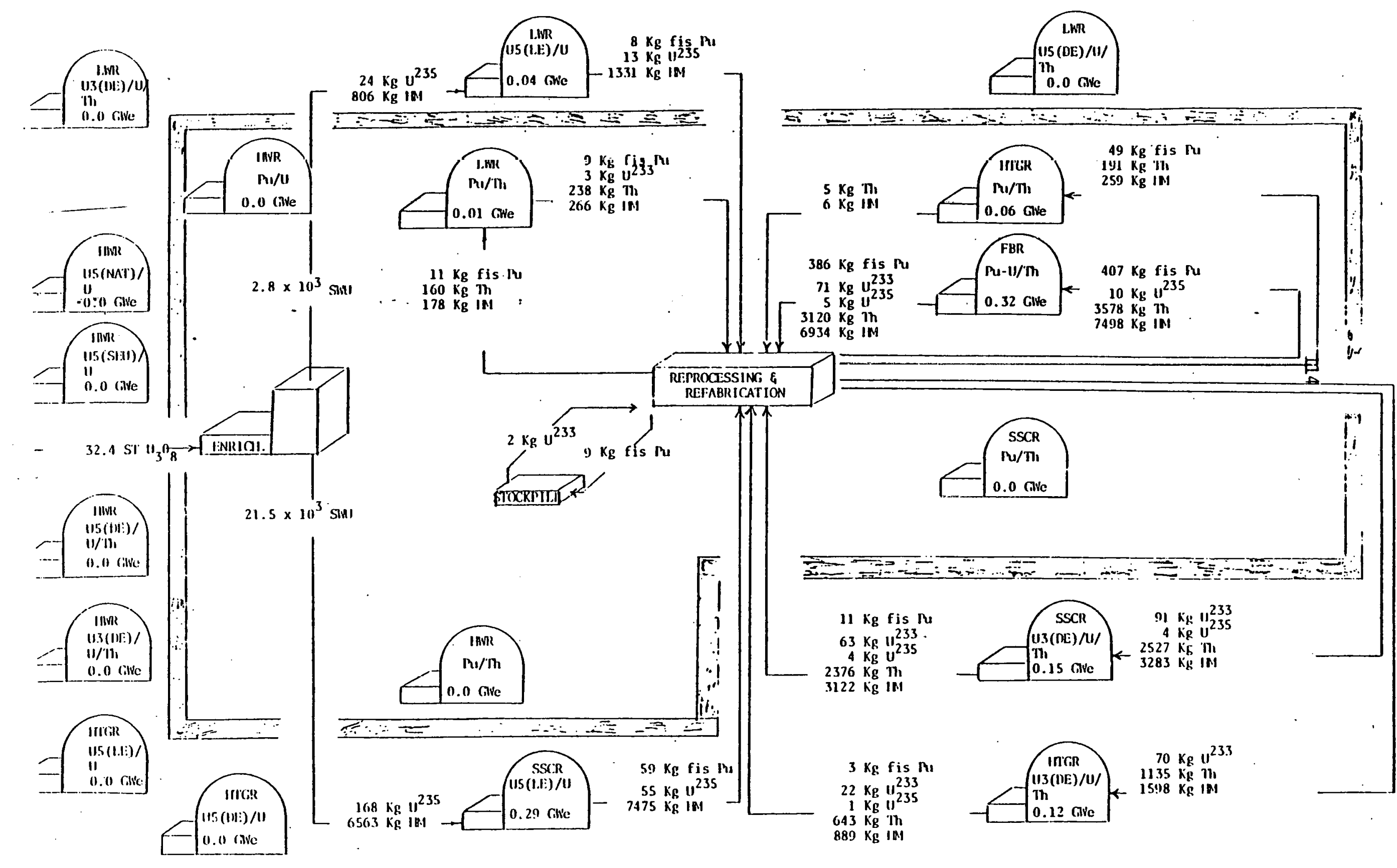

\begin{tabular}{|c|c|c|c|c|}
\hline \multirow[b]{2}{*}{ YEAR } & \multicolumn{3}{|c|}{$\begin{array}{l}\text { FULL RECYCLE - ALL REACTORS } \\
\text { IIISTALLED NUCLEAR CAPACITY } \\
\text { (SMALL URANIUM SUPPLY) }\end{array}$} & \\
\hline & 2000 & 2030 & 2035 & 2040 \\
\hline TOTAL GME & 555 & 1627 & 1734 & 1743 \\
\hline PERCENT IN DISPERSED AREA & 90 & 13 & 7 & 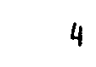 \\
\hline \multicolumn{5}{|l|}{ PERCENT EACH REACTOR } \\
\hline LWR-U5(LE)/U & 83 & 6 & 3 & \\
\hline Li:R-Pu/U & 6 & 6 & 3 & \\
\hline SSCR-U5(LE)/U & 7 & 7 & 4 & \\
\hline HTGR-US(HE)/TH & 2 & 30 & 27 & 2 \\
\hline HTGR-U3/TH & 0 & 21 & 26 & 3 \\
\hline IITGR-PU/TH & 2 & 5 & 11 & 1 \\
\hline FBr-Py-U/U & 0 & 25 & 25 & \\
\hline HUR-U3(NAT)/U & - & - & - & \\
\hline HWR-U5 (SEU)/U & - & - & - & \\
\hline HIMR-PU/U & - & - & ـ & \\
\hline HIIR-U5(LE)/U & .- & - & ـ & \\
\hline
\end{tabular}




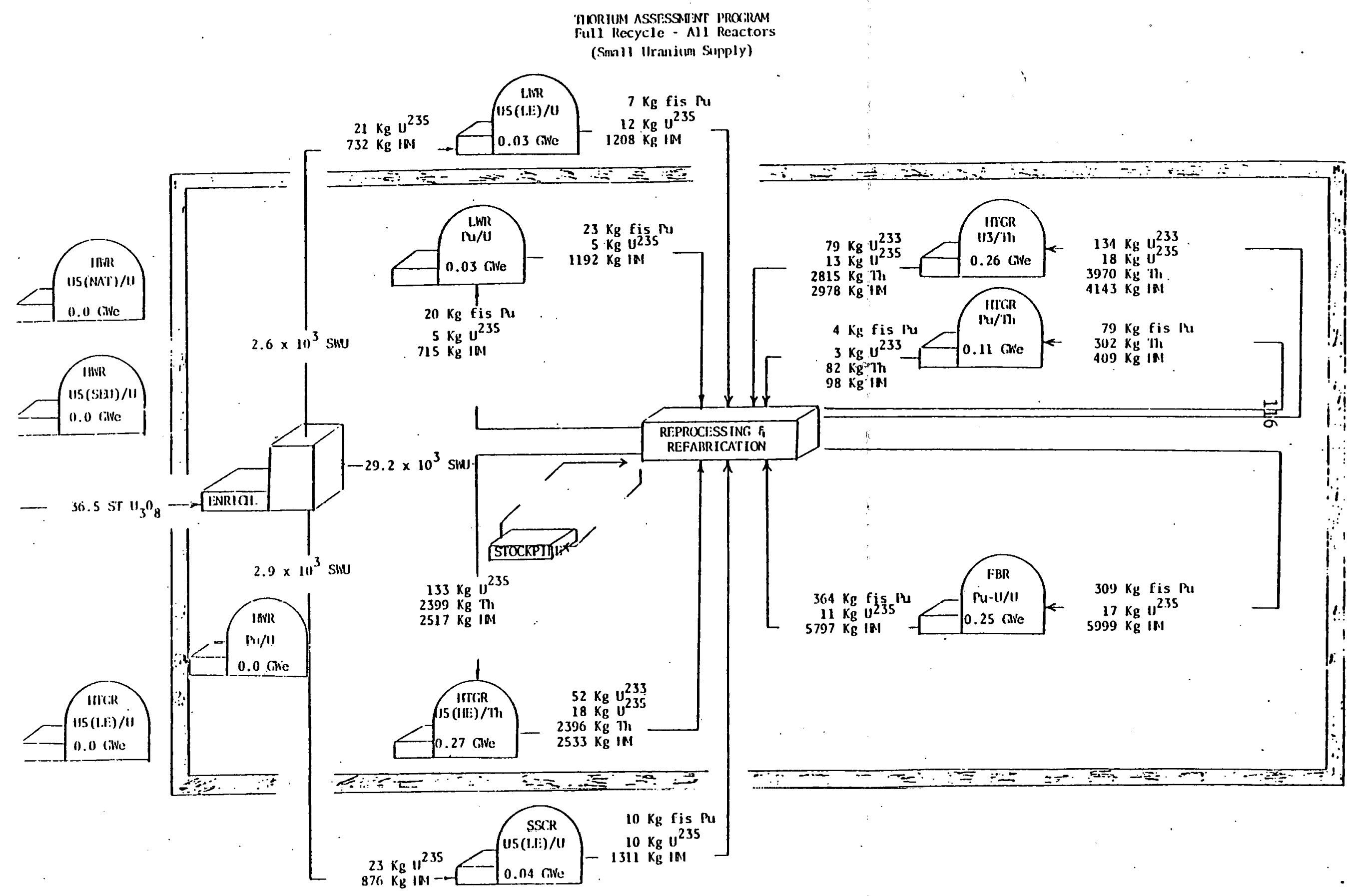




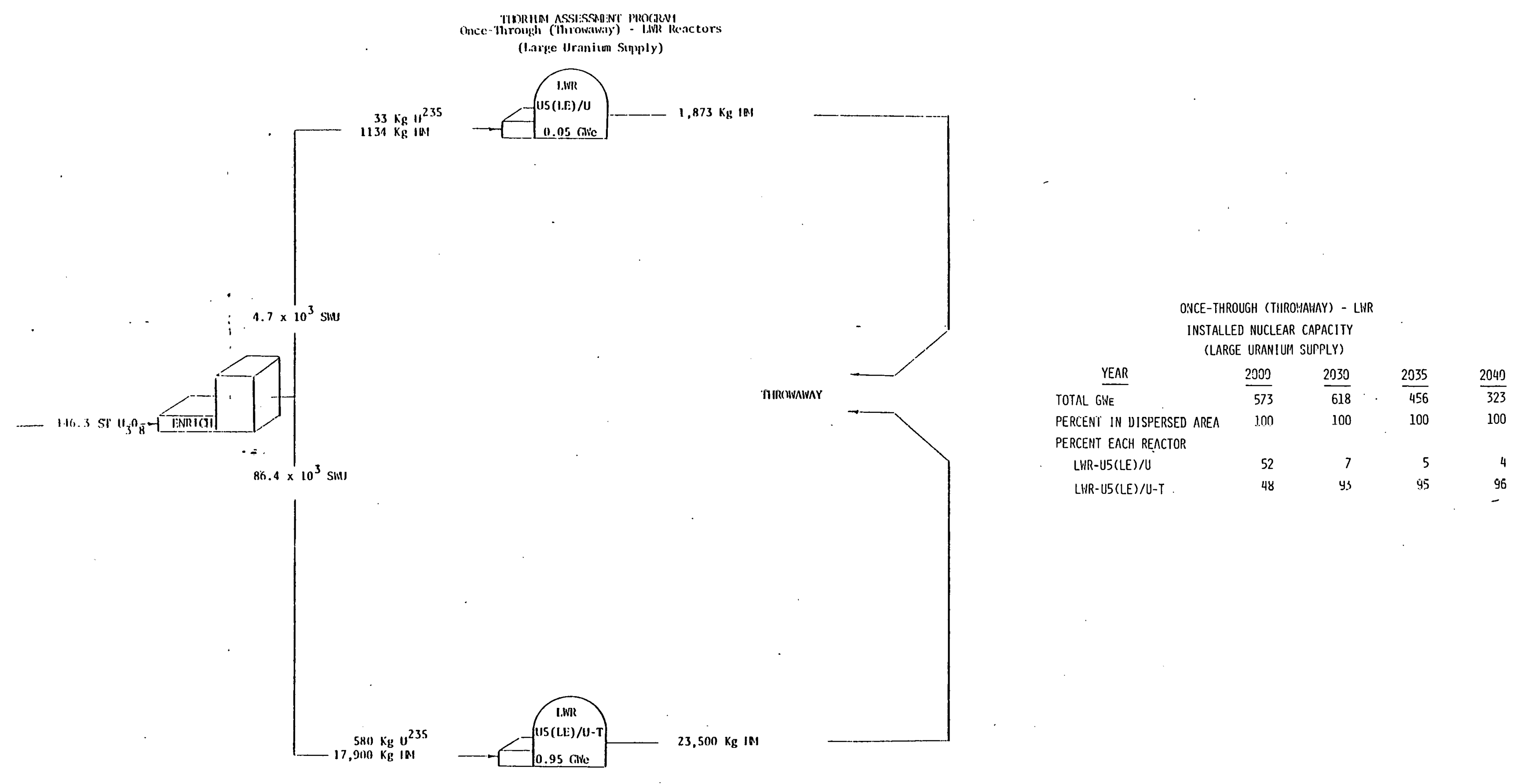




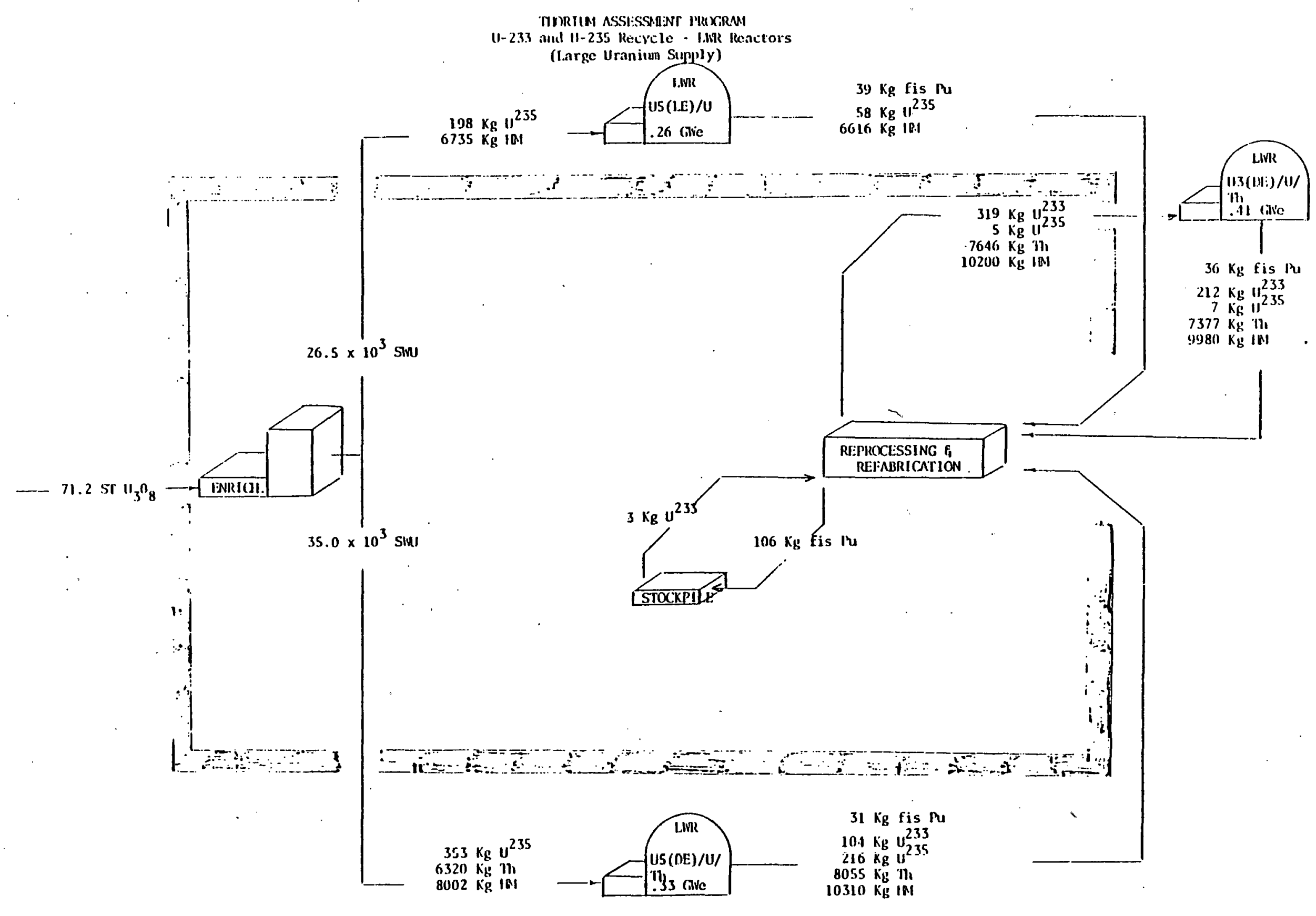

\begin{tabular}{lcccc}
\multicolumn{5}{c}{ U-233 AHD U-235 RECYCLE - LLIR } \\
IHSTALLED NUCLEAR CAPACITY \\
(LLRGE URANIUM SUPPLY)
\end{tabular}




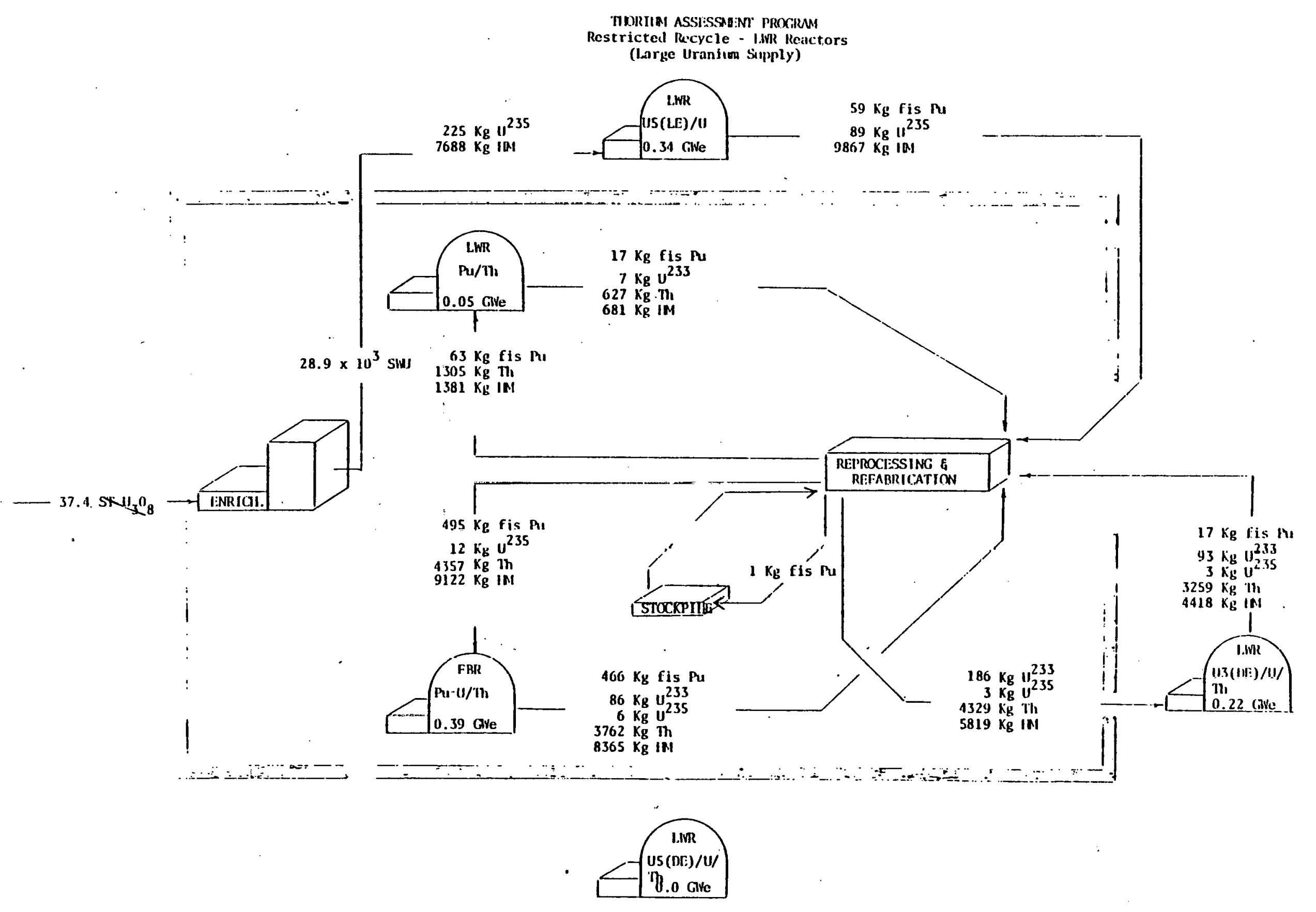

RESTRICTED RECYCLE - LHR

INSTALLED NUCLEAR CAPACITY

\begin{tabular}{|c|c|c|c|c|}
\hline YEAR & 2000 & 2030 & 2035 & 2040 \\
\hline TOTAL GiNE & 634 & 1683 & 1708 & 1675 \\
\hline $\begin{array}{l}\text { PERCENT IN DISPERSED AREA } \\
\text { PERCENT EACH REACTOR }\end{array}$ & 95 & 62 & 56 & 5 \\
\hline LWR-U5(LE)/U & 95 & 46 & 34 & \\
\hline $\mid W R-\|Z(n F) /\| / T H$ & - & 17 & 22 & 2 \\
\hline LLR-Pu/TH & 5 & 3 & 5 & \\
\hline FBR-Pu-J//TH & & 35 & 39 & \\
\hline
\end{tabular}




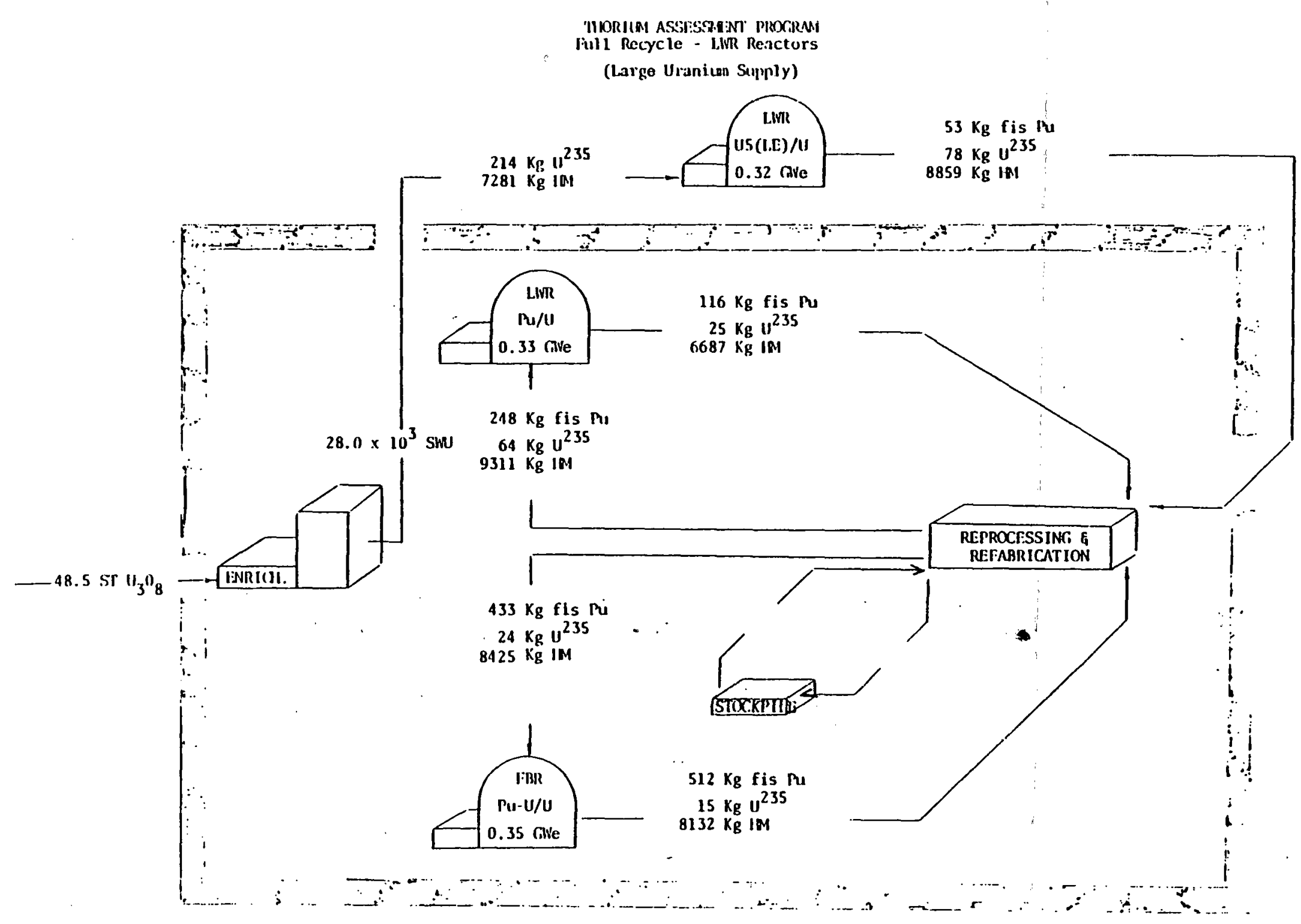

\begin{tabular}{|c|c|c|c|c|}
\hline \multicolumn{5}{|c|}{$\begin{array}{l}\text { FULL RECYCLE - LHR } \\
\text { INSTALLED HUCLEAR CAPACITY } \\
\text { (LARGE URANIUM SUPPLY) }\end{array}$} \\
\hline YEAR & 2000 & 2030 & 2035 & 2040 \\
\hline TOTAL GWE & 634 & 1709 & $\overline{1798}$ & 1775 \\
\hline PERCENT IN DISPERSED AREA & 94 & 42 & 32 & 23 \\
\hline PERCENT EACH REACTOR & & & & \\
\hline LWR-U5(LE)/U & 94 & 42 & 32 & 23 \\
\hline LWR-Pu/U & 6 & 24 & 33 & 42 \\
\hline FBR-Pu-U/U s & - & 34 & 35 & 35 \\
\hline
\end{tabular}




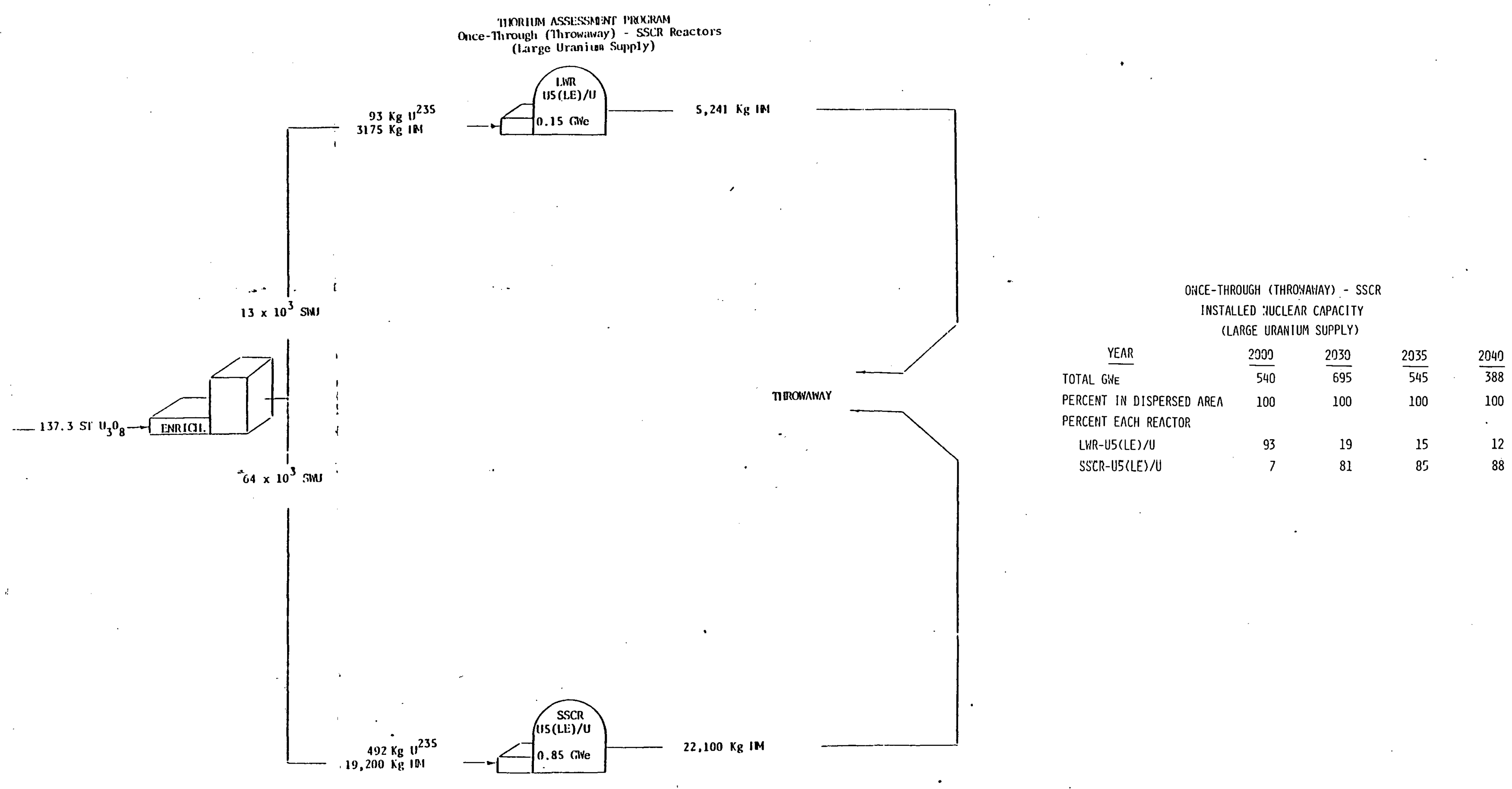




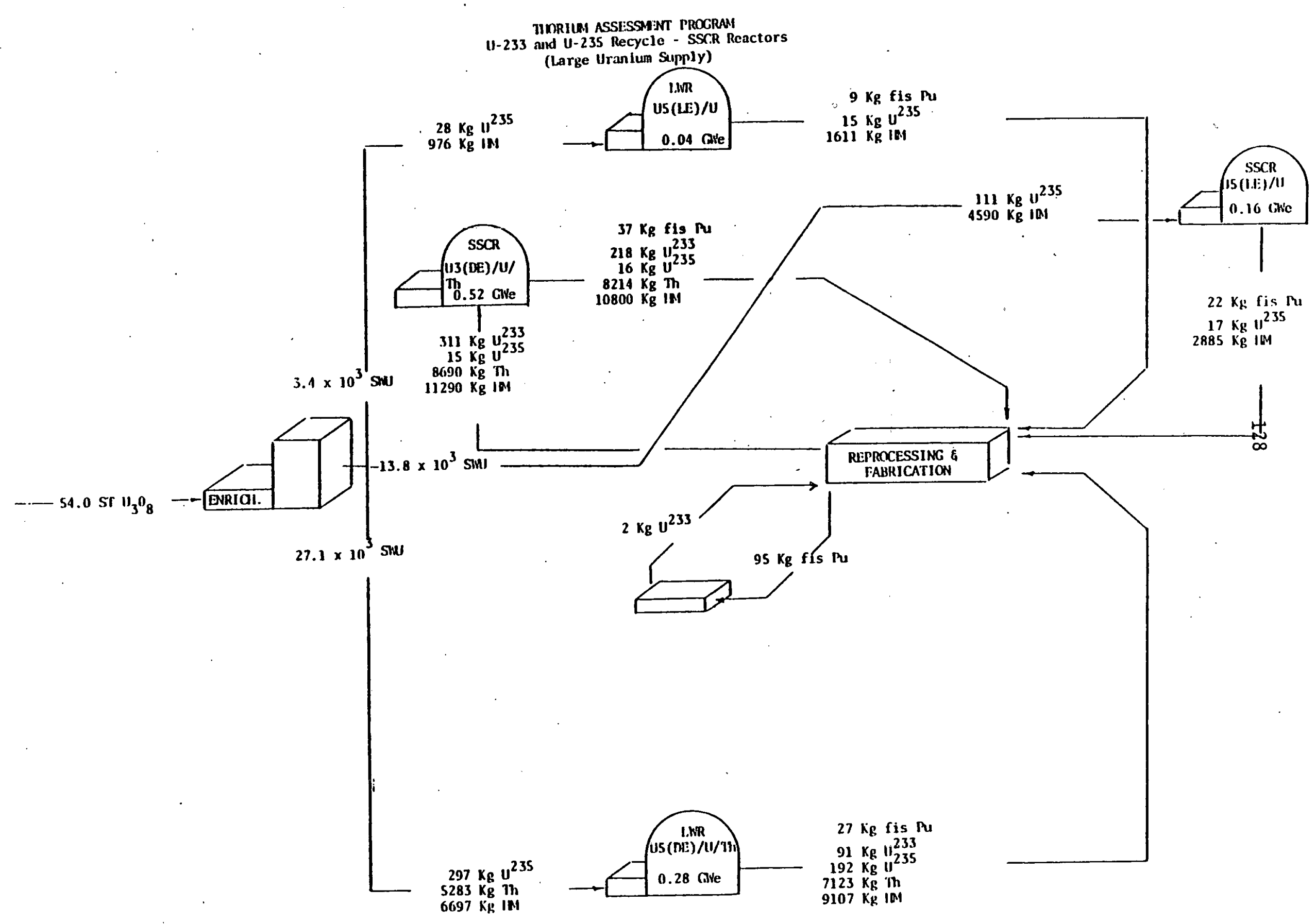

\begin{tabular}{|c|c|c|c|c|}
\hline \multicolumn{5}{|c|}{$\begin{array}{l}\text { U-233 AND U-235 RECYCLE - SSCR } \\
\text { I:ISTALLD :UCLEAR CAPACITY } \\
\text { (LARGE URANIUM SUPPLY) }\end{array}$} \\
\hline YEAR & 2000 & 2030 & 2035 & 2040 \\
\hline TOTAL GNE & 576 & 1104 & 1175 & 987 \\
\hline PERCENT IN DISPERSED AREA & 100 & 100 & $100^{\circ}$ & 100 \\
\hline PERCENT EACH REACTOR & & & & \\
\hline LWR-U5(LE)/U & 75 & 7 & 4 & 3 \\
\hline LWR-US(DE)/U/TH & 24 & 37 & 28 & 22 \\
\hline SSCR-U3(DE)/U/TH & 1 & 48 & 52 & 53 \\
\hline SSCR-US(LE)/U & - & 8 & 16 & 23 \\
\hline
\end{tabular}




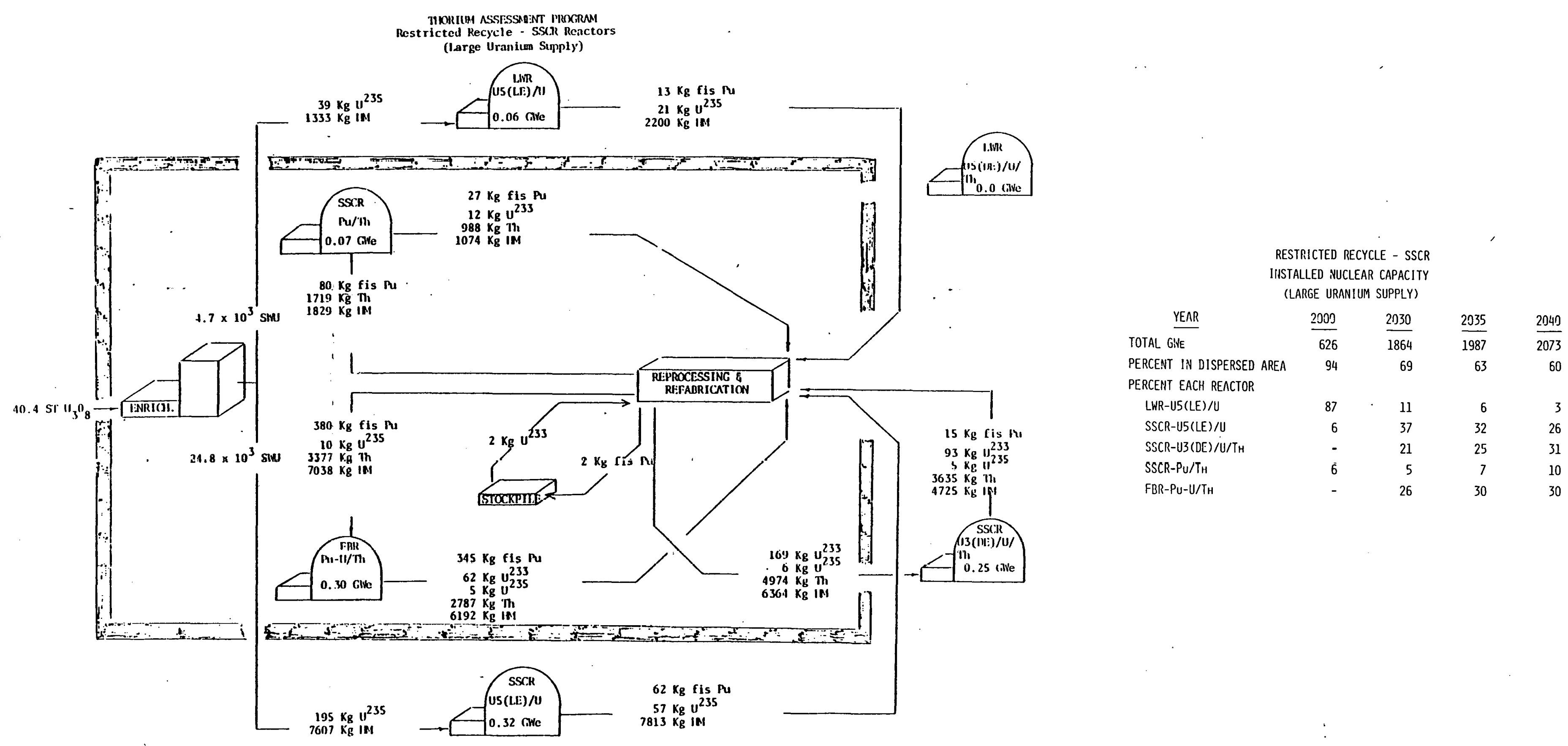




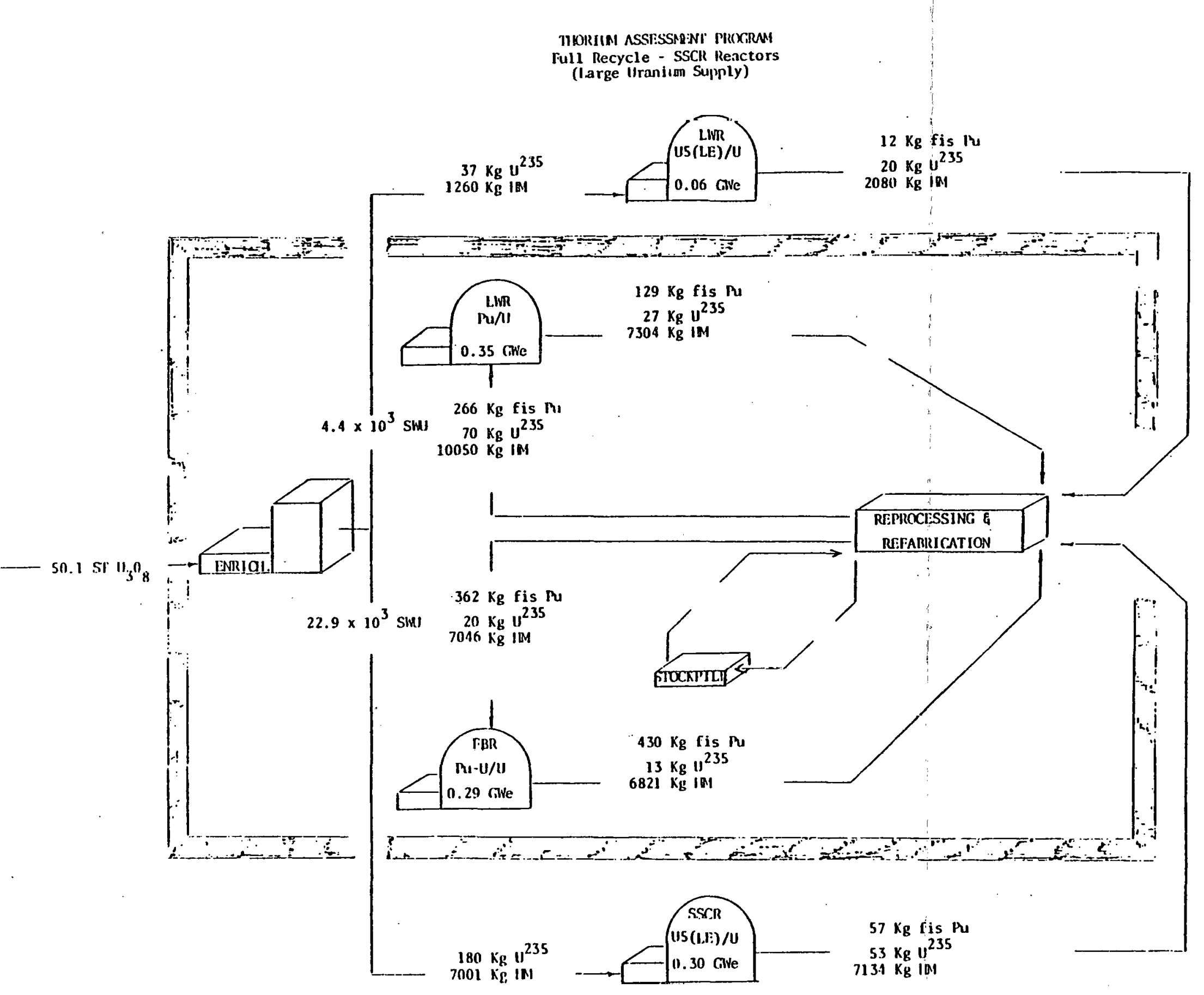

\begin{tabular}{|c|c|c|c|c|}
\hline \multicolumn{5}{|c|}{$\begin{array}{l}\text { FULL RECYCLE - SSCR } \\
\text { INSTALLED RUCLEAR CAPACITY } \\
\text { (LARGE URANIUM SUPPLY) }\end{array}$} \\
\hline YEAR & 2000 & 2030 & 2035 & 2040 \\
\hline TOTAL GiNE & 543 & 1453 & 196!: & 1556 \\
\hline PERCENT IN DISPERSED AREA & 94 & 44 & 36 & 28 \\
\hline \multicolumn{5}{|l|}{ PERCENT EACH REACTOR } \\
\hline LWR-U5(LE)/U & 88 & 10 & 6 & 3 \\
\hline LWR-Pu/U & 6 & 27 & 35 & 43 \\
\hline SSCR-US(LE)/U & 7 & 34 & 30 & 25 \\
\hline FBR-Pu-U/U & - & 29 & 29 & 29 \\
\hline
\end{tabular}


98
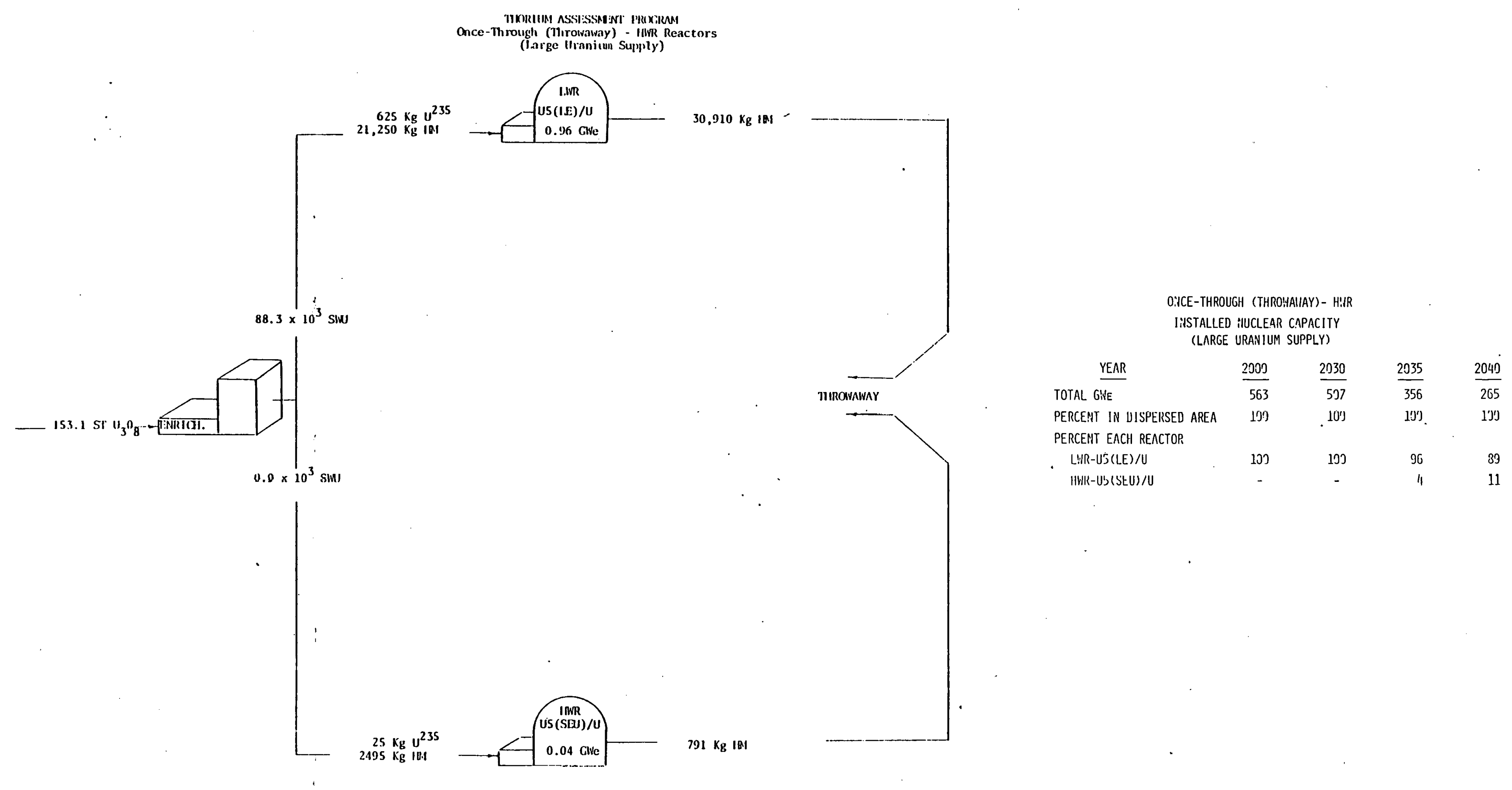


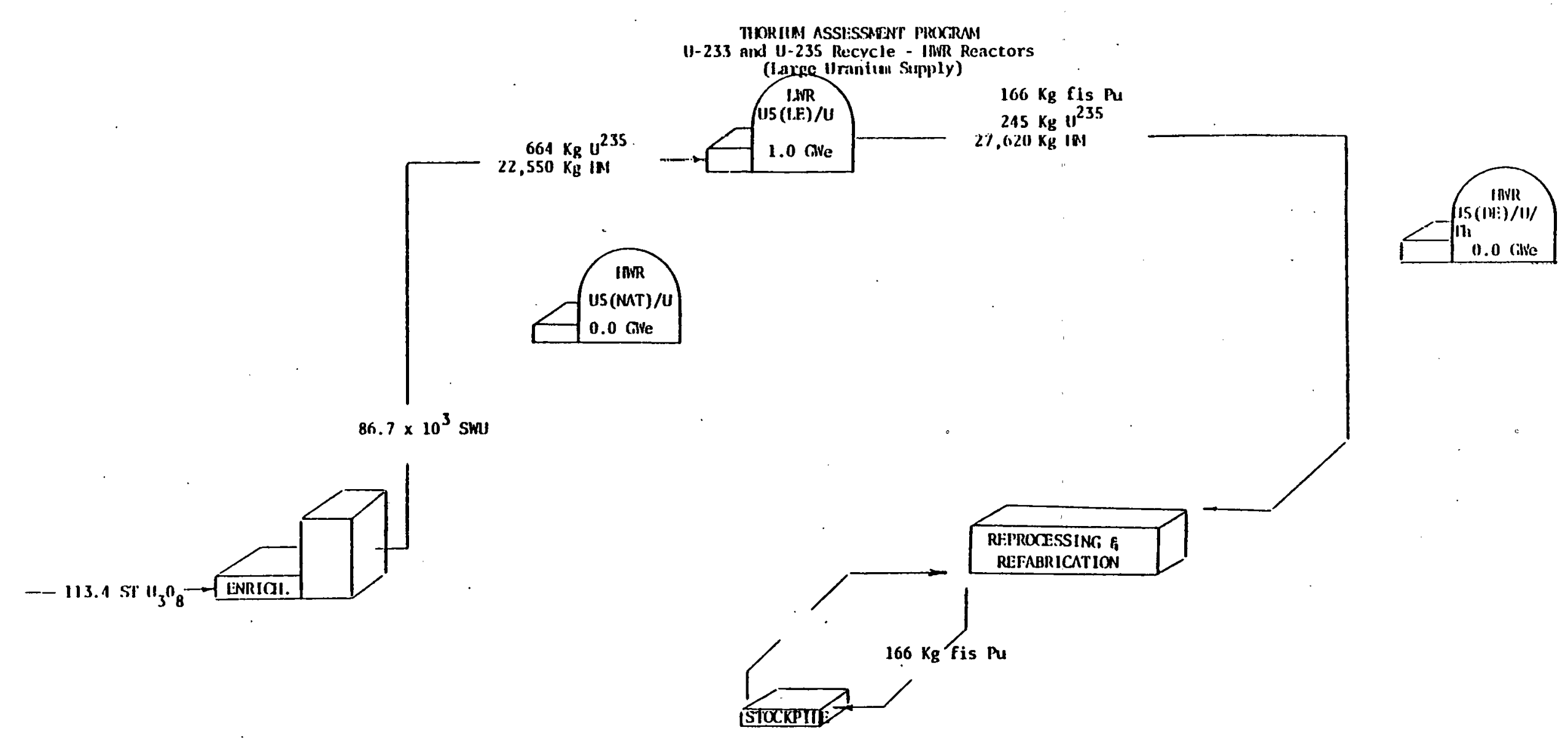

\begin{tabular}{|c|c|c|c|c|}
\hline \multicolumn{5}{|c|}{$\begin{array}{l}\text { U-233 A:DD U-235 RECYCLE - HUR } \\
\text { INSTALED NUCLEAR CAPACITY } \\
\text { (LARGE URANIUM SUPPLY) }\end{array}$} \\
\hline YEAR & 2003 & 2030 & 2035 & 2040 \\
\hline TOTAL GWE & 573 & $\overline{791}$ & $\overline{635}$ & $\overline{456}$ \\
\hline $\begin{array}{l}\text { PERCENT IN DISPERSED AREA } \\
\text { PERCENT EACH REACTOR }\end{array}$ & 100 & 100 & 100 & 100 \\
\hline$L W R-U 5$ (LE)/U & 100 & 100 & 100 & 100 \\
\hline
\end{tabular}

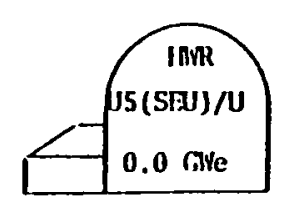




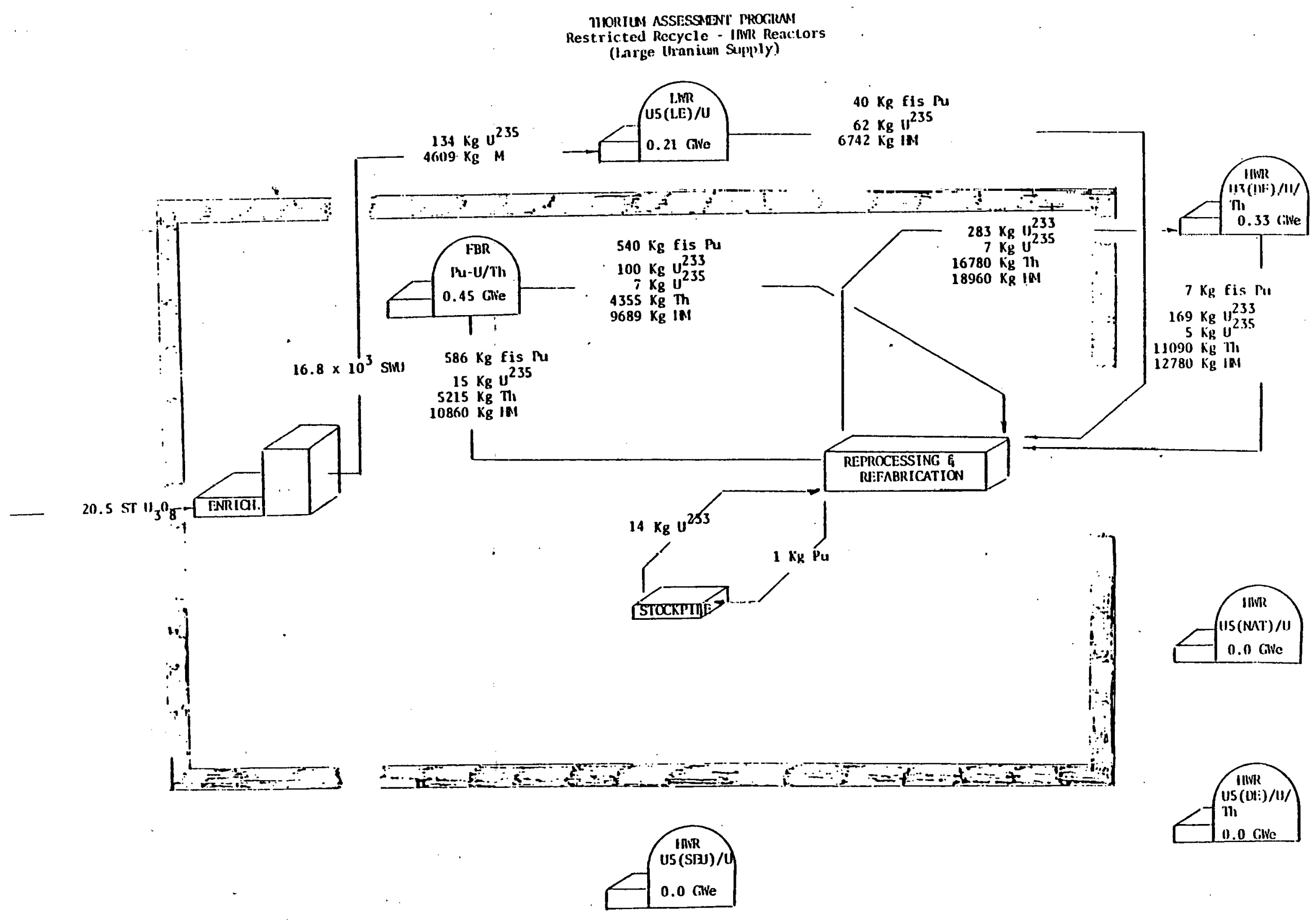

\begin{tabular}{|c|c|c|c|c|}
\hline \multicolumn{5}{|c|}{$\begin{array}{l}\text { RESTRICTED RECYCLE - HWR } \\
\text { IWSTALLED NUCLEAR CAPACITY } \\
\text { (LARGE URANIUM SUPPLY) }\end{array}$} \\
\hline YEAR & 2009 & 2030 & 2035 & 2040 \\
\hline TOTAL GNE. & $\overline{634}$ & $\overline{1802}$ & 2008 & 2187 \\
\hline PERCENT IN DISPERSED AREA & 100 & 56 & 55 & 53 \\
\hline PERCENT EACH REACTOR & & & & \\
\hline LWR-U5(LE)/U & 100 & 35 & 21 & 12 \\
\hline HWR-U3(DE)/U/TH & - & 21 & 33 & 42 \\
\hline FRR-Pu-II/TH & - & 44 & 45 & 47 \\
\hline
\end{tabular}


101

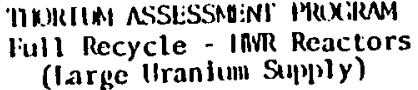
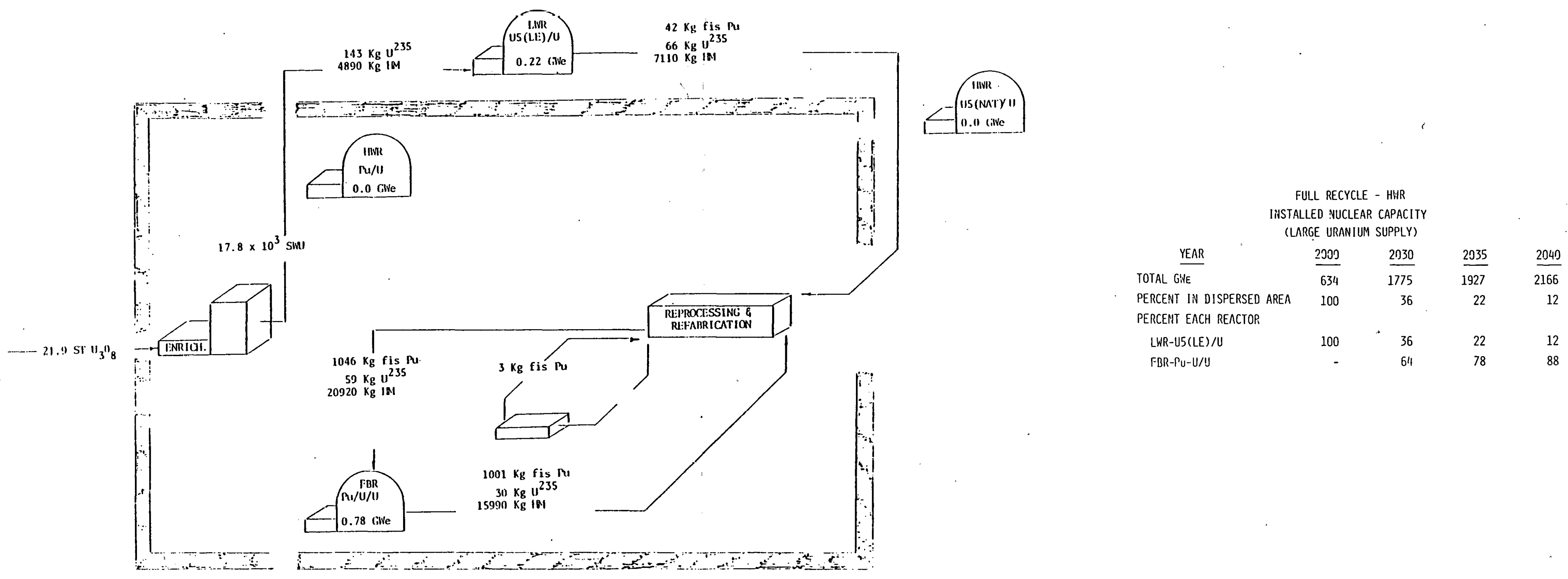

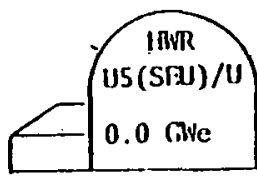



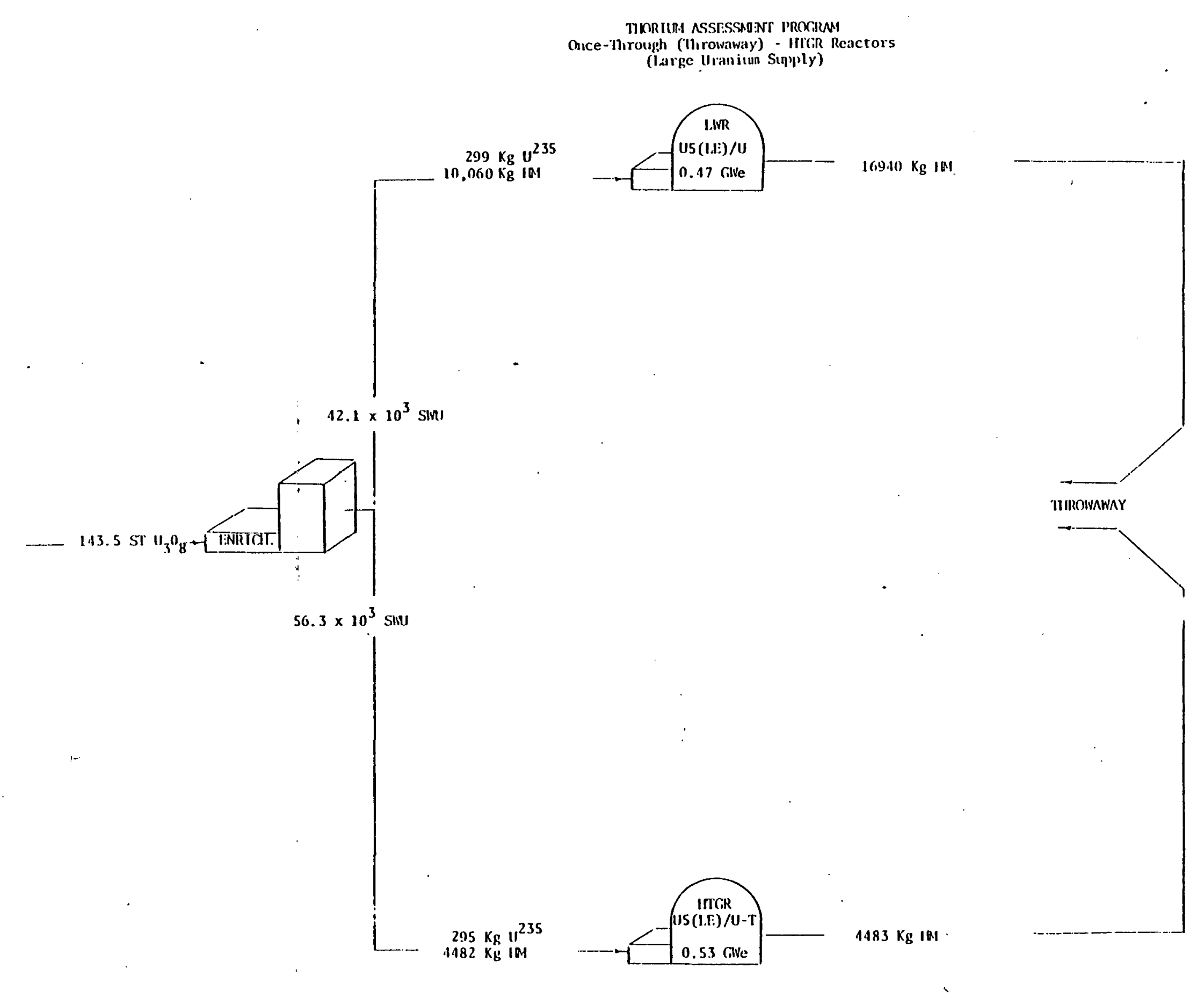

ONCE-THROUGH (THROHANAY) - HTGR IIISTALLED NUCLEAR CAPACITY (LARGE URANIUM SUPPLY)

\begin{tabular}{|c|c|c|c|c|}
\hline YEAR & 2000 & 2030 & 2035 & 2040 \\
\hline TOTAL GNE & 563 & 556 & 409 & 318 \\
\hline PERCENT IN DISPERSED AREA & 100 & 100 & 100 & 100 \\
\hline \multicolumn{5}{|l|}{ PERCENT EACH RENCTOR } \\
\hline LWR-US(LE)/U & 100 & 59 & 47 & 36 \\
\hline$\| I T G R-U 5(L E) / U-T$ & - & 41 & 53 & 64 \\
\hline
\end{tabular}




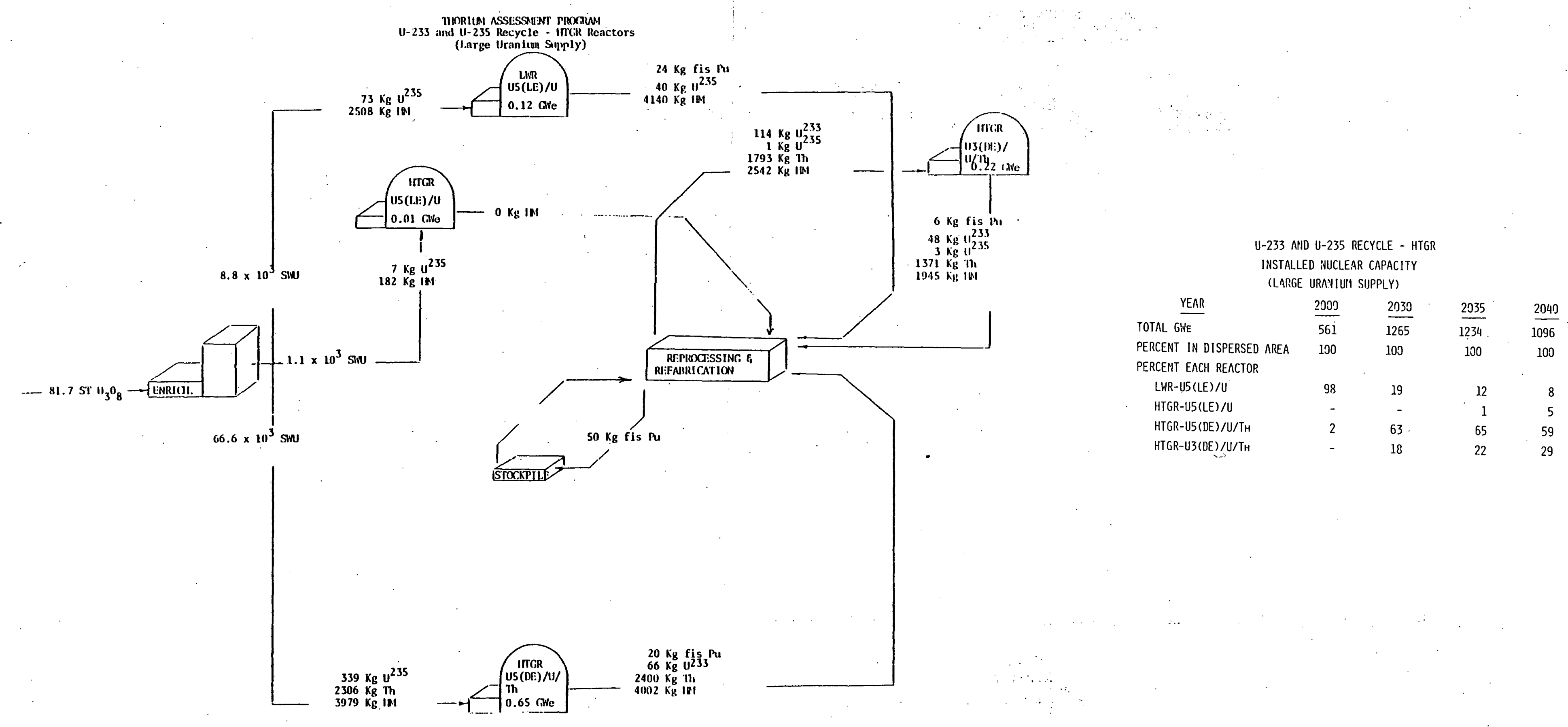




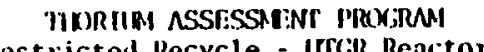

(larpe Uriulien Stuly

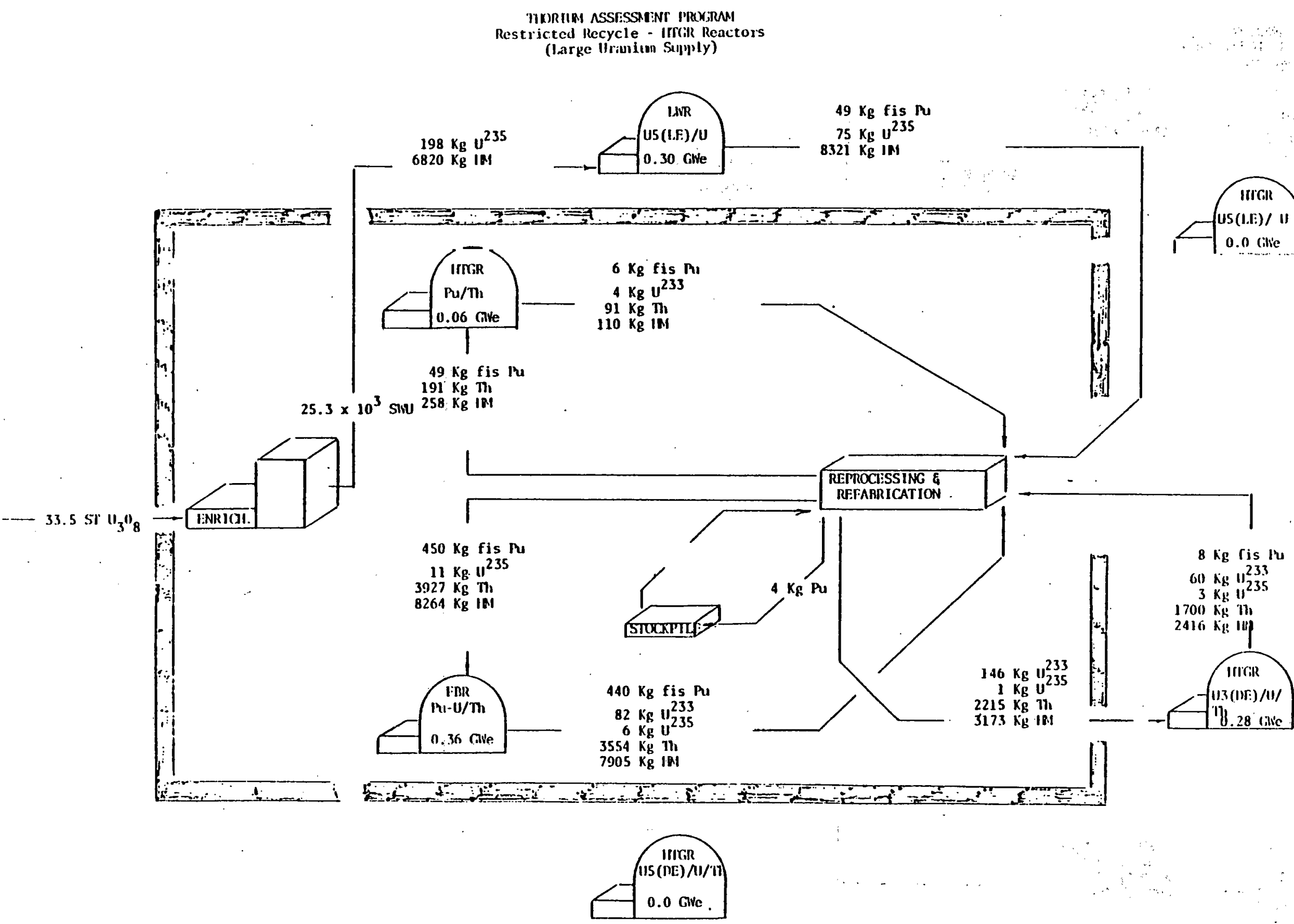

RESTRICTED RECYCLE - HIGR WSTALLED NUCLEAR CAPACIT (ARGE LRAAIIUI SUPPLY) TOTAL GNE PERCENT IN DISPERSED MOEA 634 PERCENT EACH REACTOR LWR-U5(LE)/U

HTGR-U3 (DE)/U/T

HTGR-PU/TH

FBR-PU-U/TH 


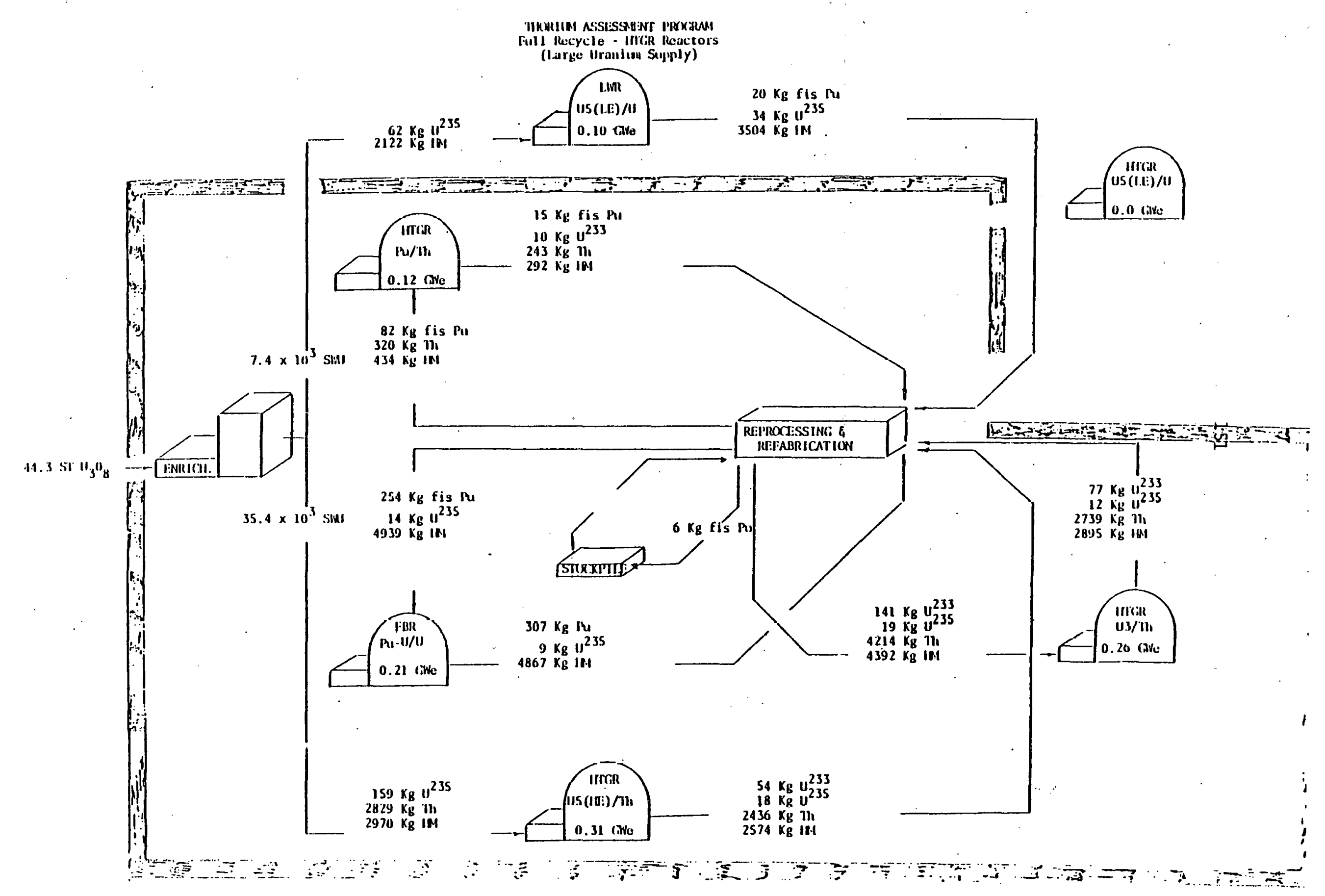

\begin{tabular}{|c|c|c|c|c|}
\hline $\begin{array}{r}F \\
\text { lisTA } \\
\text { CLAR }\end{array}$ & $\begin{array}{l}\text { RECYC } \\
\text { ED HUCL } \\
\text { URAIIU }\end{array}$ & $\begin{array}{l}\text { HTGR } \\
\text { APACITY } \\
\text { PLY) }\end{array}$ & & \\
\hline YEAR & 2000 & 2030 & 2035 & 2040 \\
\hline TOTAL GWE & $\overline{626}$ & $\overline{1903}$ & 2046 & 2140 \\
\hline $\begin{array}{l}\text { PERCENT IN DISPERSED AREA } \\
\text { PERCENT EACH REACTOR }\end{array}$ & 98 & 18 & 10 & 5 \\
\hline LWR-U5(LE)/U & 98 & 18 & 10 & \\
\hline HTGR-US(HE)/TH & 1 & 31 & 31 & 29 \\
\hline HTGR-U3/TH & - & 20 & 26 & 32 \\
\hline HTGR-Pu/TH & 1 & 11 & 12 & \\
\hline FBR-PU-U/U & & 21 & 21 & 19 \\
\hline
\end{tabular}




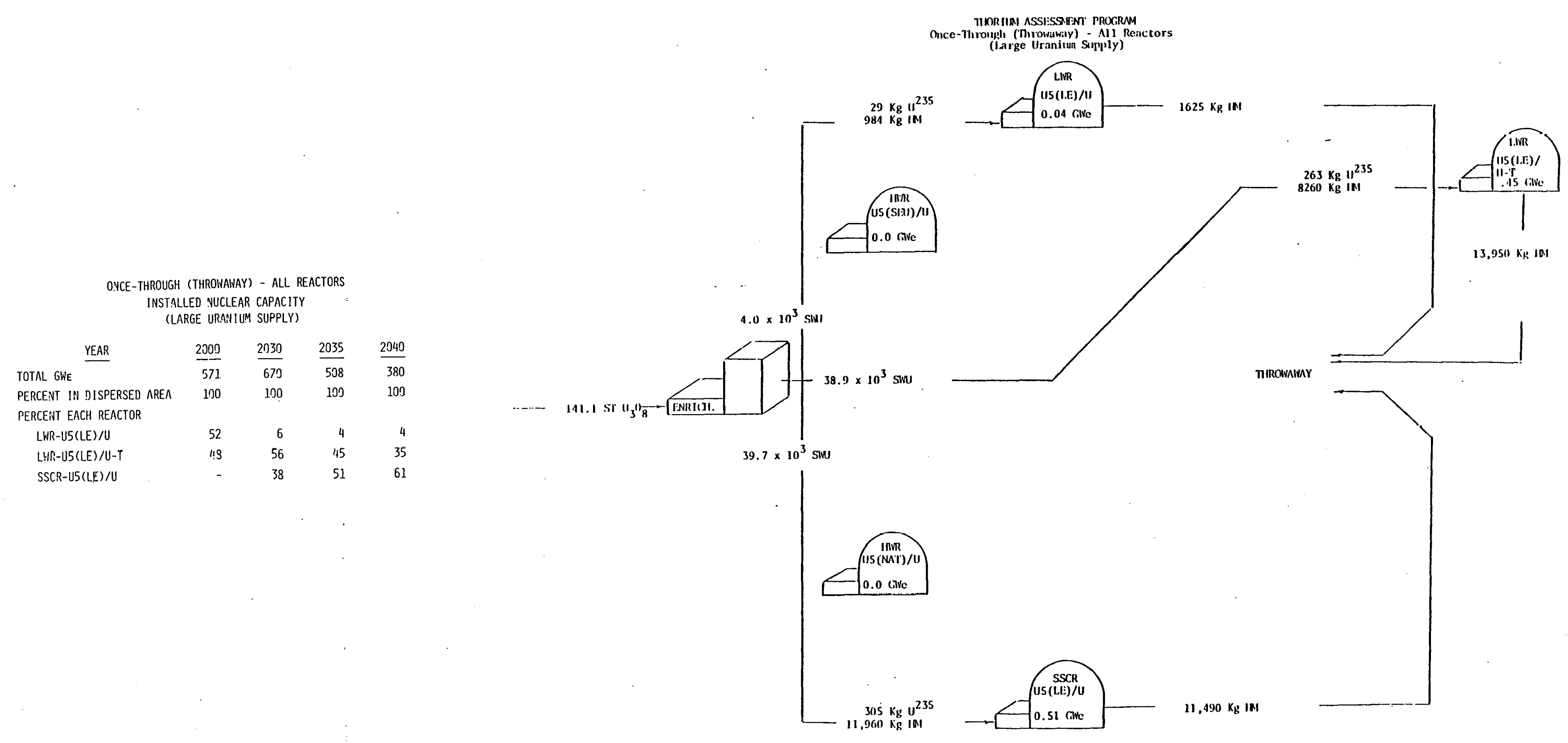




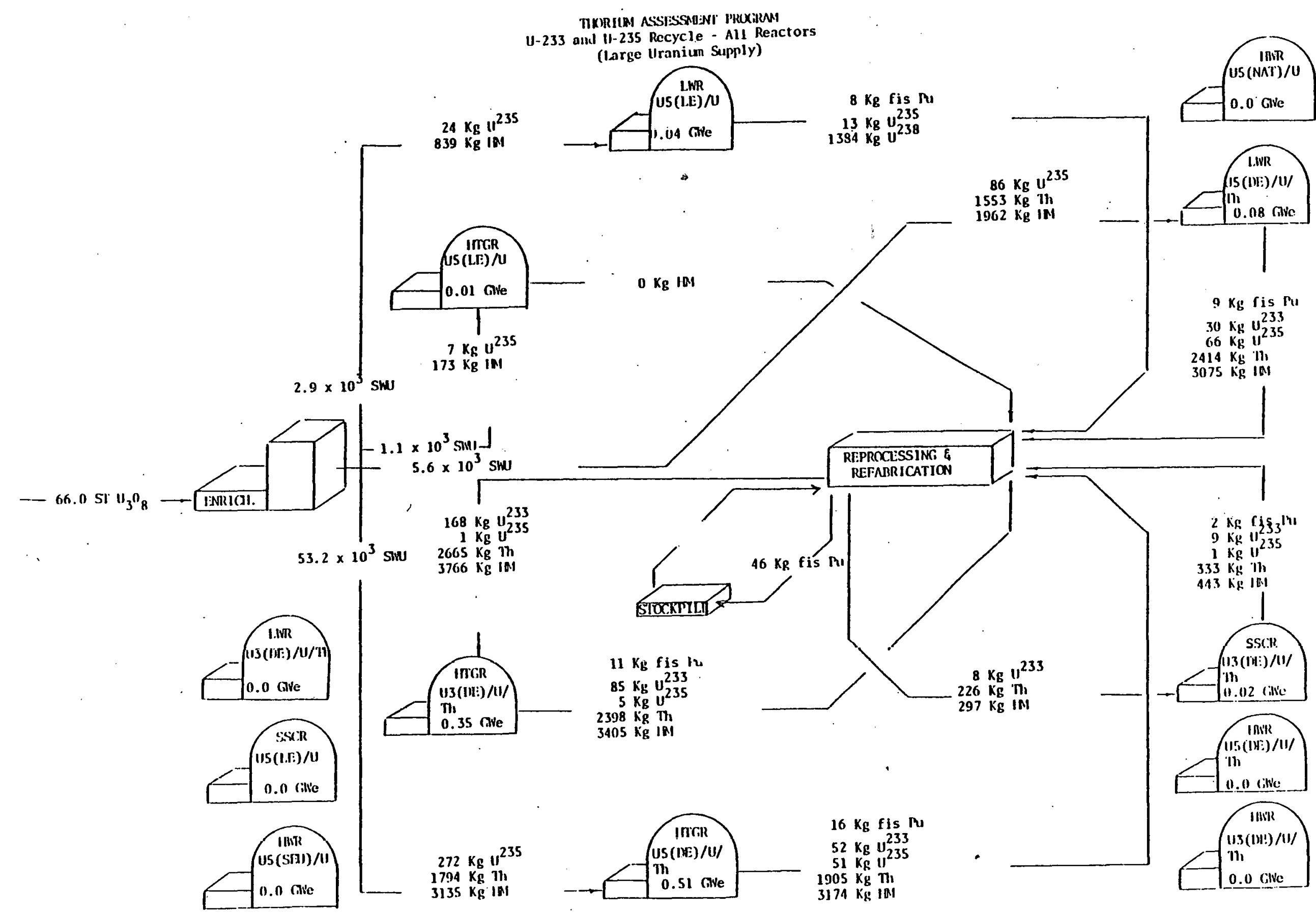

\begin{tabular}{|c|c|c|c|c|}
\hline \multicolumn{5}{|c|}{$\begin{array}{l}\text { U-233 Aid U-235 RECYCLE - ALL REACTORS } \\
\text { I:ISTALLED NUCLEAR CAPACITY } \\
\text { (LARGE URANIUM SUPPLY). }\end{array}$} \\
\hline YEAR & 2000 & 2030 & 2035 & 20 \\
\hline TOTAL GWE & 561 & 1359 & 1285 & 11 \\
\hline $\begin{array}{l}\text { PERCENT IN DISPERSED AREA } \\
\text { PERCENT EACH RENCTOR }\end{array}$ & 100 & 100 & 100 & \\
\hline $\begin{array}{l}\text { ERCENT ENCH RENCTOR } \\
\text { LWR-U5(LE)/U }\end{array}$ & 77 & 6 & 4 & \\
\hline LWR-US (DE)/U/TH & 20 & 13 & 8 & \\
\hline $\operatorname{SSCR}-\mathrm{U} 3(\mathrm{DE}) / \mathrm{U} / \mathrm{TH}_{\mathrm{H}}$ & - & 2 & 1 & \\
\hline HTGR-U5(LE)/U & - & - & 1 & \\
\hline HTGR-U5(DE)/U/TH & 2 & 47 & 51 & \\
\hline HTGR-U3(DE)/U/TH & 1 & 31 & 35 & \\
\hline
\end{tabular}




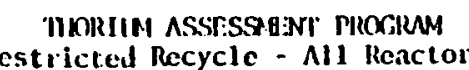

(larye Urwiim Supply)

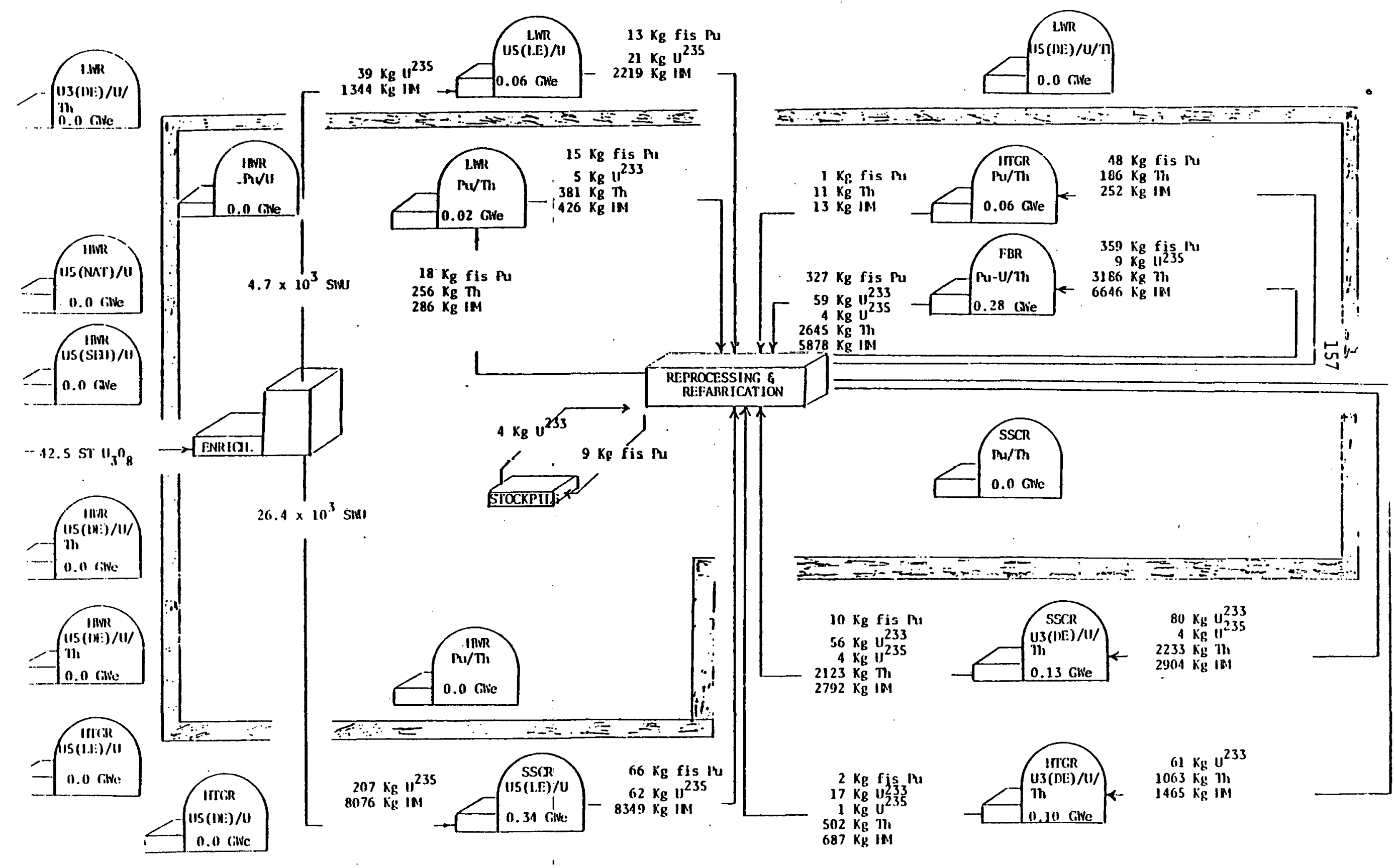

\begin{tabular}{|c|c|c|c|c|}
\hline \multicolumn{5}{|c|}{$\begin{array}{l}\text { RESTRICTED RECYCLE - ALL IEEACTORS } \\
\text { I.SSTALLED VUCLEAR CAPACITY } \\
\text { (LARGE URNIUM SUPPLY) }\end{array}$} \\
\hline YEAR & 2000 & 2030 & 2035 & 2040 \\
\hline TOTAL GWE & $\overline{626}$ & $\overline{1615}$ & 2000 & 2977 \\
\hline PERCENT IN DISPERSED ARE & 91 & 70 & 63 & 58 \\
\hline \multicolumn{5}{|l|}{$\begin{array}{l}\text { PERCENT EACH REACTOR } \\
\text {. }\end{array}$} \\
\hline LWR-US(LE)/U & 84 & 11 & 6 & 4 \\
\hline LWR-Pu/TH & 5 & 3 & 2 & 1 \\
\hline SSCR-U5(LE)/U & b & 39 & 34 & 28 \\
\hline SSCR-U3(DE)/U/TH & 1 & 14 & 13 & 11 \\
\hline 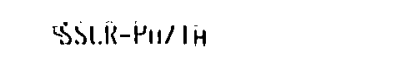 & 3 & 1 & & \\
\hline HTGR-U3(DE)/U/TH & - & 6 & 10 & 16 \\
\hline Hї̈K-P̈U//H & 1 & 1 & 6 & 11 \\
\hline FBR-PU-U/TH & - & 25 & 28 & 28 \\
\hline
\end{tabular}




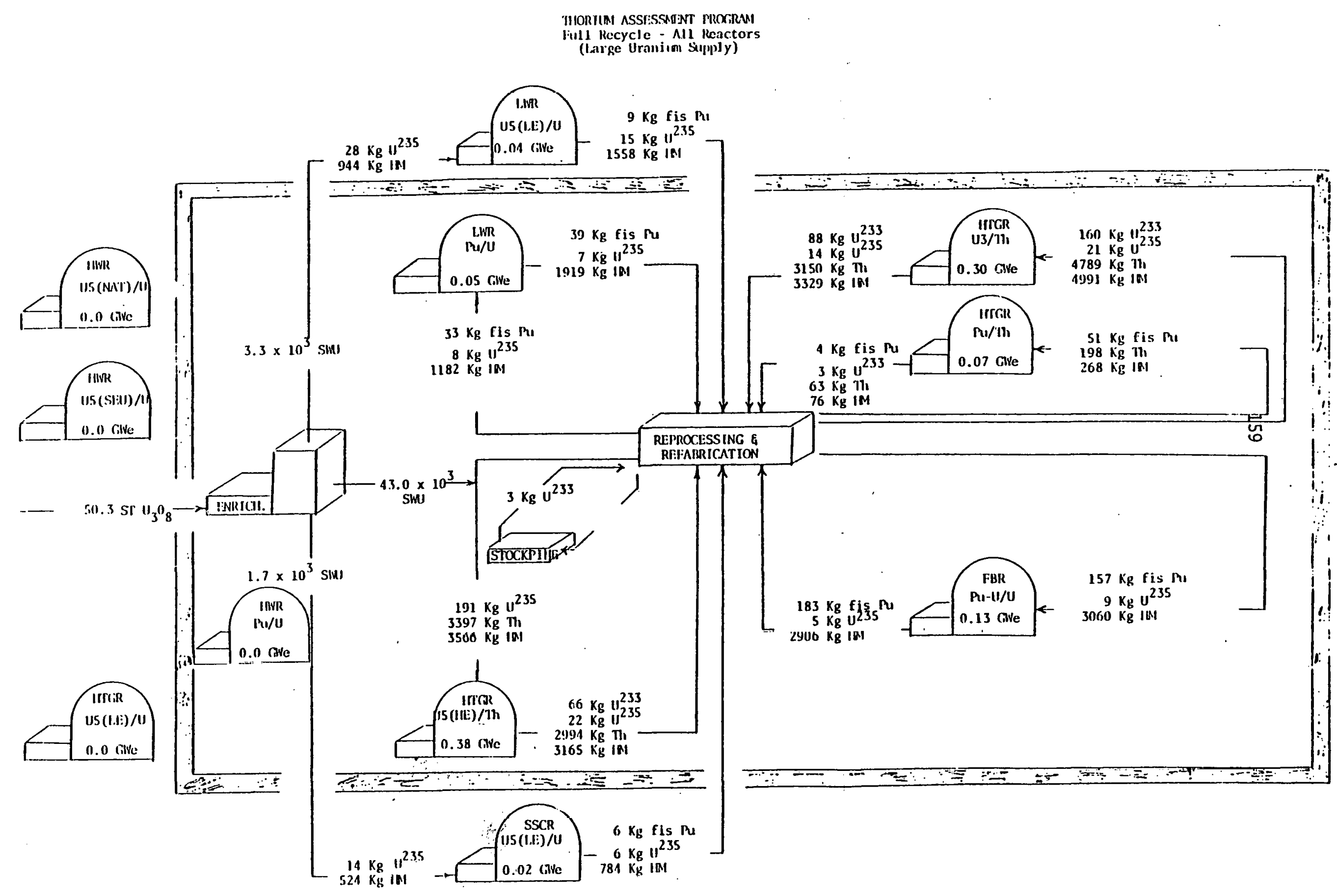

\begin{tabular}{|c|c|c|c|c|}
\hline \multicolumn{5}{|c|}{$\begin{array}{l}\text { FULL RECYCLE - ALL REACTORS } \\
\text { IHSTALLED NUCLEAR CAPACITY } \\
\text { (LARGE URANIUM SUPPLY) }\end{array}$} \\
\hline YEAR & 2900 & 2030 & 2035 & 2040 \\
\hline TOTAL GWE & 620 & $\overline{1923}$ & $\overline{2065}$ & 2127 \\
\hline PERCENT IN DISPERSED AREA & 91 & 12 & 6 & 3 \\
\hline \multicolumn{5}{|l|}{ PERCENT EACH REACTOR } \\
\hline LWR-US(LE)/U & 85 & 8 & 4 & 2 \\
\hline LWR-Pu/U & 6 & 10 & 5 & 3 \\
\hline SSCR-US(LE)/U & 6 & 4 & 2 & 1 \\
\hline HTGR-U5(HE)/TH & 2 & 38 & 38 & 35 \\
\hline HTGR-U3/TH & - & 23 & 30 & 37 \\
\hline HTGR-PU/TH & 2 & 4 & 7 & 9 \\
\hline FBR-Pu-U/U & - & 13 & 13 & 12 \\
\hline
\end{tabular}


THIS PAGE

WAS INTENTIONALLY

LEFT BLANK 


\section{ANNEX 2}

TECHNICAL ASPECTS OF THORIUM UTILIZATION

$$
\begin{aligned}
& \text { Norton Ly Shapiro } \\
& \text { Combustion Engineering; Inc: }
\end{aligned}
$$




\section{THIS PAGE}

\section{WAS INTENTIONALLY LEFT BLANK}


TECHNICAL ASPECTS OF THORIUNA UTILIZATION

\section{$2.1 \quad$ IIITROOUCTION}

The possibility of utilizing thorium as a fuel for nuclear reactors has been recognized since 1946 when it was discovered that Th-232 could be transmuted into the fissile isotope U-233, and interest in utilizing thoriumbased fuels in thermal reactors has persisted over the years because of the superior nuclear characteristics of U-233. Al though thorium fuels have been employed in experimental and demonstration programs, large-scale commercial utilization of thorium has not evolved for a number of reasons. The U.S. Nuclear Power Development Program, from its inception, has been predicated on the development of breeder reactors toward the latter part of the 20th century. Thermal reactors, such as those currentiy employed throughout the U.S., were viewed as an interim source of electrical power and as a means of developing the technology and of generating the fissile material required for the eventual deployment of the fast breeder reactor. The superior performance in fast reactors of the plutonium hred from uranium fuels, and the more extensive development of the uranium fuel cycle for military applications led to a decision to develop nuclear reactors based on the uranium fuel cycle. Subsequent assessments of thorium utilization, performed in the 60s, identified a number of incentives for thorium utilization, but concluded that the uranium cycle would be more economical, under the economic conditions of the time, for the light-water-moderated reactors (LWR) being deployed.

A number of economic and political changes have occurred since the decision was initially made to develop nuclear reactors based on uranium fuels and since the last comprehensive review of thorium utilization was performed by the AEC in 1969. Foremost of these has been the developing concern of the proliferation of nuclear weapons, which has resulted in a decision to postpone indefinitely plutoniun utilization and the deployment of the plutoniumfueled fast breeder reactor in the U.S., and to encourage other nations to adopt a similar policy. As a result of this decision, thermal converter 
reactors will have to play a more significant and longer-term role in the generation of nuclear power than had previously been anticipated, thus placing an increased emphasis on the potential of alternate fuel cycles for uranium resource conservation: The changed economic climate, in particular the significant increase in uranium price over the past few years, has also increased the incentive to consider the development of alternatives, such as thorium fueling, which reduce uranium demand. In this chapter, the technical, economic and resource utilization aspects of thorium utilization are reviewed to establish the advantages and disadvantages of thorium utilization with respect to the changed political and economic considerations mentioned above.

\subsection{RESULTS OF PREVIOUS STUDIES}

An examination of the most recent comprehensive assessment of thorium utilization performed by the AEC in June 1969, WIASH $1097^{1}$, hightights some of the reasons why thorium has not received a high level of support for commercial development. An essential ground rule used in this earlier ieport is a supposition that the primary incentive for the development of nuclear power was economic--more specifically, the reduction in the cost of power. Whether a specific reactor uses a thorium or uranium cycle was viewed to depend upon the expected economics of the respective cycles for the applicable financial and technological conditions, and on the impact of the specific fuel strategies selected upon the overall electric system economics. The report did consider the efficiency of natural resource utilization, that is, how much electrical power can be produced from a given ton of mined uranium ore, but the key conclusions with respect to the potential for commercial deployment were primarily based on the economic incentive. Key conclusions from this report are summarized briefly below.

1. The possibility of utilizing thorium fueling in the light-liatermoderated reactor (LWR), the heavy-water-moderated reactor (HWR), the high-temperature gas-cooled reactor (HTGR), the molten salt breeder reactor (IISBR), and the fast-breeder reactor (FGR) were assessed with the conclusion that thorium fueling could be used in each type. 
2. Under the economic ground rules employed in the study (e.g., $\$ 8 / 1 \mathrm{~b}$ $\mathrm{U}_{3} \mathrm{O}_{8}$ ), the uranium cycle was viewed to be more economical than the thorium cycle in the L!IR or HWIR.

3. The Indian Point PUR was operated initially with U-235 enriched thorium fuel. This plant successfully demonstrated the possibility of using the thorium fuel cycle in an. LVR, as well as the ability of converting completelly: to the uranium fuel cycle in the same plant. (It should be noted, however, that irradiation demonstrations of the performance of thorium-based fuels in fuel rods of modern design will probably also be required). The report concluded, however, that there was no economic motivation to pursue the thorium cycle in presently-developed water reactors unless economic factors changed significantly from those prevalent at the time of the study.

4. The use of heavy water as a moderator permits the use of natural or slightly-enriched uranium as fuel. The resulting fuel inventory and make-up cost with the uranium cycle are so low that very large changes in the uranium ore cost were judged necessary to make the thorium cycle economically competitive.

5. The use of thorium fueling in such reactors as the high-temperature gas-cooled reactor (HTGR) and the molten-salt-breeder reactor (MSBR) appear to be more economic. Although more of the expensive, highly enriched $U-235$ is used, the increased fissile inventory cost is more than compensated by the savings in fuel depletion cost achieved with the thorium fuel cycle due to the higher fuel conversion ratio. In the future (after about the late 70s), the use of thorium in the HTGR indicated a potential levelized fuel cycle cost savings of up to $0.4 \mathrm{mils} / \mathrm{kwhr}$, and in the more distant future (after about 1985) the use of the thorium cycle in the MSBR indicated potential fuel cycle cost savings of up to $1.0 \mathrm{mils} / \mathrm{kwhr}$ over those obtainable with LWRs operating on either the thorium or uranium cycles. 
6. Since the fuel inventory costs of reactors using the thorium cycle are higher than those of reactors using the uranium cycle, high interest rates on the fuel inventory penalizes the thorium cycle more than uraniuri; conversely, lower interest rates favor the thorium cycle. Future increases in uranium ore cost and/or decreases in the cost of enrichment tend to favor the thorium cycle relative to the use of the uranium cycle.

7. Because thorium does not contain any natural occurring fissile material, $U-235$ is required for initial inventory and burnup, and therefore the requirements for both uranium and thorium ores must be evaluated when considering the use of the thiorium fuel cycle.

8. The economic development of the thorium fuel cycle will significantiy increase our economically exploitable resources of nuclear fuel. Although there is little incentive to develop the thorium fuel cycle solely to increase the supply of fertile material (because of the abundarice of the fertile material $U-238)$, the higher conversion ratios which can be obtained in thermal spectrum reactors using the bred $11-233$ instead of Pu- 239, can result in significantly better utilization of natural uranium fuel resources in thorium-fueled reactors than with low-enrichment uranium fuel.

9. The economic utilization of nuclear resources is not necessarily consistent with optimal conservation of these resources.

Overall, WASH-1097 concluded in 1969 that al though the thorium fuel cycle had certain, inherent technical features, such as improved fuel utilization in thermal spectrum reactors, the fuel cycle economics were not sufficiently favorable to justify the commercial development of the thorium cycle in the U.S. at that time, at least for LWRs and FBRS. The report pointed out that, in general, various applications of the thorium fuel cycle appeared technically feasible, although some additional R\&D would be required. Noting that the LURR and HTGR applications had been demonstrated, the 
report concluded that technical feasibility would not prevent commercial deployment of the fuel cycle, at least in these reactor applications.

In addition to the WASH-1097 study, numerous other evaluations of thorium have been made over the past $10-15$ years. A bibliography of the most relevant of these is provided.*.

\subsection{THE MEED FOR A REVISED ASSESSHENT OF THE THORIUM CYCLE}

Although the conclusions reached in the 1969 WASH-1097 study, and in other previous evaluations, are generally valid today, a number of political and economic changes have evolved since the time of this study which necessitate a careful reevaluation of the incentives for thorium utilization. These current-day factors are discussed briefly below.

1. Conclusions as to the desirability of implementing the thorium option. have, in the past, been viewed almost entirely within the context of the: economic competition with the uranium fuel cycle. At the time of these previous studies, nuclear power was striving to compete economically with the then inexpensive fossil-fuel alternatives, and the deployment of al ternate fuel cycles which might lead to a competitive disadvantage for nuclear power was unattractive. Although the cost of uranium ore and enrichment services has increased over the years, the cost of al ternate energy sources has increased even more rapidiy so that nuclear. power now enjoys a significant cost advantage in most areas of the nation. Fuel cycle economics, of course, remains an. important consideration in the selection of fueling options; in the absence of other mitigating considerations, the most economical alternative would undoubtedly be selected. However, alternatives such as thorium fueling can at least now be considered even though they may not represent the economic optimum alternative provided they contribute in other ways to the energy goals of the nation without seriously compromising the economics of nuclear power with respect to other alternatives. Such goals as a reduction in the potential for nuclear weapons

* See Append1x A, Volume I, 
proliferation and the conservation of uranium resources are consideiations which are not necessarily reflected in the economic intercomparison of alternate fuel cycles.

2. At the time of previous studies, the breeder reactor, with the promise of inexpensive and essentially resource unlimited power, was perceived to be realizable in the not too distant future, and hence the uranium. conservation aspects of al ternate fuel cycles were of less importance. Relatively recentiy, concerns for the potential for nuclear weapons proliferation have received prominence, and have resulted in President Carter's April 7, 1977 decision to defer cormercial reprocessing and the deploynent of the plutonium-fueled LMFBR. The deferment of the LMFBR Program has contributed to growing concerns over the adequacy of uranium resources and production capacity, and has heightened interest in a?ternate fuel cycle and reactor concepts which might extend uranium resources in the absence of an LMFBR, or for use in conjunction with modified LMFBR concepts.

3. Since 1969, the price of uranium ore has risen from $\$ 8 / 16$ to current prices of $\$ 30 / 16$ or more. Although the price of uranium in the near term is difficult to predict, since it is subject to supply and demand considerations, the long-term trend should be for uranium prices to increase, reflecting the increasing scarcity of uranium as more economically recoverable deposits are consumed. Increases in uranium price will tend to make the thorium cycle more competitive with respect to uranium fueling. The cost of other fuel cycle components, such as the cost of enrichment services, have also increased and these increases will tend to reduce the incentive for thorium utilization; however, a number of technological innovations, such as centrifugal and eventually laser enrichment, are foreseen, which might provide further economic incentive for thorium fueling by reducing the cost of separative work. At any rate, it is clear that a number of economic factors have changed so significantly from those employed in the WASH-1097 evaluation, that a reevaluation of the economic incentives for thorium utilization is required. 
4. As mentioned above, many of the current investigations of alternate fuel cycles have been motivated by considerations of nuclear weapons proliferation. Central to most proliferation resistant fuel cycle. alternatives is the reduction or elimination of plutonium utilization in nonweapons states. Although the once-through cycle which is currently employed achieves this objective, it is gained at the expense of uranium resource conservation and of an assured long-term fissile fuel supply. Certain thorium fuel cycles offer the potential of a more proliferationresistant fuel.cycle while providing an appreciably more efficient resource utilization. This incentive for the thorium fuel cycle is dis-. cussed in more detail in Section 2.4.

5. At the time the initial thorium utilization assessments were performed, the nuclear capacity on line, under construction, or committed was very sma17. The HASH-1097 analysis could then consider in an even-handed way a variety of alternate fuel cycles and reactor types, since the future U.S. nuclear economy was not committed to any particular path. Today, however, more than 45 Gwe of LHR capacity is in operation in the U.S. and nore than 200 Gwe of LIIRs are committed by 1990. Consequently, it is clear that the characteristics of the overall vs. nuclear economy will be dominated by the performance of the LWR until well into the 21 st century. Consequently, an analys is of the impact of alternate fuel cycles or reactor types must consist of a systems analys is in which the existing 'and planned LUR capacity is represented along with any modified fuel cycles or reactor types contemplated for future deployment. Since a substantial fraction of our potential uranium resources will be required to fuel these LWRS, assessments performed today must address such questions as whether the remaining uranium base. is sufficiently large to make worthwhile the deployment of alternate reactor types or fucl cycles, and questions of whether more efficient thorium fuel cycles can be backfit into planned LIIR capacity. 


\subsection{FEATURES CF THE THORIU:A CYCLE}

\subsubsection{Nuclear Characteristics}

Thorium is a natural occurring element which is about three times more abundant in the earth's crust than uranium. Thorium occurs in nature as essentially pure Th- 232 which, like $U-238$, is a fertile material. then irradiated by neutrons in a reactor. Th-232 is converted into a fissile isotope of uranium, U-233, in a manner analogous to the conversion of $U-238$ into Pu-239.

The isotopic buildup in thorium and uranium is compared in Figure 2.1. The capture of a neutron in Th-232 or U-238 leads, after the $B$ decay. of an intermediate isotope, to the production of a fissile isotope, U-233 in the case of thorium and Pu-239 in the case of uranium. Absorption of a neutron by these fissile isotopes leads either to fission or to the fertile isotopes U-234 or Pu-240 which in turn can be transmuted to the fissile isotopes U-235 or Pu-24l by the capture of an additional neutron. Neutron absorption in either of these fissile isotopes lead either to fission or to the production of parasitic neutron absorbers.

The U-235 component of uranium is the only fissile material present in any significant quantity in nature. Since natural thorium contains no fissile component comparable to U-235, the use of a thorium fuel cycle does not preclude the need for uranium. U-235 from uranium ore must either be used directly with thorium to start the cycle or indirectly in a uranium cycle to produce plutonium which, in turn, would provide the initial fissile component in a thorium cycle. For reactors other than the breeder reactor (converter reactors) annual additions of fissile material are required to supplement the fissile material bred during the cycle. Here again, U-235 obtained from uranium ore or plutonium produced in the uranium cycle is necessary for continued operation. In this sense, the thorium cycle is coupled to the uranium cycle and the availability of uranium ore.

As mentioned above, thorium consists of the fertile material Th-232 and consequently thorium is an alternative to the fertile component of uranium 


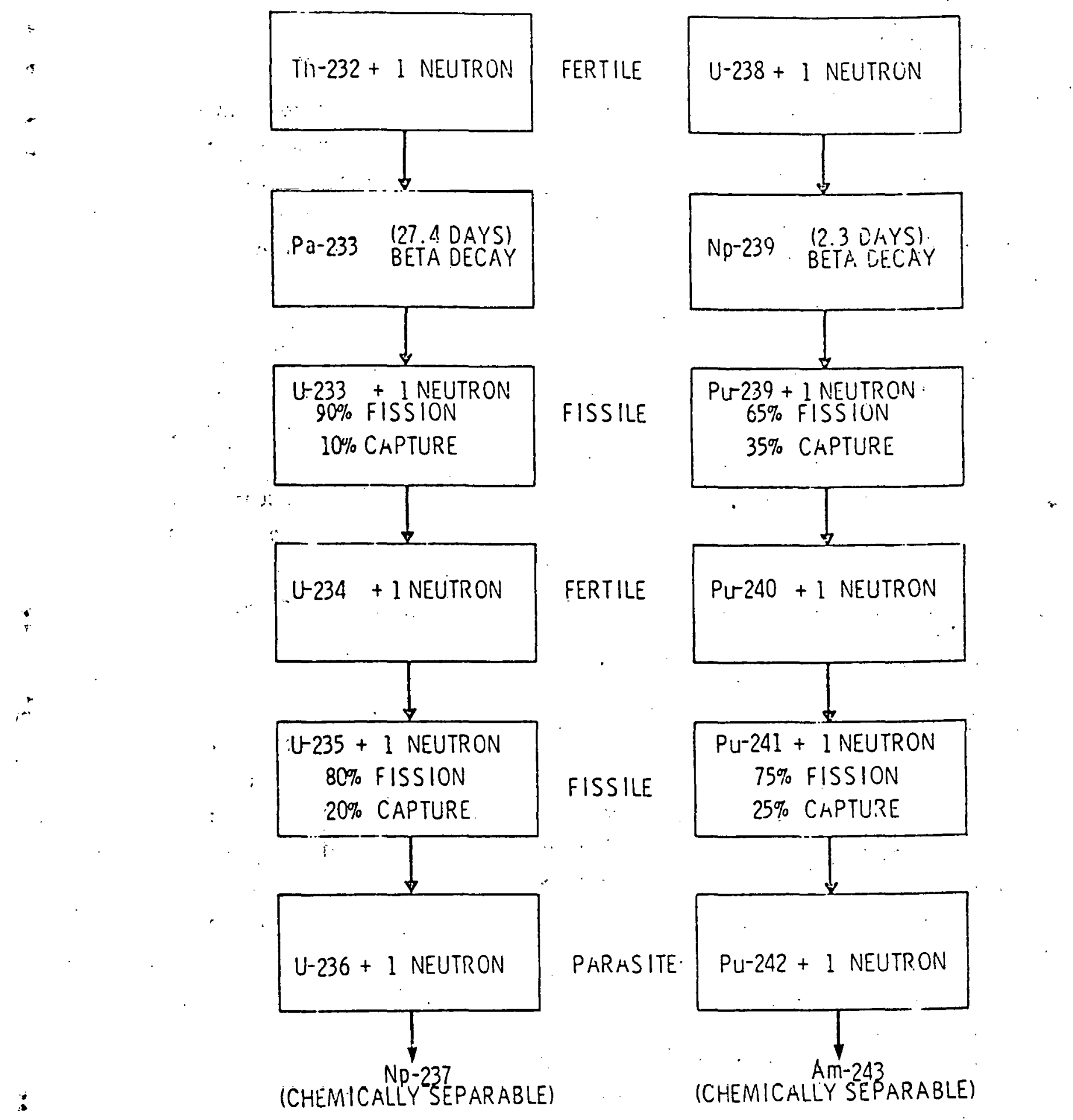

Figure 2.1 THE ISOTOPIC BUILDUP IN THORIUM AND URANILIM 
U-238, rather than a replacement for uranium ore itself. The large quantities of uranium required for our nuclear industry are not needed for the. U-238 content, but for the U-235 which is also present in small quantities in uranium ore. Since thorium, unlike uranium, does not contain a fissile component, thorium, per.se, adds nothing significant to our nuclear fuel resources; there is no need for quantities of fertile material larger than those already available as U-238 in our natural uranium resources. The fissile isotope U-233 produced from thorium; however, does have somewhat different characteristics from the fissile plutonium produced from U-238, which result in superior performance when utilized in thermal reactors. The superior performance in thermal reactors of the $11-233$ bred from thorium, as compared to the characteristics of plutonium bred from uranium, provides the incentive for use of thorium fueling.

In particular, the number of excess neutrons available from fission $(n-1)$ of U-233 is larger than that of Pu-239 or U-235 in thermal-spectrum reactors: Representative values of $n$ are given for a typical PUR thermal spectrum in Table 2.1, and illustrates a greater neutron production per neutron absorbed in U-233, particularly with respect to Pu-239. Because of a larger number of excess neutrons froin U-233, more neutrons are available to breed additional quantities of fissile material. When recycle is performed, these

TABLE 2.1

REPRESENTATIVE ETA ( $\eta$ ) VALUES FOR

(Thermal Spectra (Ref. 4))

$\begin{array}{lrrrr} & \text { U-233 } & \text { U-235 } & \text { Pu-239 } & \text { Pu-241 } \\ n(0.025 \mathrm{eV}) & 2.30 & 2.07 & 2.11 & 2.15 \\ n \text { (Typical PI:R Thermal Spectrum) } & 2.27 & 2.06 & 1.84 & 2.17 \\ \begin{array}{l}n \text { epi (Typical PI:R Epi therma1 } \\ \text { Spectrum) }\end{array} & 2.16 & 1.67 & 1.88 & 2.49\end{array}$


differences mean that a sinaller amount of uranium is needed to produce a given amount of energy if thorium is used as the fertile material (i.e., the thorium cycle) than if $U-238$ is used as the fertile material (i.e., the uranium cycle).

Although the $n$ values of U-233 are higher than those of Pu-239 in the thermal spectra characteristic of converter reactors, a different situation exists in the spectra characteristic of liquid metal or gas-cooled fast breeder reactors. Representative $n$ values for the fast breeder reactor are given in Table 2.2. As this table illustrates, the $n$ value of Pu-239 is somewhat higher than that of U-233 in a fast spectrum and that the $n$ value of Pu-241 is significantly higher than that of U-235, the corresponding isotope in the thorium isotopic build-up sequence. Consequently, it can be concluded that $U-233$ is a preferred fissile fuel for thermal spectrum, reactors, but that superior performance is obtained in fast breeder reactors using plutonium fueling.

Within the context of thermal reactors, the $n$ value of $U-233$ is al so much less sensitive to neutron spectrum than that of plutonium which exhibits a

TABLE 2.2

REPRESENTATIVE ETA ( $n$ ) VALUES FOR

Fast Breeder Spectra (Reference 1).

$\begin{array}{ll}\text { Nuclide } & n \\ U-233 & 2.31 \\ \text { U-235 } & 1.93 \\ \text { Pu-239 } & 2.49 \\ \text { Pu-241 } & 2.72 \\ \text { Th-232 } & 0.076 \\ U-238 & 0.411 \\ \text { Pu-240 } & 1.315\end{array}$


decrease ir the number of neutrons produced per fission in a hard (higher energy) thermal spectrum. This characteristic is illustrated in Table 2.3. This attribute of $U-233$ is important in maintaining the nuclear performance high temperature concepts, such as the HTGR.

Another nuclear attribute which is worth noting is that thorium has a higher absorption cross section than does U-238. As Table 2.4 illustrates, the thermal capture cross section of Th-232 is about three times larger than the value of $U-238$, while the Th-232 infinitely dilute resonance integral is about three times smaller than the $U-238$ value. In thermal reactors, lumping of the fuel into rods reduces the effective resonance capture through self-shielding of the resonances, and results in effective resonance capture integrals which are roughly comparable in Th-232 and U-238. The effect of the larger thermal cross section of Th-232; however, leads to the need for higher initial fissile enrichments for thorium cycles.

Overal1, the nuclear properties of U-238 provide an advartage to the uranium cycle, primarily by reducing the fissile requirements for criticality in heterogeneous thermal reactors. The superior performance of U-233 on the other hand, provides an advantage to thorium fueling primarily by increasing the conversion ratio of thermal reactors and

TABLE 2.3

TYPICAL THERMAL-SPECTRUH-AVERAGED ETA VALUES

(Reference 1)

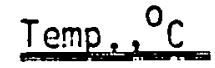

0

300

600

900

\begin{tabular}{llc} 
& Eta & \\
\hline & & \\
U-233 & U-235 & Pu-239 \\
2.29 & 2.06 & 1.87 \\
2.29 & 2.05 & 1.82 \\
2.29 & 2.04 & 1.79 \\
2.28 & 2.03 & 1.77
\end{tabular}


TABLE 2.4

CROSS SECTIONS FOR U-238 and Th-232

(Reference 4)

$\begin{array}{lcccrr} & \begin{array}{c}\text { Thermal } \\ (0.025 \mathrm{eV})\end{array} & \begin{array}{r}\text { Infinitely Dilute } \\ \text { Resonance }\end{array} & \text { Integral } \\ \text { Th-232 } & \underline{U}-238 & & \text { Th-232 } & \text { U-238 } \\ \text { Absorption, } \sigma_{a} & 7.40 & 2.73 & 85.78 & 273.57 \\ \text { Capture, } \sigma_{c} & 7.40 & 2.73 & 85.20 & 272.37 \\ \text { Fission, of } & 0 & 0 & 0.58 & 1.20\end{array}$

hence reducing the quantities of fissile material required to supplement that bred during operation. This competition between the higher initial loadings required with thorium refueling and the reduced annual fissile makeup requirement is discussed in greater detail in Section 2.6.

\subsubsection{Fuel cycles}

basic fuel cycle alternatives are shown in Figure 2.2 for the uranium cycle and in Figure 2.3 for the thorium cycles. Figure 2.2 (a) illustrates the fuel cycle currently in place today where spent fuel is stored either for eventual reprocessing or permanent disposal. Figure 2.2 (b) illustrates the case where uranium and plutonium recycle are performed; the recycle of uranium only could also be contemplated with the plutanium either stored for future use or diverted to the waste stream. Figure 2.2 (c) illustrates a similar recycle mode in which plutonium is utilized in reactors dedicated for that purpose.

A basic thorium fuel cycle is illustrated in Figure 2.3 (a). The initial fissile loading and makeup fissile requirements are provided by highlyenriched ( $93 \mathrm{w} / 0 \mathrm{U}-235$ ) uranium. The $U-235$ remaining in the fuel at end of cycle and the bred U-233 can be recycled as illustrated, while alternately the spent fuel can be stored for future reprocessing or ultimate 
3.2 (a) Once Thungh cuple

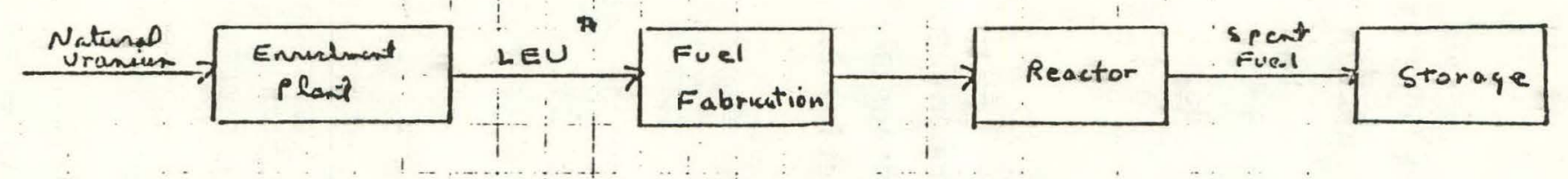

3. $2(b) U$ and Pu Rearie

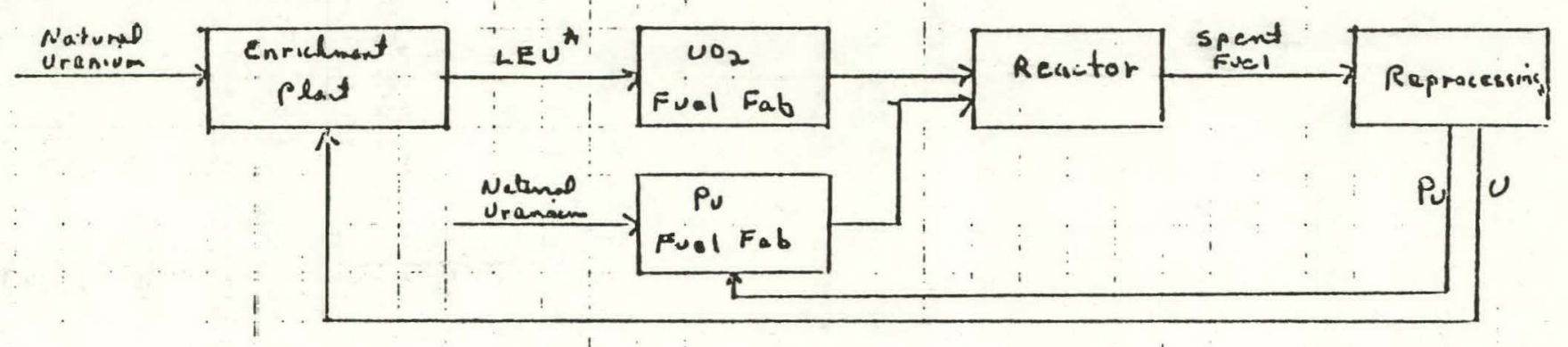

$\stackrel{⿱ ⺊}{a}$

3. 2 (c) Pu Raceple AT Enargy Centers:

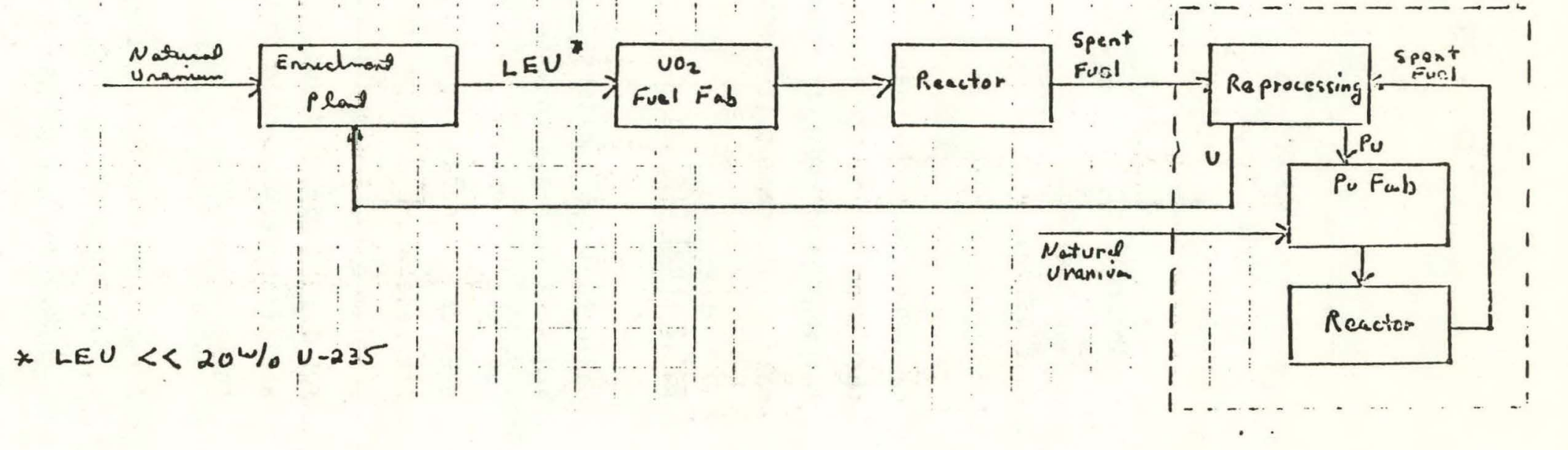


Figure 2.3

Simplified Thorium Fuel Cycles

3.3 (a) Highly Exine d cycle

National

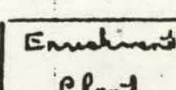

NEv $\sqrt{\text { Fresh Fuel }}$

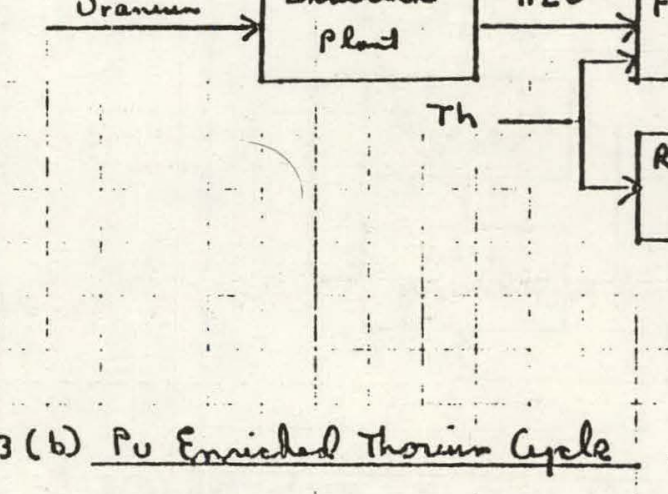

Notarial

Fabrication

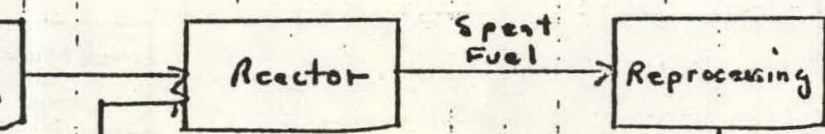

3. 3 (b) Pu Enniaded Thorium Cycle

uranus
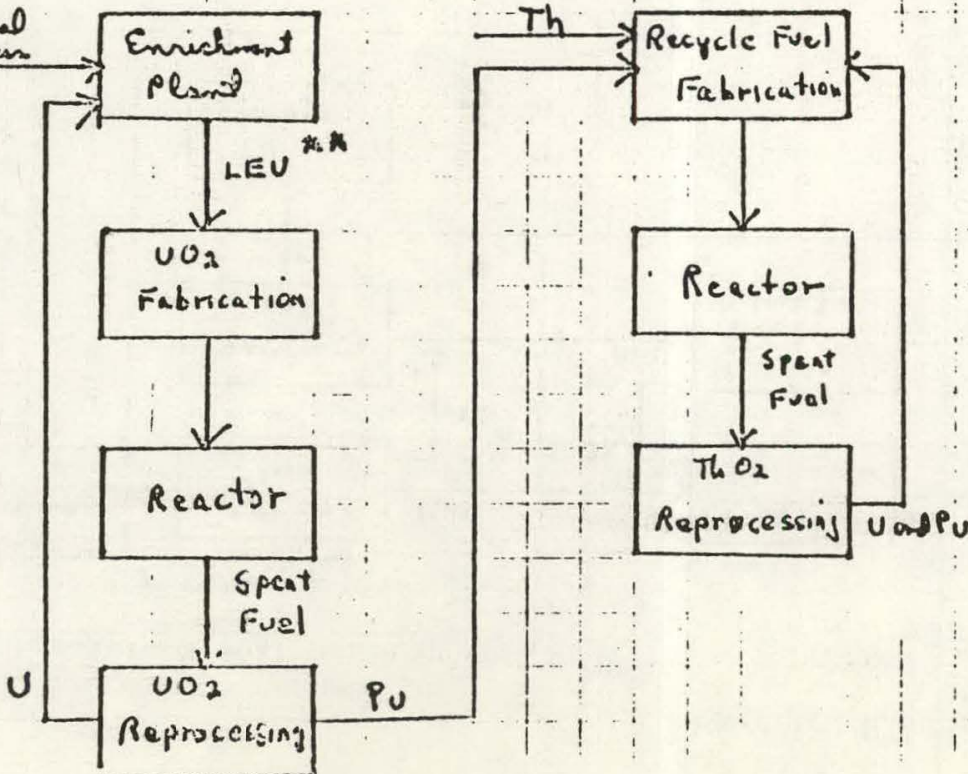

$\stackrel{\stackrel{N}{v}}{*}$ 
$3.3(c)$ Denatured Fuel aycle

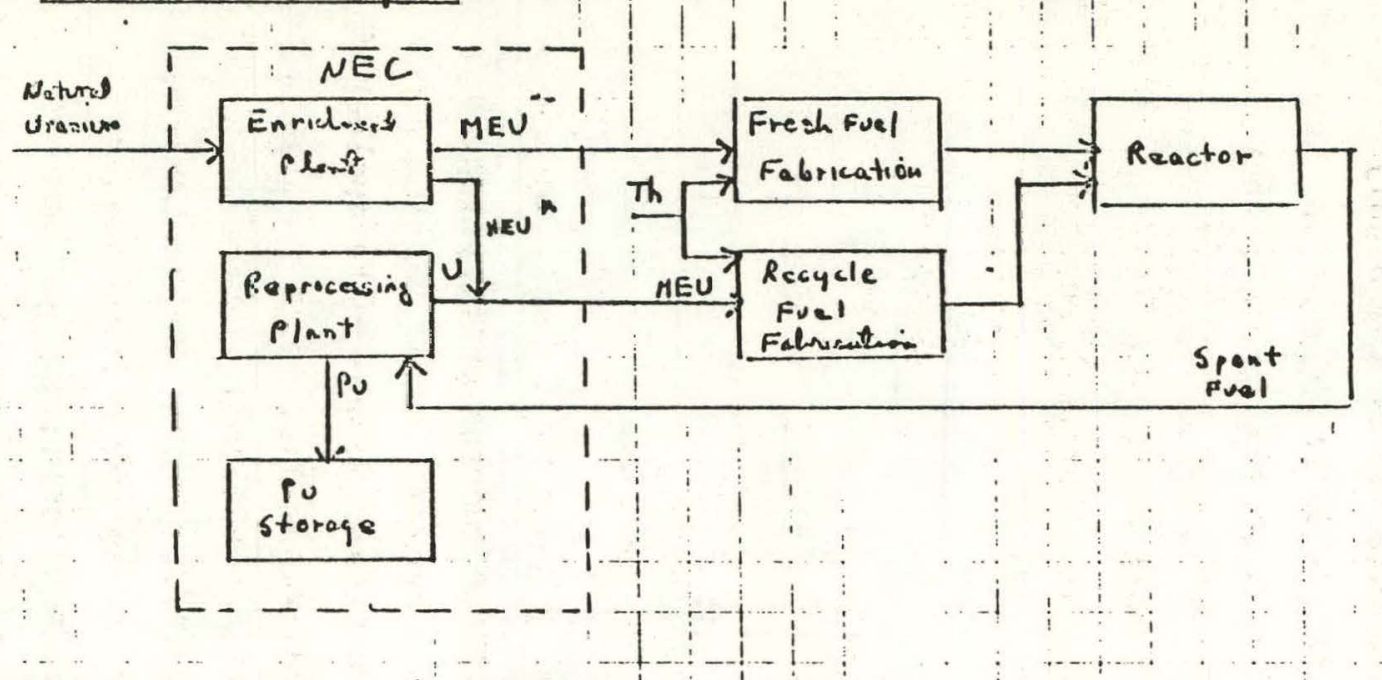

3.3 (d) Dematured Fuel Cycle with F6R

$-x \mathrm{HEU}>20 \mathrm{w} / \mathrm{U}-235 \mathrm{a}>12 \mathrm{w} / \mathrm{U}-233$

$x$ LEV « 20\% V-235 or \&C $12 w / 0 U-233$

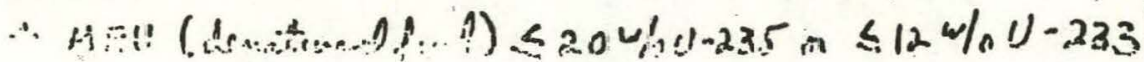


waste disposal. Figure 2.3 (b) illustrates the thorium fuel cycle in which plutonium is used in place of U-235 for enrichnent. This fuel cycle, of course, requires the operation of reactors on the uranium fuel cycle to pro-duce the required quantities of plutonium. Figure 2.3 (c) illustrates the "denatured" thorium fuel cycle. This fuel cycle is similar to the highly enriched case illustrated in Figure 2.3 (a) with the exception that uranium enriched to less than $20 \mathrm{w} / 0 \mathrm{U}-235$ or less than $12 \mathrm{w} / 0 \mathrm{U}-233$ is employed for enrichment rather than the $93 \mathrm{w} / \mathrm{o}$ U-235 material of the highlyenriched thorium fuel cycle. Because of the U-238 content of the denatured fuel, some plutonium will be produced. This plutonium could either be stored as a future natural energy asset as illustrated in the figure; or utilized directly to fuel thorium-based converter reactors (similar to that illustrated in Figure $2.3(b)$ ), or to fuel thorium-bianketed fast breeder reactors as indicated in Figure 2.3 (d).

\subsubsection{Mon-Proliferation Considerations ${ }^{2}$}

The objective of this report is to assess the technical and economic feasibility of thorium utilization rather than to discuss in detail proliferation aspects of the various alternative fuel cycles. Nonet'eless, it is necessary to give some qualitative consideration to non-proliferation aspects in order to establish potential incentives for thorium utilization, and to include in the technical options under consideration those thoriuni refueling alternatives which might contribute to the safeguarding of the nuclear fuel cycle. In revievirig non-proliferation considerations, it is important to recognize that no fuel cycle alternative can completely eliminate the possibility of proliferation. Rather, the objective of proliferation-resistant fuel cycles is to increase the difficulty and length of time required to obtain nuclear weapons materials, so that developing nuclear weapons through power reactor programs becomes unattractive in comparison to other potential technologies. In particular, the objective is to identify fuel cycles which would allow the resource conservation possible with recycle, and eventually with the breeder reactor, to be obtained without increasing the potential for nuclear weapons proliferation. 
U-233, like U-235 or plutonium, can be used to make nuclear weapons. The critical masses of fully reflected spheres of various fissile materials are given in Table 2.5. These.critical masses are indicative of the quantities of fissile material required to construct a nuclear weapon. As the table illustrates, roughly the same quantity of $U-233$ is required as Pu-239. Since the spontaneous fission rate of power reactor grade U-233 is significantly lower than power reactor plutonium, U-233 is probably a more useful material for rapid assembly into prompt critical configurations.

\section{TABLE 2.5}

CRITICAL MASS OF FISSILE SPHERES

REFLECTED BY NATURAL URANIUM ${ }^{2}$

$\begin{array}{lc}\text { Major Isotope } & \begin{array}{c}\text { Critical Mass } \\ (\mathrm{kg})\end{array} \\ \text { U-233 }(98 \%) & 5.63 \\ \text { U-235 }(93 \%) & 17.86 \\ \text { Pu-239 (95\%) } & 5.91\end{array}$

Since U-235 and U-233 can be chemically separated from thorium, just as plutonium can be chemically separated from uranium, the thorium cycles illustrated in figure 2.3 (a) provide as simple a source for diversion of weapons material as the plutonium cycle itself; indeed, greater quantities of weapons-grade material are present in the fuel cycle because of the use of highly-enriched uranium for initial inventories. The plutonium used in the fuel cycle illustrated in Figure 2.3 (b), can also be chemically separated, and it presents a situation analogous to the use of plutonium in uranium fuels.

It should be noted that of primary concern is the presence of weapons-grade material either in pure form or in fresh unirradiated fuel. Weapons material in a pure form, such as may be present at a reprocessing plant, could most easily be utilized in a nuclear weapon; however, a high degree of safeguards can be obtained by applying adequate accountability and physical security 
sasures at the reprocessing facility. The concepts of international and multinational reprocessing centers are currently the subject of investigation, and it appears likely that an acceptable degree of safeguards can be applied to reprocessing facilities within the context of these concepts. Less amenable to physical security measures is the presence of weapons-grade material in fresh unirradiated fuel, since such fuel would have to be shipped to diverse reactor sites. Of less concern is the fissile material contained in spent highly irradiated reactor fuel. Although this material can. be chem-. ically separated, the highly radioactive fission products. necessitate that these chemical operations be performed in elaborate and specially designed facilities. This contrasts to fresh fuel where reprocessing operations would be much simpler because of the absence of the radioactive fission products.

One point worth noting is that recycled $U-233$, although suitable for use in weapons, contains trace quantities of $\mathrm{U}-232$, which is produced during ir radiation in the reactor and which cannot be chemically separated from the fissile U-233. U-232 has daughter nuclides that emit high energy gamma rays. Although the irradiations from $U-232$ would in general be insufficient to preclude assembling a nuclear device, the U-232 contamination does lead to the following non-proliferation advantages:

a. As a result of the gamma ray emissions from the daughters of $U-232$, the sensitivity of special nuclear material detection instruments is significantly greater for U-233 than for plutonium U-239 or uranium U-235. For example, comercially available special nuclear material monitors, which can detect 0.5 gns of $\mathrm{Pu}-239$, should be able to detect less than 0.05 gms of $U-233$ fuel that has not been chemically cleaned for a period of one month or longer.

b. One daughter of U-232, T1-208, emits a 2.6 Hev gamma ray. This high energy gamma has high penetrability in all shielding materials because the minimum mass absorption coefficients for all shields occur near this energy. As a consequence, search and recovery operations for U-233 materials can be assisted by aerial scanning unit: similar to those used for environmental monitoring. 
c. For bulk quantities of U-233, the gamma ray emissions are of an intensity that requires moderate ( 2 to 3 feet of concrete) shielding for processing operations. Thus, access to $\mathrm{U}-233$ is necessarily restricted throughout processing. The dose rates anticipated without shielding are high by occupational exposure standards; however, they are well below dose rates that would cause near-term health effects.

Overall, Th/U-233 fuels are thought to be significantly better than Th/U-235 or plutonium-bearing fuels, but much worse than the low-enrichment uranium fuel currently employed in providing inherent domestic and international safeguards against a possible terrorist explosive threat. This advantage results from the increased detectability of U-233 and from the limited access to $U-233$ necessitated by the shielding required for processing. From the standpoint of international proliferation, U-233-bearing fuels are oniy marginally better than plutonium-bearing fuels because the processing technologies are well within the reach of most nations.

The denatured thorium fuel cycles, illustrated in Figure 2.3 ( $c$ and $d$ ), offer a more significant barrier against nuclear material diversion and weapons proliferation. Such denatured thorium fuel employs uranium enriched to either less than $20 \mathrm{w} / 0 \mathrm{U}-235$ or less than $12 \mathrm{w} / 0 \mathrm{U}-233$ to provide the required fissile materials. Although the uranium can be chemically separated from the thorium, the resulting U-235 or U-233 content is sufficiently small to make construction of a nuclear device impractical. Sophisticated isotopic concentration facilities would be required to concentrate the U-233 or U-235 sufficiently for use in a nuclear weapon. This contrasts with the use of plutoniun or highly-enriched uranium which can be more easily separated from the uranium or thorium diluent using chemical techniques. Because of the mass difference between U-233 and U-238 and the enrichments used in denatured fuels, the concentration of fissile material would be easier (requires less SLU) than U-235 enrichment of natural or slightly enriched uranium. However, although fewer enrichment stages would be required to upgrade denatured U-233 to enrichments suitable for use in nuclear weapons, isotopic separation equipment would still be required, and the development of such equiprient is at 
least currently beyond the capabilities of most lesser developed nations; at any rate, if such isotopic separation equipment were deployed, weapons material could be obtained by the enrichment of natural uranium so that the presence of denatured fuel in the fuel cycle might not necessarily contribute materially to the possibility of obtaining weapons materials.

Overall, the use of denatured fuels might eliminate both domestically and internationaliy the possibility of terrorist groups constructing'a nuclear device. From the standpoint of international proliferation, the use of denatured fuels would impose a significant delay in proliferation because of the necessity of constructing enrichment facilities to concentrate the U-233, or of constructing reprocessing facilities to extract plutonium from the spent fuel. Except for the smaller enrichment capacities needed to enrich $U-233$ fuel, as mentioned above, the effort required to obtain weapons-grade material is equivalent to that which would be required using the low-enrichment cycle currently employed. Consequently, this fuel is judged as significantly better for use in unsecured power reactor plants than either the plutonium-uranium cycle or the highly-enriched thorium cycle, assuming that processing is controlled and performed in safeguarded facilities.

\subsubsection{Thoriun: Supply and Derand}

Thorium is about three times more abundant in the earth's crust than . uranium; reflecting the difference in the half lives of Th-232 $(1.4 \mathrm{x}$ $\left.10^{10} \mathrm{yrs}\right)$ and $U-238\left(4.5 \times 10^{9} \mathrm{yrs}\right)$. However, the recoverable resources of thorium are highly uncertain, because, unlike uranium, the demand and exploration for thorium has been very limited. Total U.S. thorium consumption, in all forms, in 1974 was estimated at $80 \mathrm{ST}^{3}$. Only small.quanties of this thorium were consumed for energy purposes. Except where thorium can be produced as a by-product of uranium mining, the principal source of thorfum is monazite, a by-product of beach-sand mining. Monazite is recovered for its rare earth content; after these rare earths are recovered, the residues may be processed for thorium. In addition to the monazite beach sands, deposits in sedimentary rocks, conglomerates or quartz. sites enriched in thorium and uranium may be thorium sources. Table 2.6 presents 
TABLE 2.6

ASSESSMENT OF H'ORLD THORIUM RESOURCES 3

(Thousand Short Tons)

$\begin{array}{lll} & \text { Other } & \text { Total } \\ \text { Reserves } & \text { Resources } & \text { Resources }\end{array}$

North America:

United States

Canada

$\frac{230}{370}$

$\frac{440}{600}$

$\frac{670}{970}$

South America:

Brazil

\begin{tabular}{lrr}
60 & 100 & 160 \\
10 & 20 \\
\hline 70 & 120 & 30 \\
\hline 190
\end{tabular}

Africa:

Nigeria

Republic of South Africa

15

20

35

other

Total

5

15

20

$\frac{20}{40}$

$\frac{25}{60}$

$\frac{45}{100}$

Europe: USSR

40

80

120

Asia:

India

200

250

450

Other

Total $\frac{20}{220}$

$\frac{30}{280}$

$\frac{50}{500}$

Oceania: Australia

\begin{tabular}{|c|c|c|c|}
\hline & 40 & 100 & 140 \\
\hline World Total & 780 & 1,240 & 2,020 \\
\hline
\end{tabular}


an assessment of known world thorium resources. Although these resources are significant, they are only a fraction of those identified for uranium. However, the discovery of additional thorium deposits would seem likely, should the demand for thorium increase; based on the relative abundance of thorium to uranium, an assumption that thorium resources are at least as large, the projected uranium ore resources seem reasonable.

The requirements for thorium, on the other hand, are only a small fraction of the requirements for uranium ore. As was noted in Section 2.4.1, thorium is an alternative fertile material for U-238. A large fraction of uranium ore is mined to obtain the fissile component $U-235$ which constitutes only about $0.7 \%$ of the uranium. Less than $20 \%$ of the mined ore is actually used as 5 lightly-enriched uranium in reactors, with the remaining $80 \%$ going into diffusion plant tails. Of the $20 \%$ placed into reactors, only a small fraction is consumed during operation; if uranium recycle is performed, the majority of the discharged uranium ends up as diffusion plant tails when the fuel is reenriched. Since thorium is mined strictly as a replacement for the fertile U-238, the demand for thorium would be less than about $20 \%$ of the total uranium ore demand, if discharged thorium were not recycled and even if all. reactors were to employ thoriunil fueling. Recycle of thorium would further reduce derlands to about 5 to $7 \%$ of the uranium denland for a reactor lifetime of 40 years. The considerably lover demand for thorium, together with a relatively large abundance, leads to the conclusion that the size of the thorium resource should not be a limiting factor in the use of the thorium fuel cycle for any reasonable projection of installed nuclear capacity.

\subsection{PEACTCR CIAPPACTERISTICS}

Reviews of thorium utilization indicated that thorium fuel can be employed in most reactor concepts currently deployed commercially or under development. Thus, a variety of reactor applications have been considered in the Thorium Assessment Program (TAP) to evaluate and intercompare the relative merits of thorium utilization in these alternate systems. These reactor systems are summarized briefly below with particular emphasis placed on those aspects which are important to the timely introduction of thorium 
utilization. The discussion of reactor types is followed in Section 2.6 by a discussion of the fuel cycle characteristics. The reactor and fuel cycle characteristics were utilized in systems analysis calculations to determine the overall economics and fuel utilization of nuclear economies containing various mixes of reactor types and fuel cycles. These system analyses, as well as a more detailed ciscussion of the reactor parameters employed, are discussed in Annex 1.

\subsubsection{Light Hater Reactor (LU:R)}

Conventional LWRS - Light-water-moderated reactors of both the bo11ing (BWR) and pressurized water (PWR) types are utilized extensively in the U.S. and throughout much of the world. These reactors have almust universally been fueled with slightly-enriched uranium fuel, although thorium fuel was initially used in the Indian Point Plant of Consolidated Edison. Since the fuel cycle and economic characteristics of the BWR and PWR are very similar in principle, the PWR has been used exclusively in this report as representative of the light-water reactor.

The Conbustion Engineering System $80^{T M}$ reactor design and opcrating characteristics were employed in the analysis of alternate fuel cycles in unmodified LWRs, since evaluations of thorium utilization had previously been performed in this reactor desiyn ${ }^{4}$. The $3800 \mathrm{Mw}(\mathrm{th}), 1300 \mathrm{Mw}(\mathrm{e})$ system 80 T.1 PWR is representative of the large capacity ieactors currently under construction. The reactor design and operating characteristics employed are summarized in Tahle $2 . \overline{7}$.

Characteiristic of Ll:Rs, a regular array of square fuel assemblies is employed within a pressure vessel containing water which serves both as moderator and coolant. For the majority of the cases evaluated, the reference reactor and fuel design as described in this table was unaltered so as to determine the characteristics of thorium utilization in PURs currently in operation or. under construction. The results of the prcliminary evaluation of thorium utilization in the System 80 reactor confirm the suitability.of this reactor design for thorium fueling. In considering methods to improve 
Table 2.7

SYSTEM $80^{\mathrm{TM}}$ CORE PARAMETERS

Core Power, Wivt

Nominal Electrical Output, twe 1300

3800

Fuel Asscmblies

241

Fuel Rods Per Assembly

236

Control Rod Channels Per Assembly

4

Instrumentation Channels Per Assembly

1

Active Fucl Length (inn.)

150

Specific Power $\left(w / \mathrm{cm}^{3}\right)$

94.9

Average Linear Heat Rate $(\mathrm{kw} / \mathrm{ft})$

5.34

\section{OPERATING PARTIETERS}

Primary System Pressure, psia 2250

Reactor Coolant Average T'emperature $\left({ }^{\circ} \mathrm{F}\right) \quad 592$

Nominal Fuel Temperature $\left({ }^{\circ} \mathrm{F}\right) \quad 1500$

FUEL ASSEI:BLIES (Dimensions at $68^{\circ} \mathrm{F}$ )

$\begin{array}{ll}\text { Fuel Rod Array (in.) } & 16 \times 16 \\ \text { Assembly Pitch (in.) } & 8.18 \\ \text { Fuel Cell Pitch (in.) } & 0.5063 \\ \text { Fuel Pellet Diameter (in.) } & 0.325 \\ \text { Zr Clad Thickness (in.) } & 0.025 \\ \text { Gap Thickness (in.) } & 0.00350 \\ \text { Water Channel Between Assemblies } & \\ \quad \text { (half width) (in.) } & 0.0394 \\ \text { CEA/Instrumentation Channel zr Guide Tube: } & \\ \quad \text { ID/OD (in.) } & 0.901 / 0.972 \\ \text { Coolant to Fuel Pellet Volume Ratio } & 1.714 \\ \quad \text { Fuel Cell Ratio } & 2.041 \\ \text { Assembly Ratio }\end{array}$

FUEL LOADINGS

Full $\mathrm{UO}^{\circ}$ Core ('lem)

102.78

Full ThO 2 Core (TeM)

93.50

Fuel steck Density (yin/ $\left.\mathrm{cm}^{3}\right)$

10.05 .1

UO, Fuel

ThO $_{2}$ Fuel

3.173 
fuel utilization within the PUR concept, parameter studies were also performed by varying the basic lattice dimensions or operating characteristics given in the table. These parametric studies illustrated the general rule that annual uranium requirements can be reduced but at the expense of higher initial fissile loadings; alternately, initial loadings can be reduced by lattice modifications but at the expense of higher annual requirements. The thirty-year average uranium requirements prove largely insensitive to lattice design; however, in the longer term, optimized designs can reduce total $\mathrm{U}_{3} \mathrm{O}_{8}$ demand for a given installed nuclear capacity.

The primary incentive for evaluating thorium utilization in LWKS is the potential for rapid and widespread deployment of thorium fuel. The LWR is the only reactor type currently commercially available in the U.S., and essentially all of the nearly $50 \mathrm{Gw}(e)$ of nuclear capacity currently operating in the U.S. are reactors of this type. In view of construction lead times and the period required to commercialize al ternate concepts, reactors of this type will dominate the nuclear economy for at least the next several decades. An LWR base of about $125 \mathrm{Gw}(\mathrm{e})$ is projected for 1985 and the great majority of the additional approximately $200 \mathrm{Gw}(\mathrm{e})$ canacity operating or planned worldwide will be LWRs. Thus, if the thorium fuel cycle is to have a significant impact within the century, it must be implemented in light water reactors.

Spectrum-Shift-Controlled Reactor - The spectrum-sinift-controlled reactor consists of a conventional FWR, as described above, in which the soluble poison control system is replaced with spectrum-shift control to adjust reactivity during normal reactor operation. Spectrum-shift control employs a mixture of heavy and light water in the primary coolant. The introduction of heavy water shifts the neutron spectrum to higher energies and enhances the absorption of neutrons in fertile material; consequently, excess neutrons are utilized to produce additional quantities of fissile material rather than being wasted by absorption in control poisons. Enhanced fuel utilization is achieved by eliminating losses to parasitic captures in control materials in a fashion similar to the improvements in resource utilization achieved with on-line refueling in the CANDU concept or by the relative movement of fertile and fissile fuel regions in the LWBR. 
The spectrunl-shift-contrcl concept hás been demonstrated by the Vulcain reactor experiment in the BR-3 nuclear power plant at Mol, Gelgium. 5 The BR-3 power plant, after two years of operation as a conventional PUR was modified for spectrum-shift operation and successfully operated with this mode of control between 1966 and 1968. The Vulcain core operated to a core average burnup of 23,000 Mwd/T (a peak burnup of around 50,000 $\mathrm{kwd} / \mathrm{T}$ ) and achieved an average load factor and primary plant availability factor of 91.2 and $98.6 \%$ s respectively. The leakage rate of primary water from the high pressure reactor systems to the atmosphere was found to be negligible, about $30 \mathrm{~kg}$ of $\mathrm{D}_{2} \mathrm{O}_{2} \mathrm{H}_{2} \mathrm{O}$ mixture per year. 5

Since the spectrum-shift-controlled reactor is based upon existing lightwater-reactor technology, with modifications required to realize spectrum shift control limited largely to modifications to auxiliary systems, the SSCR has the advantage of potentially early introduction; as demonstrated by the Vulcain experiment, there is also the possibility of backfitting spectrum-shift control to existing PWRs. Another and potentially more attractive alternative is the possibility of modifying plants that are in the eirly phases of construction; this alternative would avoid the potentially costly down-time required to modify operating reactors for spectral shift control. The risk of implementing this concept is also reduced since the reactor could always be operated in the poison-control node. The BR -3 reactor, after completing the Vulcain experiment, was subsequently returned to poison control. Because the financial risk of implementing this concept is limited to the additional cost required to realize spectrun-shift control, a more rapid introduction rate could be contemplated than for a coinpletely new reactor systim; in the latter case, the entire cost of the reactor and the generating capacity of the plant are at risk. The primary technical difficulties with realizing. this concept are the heavy water supply and problems with the control of primary coolant leakage and refueling.

Other Light-Water-Reactor Concepts - Two other light-water-reactor concepts. al though not discussed in detail in this report, should be mentioned." The " first of these is the light-water-breeder reactor (LWBR) concept developed 
at Bettis. A demonstration of this concept is scheduled to begin in late 1977 at the Shippingport reactor.

The design of the LWBR is made unusual by the need to avoid parasitic neutron losses. In the Shippingport demonstration, the necessary reactivity adjustment to compensate for fission product buildup is provided by an arrangement for moving highly reproducing fuel within a blanket region. In addition, the spacing of fuel rods is much closer than in conventional LWR to attain high conversion.

The LWBR is fueled with thorium enriched with U-233. A conversion or a breeding ratio 1 to $2 \%$ in excess of unity is anticipated with the thorium fuel cycle, which, in principle, would allow unlimited continued operation without addition of fissile material once the initial inventory is provided. With these fuel cycle characteristics, the fuel demand becomes closely related to the installed nuclear capacity rather than to annual energy output. The fuel for new breeder reactors is assumed to be produced entirely from normal LWRs, with fuel assenblies especially designed to perform efficient thoriuni to U-233 conversion. Thus the system is composed of two separate components-the pre-breeder to produce the initial inventories and the hold-own breeder itself.

The primary attraction of the LUBR concept is the very high long-term uranium ore utilization possible within the context of the well-developed light-water reactor technology. Utilization of existing LURs as prebreeders appears feasible and, as the Shippingport project is to demonstrate, it is possible to convert existing LW:P.S to LWBR operation. ATthough conversion to LUBR operation appears possible, there remains a question of whether such conversion can be achieved without seriously derating the power output of the reactor and hence compromising the power cost econonics. A second consideration is the high fissile inventory required for such operation. Seven to fourteen years of pre-breeder operation are required to produce the U-233 inventories necessary to start up the LUIBR. 
TABLE 2.8

\section{CAMDU-PHIIR CORE PAPAMETERS}

Core Power, Mut.

Moninal Electrical Output, Mue

Thermal Efficiency, \%

Number of Fuel Channels

Specific Power, kw/kg

Average Linear Heat Rate, kw/ft

Discharge Exposure (Hat $\mathrm{UO}_{2}$ ), Mwd/TeM-

Fuel Management

Operating Parameters

Primary System Pressure, psia

Reactor Coolant Average. Temperature, ${ }^{\circ} \mathrm{F}$

Fuel Assemblies

Geometry

Orientation

Active Length, in.

Bundle Diameter, in.

Number of Fuel Rods/Bundle

Fuel Rod Diameter, in.

Fuel Pellet Diameter, in.

Fuel Pellet Density, $\mathrm{g} / \mathrm{cm}^{3}$.

Fuel Rod Clad.

\section{Fuel Loading}

Fuel Loading/Bundle, kgu

18.3

Number of Fuel Assenblies
2510

750

29.8

480

22

6.69

8000

Continuous Refuel ing
1420

542

Cylindrical

Horizontal.

19.5

4.03

37

0.516

0.479 .

10.55

$\mathrm{Zr}$ 
Consequently, the LIABR seens more suited to periods when low electrical growth is anticipated.

In the seed-and-blanket reactor, fissile material is bred in the blanket from neutrons which leak from the enriched seed material. As fissile material is bred in the blanket, power generation in the blanket increases as the fissile material is consumed in situ. At the end of its useful reactivity lifetime, the enriched seed material is replaced with fresh fuel (the seed can be placed into, the blanket region to achieve a more complete burnup of the contained material), while the blanket material remains in core for a number of cycles in order to achieve high burnup and to more efficiently utilize in situ its bred fissile content.

The primary problem with realizing economic performance in the seed and blanket concept is the imbalance in power which can result between the low enriched blanket region and the highly enriched seed naterial. Because of this imbalance in power, the average power densities achieved are usually significantly lower than those in conventional LURs; as a result, capital costs are increased. Additional evaluations of this concept are required in order to establish its resource utilization characteristics and the anticipated fuel and power costs. Since the resource requirements for. the LWBR are very closely approximated by those of the CANDU reactor operating on a self-sufficient cycle, the LIVBR concept was not included explicitly in the systems analysis performed for this study; very similar resource utilization results would have been obtained had the LWBR been substituted for the self-sutficient CANDU reactor.

\subsubsection{Heavy liater Reactors (HisR)}

The CAINOU-PHIIR (Pressurized Heavy later Reactor) served as the reference HWR concept for the thorium assessment program. Typical core parameters for the CANDU PHWR are given in Table 2.8. A number of reactors of this type are already in operation in Canada and have been exported to a number of other nations. CANDU PHWR are considered to be the only reactor type 
besides the LWR for which a significant commercial manufacturing base presently exists; the British and French gas-cooled reactors have not gained acceptance outside their countries of origin.

The PHIIR is characterized as having cylindrical fuel assemblies located in pressure tubes through which flows heavy-water coolant. The pressure tubes are arranged in a widely-spaced array in the calandria (tank) containing heavy-water moderator. CANDU plants employ on-line refueling and current designs use natural uranium fuel, although operation with slightly-enriched uraniun or thorium fuels is also possible. 7

Two CAIIDU reactors were considered in the TAP evaluations: the first was a $600 \mathrm{M}$ (e) Gentilly II plant currently under construction in Quebec; the second was a conceptual design for a "Middletown," U.S.A. plant which was siliilar to Gentilly II, but increased in size to $3800 \mathrm{Nw}(\mathrm{th})(1140$ Mw(e)) and modified as much as necessary to meet U.S. licensing criteria.

The primery advantage of the CANDU PHWR is the improved resource utilization which results from the better neutron economy possible with heavy water and on-line fueling. On the thorium cycle, self-sufficient breeding (i.e., no uranium ore required in the equilibrium cycle) is possible, although discharge burnups are relatively low; economic considerations may favor higher burnups for which supplementary uranium ore is required. The highly successful operation of the CANDU reactors deployed in Canada offers the potential for the rapid introduction in other areas of the world, including the U.S.

The main difficulty with introducing CANOU into the U.S. is the lack of domestic manufacturing experience and questions of design modifications which might be dictated by U.S. licensing criteria and traditions. A large 3800 liw(th) plant, upon which the economic analysis contained in this report is predicated, would aiso have to be developed and demonstrated. U.S. heavy water production capacity would also have to be increased if a significant rate of CANDU introduction were to be supported.

There are also a number of heavy-water reactor concepts in addition to the PHWR. The Gentilly I plant empinys light. water as a reastonr conolanti while 
retaining heavy-water moderation (CANDU-BLR) and utilizes natural uranium fueling. The steam-generating heavy-water reactor (SGHWR), exemplified by the prototype operating at Winfrith in Great Britain, is a similar concept which utilizes low-enrichment uranium fuel. In general, the resource utilization of these light-water-cooled reactors are intermediate between that of the PHWR and the LWR; the main incentive for this reactor type is the potential for reduced capital cost resulting from decreased reactor size and heavy-water inventory. Another meniber of the CANDU family of reactors is the organic-cooled heavy-water-moderated reactor (CNiNU-OCR). The primary motivations for this reactor are the potential reduction in capital cost and the higher efficiency possible with organic coolant. Although a small demonstration reactor using organic coolant is in operation in Canada, significant additional research, development and demonstration will probably be required before this concept can be considered for widespread deployment. Heavy-water reactors of the pressure vessel type have also been constructed, for example the Atucha plant in Argentina, and resource utilization comparable to the CANOU PHIS have been obtained by employing on-line refueling in this plant.

\subsubsection{High Temperature Gas-Cooled Reactor (HTGR)}

The reference HTGR is based upon the conceptual design of a $1344 \mathrm{Mw}(\mathrm{e})$ commercial plant developed by $G A^{8,9}$. Specifications for this plant are given in Table 2.9. The HTGR employs coated fuel particles contained in high integrity graphite fuel elements (prisms) through which the hel ium coolant flows. The reference HTGR has a nuclear conversion ratio of about 0.67 and provides a steam cycle with an efficiency of $40 \%$. The higher efficiency compared to the $33 \%$ of the LWR, is the major reason that the reference HTGR achieves better uranium utilization than the LWR per unit of electrical energy output. Modifications, such as an increase in the thorium-to-carbon ratio, semi-annual refueling, reduced power density, and reduced discharge burnup, can also be made to increase the conversion ratio, and hence further improve resource utilization ${ }^{10}$ at some expense to capacity factor, reprocessing and capital costs. 
TABLE 2.9

\title{
HTGR CORE PARAMETERS
}

Core Power, Mwt.

Nominal Electrical Output, Mwe

1344

Thermal Efficiency, \%

40

Core Power Density, W/CC

7.1

Fuel Assembly Geometry.

Hexagonal Blocks

Primary Pressure, psia

775

$C / U$ or $C / T h$ Ratio

Discharge Burnup, Mwd/MTHM

$\therefore 150-650^{*}$

$47,000-196,000 *$

\author{
*Depends on Fuel Cycle \\ *Depends on Fuel Cycle. See Annex 1, Table C.2.
}

U.S. experience with high-temperature gas-cooled reactors dates to March 3, 1966 when the 40 Mw(e) Peach Bottom Atomic Power Station became operable. More recently, the $330 \mathrm{Mw}(e)$ Fort St. Vrain Station has been completed and is currentiy undergoing low-power test operation. Extensive experience with gas-cooled reactors has also been obtained in Great Britain where 31 plants having a total capacity of $7738 \mathrm{Mw}(\mathrm{e})$ are in operation.

Since thorium fueling has always heen the reference fuel cycle for the HTGR, more extensive development of the HTGR thorium fuel cycle has been performed than in other reactor concept. The HTGR also takes advantage of the nuclear performance of U-233. (relative to $(1-235$ or Pu-239) to obtain relatively high conversion ratios while operating at the elevated tenperatures which result in increased thermal efficiency. Because of the HTGR. fuel design, which consists of microspheres containing fissile and fertile fuel materials contained in fuel channels within graphite fuel elenents, a wide latitude of fissile-to-fertile and heavy-metal-to-moderator atom 
ratios are achievable without alteration of the basic concept of the fuel design. These parameters control, to a large extent, the conversion ratio of the reactor system, and thus the current-design HTGR is amenable to alterations in conversion ratio over a fairly large range. For this reason, the HTGR has the advantage of having a high degree of flexibility to tailor the reactor fuel cycle by varying the fuel loading and fuel exposures to meet changes in economic and resource availability conditions.

Real constraints on HTGR commercialization and deployment have been encountered in both the U.S. and Europe, and it is difficult to summarize the present situation and future expectations in any simple way; however, these difficulties are manifested by the history of attempts to comnercialize the HTGR in the U.S.

\section{5 .4 Licuid Metel Fast Bresder Reactor (LMFBR)}

The reference LMFBR (c.f. Table 2.10) employed in the Thorium Assessment Program is based upon the conceptual design of a large low temperature prototype breeder reactor developed by the Hanford Engineering Development Laboratory 11 . The LMFBR employs a regular array of hexagonal fuel elenents containing fuel rods clad with stainless steel, about which the liquid sodium coolant flows. The central region of the core contains enriched fuel, while the axial and radial bianket regions contain depleted uranium. The PLBR employs oxide fuel, has a specific inventory of about $5000 \mathrm{~kg}$ fissile plutonium per Gw(e), and exnibits a compound fissile doubling time of about 12.5 years when operated with the uranium blanket or about 13.1 years with thorium blankets.

The primary advantage of the LIIFER (or any real breeder concept) is that it produces. a surplus of fissile material which can subsequently be used either to expand nuclear capacity or to supplement uranium requirements of converter reactors. Rather extensive development of the LMFBR concept has been performed both in the U.S. and overseas. LMFBRs are currently in operation in England (PFR - $250 \mathrm{Mw}(e)$ ), in France (Phoenix $233 \mathrm{Nw}(e))$, and in the USSR (350 $\mathrm{MW}(e))$, and a larger $600 \mathrm{MW}(\mathrm{e})$. LMFBR is scheduled to begin operation in 1978 in the USSR. 
TABLE 2.10

LMFBR CORE PARAMETERS

Core Power, Niwt

3736

Nominal Electrical Output, Mwe

1196

Thermal Efficiency, :

Average Linear Heat Rate, $\mathrm{kw} / \mathrm{ft}$

Core/Axial Blanket/Radial Blanket

$8.9 / 0.4 / 1.3$

Reactor Inlet/Outlet Temperature, ${ }^{O_{F}}$

$620 / 900$

Refueling Interval

annual

Fuel Residence Time, FPO

525.6 :

Discharge Exposure, Mwd/kg $\left(\mathrm{UO}_{2}\right)$

61.6

Fuel Assemblies

Number of Assemblies

380

Number of Fuel Rods/Assembly

271

Assembly Length, in.

120.9

Lattice Pitch, in.

6.22 .

Duct Wall Thickness, in:

0.084

Interduct Gap, in.

0.303

Pellet Diameter, in.

0.2565

Pellet Density, $\mathrm{gm} / \mathrm{cc}$

10.23

Cladding 00 ; in.

0.286

Cladding Thickness

0.012

Cladding Matertal

316 SS, $20 \% \mathrm{cW}$

Diametral Gap, in. .0055

Beyond the normal technical development problems inherent in such a large undertaking, new difficulties have emerged, particularly in the U.S. for the LMFBR concept relating to the possibility of increased nuclear weapons proliferation potential should the plutonium-fueled LMFBR be deployed widely throughout the world. Intense growth of this concern has resulted in the postponement of U.S. plans to construct a facility which would demonstrate concepts and components necessary for the eventual construction of large 
LMFBRs and the subsequent comnercial deployment of this type of plant. In order to appraise the possibilities for reducing proliferation concerns associated with LMFBRs, a number of thorium-fueled cores have been included in the thorium assessment program to estabiish the characteristics and potential of these alternate fuel cycles.

\subsubsection{Gas-Cooled Breeder. Reactor (GCFR)}

vesos

The gas-cooled breeder reactor consists of a four-enrichment zone core of $\mathrm{PuO}_{2}$ and depleted uranium. surrounded by an axial blanket of depleted uranium and a radial blanket comprised of either uranium oxide or thorium . oxide. The GCFR is basically a blend of HTGR and LMFBR technologies: the fuel element design, technology developed for the LMFBR and the gas-coolant technology of the HTGR would be utilized in this concept, so as to minimize the required research and development effort. Evaluations of the GCFR have indicated that the fuel utilization performance of this reactor is comparable to the LiMFBR ${ }^{12}$. Consequently, both the LMFBR and the GCFR are represented in the thorium assessment study. as a single category, the fast breeder reactor (FBR).

\subsection{FUEL UTILIZATIOH}

\subsubsection{Fuel Cycle Options}

Assessments are currently under way by ER.DA and other agencies of the Federal Government to establish non-proliferation criteria against which various fuel cycle options can be evaluated. When these criteria are developed, they will provide important guidance to the selection of fuel cycles optimal from the standpoint of non-proliferation. In the Thorium Assessment Program, no attempt was made to judge these criteria by eliminating from consideration fuel cycle alternatives. However, in order to allow the fuel cycle alternatives developed in the Thorium Assessment Program to be employed in non-proliferation assessments, care has been taken to include alternatives which might have inherent non-proliferation advantages, and the cases evaluated were divided into fuel cycle 
options which could be readily distinguished in terms of the degree of resistance to proliferation. These fuel cycle options are summarized below.

\section{Once Through (Throwaway) Cycle}

The Once-Through Cycle in which spent fuel is stored rather than reprocessed is the fuel cycle currently in place today. Reactor options with this fuel cycle which were considered inclided the standard PIIR; modifications of the standard PWR, including Both minor modifications and the implementation of more major changes," such "as spectrum shift control or the seed and blanket concept; the standard CANDU utilizing both natural uranium and slightly enriched uranium fueling, the HTGR utilizing both low-enrichment uranium and denatured thorium fueling; and the use of the tandem PWR-HWR cycle. In the latter; spent PWR fuel is reinserted into a heavy-water reactor for an additional cycle of irradiation: 13 .

2. U-233 and U-235 Recycle

This fuel cycle option allows only the recycle of uranium. Included are the highly-enriched thoriun cycles in the. PUP, FBR, HTGR and CAHDU reactors, in addition to the conventional uraniun cycle with uranium recycle.

3. Pu-U Recycle lith Restricted Pu Utilization

In this fuel cycle option, $\mathrm{Pu}-U$ recycle is restricted to reactors located at secure energy centers. All reactors located outside of these secured areas (dispersed reactors) are restricted to the use of denatured fuels unsuitable for use in nuclear weapons: either low-eririchment uranium as currently employed, or denatured thorium. Denatured thorium consists of a mixture of thorium and uranium in which the uranium component is enriched to less than $20 \mathrm{w} / 0 \mathrm{U}-235$ or less than $12 \mathrm{w} / 0 \mathrm{U}-233$. Plutonium produced by dispersed reactors 
TNUE 2.11

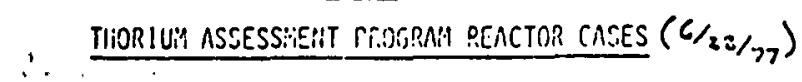

ONCE-THROUGH (THROHAWIAY) CYCLE

PHR-US (LE)/U

PWR-US (DE)/U/TH PWR-US (HE)+IJ3(RE)/TH

$P H R-U S(D E) / U / T_{H}$

PHR-U3 (HE)/TH

$P H R-P U / U$

PHR $-P U / T_{H}$

PHR-US (LE)/U-EE

PWR-US(LE)/U-TAI

Ph'R-US(LE)/U-SS

P'AR-US (HE) +U3 (RE)/TH-SS

P'́R-US (DE)/U/TH-L'HB(SB)

PUR-US(DE)/U/TH-HAB (SB)

FRR-PU/U/U/U

FBR-PU/U/TH/TH

FBR-U3/U/TH/TH

FRR-II3/TH/TH/TH

HTGR-US(DF)/U/TH-T

HTGR-US (ḦE)/TH

HTGR-LS(LE)/U

HTGR-U3 (HE)/TH

HIGR-US (Ut)/U/IH

HTGR-U3 (DE)/U/TH

CANDU-U5 (SEU)/U

CALOU-US (NAT)/U

CRIIDU-US (DIS)/U-TAN

CANLU-US (DE)/U/TH-T

CAHDU-US (HE)/TH

CANDU-U3 (HE) $/ T_{H}$

$x$

$-$
PU-U RECYCLE RESTRICTED TO

SECURE. CEHTERS(C) AHD DIS-

PERSED(D) REACTORS RESTRICTED

TO "DENATL'AED" PENCTORS

FULL RECYC:
RECYCLE

$x$

$-$

$x$

$-$

$-$

$-$

$\bar{x}$

D
D
-
D
-
-
C
-
-
-
-
-

$x$

-

-

$x$

-

-

-

-

$x$

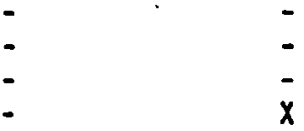

-
D
-

$-$

$-x$

$x \quad x$

-
$\bar{x}$
-
-
-
-
-
$x$ $\dot{x}$

$\bar{x}$

$x$

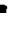

$-$ 
is utilized at the secured centers to fuel converter or breeder reactors which contain thorium in the blanket and/or core regions. $\mathrm{U}-233$ produced by center reactors is denatured with $\mathrm{U}-238$ and can then be employed in dispersed reactors. Also included in this option is the use of the highly-enriched thorium cycle at the secured centers. Reactors considered for deployment at energy centers consist of the PUIR, the FBR, the HTGR and the CANOU reactor, while PWRs, FBRs and CANDU reactors operating on denatured fuel. cycles were considered

for dispersed-site use.

4. Full Recycle

The standard full recycle option was included" in the thorium assessment program as a reference case against which the-advantages and disadvantages of other alternatives can be compared. This fuel cjcle, of course, has the advantage that reactors are currently operating with uranium fueling and producing the plutonium required for recycle in standard LIRs or to initiate the deployment of plutonium-fueled LMFBRs. Included in this option are the reference cases for most reactor types, i.e., the PUR with uranium and plutonium recycle, the FBR operating on the plutonium cycle, and the HTGR operating on the highly-enriched thoriun cycle with recycle of uranium.

\subsubsection{Fuel Cycle Characteristics}

A comprehensive characterization of fuel utilization requires detailed consideration of the cycle-by-cycle fuel requirements for each alternative reactor concept and fuel cycle. Such detalled material flows were employed in the economics and resource evaluation.* In this section, a simplified characterization has been employed to summarize the fuel utilization of varlous alternatives, in which initial loading requirements, the cquilibrium cycle uranium demand and the 30-year uranium requirements have been employed to characterize the performance of converter reactors.

* See HEDI, Reactor Design Characteristics and Fuel Inventory Data (September 1977). 
This simplified characterization is useful since it does delineate life-time uranium requirements and provides a concise basis for intercomparing alternatives; however, it must be recognized that the time-dependent (cycle-by-cycle) requirements are important in economic assessments and in the evaluation of annual uranium demands during periods of nuclear expansion.

For all cases discussed below, $\mathrm{U}_{3} \mathrm{O}_{8}$ requirenents are given for a $1000 \mathrm{llw}$ (e) reactor having an assumed 1 ifetime of 30 years. A $75 \%$ capacity factor and a 0.2 w/o diffusion plant tails assay were also assumed, along with a twoyear out-of-reactor time for reprocessing and refabrication. The worth of fuel remaining in the reactor or in reprocessing and refabrication at the end of the assumed 30-year lifetime has been neglected. For breeder reactors, fuel utilization is tabulated in terms of the specific inventory and system doubling times, since these are more appropriate measures of system performance. Initial inventories have also been translated into the equivalent $U_{3} O_{8}$ requireitents, based upon the uranium ore consumption of light-water reactors required to produce initial fissile loadings.

\section{Light-l!ater Keactors}

$\mathrm{U}_{3} \mathrm{O}_{8}$ requirements for the reference LUR design are summarized in Table 2.12. The requirements shown for reactor start-up include both the initial core and the first two reloads, i.e., total uranium utilized before recycle begins in the third reload core. Two cases are tabulated for the denatured thorium cycle. In the first case, only uranium recycie is performed, with the recovered plutonium stored for eventual use in the breeder reactor: The second option utilizes both uranium and plutonium recycle. Here plutonium is recycled into thorium fuel in plutonium enriched converter reactors of the reference system 80 design. These reactors would be located at secured energy centers and the U-233 which is produced is assumed to be denatured and returned to reactors located at dispersed sites. As Table 2.12 illustrates, the effect of thorium utilization is to reduce annual 
TABLE 2,12

$U_{3} \mathrm{O}_{8}$ REQU.IREMENTS FOR LIIRS (ST $\mathrm{U}_{3} \mathrm{O}_{8} /$ GWe) *

Reactor Annual 30-Year

Startup Requirement Reguirement

Uranium Cycle

Once Through

791

$\sigma_{\text {G }}$, wo:

$U$ Recycle.

$U$ and Pu Recycle

791

$\therefore b=192$

6000

791

154

4950

4100

Thorium Cycle

Highly-Enriched $U$ Recycle

1104

87

3450

Denatured U Recycle

1189

110

4100

Deriatured $U^{2}$ and Pú Recycle

1189

.94

3750

\section{*75\% Capacity Factor, 0.2 w/o Tails Assay}

$\mathrm{U}_{3} \mathrm{O}_{8}$ requirements to about $58 \%$ of that obtained on the once-through uranium cycle.

For all cases shown in Table 2.12, the reference PWR parameters have been utilized and the discharge burnup has been assumed to be 30,400 Miwd/T for $\mathrm{UO}_{2}$ fuels and $33,400 \mathrm{Mwd} / \mathrm{T}$ for $\mathrm{ThO}_{2}$ fuels."

Figure 2.4 presents the cumulative $\mathrm{U}_{3} \mathrm{O}_{8}$ ore requirements as a function of years of reactor cis tion for the once-through uranium cycle, the

*The difference in burnup results from the difference in density of UO? and $\mathrm{ThO}_{2}$; the burnups are selected so that the number of full power days between refuelings remiains constant. 


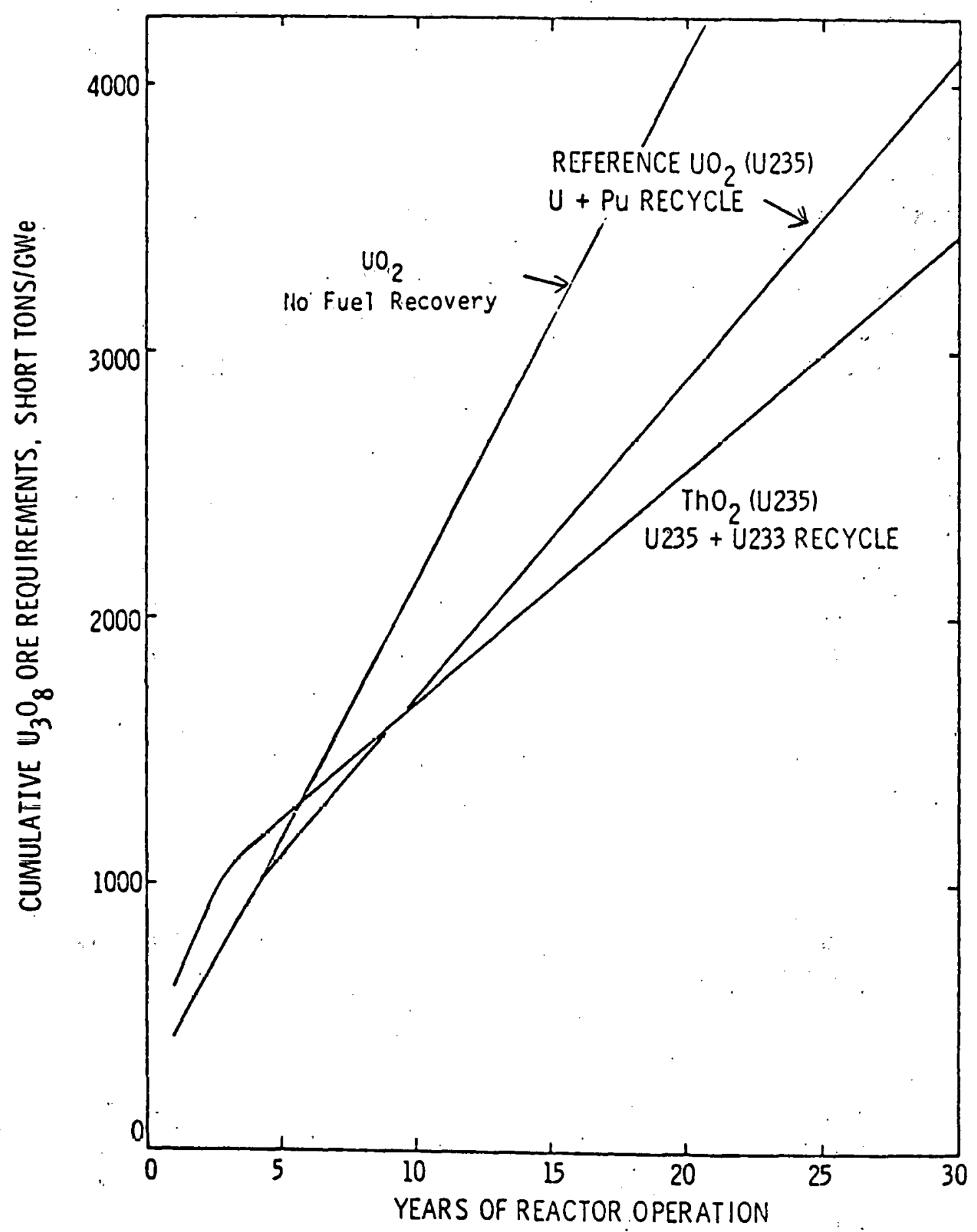

Figure 2.4 CUMULATIVE $U_{3} \mathrm{O}_{8}$ ORE REQUIREMENTS VS REACTOR LIFETIME 
uranium cycle with uranium and plutonium recycle, and the highly enriched thorium fuel cycle options. As this figure illustrates, reactor startup requirements are higher for the thorium cycle as compared to the uranium cycle. This results from the higher absorption cross section of thoriun as compared to U-238. However, because of the higher corversion ratio possible in thoriumfueled systems, the annual ore requirements are lower than those of the uranium cases and within 6 to 10 years of operation (depending on whether the thoriuln cycle is compared to the throw-away uranium cycle or to the uranium cycle with $U$ and $\mathrm{Pu}$ recycle), cumulative $\mathrm{U}_{3} \mathrm{O}_{8}$ ore consumption is lower with thorium fueling. Figure 2.5 illustrates cumulative separative work requirements as a function of years of reactor operation. Initial separative work requirements are higher with thorium fueling than for uranium fueling because of the higher initial loadings of thorium cores and the use of highlyenriched uranium. Over the reactor's 30-year lifetime, separative work consumption is higher than if uranium and plutonium recycle were Derformed, but roughly equivalent to the demand of the throw-away cycle. Although these curves of cumulative $\mathrm{U}_{3} \mathrm{O}_{2}$ and Sl:U requirements were generated for the light-water reactor, the trends illustrated here of higher initial uranium and Sl:U requirements and of lower annual $\mathrm{U}_{3} \mathrm{O}_{8}$ demand are also characteristic of the inter-comparison of thorium and uranium cycles in other reactor types.

For all cases shown in Table 2.12 and in Figures $: 2.4$ and 2.5 , the reference PWR parameters have been utilized and the discharge burnup has been assumed to be 30,400 Mwd/T (33,400 Mwd/T for ThO2 fuel). Modifications of such fuel cycle parameters as discharge burnup can result in some improvement in resource utilization. Improvements such as this, which can be implemented without modifying reactor design, were included in the thorium assessment evaluation and are described in Annex 1. Further improvements in resource utilization could, of course, he achieved in the L!h by lattice optimization; however, these alterations to the Lin design would typically compromise the advantage of early implementation of the thorium cycle 


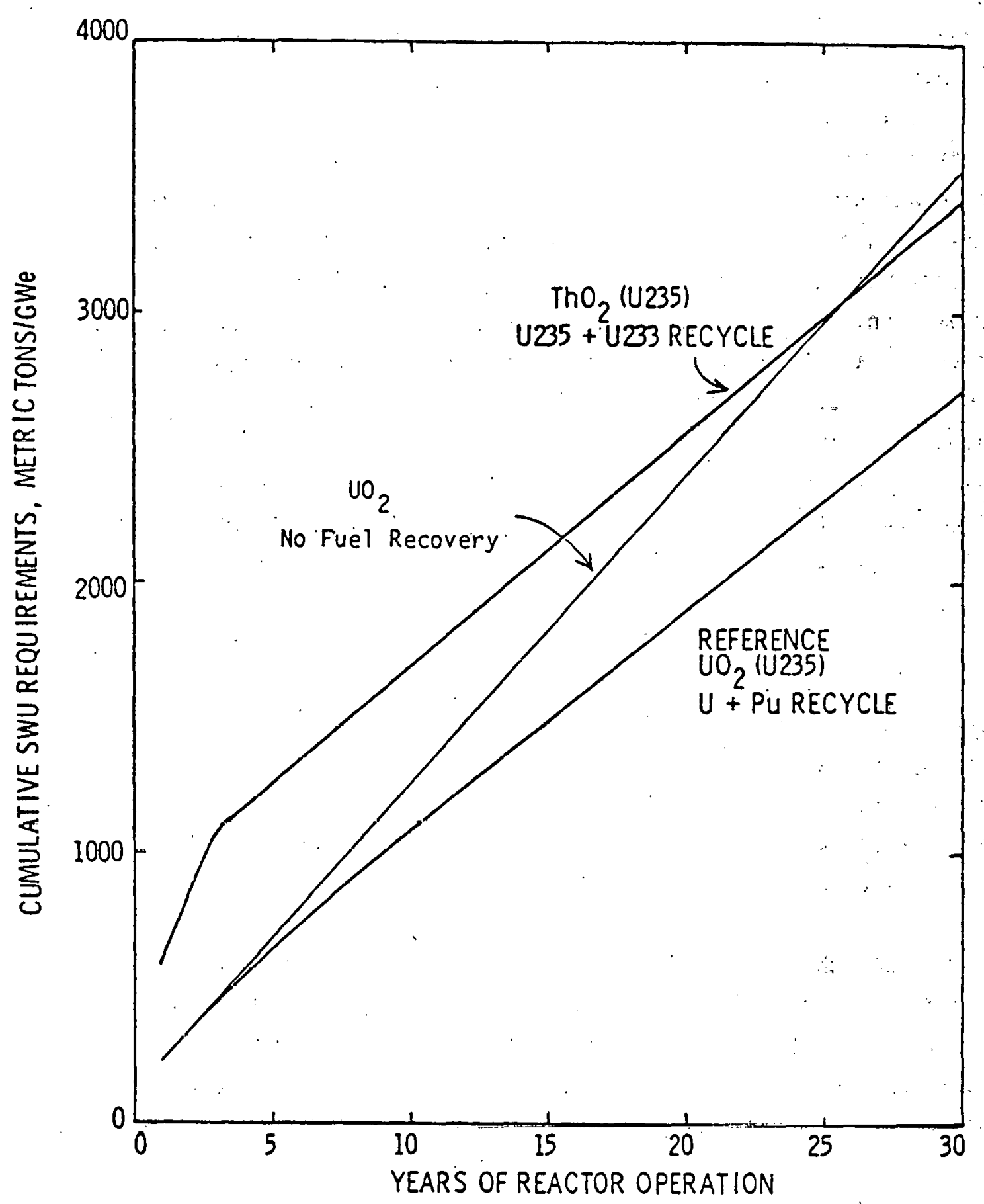

Figure 2.5 CUMULATIVE SWU REQUIREMENTS VS REACTOR LIFETIME 
and/or the economics of thorium utilization. Consequently, the fuel utilization parameters employed in the thorium assessment program were based upon the use of thorium fueling in a conventional PIIP design without modification. In general, modifications could be utilized which would reduce the higher initial loading requirements of the thorium fuel cycle, but at the expense of a somewhat reduced conversion ratio and hence of higher annual $\mathrm{U}_{3} \mathrm{O}_{8}$ requirements; alternately, the conversion ratio could be improved somewhat, reducing annual $\mathrm{U}_{3} \mathrm{O}_{8}$ demand, but at the expense of higher initial loadings. This trade-off between initial $\mathrm{U}_{3} \mathrm{O}_{8}$ requirements and annual $\mathrm{U}_{3} \mathrm{O}_{8}$ requirements is a general characteristic of lattice optimization in any reactor system. The optimal design from a resource utilization standpoint will consequently depend not only upon the detailed reactor material inventory balances, but also upon the assumed rate of nuclear expansion. The establishment of optimal reactor design was beyond the scope of the Thorium Assessment Program.

The resource utilization for the reference pressurized water reactor modified for spectrum shift control is given in Table 2.13. This reactor consists of the standard System 80 PIIR in which the poison control system has been replaced with spectrum-shift control achieved through the introduction of varying concentrations of heavy water. The lattice parameters and discharge burnups are unal tered from the reference uranium cycle case.

On the once-through uranium cycle, startup requirements and 30-year requirements are reduced somewhat from those required with poison control (5000 for the SSCR versus 6000 with poison control). On the thorium cycle requirements are reduced to between 2200 and 2500 ST, depending on the thorium fuel cycle option.

\section{Heavy Water Reactors}

$\mathrm{U}_{3} \mathrm{O}_{8}$ utilization in heavy-water reactors of the CANDU type is summarized in Table 2.14. Natural uranium fueling, the mode of fueling currently employed in CANDU reactors, is shown for reference. Improved 
TABLE 2.13

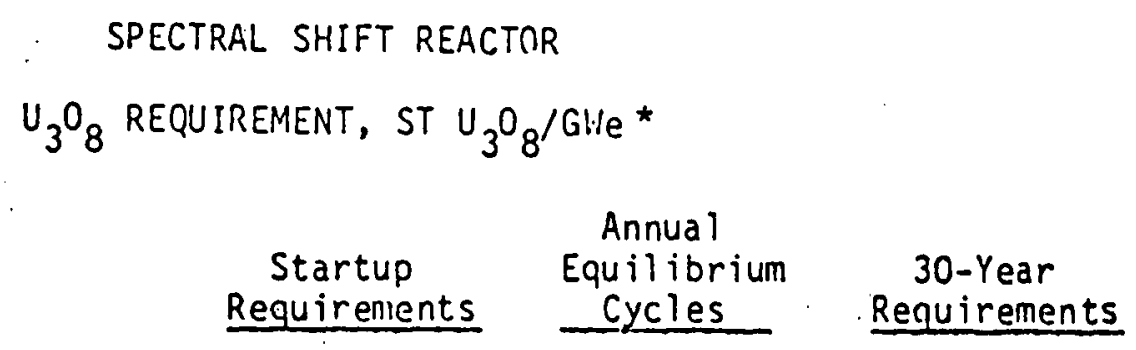

Requirements Cycles Requirements

Uranium Cycle

Once Through

658

161

5010

Thorium Cycle

Highly Enriched, U:Recycle

1029

43

2185

Denatured; U Recycle

1120

48

2500

Denatured, $U+P u$ Recycle

1120

44

2200

*75\% Capacity Factor, 0.2 w/o Tails Assay

resource utilization can be obtained by using slightly enriched uranium fuel, with a concomitant increase in discharge burnup. The case illustrated, which utilizes 1.2 w/O U-235 enrichrient and achieves a burnup of about $20,600 \mathrm{Fild} / \mathrm{T}$ is thought to be near optimal for the CANOU-PHIR, al though only slightly higher uranium requirements result when higher enrichments and discharge exposures are utilized, as illustrated in.-

Figure 2.6.

Also given in Table 2.14 are the $U_{3} U_{8}$ requirements for the highly enriched and denatured thorium fuel cycles. Annual uranium requirements for the HWR are, of course, dependent upon the conversion ratio achieved, and the conversion ratio, in turn, is dependent upon the discharge burnup. As the discharge burnup is decreased, less neutrons are lost to parasitic captures and fission products, resulting in an improved conversion 
TABLE 2.14

$\mathrm{U}_{3} \mathrm{O}_{8}$ REQUIRE!HENTS FOR HIRS (ST $\mathrm{U}_{3} \mathrm{O}_{8} / \mathrm{GWe}$ ) *

\begin{tabular}{|c|c|c|}
\hline $\begin{array}{l}\text { or } \\
\text { up }\end{array}$ & $\begin{array}{c}\text { Annual } \\
\text { Reguirement }\end{array}$ & $\begin{array}{l}30-\text { Year } \\
\text { Requirement }\end{array}$ \\
\hline
\end{tabular}

Uranium - Once Through

Natura $1 \mathrm{U}$

LEU
504

359

112

3390

Thorium Cycle (Highly Enriched)

$$
\begin{aligned}
\text { Burnup } & =27 \mathrm{Mwd} / \mathrm{kg} \\
& =16 \mathrm{Mwd} / \mathrm{kg} \\
& =8.5 \mathrm{Mwd} / \mathrm{kg}
\end{aligned}
$$

Thorium Cycle (Benatured)
760

902

1470

886
38

27

0

38
1790

1631

$1470^{* *}$

1912

\footnotetext{
* 75\% Capacity Factor, 0.2 Tails Assay

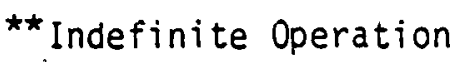

ratio. This dependency of conversion ratio on discharge exposure is, in principle, applicable to other concepts besides the MIIR; however; the on-line fueling employed in the HWR makes this concept uniquely suitable for efficient operation with low discharge burnups without jeopardizing the plant capacity factor. As Table 2.14 illustrates, the 30-year ore requirements are 1770 when a burnup of $27 \mathrm{Mwd} / \mathrm{kg}$ is employed. This burnup is roughily comparable to that of the LWR and hence the amount of fuel which must be reprocessed and refabricated is also comparable. As the burnup is decreased, the conversion ratio increases until at a burnup of between 8.5 and $10 \mathrm{Mwd} / \mathrm{kg}$ a conversion ratio of 1 is achieved. At this burnup a self-sufficient breeder is realized, i.e., there are no annual uranium requirements. Operation 
Fi. 2.0 Fuel Utilization Characteristics for Enriched

Urarium Fueled CANDU (Ref. 14)

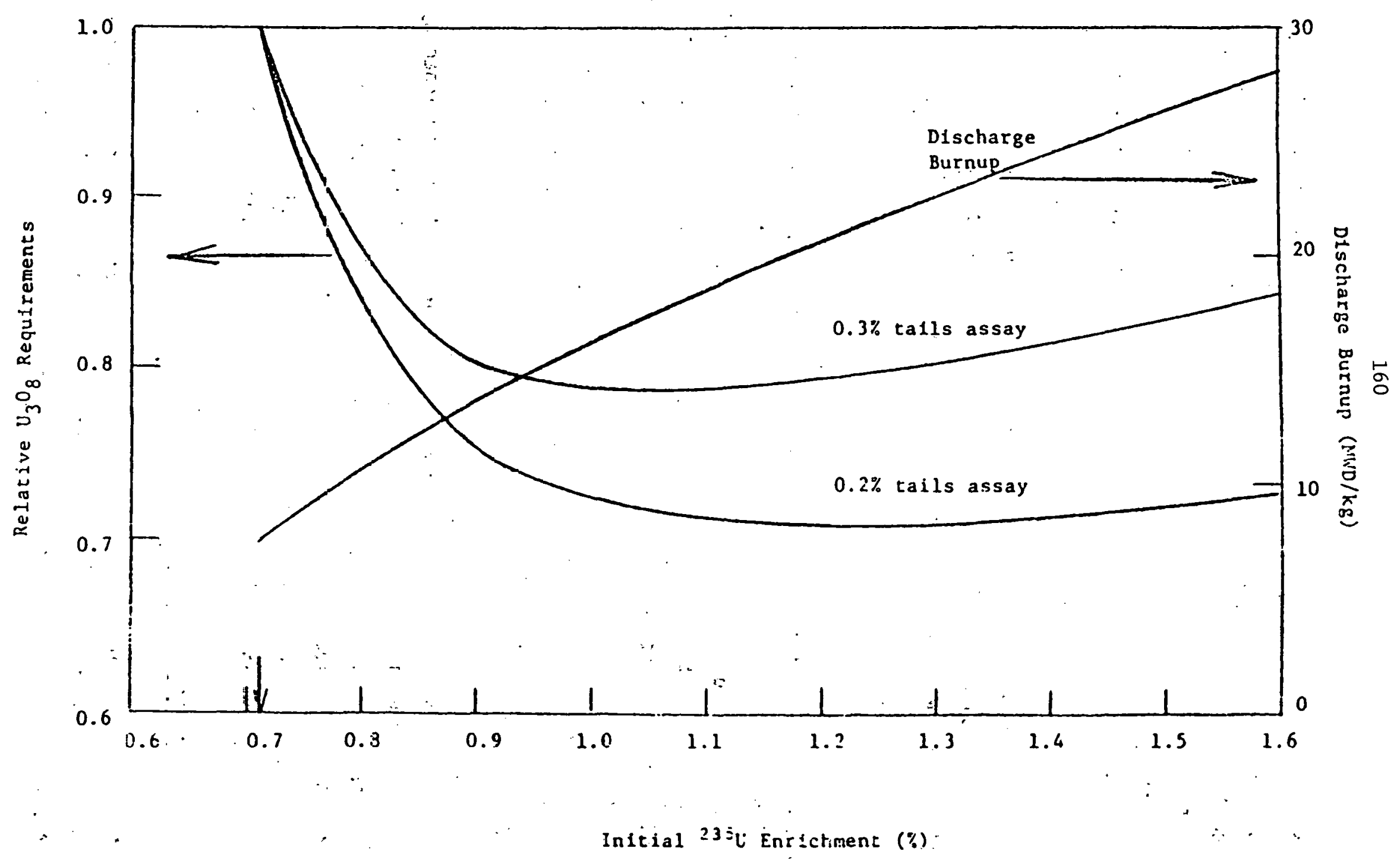


at this burnup, however, requires two to three times the volume of fuel to be reprocessed and refabricated relative to the LUR, with a concomitant increase in she fuel cycle cost for these services. The economic optimum for the HWR is, consequently, a tradeoff between the increased cost for fuel fabrication and reprocessing as the burnup is decreased and the reduction in uranium demand realized with reduced burnup.

As Table 2.14 illustrates, the 30 -year uranium requirements for the denatured thorium fuel cycle in the HWR are roughly comparable to those for the highly-enriched thorium cycle.

\subsubsection{High-Ternerature Gas-C.00led Fenctor}

$\bigcup_{3} O_{8}$ resource utilization is summarized in Table 2.15 for the HighTemperature Gas-Cooled Reactor (HTGP). The once-through cycle, which employs a feed of highly-enriched uranium $(93 \mathrm{w} / 0(1-235)$ in thorium is tabulated for the reference HTGR design, which achieves a conversion ratio of 0.66 ; this design is considered optimum under current economic conditions. $\mathrm{U}_{3} \mathrm{O}_{8}$ resource utilization is al so given for the low-enrichment, once-through uranium cycle and for the denatured thorium fuel cycle with $U$ recycle. Resource utilization is roughly comparable between these three alternatives.

Fuel utilization parameters are also given for the highiy enriched cycles where uranium is recycled, both for the reference design and for a high conversion ratio design. The high conversion ratio design employs an increase in thorium loading, semi-annual refueling, reduced power densities, and some modification to the fuel rod and/or fuel particle design. Conversion ratios intermediate between these two are, of course, possible.

\subsubsection{Breeder Rsactors}

Table 2.16 summarizes the specific inventories and doubling times for breeder reactors. The reference breeder concept is the LMFER containing a $\mathrm{UO}_{2}$ blanket. This reactor is based upon HEDL's low-temperature oxide 
TAELE 2.15

HIGH TEMPERATURE GAS COOLED REACTORS

$\mathrm{U}_{3} \mathrm{O}_{8}$ REQUIREMENTS, ST $\mathrm{U}_{3} \mathrm{O}_{8} /$ Gile *

Annual

$\begin{gathered}\text { Requirements } \\ \text { Requiremear } \\ \text { Cycles }\end{gathered}$
Requirements

Thorium Cycles

Highly Enriched Once Through

512

4300

Highly Enriched, U-Recycle

$C R=0.66$

512

79

2650

$C R=0.90$

.810

28

1575

Denatured, U Recycle

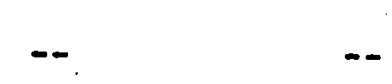

4100

*75\% Capacity Factor, 0.2 w/o Tails Assay

design, and its performance is considered to be characteristic of a number of a ternate LMFBR designs. The introduction of thorium blankets in place of the $\mathrm{UO}_{2}$ blankets presently employed in the reference design has little effect on the compound fissile system doubling time, increasing the doubling time from 12.5 to 13.1 years when both axial and radial uranium blankets are replaced with thorium. However, most of the breeding gain is in the form of U-233 production so that an exogenous source of plutonium is required to expand LMFER capacity. With the use of thorium radial blankets and uranium axial blankets, a Pu fissile doubling time of about 26 years is achieved. The denatured LlifBR design employs the some type of denatured fuel as has been considered in converter reactors, i.e. a mixture of uranium and. thorium with the uraniuri component enriched to $12 \mathrm{w} / \mathrm{O} U-233$. Such breeder reactors could be deployed at dispersed sites without concern that fresh fuel would be diverted and processed into weapons material. Although 
TABLE 2.16

BREEDER REACTOR

Specific Inventory.

Doub? ing Time

$\mathrm{kg}$ fiss/Gue
ST $\mathrm{U}_{3} \mathrm{O}_{8}$

Years

LMFBR

$\mathrm{vO}_{2}$ blanket

4600

1500

12.5

Tho, blanket

4600

1500

26

Denatured

GCFR

$\mathrm{UO}_{2}$ blanket

4600

1500

15.5

$\mathrm{ThO}_{2}$ blanket

4600

1500

ح25 3)
1) Fissile $\mathrm{Pu}$ discarded - $410 \mathrm{~kg} / \mathrm{GW}(\mathrm{e})-\mathrm{yr}$
Net Feed Requirement $=250 \mathrm{~kg} \mathrm{U}-233 / \mathrm{GW}(\mathrm{e})-\mathrm{yr}$
2) Increase in LWR requirements as a result of plutonium diversion to $F B R$; assumes $0.2 \mathrm{w} / 0$ tails assay.

3) Pu fissile doubling time

the denatured LMFBR is a breeder reactor, producing more fissile material each year than consumed, a net feed of $250 \mathrm{~kg} \mathrm{U}-233 / \mathrm{GW}(\mathrm{e})-\mathrm{yr}$ is required. The excess fissile which is produced is in the form of plutonium, with $410 \mathrm{~kg} / \mathrm{Gw}(e)-\mathrm{yr}$ produced. This material would either have to be discarded or used in secured energy centers.

Also given in Table 2.16 are the specific inventory and doubling times for similar $\mathrm{UO}_{2}$ or thorium blanket GCFR designs. As Table 2.16 notes, 
the performance of the GCFR is thought to be comparable with that of the LMFBR.

\subsection{Fuel Cycle Characteristics Utilized in Thorium Assessinent Program}

As indicated in Section 2.6.2, a variety of fuel cycle performance characteristics can be obtained within the context of any one reactor design through reactor design optimization and through the modification of such operating parameters as discharge burnup. Further optimal economic performance "is not necessarily consistent with optimal resource utilization; the optimum design from the standpoint of power cost economics depends on such parameters as the price of uranium ore, separative. work, reprocessing fabrication, and the effective capital costs of each option in addition to the resource utilization performance. In order to redure the number of cases to a manageable number, the thorium assessment program's evaluation was restricted to fuel cycle options considered representative of the various reactor types and fuel cycle alternatives. The lifetime performance characteristics of the reactors and fuel cycles included in the thorium assessment program evaluation is summarized in Table C.2 in Annex 1. 
REFERENCLS:

1. WASH 1097, "The Use of Thorium in Nuclear Power Reactors," June 1969.

2. J. E. Rushton, "A Safeguards Assessment of $U^{233}$ Fuel Utilization (draft).

3. "Thorium," Preprint from Pulletin 667, Mineral Facts and Problenis, 1975 Edition, Bureau of Mines, United States Department of the Interior.

4. N. L. Shapiro, et al., "Assessment of Thorium Fuel Cycles in Pressurized Water Reactors," EPRI HPP-359, Feb. 1977.

5. J. Storrer, "Experience With the Construction and Operation of - the BR3/ VULCAIN Experiment," Symposium on Heavy Water Povier Reactors, IAEA, Vienna, 11-15 September 1967.

6. J. Storrer, et al. "Belgonucleaire and Siemens PIIRs for Small and Medium Power Reactors," Proceedings of a Symposium on Small and lledium Power Reactors, IAEA, Oslo, 12-16 October 1970.

7. J. S. Foster and E. Critoph, "The Status of the Canadian Nuclear Power. Program and Possible Future Strategies," Annals of Nluclear Energy; Vol.2, 1975.

8. P. R. Kasten, et al., "Assessment of the Thorium Fuel Cycle in Power Reactors;" ORNL/TM-5565, Jan. 1977.

9. L. L. Bennett and R. K. Lane, "Fuel Management Flexibilities of Th- ${ }^{233} U$ Cycle," Proceedings of American Nuclear Society Topical Meeting on GasCooled Reactors, Gatlinburg, Tenn., May 1974.

10. R. H. Brogli, "The High Conversion HTGR for Resource Conservation," GA-A13606, October 1975.

11. "Reactor Design Characteristics and Fuel Inventory Data," HEDL, Septenber 1977.

12. P. R. Kasten, et al., "Assessment of the Thorium Fuel Cycle in Powcr Reactors," ORNL/TM-5565, January 1977.

13. A. J. Frankel and N. L. Shapiro, "Appraisal of PVIR-HWR Tandem Fuel Cycles," NPSD-45, Feb. 1977.

14. C. E. Till and Y. I. Chang, "Candu Physics and Fuel Cycle Analys is," Quarterly Progress Report for the Period January-iarch 1977. 
THIS PAGE

\section{WAS INTENTIONALLY LEFT BLANK}


ORNL/TM-6332

Dist. Category UC -80

\section{Internal Distribution}

1. T. D. Anderson

2. S. Baron

3. D. E. Bartine

4. H. F. Bauman

5. H. W. Bertini

6. J. E. Bigelow

7. J. O. Blomeke

8. H. I. Bowers

9. J. T. Bradbury

10. R. A. Bradley

11. R. E. Brooksbank

12. W. D. Burch

13. T. J. Burns

14. W. L. Carter

15. J. C. Cleveland

16. T. E. Cole

17. A. G. Croff

18. J. F. Dearing

19. J. G. Delene

20. J. R. Engel

21. D. E. Ferguson

22. B. C. Finney

23. G. F. Flanagan

24. M. H. Fontana

25. A. J. Frankel

26. E. H. Gift

27. W. R. Grimes

28. W. O. Harms

29. J. F. Harvey

30. D. E. Hatch

31. C. R. Hudson

32. J. D. Jenkins

33. P. R. Kasten

34. W. J. Lackey
35. M. Levenson

36. A. L. Lotts

37. R. L. Lowrie

38. F. C. Maienschein

39. J. D. McGaugh

40. L. E. McNeese

41. F. R. Mynatt

42. K. J. Notz

43. M. J. Ohanian (IEA)

44. A. R. Olsen

45. H. Postma

46. R. T. Primm

47. B. E. Prince

48. W. R. Ragland

49. W. A. Rhoades

50. P. S. Rohwer

51. T. H. Row

52. R. T. Santoro

53. D. L. Selby

54. R. I. Shoup

55. R. L. Simard

56. M. J. Skinner

57. G. R. Smolen

58-107. I. Spiewak

108. W. G. Stockdale

109. D. B. Trauger

110. H. E. Trammell

111. J. E. Vath

112. R. P. Wichner

113. R. G. Wymer

114-115. Central Research Library

116. Document Reference Section

117-118. Laboratory Records Department

119. Laboratory Records, RC

\section{External Distribution}

DOE, Washington, DC 20545

120. W. W. Ballard, Assistant Director, Fuel Cycle Development

121. E. S. Beckjord, Director, Division of Nuclear Power Development

122. S. T. Brewer, Program Planning and Analysis

123. Phil Clark, Associate Director for Reactors, Division of Naval Reactors

124. E. G. Delaney, NASAP Control Office, Office of Fuel Cycle Evaluation

125. R. P. Denise, Assistant Director for Special Projects, Nuclear Regulatory Cómission 
126. A. P. D'Zmura, Division of Nuclear Power Development

127. D. E. Erb, Division of Reactor Research and Technology

128. Herb Feinroth, Office of the Director, Division of Nuclear Power Development

129. Neil Goldenberg, Division of Advanced Systems and Materials Production

130. E. J. Hanrahan, Director, Office of Fuel Cycle Evaluation

131. Hugh Kendrick, Office of Fuel Cycle Evaluation

132. M. W. Koehlinger, Program Planning and Analysis

133. P. M. Lang, Assistant Director, Light Water Reactor Programs

134. K. O. Laughon, Assistant Director, Gas Cooled Reactor Programs

135. D. E. Mathes, Office of Fuel Cycle Evaluation

136. S. E. Miller, Program Planning and Analysis

137. J. A. Patterson, Chief, Supply Evaluation Branch, Division of Uranium Resources and Enrichment

138. W. S. Scheib, Division of Nuclear Fuel Recycle and Production

139. C. Sege, Office of Fuel Cycle Evaluation

140. J. Shibel, office of Fuel Cycle Evaluation

141. Saul Strauch, Office of Fuel Cycle Evaluation

142-166. K. Trickett, Office of the Director, Division of Nuclear Power Development

167. Director, Research and Technical Support Division, DOE, ORO

168. Director, Reactor Division, DOE, ORO

169. H. W. Behrman, DOE, ORO

170. D. Linz, DOE, ORO

171. Robert Avery, Argonne National Laboratory, 9700 South Cass Avenue, Argonne, IL 60439

172. R. M. Ball, Babcock \& Wilcox, P.O. Box 1260, Lynchburg, VA 24505

173. L. L. Bennett, International Atomic Energy Agency, Kartner Ring 11, P.O. Box 590, A-1011, Vienna, Austria

174. Henry Bermanis, United Engineers \& Constructors, Inc., 30 South 17th Street, Philadelphia, PA 19101

175. Y. Chang, Argonne National Laboratory, 9700 South Cass Avenue, Argonne, IL 60439

176. S. Cohen, Teknekron, 4701 Sangamore Road, Washington, DC 20016

177. Michael J. Driscoll, Department of Nuclear Engineering, Massachusetts Institute of Technology, 138 Albany sereet, Cambridge, MA 02139

178. M. C. Edlund, Virginia Polytechnic Institute, Blacksburg, VA 24060

179. J. F. Foran, Resource Planning Associates, Inc., 3 Enbarcadero Center, Suite 2080, San Francisco, CA 94111

180. W. Hardie, Hanford Engineering Development Laboratory, P.O. Box 1970, Richland, WA 99352

181. Robert Hellens, Director, Special projects, Cuibustion lifininecring, Inc., 1000 Prospect Hill Road, windsor, CT 06095

182. Walter C. Lipinski, Argonne National Laboratory, 9700 South Cass Avenue, Argonne, IL 60439

183. M. Mathez, Minerals Advisor, Exxon Corporation, Avenue of the Americas, New York, NY 10020

184-188. R. P. Omberg, Hanford Engineering Development Laboratory, P.O. Box 1970, Richland, WA 99352 
189. C. I. Rickard, Executive Vice President, General Atomic Company, P.0. Box 81608, San Diego, CA 92138

190. Norton I. Shapiro, Manager, Advanced Design Projects, Combustion Engineering, Inc., 1000 Prospect Hill Road, Windsor, CT 06095

191. B. I. Spinrad, Department of Nuclear Engineering, Oregon State University, Corvallis, OR 97331

192. H: B. Stewart, Nuclear Technology Evaluations Company, ,4040 Sorrento Valley Blvd., Suite F, San Diego, CA 92121

193. Tom Sobey, S. M. Stoller Corporation, Suite 815, Colorado Bldg., Boulder, $\mathrm{CO} 80302$

194. Edward Teller, University of California, Lawrence Livermore Laboratory, P.0. Box 803, Livermore, CA 94550

195. C. E. Till, Argonne National Laboratory, 9700 South Cass Avenue, Argonne, IL 60439

196. R. F. Turner, General Atomic Company, P.O. Box 81608, San Diego, $C A \cdot 92138$

197. H. E. Williamson, Manager, Systems Analysis, Nuclear Engineering Operational Planning, General Electric Company, 175 Curtner Avenue San Jose, CA 95125

198. T. D. Willson, Resource Planning Associates, Inc., 44 Brattle Street, Cambridge, MA 02138

199-337. For distribution as shown in TID-4500 under UC-80, General Reactor Technology. 